\title{
UNE VERSION FEUILLETÉE ÉQUIVARIANTE DU THÉORÈME DE TRANSLATION DE BROUWER
}

\author{
par Patrice LE CALVEZ
}

\begin{abstract}
The Brouwer's plane translation theorem asserts that for a fixed point free orientation preserving homeomorphism $f$ of the plane, every point belongs to a Brouwer line: a proper topological embedding $\mathbf{C}$ of $\mathbf{R}$, disjoint from its image and separating $f(\mathrm{C})$ and $f^{-1}(\mathrm{C})$. Suppose that $f$ commutes with the elements of a discrete group $\mathrm{G}$ of orientation preserving homeomorphisms acting freely and properly on the plane. We will construct a G-invariant topological foliation of the plane by Brouwer lines. We apply this result to give simple proofs of previous results about area-preserving homeomorphisms of surfaces and to prove the following theorem: any Hamiltonian homeomorphism of a closed surface of genus $g \geq 1$ has infinitely many contractible periodic points.
\end{abstract}

\section{Définitions et notations}

On écrira $\bar{X}, \operatorname{Int}(\mathrm{X}), \partial \mathrm{X}$, pour l'adhérence, l'intérieur et la frontière respectives d'une partie $\mathrm{X}$ d'un espace topologique $\mathrm{E}$.

Pour tout groupe $G$, on notera $G^{*}$, l'ensemble $G$ privé de son élément neutre.

Le plan euclidien $\mathbf{R}^{2}$ sera muni de son orientation usuelle.

Une surface désignera ici une variété topologique connexe sans bord orientée de dimension deux, non nécessairement compacte.

On appellera disque ouvert (resp. disque fermé) d'une surface $\mathrm{M}$ toute partie homéomorphe à

$$
\mathbf{D}=\left\{z \in \mathbf{R}^{2} \mid\|z\|<1\right\}
$$

(resp. à $\overline{\mathbf{D}})$.

On appellera anneau d'une surface $\mathrm{M}$ toute partie homéomorphe à $\mathbf{T}^{1} \times \mathrm{I}$, où $\mathbf{T}^{1}=\mathbf{R} / \mathbf{Z}$ et $\mathrm{I}$ est un intervalle non réduit à un point. Dans le cas où $\mathrm{I}$ est ouvert (resp. compact), on parlera d'anneau ouvert (resp. d'anneau compact).

On appellera demi-plan fermé de $\mathbf{R}^{2}$ toute image de $[0,+\infty[\times \mathbf{R}$ par un homéomorphisme de $\mathbf{R}^{2}$. On appellera bande fermée de $\mathbf{R}^{2}$ toute image de $[0,1] \times \mathbf{R}$ par un homéomorphisme de $\mathbf{R}^{2}$.

On notera $\mathbf{T}^{2}=\mathbf{R}^{2} / \mathbf{Z}^{2}$ le tore de dimension 2 .

On appellera arc une application continue $\gamma$ d'un intervalle I de $\mathbf{R}$ dans une surface $\mathbf{M}$, ou plus précisément une classe d'équivalence d'applications par reparamétrage préservant l'orientation. On utilisera souvent, par abus de langage, 
le mot arc pour désigner son image, en faisant bien attention qu'il n'y ait pas d'ambiguité. Si $\gamma_{1}: \mathrm{I}_{1} \rightarrow \mathrm{M}$ et $\gamma_{2}: \mathrm{I}_{2} \rightarrow \mathrm{M}$ sont deux arcs tels que

$$
\sup I_{1} \in I_{1}, \quad \inf I_{2} \in I_{2}, \quad \gamma_{1}\left(\sup I_{1}\right)=\gamma_{2}\left(\inf I_{2}\right),
$$

on notera $\gamma_{1} \gamma_{2}$ l'arc naturellement défini par assemblage de $\gamma_{1}$ et $\gamma_{2}$. Si J est un intervalle de $\mathbf{Z}$ et si $\left(\gamma_{j}\right)_{j \in \mathrm{J}}$ est une famille d'arcs $\gamma_{j}: \mathrm{I}_{j} \rightarrow \mathrm{M}$ telle que

$$
\sup \mathrm{I}_{j} \in \mathrm{I}_{j}, \quad \inf \mathrm{I}_{j+1} \in \mathrm{I}_{j+1}, \quad \gamma_{j}\left(\operatorname{sup~} \mathrm{I}_{j}\right)=\gamma_{j+1}\left(\inf \mathrm{I}_{j+1}\right),
$$

si $j$ et $j+1$ appartiennent à $\mathrm{J}$, on écrira $\prod_{j \in \mathrm{J}} \gamma_{j}$ l'arc défini par assemblage des $\gamma_{j}$. Un arc $\gamma: \mathrm{I} \rightarrow \mathrm{M}$ tel que

$$
\sup I \in I, \quad \inf I \in I, \quad \gamma(\sup I)=\gamma(\inf I)=z,
$$

est un lacet basé en z.

On notera $\gamma_{1} \wedge \gamma_{2}$ le nombre d'intersection algébrique de deux arcs définis sur une surface $M$ quand il est bien défini, en particulier quand les arcs sont tous deux des lacets, quand l'un des arcs est propre et que l'autre est un lacet, ou encore quand les deux arcs sont définis sur des intervalles compacts et que les extrémités d'un arc n'appartiennent pas à l'autre arc. Par convention de signe, on écrira $\gamma_{1} \wedge \gamma_{2}=1$, si $\gamma_{1}: \mathbf{R} \rightarrow \mathbf{R}^{2}$ et $\gamma_{2}: \mathbf{R} \rightarrow \mathbf{R}^{2}$ vérifient :

$$
\gamma_{1}(t)=(t, 0), \quad \gamma_{2}(t)=(0, t) .
$$

Si $\Gamma$ est un lacet sur une surface $M$, on notera $[\Gamma] \in \mathrm{H}_{1}(\mathbf{M}, \mathbf{Z})$ la classe d'homologie de $\Gamma$. On obtient une forme alternée $\wedge$ sur $\mathrm{H}_{1}(\mathrm{M}, \mathbf{R})$ à partir de l'égalité $[\Gamma] \wedge\left[\Gamma^{\prime}\right]=\Gamma \wedge \Gamma^{\prime}$, définie pour des lacets $\Gamma$ et $\Gamma^{\prime}$.

On appellera droite (resp. demi-droite négative, demi-droite positive, segment, cercle) d'une surface $\mathrm{M}$ tout plongement topologique propre de $\mathbf{R}$ (resp. ] - $\infty, 0$ ], $\left[0,+\infty\left[,[0,1], \mathbf{T}^{1}\right)\right.$ ou plus précisément toute classe d'équivalence d'un tel plongement par reparamétrage préservant l'orientation. Un tel objet est alors déterminé par son image et une orientation. On utilisera là encore souvent le mot droite, demi-droite, segment ou cercle, pour désigner l'image du plongement.

Grâce au théorème de Schoenflies, si $\Gamma$ est une droite du plan $\mathbf{R}^{2}$, on peut construire un homéomorphisme $h$ de $\mathbf{R}^{2}$ préservant l'orientation tel que $h \circ \Gamma(t)=$ $(0, t)$, pour tout $t \in \mathbf{R}$. Le demi-plan fermé $h^{-1}([0,+\infty[\times \mathbf{R})$ est alors indépendant de $h$, on dit que c'est le demi-plan à droite de $\Gamma$ et on le note $\mathrm{D}(\Gamma)$. De même, on note $\left.\left.\mathrm{G}(\Gamma)=h^{-1}(]-\infty, 0\right] \times \mathbf{R}\right)$ le demi-plan fermé à gauche de $\Gamma$. Remarquons que $\mathrm{D}(\Gamma) \backslash \Gamma$ et $\mathrm{G}(\Gamma) \backslash \Gamma$ sont les composantes connexes de $\mathbf{R}^{2} \backslash \Gamma$. De même, on peut définir pour tout cercle $\Gamma$, les ensembles $\mathrm{D}(\Gamma)$ et $\mathrm{G}(\Gamma)$, l'un est un disque fermé et l'autre un anneau non borné.

On dira que deux droites $\Gamma$ et $\Gamma^{\prime}$ du plan n'ont pas d'intersection transverse si $\Gamma \subset \mathrm{D}\left(\Gamma^{\prime}\right)$ ou $\Gamma \subset \mathrm{G}\left(\Gamma^{\prime}\right)$. Il est facile de voir que cette relation est symétrique. 
Plus précisément, $\Gamma$ et $\Gamma^{\prime}$ n'ont pas d'intersection transverse si et seulement si l'une des quatre propriétés suivantes est vérifiée :

- $\mathrm{D}(\Gamma) \subset \mathrm{D}\left(\Gamma^{\prime}\right)$ (et alors $\left.\mathrm{G}\left(\Gamma^{\prime}\right) \subset \mathrm{G}(\Gamma)\right)$;

- $\mathrm{D}\left(\Gamma^{\prime}\right) \subset \mathrm{D}(\Gamma)$ (et alors $\left.\mathrm{G}(\Gamma) \subset \mathrm{G}\left(\Gamma^{\prime}\right)\right)$;

- $\mathrm{D}(\Gamma) \cup \mathrm{D}\left(\Gamma^{\prime}\right)=\mathbf{R}^{2}$ (et alors $\left.\mathrm{G}(\Gamma) \cap \mathrm{G}\left(\Gamma^{\prime}\right) \subset \Gamma \cap \Gamma^{\prime}\right)$;

- $\mathrm{G}(\Gamma) \cup \mathrm{G}\left(\Gamma^{\prime}\right)=\mathbf{R}^{2}$ (et alors $\left.\mathrm{D}(\Gamma) \cap \mathrm{D}\left(\Gamma^{\prime}\right) \subset \Gamma \cap \Gamma^{\prime}\right)$.

Les feuilletages que nous rencontrerons par la suite seront toujours des feuilletages topologiques orientés. Là encore, nous oublierons souvent de le préciser. On peut définir naturellement l'ensemble $\alpha$-limite $\alpha(\phi) \subset \mathrm{M}$ et l'ensemble $\omega$-limite $\omega(\phi) \subset \mathrm{M}$ d'une feuille $\phi$ d'un feuilletage orienté $\mathscr{F}$ défini sur une partie ouverte U d'une surface M. On dira qu'une feuille $\phi$ joint $z_{0} \in \mathrm{M}$ à $z_{1} \in \mathrm{M}$, ou encore est une connexion entre $z_{0}$ et $z_{1}$, si $\alpha(\phi)=z_{0}$ et $\omega(\phi)=z_{1}$. Un cycle est une famille finie $\left(\phi_{i}\right)_{i \in \mathbf{Z} / n \mathbf{Z}}$ de connexions telle que $\omega\left(\phi_{i}\right)=\alpha\left(\phi_{i+1}\right)$ pour tout $i \in \mathbf{Z} / n \mathbf{Z}$.

On dira par définition qu'un arc $\gamma: \mathrm{I} \rightarrow \mathbf{R}$ est positivement transverse à un feuilletage $\mathscr{F}$ défini sur une surface $\mathrm{M}$ si, pour tout $t_{0} \in \mathrm{I}$, il existe un voisinage $\mathrm{U}$ de $\gamma\left(t_{0}\right)$ dans $\mathbf{M}$ et un homéomorphisme préservant l'orientation $h$ entre $\mathrm{U}$ et un ouvert $\mathrm{V}$ de $\mathbf{R}^{2}$ qui envoie le feuilletage $\mathscr{F}$ sur le feuilletage vertical, orienté suivant les $y$ croissants, et tel que l'application $t \mapsto p_{1}(h(\gamma(t)))$ soit strictement croissante au voisinage de $t_{0}$, où $p_{1}$ désigne la première projection.

\section{Introduction}

Le théorème de translation de Brouwer ([Br]) énonce que si $f$ est un homéomorphisme de $\mathbf{R}^{2}$ qui préserve l'orientation et qui n'a pas de point fixe, alors par tout point du plan passe une droite $\Gamma$ telle que

$$
f(\Gamma) \subset \mathrm{D}(\Gamma) \backslash \Gamma, \quad f^{-1}(\Gamma) \subset \mathrm{G}(\Gamma) \backslash \Gamma .
$$

Un tel homéomorphisme est appelé un homéomorphisme de Brouwer et $\Gamma$ une droite de Brouwer. Remarquons que $f(\mathrm{D}(\Gamma)) \subset \mathrm{D}(\Gamma)$ et que l'ensemble

$$
\bigcup_{n \geq 0} f^{-n}(\mathrm{D}(\Gamma)) \backslash \bigcap_{n \geq 0} f^{n}(\mathrm{D}(\Gamma))=\bigsqcup_{k \in \mathbf{Z}} f^{k}(\mathrm{D}(\Gamma) \backslash f(\mathrm{D}(\Gamma)))
$$

est un disque ouvert invariant sur lequel l'application $f$ est conjuguée à la translation du plan $\mathrm{T}:(x, y) \mapsto(x+1, y)$. Le fait que l'on puisse recouvrir le plan par des disques invariants où $f$ est conjugué à une translation implique que $f$ n'a pas de point périodique et plus généralement que tout point est errant. Les démonstrations du théorème de translation de Brouwer (voir Guillou [Gu] pour une preuve actualisée de ce théorème, ou encore Franks [Fr2] ou [LS]) utilisent 
indirectement le lemme de translation de Brouwer : un homéomorphisme de Brouwer n'a pas de point périodique (voir Brown [Bn] ou Fathi [Fa]). On peut, en modifiant pertinemment $f$ sans rajouter de point fixe et en appliquant alors ce lemme, montrer qu'un homéomorphisme de Brouwer $f$ n'a pas de point errant. On peut même démontrer la propriété de non récurrence plus forte suivante : on ne peut pas trouver de suite $\left(\mathrm{U}_{i}\right)_{i \in \mathbf{Z} / n \mathbf{Z}}$ de disques ouverts disjoints deux-à-deux et disjoints de leur image par $f$ et de suite d'entiers $\left(p_{i}\right)_{i \in \mathbf{Z} / n \mathbf{Z}}$ strictement positifs, tels que $f^{p_{i}}\left(\mathrm{U}_{i}\right) \cap \mathrm{U}_{i+1} \neq \emptyset$, pour tout $i \in \mathbf{Z} / n \mathbf{Z}$. Ce résultat, appelé classiquement le lemme de Franks (voir [Frl]), sera à la base de cet article.

Dans certains cas, la démonstration du théorème de translation de Brouwer est facile et la droite de Brouwer passant par un point $z$ peut être décrite explicitement. Ainsi, si $f$ est le temps 1 d'un flot défini par un champ de vecteurs $\xi$ de classe $\mathrm{C}^{1}$ sans singularité, il suffit de prendre l'orbite de $z$ pour le champ $\zeta$, image de $\xi$ par une rotation d'angle $\pi / 2$. Dans ce cas, on peut feuilleter le plan par des droites de Brouwer (on peut d'ailleurs, par un argument d'approximation choisir le feuilletage de classe $\mathrm{C}^{\infty}$ ). Un autre cas où l'existence d'un feuilletage en droites de Brouwer se démontre facilement est le cas où $f$ est un difféomorphisme $\mathrm{C}^{1}$-proche de l'identité, plus précisément le cas où la différentielle $\mathrm{D} f(z)$ en chaque point n'a pas de valeur propre réelle négative (voir [LeC5]). On peut naturellement se demander si le plan peut être feuilleté par des droites de Brouwer, pour un homéomorphisme de Brouwer quelquonque. La réponse est oui (voir [LeC5]) :

Théorème 1.1. - Soit $f$ un homéomorphisme de Brouwer. Il existe un feuilletage topologique orienté $\mathscr{F}$ du plan dont toute feuille est une droite de Brouwer de $f$.

Remarquons que pour tout point $z_{0} \in \mathbf{R}^{2}$, il existe un arc positivement transverse à $\mathscr{F}$ qui joint $z_{0}$ à $f\left(z_{0}\right)$. En effet, si on note $\mathrm{W}$ l'ensemble des points $z \in \mathbf{R}^{2}$ qui peuvent être joints par un arc issu de $z_{0}$, positivement transverse à $\mathscr{F}$, on remarque que W est une partie ouverte, dont la frontière est formée de la feuille $\phi_{0}$ contenant $z_{0}$ et éventuellement de feuilles $\phi$ telles que $\overline{\mathrm{W}} \subset \mathrm{D}(\phi)$. Si le point $f\left(z_{0}\right)$ n'appartient pas à W, il appartient à l'un des ensembles $\mathrm{G}(\phi)$. On en déduit que $z_{0}$ appartient à l'intérieur de $\mathrm{G}(\phi)$, puisque $\phi$ est une droite de Brouwer, ce qui est impossible. Réciproquement, un feuilletage qui vérifie la propriété précédente est clairement un feuilletage en droites de Brouwer.

Il est à noter que le feuilletage $\mathscr{F}$ n'a aucune raison d'être invariant par $f$. Il n'est pas difficile en effet de démontrer, dans le cas où $\mathscr{F}$ est invariant, que par tout point $z$ passe une droite invariante, appelée classiquement une trajectoire propre. Or, on connait des exemples d'homéomorphismes de Brouwer qui n'admettent aucune trajectoire propre (voir Brown, Slaminka, Transue [BST]). 
Le but de la première partie de cet article est de donner une version équivariante du théorème précédent :

Théorème 1.2. - Soit G un groupe discret d'homéomorphismes du plan préservant l'orientation, agissant librement et proprement. Soit $f$ un homéomorphisme de Brouwer commutant avec les éléments de G. Il existe alors un feuilletage topologique orienté du plan, invariant sous l'action de $\mathrm{G}$, dont toute feuille est une droite de Brouwer de $f$.

Si on veut appliquer le théorème au groupe des itérés de $f$, c'est-à-dire si on cherche un feuilletage en droites de Brouwer invariant par $f$, il faut que l'action des itérés soit propre, c'est-à-dire que $f$ soit conjugué à une translation, et le résultat est alors trivial. L'idée de la démonstration du théorème 1.2 s'inspire de celle du théorème 1.1 donnée dans [LeC5] et utilise, là encore, la notion de décomposition libre sur une surface. Cette notion a été introduite par Flucher [Flu] pour montrer l'existence d'un deuxième point fixe dans la version topologique du théorème de Conley-Zehnder en dimension 2, puis dans [LeC2] pour obtenir un troisième point fixe. Elle a été utilisée ensuite dans [LS] pour établir une preuve du théorème de translation de Brouwer, développée par Sauzet [Sa] dans sa thèse et appliquée il y a peu par Le Roux [LeR] dans son étude de la dynamique d'un homéomorphisme du plan au voisinage d'un point fixe d'indice de Lefschetz $\neq 1$. L'idée de la démonstration du théorème 1.1 est de partir d'un pavage $\mathscr{D}$ $\mathrm{du}$ plan en briques tel que toute brique est disjointe de son image par $f$ et qui vérifie la propriété de maximalité suivante : un pavage plus grossier (i.e. dont les briques sont réunions de briques de $\mathscr{D}$ ) ne vérifie plus cette propriété. Il existe alors une orientation naturelle du squelette de cette décomposition, c'est-à-dire de la réunion des bords de briques, uniquement définie par la propriété suivante : l'image par $f$ de la brique à gauche d'une arête rencontre la brique à droite de cette arête. On peut alors recouvrir le squelette de cette décomposition par des droites de Brouwer dont l'orientation est compatible avec celle du squelette et qui n'ont pas d'intersection transverse deux à deux. On peut alors munir cet ensemble de droites $\mathscr{B}^{*}$ d'une topologie naturelle, généralement non séparée, mais localement compacte et d'un ordre naturel. En feuilletant convenablement chacune des briques, on peut étendre notre famille $\mathscr{B}^{*}$ en une famille de droites de Brouwer recouvrant le plan tout entier et définissant un quasi-feuilletage. Soyons plus précis. Un point à l'intérieur d'une brique appartient à une unique droite et la famille étendue définit un feuilletage au voisinage du point ; un point sur une arête qui n'est pas un sommet appartient à plusieurs droites mais celles-ci coïncident avec l'arête au voisinage du point, la famille étendue définit encore un feuilletage local ; tout sommet est singulier. Un argument de désingularisation permet alors de séparer les droites ayant une arête commune et d'obtenir un feuilletage du plan en droites de Brouwer dont une partie fermée est isomorphe à $\mathscr{B}^{*}$. Ici, il faudra bien sûr rendre 
équivariant cette méthode. Nous partirons d'un pavage $\mathscr{D} \mathrm{du}$ plan en briques qui est G-équivariant, tel que toute brique est disjointe de son image par $f$, et qui est maximal pour cette propriété parmi les pavages équivariants. En reprenant et complètant des arguments utilisés par Sauzet dans sa thèse, nous verrons qu'il est possible d'orienter naturellement le squelette de la décomposition. Le problème principal est alors de recouvrir ce squelette par une famille équivariante de droites de Brouwer qui n'ont pas d'intersection transverse. Ceci est facile à faire dans le cas où le groupe $\mathrm{G}$ est abélien, les arguments sont ensemblistes et utilisent plus ou moins le fait que tout groupe abélien sans torsion est ordonnable. C'est beaucoup plus difficile dans le cas où $G$ n'est pas abélien et nous aurons besoin d'arguments topologiques plus fins. Remarquons que le cas où $G$ est abélien correspond au cas où $G$ est isomorphe à $\mathbf{Z}$ ou à $\mathbf{Z}^{2}$ et que la surface $M$ est alors homéomorphe à l'anneau $\mathbf{T}^{1} \times \mathbf{R}$ ou au tore $\mathbf{T}^{2}$.

Grâce à la remarque qui suit le théorème 1.1, on peut, en passant au revêtement universel, énoncer le théorème 1.2 sous la forme équivalente suivante :

Théorème 1.3. - Soit $\mathrm{M}$ une surface et $\left(\mathrm{F}_{t}\right)_{t \in[0,1]}$ une isotopie dans $\mathrm{M}$ joignant l'identité à un homéomorphisme $\mathrm{F}$. Pour tout $z \in \mathrm{M}$, on défnit l'arc $\gamma_{z}: t \mapsto \mathrm{F}_{t}(z)$. On suppose que F n'a pas de point fixe z contractile, c'est-à-dire de point fixe z tel que $\gamma_{z}$ soit un lacet homotope à 0 . Il existe alors un feuilletage topologique orienté $\mathscr{F}$ sur $\mathrm{M}$ et pour tout $z \in \mathrm{M}$, un arc positivement transverse à $\mathscr{F}$ joignant $z$ à $\mathrm{F}(z)$ qui est homotope, à extrémités fixées, à l'arc $\gamma_{z}$.

Nous dirons, par abus de langage, que le feuilletage $\mathscr{F}$ et l'isotopie $\left(\mathrm{F}_{t}\right)_{t \in[0,1]}$ sont transverses. Chaque feuille est en quelque sorte "poussée vers la droite" au temps un de l'isotopie. Remarquons que si $\mathrm{M}$ est de type infini, ou alors si $\mathrm{M}$ est de type fini et si sa caractéristique d'Euler est négative, la classe d'homotopie de l'arc $\gamma_{z}$ est indépendante de l'isotopie, elle est bien déterminée par F (voir [Haml] et [Ham2] par exemple). Si M est un anneau ou un tore, la classe de $\gamma_{z}$ est déterminée par le choix d'un relèvement $f$ de $\mathrm{F}$ au revêtement universel. Remarquons également qu'on ne suppose aucune hypothèse de finitude sur le type ni même sur le genre de $\mathrm{M}$.

Dans la seconde partie de cet article, nous verrons des applications du théorème 1.3 à l'étude des homéomorphismes de surfaces. En particulier, nous verrons comment la construction d'un feuilletage transverse nous permet de retrouver rapidement certains résultats connus sur les homéomorphismes conservatifs de surfaces. Ainsi nous donnerons une nouvelle preuve du résultat suivant de Franks [Fr5] :

Théorème 1.4. - Un homéomorphisme $\mathrm{F}$ de la sphère $\mathrm{S}^{2}$ qui préserve une mesure de probabilité dont le support est $\mathrm{S}^{2}$ et qui a au moins trois points fixes a une infinité d'orbites périodiques. 
Nous démontrerons également (la notion de vecteur de rotation est rappelée au début de la section 8) :

Théorème 1.5. - Soit $\mathrm{M}$ une surface compacte de genre $g \geq 1$ et $\left(\mathrm{F}_{t}\right)_{t \in[0,1]}$ une isotopie dans $\mathrm{M}$ joignant l'identité à un homéomorphisme $\mathrm{F}$. On suppose que $\mathrm{F}$ préserve une mesure borélienne de probabilité dont le support est $\mathrm{M}$ et que le vecteur de rotation de cette mesure est nul.

i) Il existe alors au moins trois points fixes contractiles.

ii) Si l'ensemble des points fixes contractiles est fini, il existe des points périodiques contractiles de périodes arbitrairement grandes.

Un cas particulier d'application du théorème est le cas où $\mathrm{F}$ est le temps 1 d'une isotopie hamiltonienne. Soit $(\mathrm{M}, \omega)$ une variété symplectique compacte et $\mathrm{H}: \mathbf{R} \times \mathrm{M} \rightarrow \mathbf{R}$ une fonction de classe $\mathrm{C}^{2}$, vérifiant

$$
\mathrm{H}(t+1, z)=\mathrm{H}(t, z) .
$$

Pour tout $t \in \mathbf{R}$, notons $\mathrm{H}_{t}: \mathrm{M} \rightarrow \mathbf{R}$ la fonction partielle en $z$ et $\mathrm{X}_{t}$ le champ de vecteurs hamiltonien associé à $\mathrm{H}_{t}$, c'est-à-dire tel que pour tout champ de vecteurs Y sur M, on ait :

$$
\omega\left(\mathrm{X}_{t}, \mathrm{Y}\right)=d \mathrm{H}_{t}(\mathrm{Y})
$$

Notons alors $\left(\mathrm{F}_{t}\right)_{t \in \mathbf{R}}$ l'isotopie définie par :

$$
\mathrm{F}_{0}=\operatorname{Id}_{\mathrm{M}}, \frac{d}{d t} \mathrm{~F}_{t}(z)=\mathrm{X}_{t}\left(\mathrm{~F}_{t}(z)\right)
$$

Nous obtenons une isotopie hamiltonienne vérifiant :

$$
\mathrm{F}_{t}=\mathrm{F}_{t-1} \circ \mathrm{F}_{1}=\mathrm{F}_{1} \circ \mathrm{F}_{t-1} \text {. }
$$

Les points fixes contractiles de $\mathrm{F}_{1}$ correspondent à des orbites périodiques de période 1 de notre équation différentielle telles que le lacet $t \mapsto \mathrm{F}_{t}(z)$ soit homotope à zéro. La conjecture d'Arnold prédit que le nombre de points fixes contractiles est toujours minoré par le nombre minimum de points critiques que doit possèder toute fonction définie sur $\mathrm{M}$ (voir [HZ] pour un historique du problème). Le cas des surfaces de genre $\geq 1$ (où le nombre minimum est trois) avait été résolu par Floer [Flo] et Sikorav [Sik]. On dira qu'un homéomorphisme vérifiant les hypothèses du théorème 1.5 est un homéomorphisme hamiltonien. L'assertion i) est une version topologique de ce qui précède. Une démonstration en avait été donnée dans le cas du tore [LeC2], elle est due dans le cas général à Matsumoto [M]. 
Le problème de l'existence d'une infinité d'orbites périodiques contractiles, à savoir l'assertion ii), restait ouvert, même pour les difféomorphismes. La recherche d'une infinité de points périodiques contractiles en dimension supérieure est un problème difficile et les résultats connus dans cette direction (Salamon-Zehnder [SZ], Schwarz $[\mathrm{Scz}]$, ...) supposent tous une propriété plus ou moins forte de généricité. Un résultat tout récent de Franks et Handel $[\mathrm{FH}]$ affirme qu'un difféomorphisme hamiltonien différent de l'identité a des orbites périodiques de périodes arbitrairement grandes (mais non nécessairement contractiles). Ici on ne suppose pas de condition de différentiabilité. Dans le cas particulier d'un difféomorphisme, l'assertion ii) du théorème 1.5 énonce un résultat plus fort sous une hypothèse plus forte.

Expliquons dans les grandes lignes pour quelles raisons le théorème 1.3 nous donne l'assertion i) du théorème 1.5. Le théorème 1.3 nous dit que tout homéomorphisme $\mathrm{F}$, temps un d'une isotopie, admet un feuilletage transverse $\mathscr{F}$, dès qu'une obstruction évidente (présence d'une trajectoire fermée contractile) est levée. On peut s'attendre à ce que la dynamique du feuilletage soit "transverse" à celle de l'homéomorphisme et étudier ce feuilletage à partir des informations connues sur F. Par exemple, donnons-nous un homéomorphisme F d'une surface $\mathrm{M}$, isotope à l'identité, ayant un nombre fini de points fixes et considérons un ensemble $\mathrm{X} \subset \operatorname{Fix}(\mathrm{F})$ qui est non enlacé, c'est-à-dire tel que $\mathrm{F}_{\mid \mathrm{M} \backslash \mathrm{X}}$ soit isotope à l'identité, et maximal (au sens de l'inclusion) pour cette propriété de non enlacement. Le théorème 1.3 s'applique alors à $\mathrm{F}_{\mid \mathrm{M} \backslash \mathrm{X}}$ et on obtient un feuilletage $\mathscr{F}$ sur $\mathrm{M}$ dont $\mathrm{X}$ est l'ensemble des singularités. Si F est un homéomorphisme hamiltonien d'une surface compacte, nous verrons que le feuilletage $\mathscr{F}$ est de type gradient et que X possède donc au moins trois éléments. Soyons plus précis. Habituellement, la recherche des points fixes contractiles d'un difféomorphisme hamiltonien se ramène à celle des points critiques d'une fonction L définie sur un espace de dimension finie ou infinie, ces points critiques s'obtenant par l'étude dynamique du gradient de la fonction. La valeur de $\mathrm{L}$ aux points critiques a un sens intrinsèque (à une constante près), c'est l'action L définie par l'isotopie aux points fixes correspondants. La quantité $\mathrm{L}\left(z_{1}\right)-\mathrm{L}\left(z_{0}\right)$ mesure l'aire balayée le long de l'isotopie. Dans le cas d'un difféomorphisme hamiltonien d'une surface dont l'ensemble des points fixes contractiles est fini, la fonction $\mathrm{L}$ prend au moins trois valeurs sur cet ensemble. Ici le feuilletage est défini directement sur la surface et donné a priori par le théorème 1.3. Nous verrons comment construire naturellement à partir d'une famille finie d'orbites de $\mathrm{F}$, un lacet $\Gamma \subset \mathrm{M} \backslash \Gamma$ positivement transverse au feuilletage, homologue à zéro et séparant la variété en composantes connexes topologiquement triviales (dont l'adhérence est un disque fermé) contenant au maximum un point de $\mathrm{X}$. Ce lacet étant homologue à zéro définit naturellement une fonction à valeurs entières $\Lambda$ sur $M \backslash \Gamma$, à une constante près. La quantité $\Lambda\left(z_{1}\right)-\Lambda\left(z_{0}\right)$ représente l'intersection algébrique $\Gamma^{\prime} \wedge \Gamma$, où $\Gamma^{\prime}$ est un arc joignant $z_{0}$ à $z_{1}$. On 
en déduit que $\Lambda$ décroit le long des feuilles et n'est constante sur aucune feuille. Chacune des feuilles est alors issue d'un point $z_{0} \in \mathrm{X}$ et aboutit à un autre point de $z_{1} \in \mathrm{X}$, où $\Lambda\left(z_{1}\right)<\Lambda\left(z_{0}\right)$. On montre alors que $\Lambda$ prend au moins trois valeurs sur $\mathrm{X}$. La fonction $\Lambda$ est donc une version discrète de la fonction $\mathrm{L}$. Il est d'ailleurs possible de construire le lacet $\Gamma$ de telle façon que $\Lambda$ soit, à un facteur multiplicatif près, une approximation aussi fine que l'on veut de $\mathrm{L}$ sur $\mathrm{X}$.

L'obtention d'infinité d'orbites périodiques contractiles est plus difficile, l'idée (qu'on retrouve également dans la preuve du théorème 1.4) est de construire à partir d'un feuilletage transverse, un homéomorphisme d'un anneau ouvert ayant deux points de vecteurs de rotation distincts et vérifiant la propriété d'intersection suivante : tout lacet simple non homotope à zéro rencontre son image. Un tel homéomorphisme possède d'après Franks [Frl] une infinité d'orbites périodiques. La preuve de ce qui apparait comme une généralisation du théorème géométrique de Poincaré-Birkhoff (au moins pour l'existence d'un point fixe) se démontre en utilisant l'absence de châne fermée de disques libres pour un homéomorphisme de Brouwer. Nous en donnerons d'ailleurs une démonstration directe à partir du théorème 1.3 .

L'existence d'un lien entre le théorème de Poincaré-Birkhoff (qui peut être vu comme un problème de dynamique hamiltonienne) et le théorème de translation de Brouwer est ancien et se retrouve déjà chez Kerékjártó $[K]$, qui s'était demandé si le premier théorème ne se déduisait pas d'une forme équivariante du second. La démonstration donnée par Guillou [Gu] du théorème de translation de Brouwer contient une version équivariante qui implique le théorème de PoincaréBirkhoff. Rappelons, à l'inverse, qu'une preuve du théorème 1.3 était connue dans le cas d'un difféomorphisme $\mathrm{F}$ de $\mathbf{T}^{2}$, par des méthodes issues de la géométrie symplectique, à savoir en décomposant $\mathrm{F}$ en difféomorphismes déviant la verticale [LeC1]. Des applications de ce résultat à l'étude des ensembles de rotation des difféomorphismes du tore isotopes à l'identité ont été donnés dans [LeC1], qui n'utilisent plus la différentiabilité, une fois le feuilletage obtenu (Théorèmes 5.1 et 5.2 , p. 106). La preuve de ces résultats restent donc valables pour les homéomorphismes. Remarquons également que les cas cités au début de la section où le théorème de translation de Brouwer (temps un d'un champ de vecteurs indépendant du temps, cas proche de l'identité) sont les cas dans le cadre hamiltonien où la conjecture d'Arnold se démontre facilement et s'obtient directement sur la variété.

Expliquons le plan de l'article. La première partie commence à la section 2 et se termine à la section 7. En section 2, nous introduirons les décompositions libres des surfaces et rappelerons les travaux de Sauzet sur le sujet. En section 3, nous énoncerons la propriété fondamentale de recouvrement du squelette de la décomposition par une famille équivariante de droites de Bouwer sans intersections transverses et nous en donnerons une démonstration dans le cas où le groupe est 
abélien. Nous expliquerons, dans la section 4, comment cette propriété nous donne le théorème 1.2. Nous montrerons le résultat fondamental dans le cas où $G$ n'est pas abélien en section 7 . Nous aurons besoin de résultats préliminaires : un résultat de prolongement qui sera montré en section 5, une propriété topologique d'indice qui sera expliquée en section 6. La seconde partie commence à la section 8 et se termine à la section 11. Dans la section 8, nous rappelons la définition du vecteur de rotation d'un point et d'une mesure invariante, pour un homéomorphisme donné comme temps 1 d'une isotopie. Nous verrons comment transcrire l'existence d'un vecteur de rotation sur un feuilletage transverse. En section 9, nous donnerons une preuve du théorème 1.4 et de résultats autour de celui-ci. En section 10 nous montrerons l'assertion i) du théorème 1.5, nous détaillerons les arguments cités plus haut dans cette introduction et décrirons la dynamique $\mathrm{du}$ feuilletage transverse $\mathscr{F}$. L'assertion ii) du théorème 1.5 sera prouvée dans la section 11 .

Je tiens à remercier fortement le referee pour sa lecture attentive de l'article. Ses très nombreux conseils ont permi de beaucoup améliorer la rédaction du texte original.

\section{Démonstration de l'existence d'un feuilletage transverse}

\section{Décompositions en briques}

On va rappeler puis complèter dans la section 2 les travaux de Sauzet [Sa].

\section{Définition des décompositions}

On appellera décomposition en briques d'une surface $\mathrm{M}$ la donnée d'un ensemble stratifié $\Sigma(\mathscr{D})$ de dimension un, appelé squelette de la décomposition $\mathscr{D}$, avec une sous-variété de dimension zéro $\mathrm{S}$ tel que de tout sommet $\sigma \in \mathrm{S}$ soient issues (localement) exactement trois arêtes. On distinguera les arêtes ouvertes, qui sont les composantes connexes de $\Sigma(\mathscr{D}) \backslash \mathrm{S}$, des arêtes fermées, qui sont leurs adhérences. De même, on distinguera les briques ouvertes qui sont les composantes connexes de $\mathrm{M} \backslash \Sigma(\mathscr{D})$ des briques fermées qui sont leurs adhérences. L'absence de qualificatif aux mots arête et brique signifiera par la suite que l'on considère les objets fermés. On notera A l'ensemble des arêtes et $\mathrm{B}$ l'ensemble des briques et on écrira $\mathscr{D}=(\mathrm{S}, \mathrm{A}, \mathrm{B})$ pour désigner une décomposition en briques. Les extrémités d'une arête $\alpha$ ouverte ou fermée sont les sommets qui appartiennent à $\bar{\alpha}$. Remarquons qu'une arête $\alpha$ est un segment si elle a deux extrémités ; une demi-droite ou un cercle si elle en a une ; une droite ou un cercle si elle n'en a pas. L'intérieur int $(\alpha)$ de $\alpha$ est par définition l'arête ouverte associée à $\alpha$, c'est-à-dire l'arête $\alpha$ privée de 
ses extrémités. Les briques adjacentes à $\alpha \in \mathrm{A}$ (resp. $\sigma \in \mathrm{S}$ ) sont les briques qui contiennent $\alpha$ (resp. $\sigma$ ), il y en a au plus deux (resp. trois).

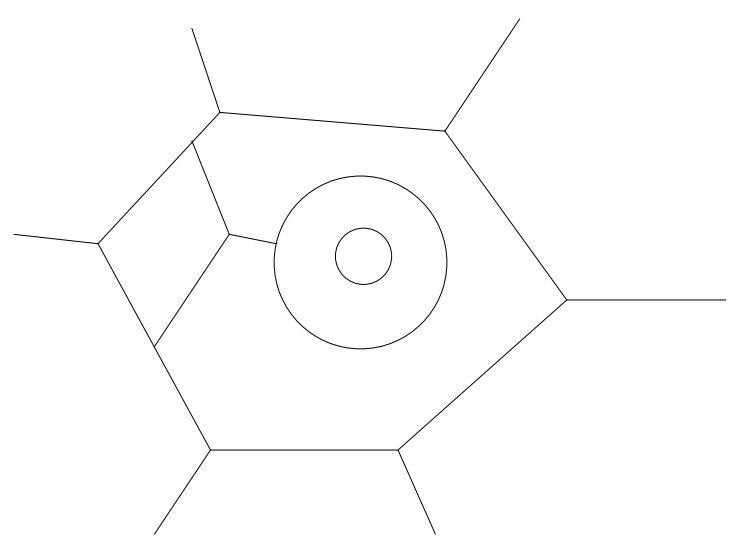

Soient $\mathscr{D}=(\mathrm{S}, \mathrm{A}, \mathrm{B})$ et $\mathscr{D}^{\prime}=\left(\mathrm{S}^{\prime}, \mathrm{A}^{\prime}, \mathrm{B}^{\prime}\right)$ deux décompositions en briques d'une surface $\mathrm{M}$. On dira que $\mathscr{D}^{\prime}$ est une sous-décomposition de $\mathscr{D}$ si $\Sigma\left(\mathscr{D}^{\prime}\right)$ est inclus dans $\Sigma(\mathscr{D})$. Tout sommet de $\mathscr{D}^{\prime}$ est un sommet de $\mathscr{D}$, toute arête de $\mathscr{D}^{\prime}$ est réunion d'arêtes de $\mathscr{D}^{\prime}$ et toute brique de $\mathscr{D}^{\prime}$ réunion de briques de $\mathscr{D}$. Cependant, les ensembles B et $\mathrm{B}^{\prime}$ peuvent être égaux sans que $\mathscr{D}$ et $\mathscr{D}^{\prime}$ le soient, il suffit par exemple de rajouter à $\Sigma\left(\mathscr{D}^{\prime}\right)$ un cercle contenu dans une brique ouverte $\beta$ de $\mathscr{D}^{\prime}$ et qui ne sépare pas $\beta$.

Soit $\pi: M \rightarrow \widetilde{M}$ un revêtement galoisien sur $\widetilde{M}$. Si $\widetilde{\mathscr{D}}=(\widetilde{\mathrm{S}}, \widetilde{\mathrm{A}}, \widetilde{\mathrm{B}})$ est le squelette d'une décomposition en briques de $\tilde{\mathrm{M}}$, l'ensemble $\pi^{-1}(\Sigma(\widetilde{\mathscr{D}}))$ est le squelette d'une décomposition en briques $\mathscr{D}=(\mathrm{S}, \mathrm{A}, \mathrm{B})$ de $\mathrm{M}$, la décomposition relevée. On écrira $\mathscr{D}=\pi^{-1}(\widetilde{D})$. Si $\mathrm{G}$ est le groupe des automorphismes du revêtement, alors les ensembles $\mathrm{S}$, A et B sont invariants sous l'action de $\mathrm{G}$, la décomposition est G-équivariante (on dira plus simplement équivariante s’il n'y a pas d'ambiguité).

Soit $\mathscr{D}=(\mathrm{S}, \mathrm{A}, \mathrm{B})$ une décomposition en briques de $\mathrm{M}$. On dira qu'une partie $\mathrm{X}$ de $\mathrm{B}$ est connexe si, pour toutes briques $\beta, \beta^{\prime} \in \mathrm{X}$, il existe une suite $\left(\beta_{i}\right)_{0 \leq i \leq n}$ d'éléments de $\mathrm{X}$, où $\beta_{0}=\beta$ et $\beta_{n}=\beta^{\prime}$, telle que $\beta_{i}$ et $\beta_{i+1}$ sont adjacentes si $i \in\{0, \ldots, n-1\}$, c'est-à-dire contiennent une arête commune. On identifiera par la suite une partie $\mathrm{X}$ de $\mathrm{B}$ et la partie fermée de $\mathrm{M}$, réunion des briques de $\mathrm{X}$. Il n’y aura généralement pas d'ambiguité : si on parle de l'intérieur Int(X) de X, de sa frontière $\partial \mathrm{X}$, alors $\mathrm{X}$ est une partie de $\mathrm{M}$. On lèvera l'ambiguité, si nécessaire, en précisant $\mathrm{X} \subset \mathrm{B}$ ou $\mathrm{X} \subset \mathrm{M}$. Remarquons que la connexité de $\mathrm{X} \subset \mathrm{B}$ équivaut à celle de $\mathrm{X} \subset \mathrm{M}$ et qu'elle équivaut également à celle de $\operatorname{Int}(\mathrm{X})$. Remarquons que la famille des composantes connexes de $\mathrm{X} \subset \mathrm{M}$ définit une partition de $\mathrm{X} \subset \mathrm{B}$ en parties connexes de B. Remarquons enfin (c'est une propriété importante des 
décompositions en briques) que $\partial \mathrm{X}$ est une variété topologique de dimension 1 pour tout $\mathrm{X} \subset \mathrm{B}$.

Si $\mathscr{D}=(\mathrm{S}, \mathrm{A}, \mathrm{B})$ est une décomposition en briques de $\mathrm{M}$ et $\left(\mathrm{X}_{i}\right)_{i \in \mathrm{I}}$ une partition de B en parties connexes, alors $\bigcup_{i \in \mathrm{I}} \partial \mathrm{X}_{i}$ est le squelette d'une sous-décomposition $\mathscr{D}^{\prime}=\left(\mathrm{S}^{\prime}, \mathrm{A}^{\prime}, \mathrm{B}^{\prime}\right)$ de $\mathscr{D}$ dont les briques sont les $\mathrm{X}_{i}$. Remarquons que tout arête $\alpha \in \mathrm{A}^{\prime}$ est adjacente à exactement deux briques et que tout sommet $\sigma \in \mathrm{S}^{\prime}$ est adjacent à exactement trois briques. Remarquons également que toute arête qui est un cercle n'a pas d'extrémité. Un cas particulier de sous-décomposition de $\mathscr{D}$ est la décomposition obtenue à partir de la partition triviale de $\mathrm{B}$, c'est-à-dire de la partition en points $(\{\beta\})_{\beta \in \mathrm{B}}$. Si cette décomposition, qui a mêmes briques que $\mathscr{D}$, coïncide avec $\mathscr{D}$, on dira que $\mathscr{D}$ est une décomposition remplie.

On appellera $\mathscr{D}$-cercle (resp. $\mathscr{D}$-droite) tout cercle (resp. droite) contenu(e) dans $\Sigma(\mathscr{D})$; on appellera $\mathscr{D}$-demi-droite toute demi-droite contenue dans $\Sigma(\mathscr{D})$ dont l'extrémité est dans $\mathrm{S}$; on appellera $\mathscr{D}$-segment tout segment contenu dans $\Sigma(\mathscr{D})$ dont les extrémités sont dans $\mathrm{S}$.

Action d'un homéomorphisme sur une décomposition

Soit $f$ un homéomorphisme de M. On définit naturellement une application

$$
\varphi: \mathscr{P}(\mathrm{B}) \rightarrow \mathscr{P}(\mathrm{B})
$$

en posant :

$$
\begin{aligned}
\varphi(\mathrm{X}) & =\left\{\beta \in \mathrm{B} \mid \text { il existe } \beta^{\prime} \in \mathrm{X} \text { tel que } \beta \cap f\left(\beta^{\prime}\right) \neq \emptyset\right\} \\
& =\{\beta \in \mathrm{B} \mid \beta \cap f(\mathrm{X}) \neq \emptyset\} .
\end{aligned}
$$

Remarquons que $\varphi(\mathrm{X})$ est connexe si c'est le cas de X. Remarquons également que pour toute famille $\left(\mathrm{X}_{i}\right)_{i \in \mathrm{I}}$ de parties de $\mathrm{B}$, on a :

$$
\varphi\left(\bigcup_{i \in \mathrm{I}} \mathrm{X}_{i}\right)=\bigcup_{i \in \mathrm{I}} \varphi\left(\mathrm{X}_{i}\right), \quad \varphi\left(\bigcap_{i \in \mathrm{I}} \mathrm{X}_{i}\right) \subset \bigcap_{i \in \mathrm{I}} \varphi\left(\mathrm{X}_{i}\right) .
$$

On appellera attracteur toute partie $\mathrm{X} \subset \mathrm{B}$ vérifiant $\varphi(\mathrm{X}) \subset \mathrm{X}$, ce qui est équivalent dans $\mathrm{M}$ à l'inclusion $f(\mathrm{X}) \subset \operatorname{Int}(\mathrm{X})$. La réunion et l'intersection d'une famille d'attracteurs est encore un attracteur.

On définit de façon analogue une application

$$
\varphi_{-}: \mathscr{P}(\mathrm{B}) \rightarrow \mathscr{P}(\mathrm{B})
$$

en posant :

$$
\begin{aligned}
\varphi_{-}(\mathrm{X}) & =\left\{\beta \in \mathrm{B} \mid \text { il existe } \beta^{\prime} \in \mathrm{X} \text { tel que } \beta \cap f^{-1}\left(\beta^{\prime}\right) \neq \emptyset\right\} \\
& =\left\{\beta \in \mathrm{B} \mid \beta \cap f^{-1}(\mathrm{X}) \neq \emptyset\right\} .
\end{aligned}
$$


Les relations

$$
\beta^{\prime} \in \varphi^{n}(\{\beta\}), \quad \beta \in \varphi_{-}^{n}\left(\left\{\beta^{\prime}\right\}\right)
$$

sont équivalentes : elles signifient qu'il existe une suite $\left(\beta_{i}\right)_{0 \leq i \leq n}$ de briques, avec $\beta_{0}=\beta$ et $\beta_{n}=\beta^{\prime}$, telle que $f\left(\beta_{i}\right) \cap \beta_{i+1} \neq \emptyset$ si $i \in\{0, \ldots, n-1\}$. Un répulseur est, par définition, une partie positivement invariante par $\varphi_{-}$. Remarquons que $\mathrm{X} \subset \mathrm{B}$ est un répulseur si et seulement si $\mathrm{B} \backslash \mathrm{X}$ est un attracteur.

\section{Décompositions libres}

Soit $f$ un homéomorphisme de $\mathrm{M}$ sans point fixe. On dira qu'une décomposition en briques $\mathscr{D}=(\mathrm{S}, \mathrm{A}, \mathrm{B})$ est libre si toute brique de $\mathscr{D}$ est libre, c'est-à-dire disjointe de son image par $f$. Il est toujours possible, quitte à choisir les briques assez petites, de construire une décomposition libre. On peut alors parler naturellement de partie libre $\mathrm{X} \subset \mathrm{B}$ si $f(\mathrm{X}) \cap \mathrm{X}=\emptyset$. Énonçons le résultat fondamental sur lequel est basée toute notre étude :

Proposition 2.1. - Soit $f: \mathbf{R}^{2} \rightarrow \mathbf{R}^{2}$ un homéomorphisme de Brouwer. Si $\mathscr{D}=$ (S, A, B) est une décomposition libre de $\mathbf{R}^{2}$, alors pour toute brique $\beta \in \mathrm{B}$, l'attracteur $\bigcup_{n \geq 1} \varphi^{n}(\{\beta\})$ ne contient pas $\beta$.

Le lemme de Franks [Fr1], énoncé sous sa forme usuelle, nous dit qu'il n'existe pas de suite $\left(\beta_{i}\right)_{i \in \mathbf{Z} / n \mathbf{Z}}$ de briques ouvertes, telle que $f\left(\beta_{i}\right) \cap \beta_{i+1} \neq \emptyset$ pour tout $i \in \mathbf{Z} / n \mathbf{Z}$. La proposition 2.1, qui est due à Guillou et Le Roux (voir [LeR] p. 39), exprime la validité du résultat pour les briques fermées. On a plus généralement :

Corollaire 2.2. - Soit $f: \mathbf{R}^{2} \rightarrow \mathbf{R}^{2}$ un homéomorphisme de Brouwer. Si $\mathscr{D}=$

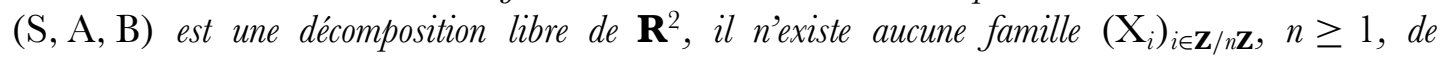
parties libres et connexes de $\mathrm{B}$, disjointes deux à deux, telles que

$$
\mathrm{X}_{i+1} \bigcap\left(\bigcup_{n \geq 1} \varphi^{n}\left(\mathrm{X}_{i}\right)\right) \neq \emptyset
$$

pour tout $i \in \mathbf{Z} / n \mathbf{Z}$.

Démonstration. - On considère la partition de $\mathrm{B}$ formée des $\mathrm{X}_{i}$ et des briques n'appartenant à aucune partie $\mathrm{X}_{i}$. La sous-décomposition de $\mathscr{D}$ définie par cette partition est libre et on applique alors la proposition 2.1. 
L'article [LeC5] est basé sur la notion de décomposition libre maximale, notion introduite par Sauzet dans sa thèse. Soit $f$ un homéomorphisme sans point fixe d'une surface $\mathrm{M}$. On dit que $\mathscr{D}$ est une décomposition libre maximale si $\mathscr{D}$ est libre et si aucune sous-décomposition stricte de $\mathscr{D}$ n'est libre. En particulier la décomposition $\mathscr{D}$ est remplie. Une application simple du lemme de Zorn nous dit que si $\mathscr{D}$ est une décomposition libre de M, il existe une sous-décomposition $\mathscr{D}^{\prime}$ de $\mathscr{D}$ qui est libre maximale.

Soit $f: \mathbf{R}^{2} \rightarrow \mathbf{R}^{2}$ un homéomorphisme de Brouwer et $\mathscr{D}=(\mathrm{S}, \mathrm{A}, \mathrm{B})$ une décomposition libre maximale du plan. Toute arête $\alpha \in \mathrm{A}$ est adjacente à deux briques dont la réunion n'est pas libre. L'image par $f$ de l'une de ces briques rencontre donc la seconde, mais l'image de cette seconde brique ne rencontre pas la première, d'après la proposition 2.1. Il existe donc une orientation naturelle du squelette de $\mathscr{D}$ : pour toute arête $\alpha$ l'image de la brique adjacente à gauche de $\alpha$ rencontre la brique adjacente à droite de $\alpha$.

\section{Décompositions à relèvement libre}

Nous allons voir maintenant une notion plus faible de maximalité, due également à Sauzet, et nous verrons comment orienter le squelette sous cette condition.

Considérons un revêtement galoisien $\pi: \mathrm{M} \rightarrow \widetilde{\mathrm{M}}$ entre deux surfaces et notons $\mathrm{G}$ le groupe des automorphismes du revêtement. On se donne un homéomorphisme $f$ de $\mathrm{M}$ sans point fixe et commutant avec tous les éléments de $\mathrm{G}$ et on note $\widetilde{f}$ l'homéomorphisme de $\widetilde{M}$ relevé par $f$. On dit que $\widetilde{\mathscr{D}}$ est une décomposition à relèvement libre maximale de $\tilde{\mathrm{M}}$, si $\mathscr{D}=\pi^{-1}(\widetilde{\mathscr{D}})$ est libre et si aucune sousdécomposition stricte de $\widetilde{\mathscr{D}}$ ne vérifie cette propriété. On dit alors que $\mathscr{D}$ est une décomposition libre équivariante maximale. La sous-décomposition de $\mathscr{D}=(\mathrm{S}, \mathrm{A}, \mathrm{B})$ obtenue à partir de la partition triviale de $\mathrm{B}$ est libre et équivariante, on en déduit que $\mathscr{D}$ est remplie. Ce n'est pas nécessairement le cas de $\widetilde{\mathscr{D}}$, on peut avoir pour cette décomposition des arêtes adjacentes à une seule brique et des arêtes qui sont des cercles avec une extrémité. Là encore, on montre que si $\mathscr{D}$ est une décomposition libre équivariante de $\mathrm{M}$, il existe une sous-décomposition $\mathscr{D}^{\prime}$ de $\mathscr{D}$ qui est libre équivariante maximale. Il faut noter qu'une décomposition libre équivariante maximale n'est pas nécessairement une décomposition libre maximale (au sens du paragraphe précédent). Considérons le groupe $\mathrm{G}$ formé des itérés de la translation du plan

$$
\mathrm{T}:(x, y) \mapsto(x+1, y) .
$$

Sauzet montre dans sa thèse qu'il n'existe aucune décomposition libre maximale de $\mathbf{R}^{2}$ qui soit G-équivariante pour l'homéomorphisme de Brouwer $f=\mathrm{T}^{k}$, si $k \geq 2$.

Remarquons que si $\mathscr{D}$ est une décomposition de $\mathrm{M}$ qui est G-équivariante, alors les ensembles $\mathrm{S}$, A et $\mathrm{B}$ sont invariants sous l'action naturelle de $\mathrm{G}$ et les 
applications $\varphi$ et $\varphi_{-}$sont équivariantes : pour toute partie $\mathrm{X} \subset \mathrm{B}$ et pour tout $\mathrm{T} \in \mathrm{G}$, on a

$$
\varphi(\mathrm{TX})=\mathrm{T} \varphi(\mathrm{X}), \quad \varphi_{-}(\mathrm{TX})=\mathrm{T} \varphi_{-}(\mathrm{X}) .
$$

Dans le cas où $\mathscr{D}$ est une décomposition libre équivariante maximale, on a le résultat évident suivant :

Lemme 2.3. - Si $\left(\mathrm{X}_{i}\right)_{i \in \mathrm{I}}$ est une partition de $\mathrm{B}$ en parties connexes, invariante sous l'action naturelle de $\mathrm{G}$, et qui n'est pas la partition triviale, alors l'une au moins des parties $\mathrm{X}_{i}$ n'est pas libre.

Démonstration. - L'ensemble $\Sigma^{\prime}=\bigcup_{i \in \mathrm{I}} \partial \mathrm{X}_{i}$ est le squelette d'une sous-décomposition stricte de $\mathscr{D}$ équivariante dont les briques sont les $\mathrm{X}_{i}$. Cette décomposition n'est pas libre par maximalité de $\mathscr{D}$.

\section{Étude des briques d'une décomposition libre équivariante maximale du plan}

On se place dorénavant et jusqu'à la fin de la partie I de cet article dans les hypothèses du théorème 1.2. On se donne un homéomorphisme de Brouwer $f$ commutant avec les éléments d'un groupe $\mathrm{G}$ d'homéomorphismes du plan conservant l'orientation, qui agit proprement et librement sur $\mathbf{R}^{2}$. On se donne une décomposition libre équivariante maximale $\mathscr{D}=(\mathrm{S}, \mathrm{A}, \mathrm{B})$, on note $\widetilde{\mathscr{D}}$ la décomposition de $\mathrm{M}=\mathbf{R}^{2} / \mathrm{G}$ relevée par $\mathscr{D}$ et on note $\pi: \mathbf{R}^{2} \rightarrow \mathrm{M}$ le revêtement universel de $\mathbf{M}$. On va commencer par étudier la structure des briques.

La frontière d'une brique $\beta \in \mathrm{B}$ est une sous-variété de dimension un, dont les composantes connexes, appelées arcs frontières, sont des $\mathscr{D}$-droites ou des $\mathscr{D}$-cercles. Chaque arc frontière de $\beta$ est l'unique arc frontière d'une composante connexe de $\mathrm{B} \backslash\{\beta\}$, on obtient ainsi une bijection naturelle entre les arcs frontières de $\beta$ et les composantes connexes de $\mathrm{B} \backslash\{\beta\}$. Les ensembles $\varphi(\{\beta\})$ et $\varphi_{-}(\{\beta\})$ sont connexes et ne contiennent pas $\beta$, puisque $\beta$ est libre. Il existe donc deux composantes connexes $\mathrm{X}_{\beta}^{+}$et $\mathrm{X}_{\beta}^{-}$de $\mathrm{B} \backslash\{\beta\}$, éventuellement égales, telles que

$$
\varphi(\{\beta\}) \subset \mathrm{X}_{\beta}^{+}, \quad \varphi_{-}(\{\beta\}) \subset \mathrm{X}_{\beta}^{-},
$$

et on a bien évidemment, pour $\mathrm{T} \in \mathrm{G}$,

$$
\mathrm{TX}_{\beta}^{+}=\mathrm{X}_{\mathrm{T} \beta}^{+}, \quad \mathrm{TX}_{\beta}^{-}=\mathrm{X}_{\mathrm{T} \beta}^{-} .
$$

Dans le cas où $\mathrm{X}_{\beta}^{+}=\mathrm{X}_{\beta}^{-}$, on écrira

$$
\mathrm{X}_{\beta}^{+}=\mathrm{X}_{\beta}^{-}=\mathrm{X}_{\beta}^{ \pm} \text {. }
$$


L'arc $\partial \mathrm{X}_{\beta}^{+}$est invariant sous l'action du stabilisateur de $\beta$ :

$$
\mathrm{G}_{\beta}=\{\mathrm{T} \in \mathrm{G} \mid \mathrm{T} \beta=\beta\} .
$$

Ce sous-groupe agit sur l'arc, proprement, librement et en préservant l'orientation, puisque $\mathrm{X}_{\beta}^{+}$est $\mathrm{G}_{\beta}$-invariant et puisque chaque élément $\mathrm{T} \in \mathrm{G}$ préserve l'orientation de $\mathbf{R}^{2}$. Si cet arc est un cercle, le stabilisateur est trivial ; si c'est une droite, il est trivial ou alors infini cyclique : il existe $\mathrm{T} \in \mathrm{G}^{*}$ tel que $\mathrm{G}_{\beta}=\left\{\mathrm{T}^{k}, k \in \mathbf{Z}\right\}$.

On va préciser la structure des briques de $\mathscr{D}$.

Lemme 2.4. - On a $\mathrm{B} \backslash\left(\mathrm{X}_{\beta}^{+} \cup \mathrm{X}_{\beta}^{-}\right)=\{\beta\}$.

Démonstration. - La partie

$$
\mathrm{X}=\mathrm{B} \backslash\left(\mathrm{X}_{\beta}^{+} \cup \mathrm{X}_{\beta}^{-}\right)
$$

est connexe comme réunion de $\{\beta\}$ et de composantes connexes de $\mathrm{B} \backslash\{\beta\}$. Elle est également libre. En effet, la partie $f(\mathrm{X})$ est connexe, elle est disjointe de $\partial \mathrm{X}_{\beta}^{+}$, puisque

$$
f^{-1}\left(\partial \mathrm{X}_{\beta}^{+}\right) \subset f^{-1}(\beta) \subset \operatorname{Int}\left(\mathrm{X}_{\beta}^{-}\right),
$$

et elle rencontre $\mathrm{X}_{\beta}^{+}$puisque $\beta \in \mathrm{X}$. Elle est donc contenue dans $\operatorname{Int}\left(\mathrm{X}_{\beta}^{+}\right)$et disjointe de $\mathrm{X}$. On montre de même que la partie $f^{-1}(\mathrm{X})$ est contenue dans $\operatorname{Int}\left(\mathrm{X}_{\beta}^{-}\right)$. Plus précisément, on a

$$
\varphi(\mathrm{X}) \subset \mathrm{X}_{\beta}^{+}, \quad \varphi_{-}(\mathrm{X}) \subset \mathrm{X}_{\beta}^{-} .
$$

Pour des raisons analogues, on a

$$
\varphi\left(\mathrm{X}_{\beta}^{+}\right) \subset \mathrm{X}_{\beta}^{+}, \quad \varphi_{-}\left(\mathrm{X}_{\beta}^{-}\right) \subset \mathrm{X}_{\beta}^{-} .
$$

Pour montrer que $\mathrm{X}=\{\beta\}$, il faut montrer que $\mathrm{T}(\mathrm{X})$ et $\mathrm{X}$ sont disjoints dans $\mathrm{B}$ (mais pas nécessairement dans $\mathbf{R}^{2}$ ) si $\mathrm{T} \notin \mathrm{G}_{\beta}$. En effet, les briques $\mathrm{T}(\mathrm{X}), \mathrm{T} \in \mathrm{G}$, seront alors disjointes ou égales. Le lemme 2.3 appliqué à la partition de $\mathrm{B}$ formée des translatés de $\mathrm{X}$ et de toutes les briques restantes impliquera que $\mathrm{X}=\{\beta\}$.

Fixons $\mathrm{T} \notin \mathrm{G}_{\beta}$. Montrons d'abord par l'absurde que $\beta \notin \mathrm{TX} \cap \mathrm{T}^{-1} \mathrm{X}$. Aucun des ensembles

$$
\mathrm{TX}_{\beta}^{+}, \quad \mathrm{TX}_{\beta}^{-}, \quad \mathrm{T}^{-1} \mathrm{X}_{\beta}^{+}, \quad \mathrm{T}^{-1} \mathrm{X}_{\beta}^{+}
$$

n'est libre. Si $\beta \in \mathrm{TX} \cap \mathrm{T}^{-1} \mathrm{X}$, alors chacun des quatres ensembles est inclus dans une composante connexe de $\mathrm{B} \backslash\{\beta\}$ et n'est pas inclus dans $\mathrm{X}$ puisque $\mathrm{X}$ est libre. Ces ensembles sont donc contenus dans $\mathrm{X}_{\beta}^{+} \cup \mathrm{X}_{\beta}^{-}$. On en déduit

$$
\mathrm{T}\left(\mathrm{X}_{\beta}^{+} \cup \mathrm{X}_{\beta}^{-}\right)=\mathrm{X}_{\beta}^{+} \cup \mathrm{X}_{\beta}^{-},
$$

ce qui est impossible puisque, $\mathrm{T}$ n'appartenant pas à $\mathrm{G}_{\beta}$, on a $\mathrm{T}(\mathrm{X}) \neq \mathrm{X}$. 
On peut donc supposer, quitte à changer $\mathrm{T}$ en $\mathrm{T}^{-1}$, que $\beta \notin \mathrm{TX}$. On va montrer que TX est inclus dans $\mathrm{X}_{\beta}^{+}$ou dans $\mathrm{X}_{\beta}^{-}$et est donc disjoint de $\mathrm{X}$. Puisque $\beta \notin \mathrm{TX}$, on sait que TX est dans une composante connexe de $\mathrm{B} \backslash\{\beta\}$. Si cette composante n'est ni $\mathrm{X}_{\beta}^{+}$ni $\mathrm{X}_{\beta}^{-}$, on a $\mathrm{TX} \subset \mathrm{X}$ et même $\mathrm{TX} \subset \mathrm{X} \backslash\{\beta\}$. En particulier $\partial \mathrm{TX} \cap \partial \mathrm{X}=\emptyset$.

On a le résultat élémentaire suivant :

Lemme 2.5. - Soit $\mathscr{D}=(\mathrm{S}, \mathrm{A}, \mathrm{B})$ une décomposition en briques d'une surface $\mathrm{M}$. Si $\left(\mathrm{X}_{k}\right)_{k \geq 0}$ est une suite strictement croissante de parties de $\mathrm{B}$ et si $\partial \mathrm{X}_{k} \cap \partial \mathrm{X}_{k+1}=\emptyset$, pour tout $k \geq 0$, alors $\bigcup_{k \geq 0} \mathrm{X}_{k}=\mathrm{B}$.

$\mathrm{M}$ est connexe.

Démonstration. - Il suffit de remarquer que $\partial\left(\bigcup_{k \geq 0} \mathrm{X}_{k}\right)=\emptyset$ et de rappeler que
$\square$

On peut appliquer le lemme à la suite $\left(\mathrm{T}^{-k} \mathrm{X}\right)_{k \geq 0}$ et en déduire qu'il existe $k \geq 0$ tel que $\varphi(\mathrm{X}) \cap \mathrm{T}^{-k} \mathrm{X} \neq \emptyset$. Ceci est impossible. En effet, on sait d'une part que $\mathrm{X} \subset \mathrm{T}^{-k}(\mathrm{X})$ et d'autre part que $\mathrm{T}^{-k}(\mathrm{X})$ est libre puisque c'est le cas de $\mathrm{X}$, on en déduit :

$$
\varphi(\mathrm{X}) \cap \mathrm{T}^{-k} \mathrm{X} \subset \varphi\left(\mathrm{T}^{-k} \mathrm{X}\right) \cap \mathrm{T}^{-k} \mathrm{X}=\emptyset .
$$

On peut maintenant expliquer la structure des briques :

Proposition 2.6. - Il y a trois types de briques possibles dans $\mathrm{B}$.

i) Les briques $\beta$ qui sont des disques fermés. Leur stabilisateur est trivial. On a $\mathrm{B} \backslash\{\beta\}=\mathrm{X}_{\beta}^{ \pm}$et on sait que $\partial \beta=\partial \mathrm{X}_{\beta}^{ \pm}$est un cercle.

ii) Les briques $\beta$ qui sont des demi-plans fermés. Leur stabilisateur est trivial. On a $\mathrm{B} \backslash\{\beta\}=\mathrm{X}_{\beta}^{ \pm}$et on sait que $\partial \beta=\partial \mathrm{X}_{\beta}^{ \pm}$est une droite.

iii) Les briques $\beta$ qui sont des bandes fermées. Leur stabilisateur est trivial ou alors infini cyclique. On a $\mathrm{B} \backslash\{\beta\}=\mathrm{X}_{\beta}^{+} \sqcup \mathrm{X}_{\beta}^{-}$et on sait que $\partial \beta$ est formé des deux droites disjointes $\partial \mathrm{X}_{\beta}^{+}$et $\partial \mathrm{X}_{\beta}^{-}$.

Démonstration. - Si $\partial \mathrm{X}_{\beta}^{+}$est un cercle, le classique théorème du point fixe de Brouwer nous dit que $\mathrm{X}_{\beta}^{+}$, qui contient $f\left(\partial \mathrm{X}_{\beta}^{+}\right)$puisque $f\left(\partial \mathrm{X}_{\beta}^{+}\right) \subset f(\mathrm{X}) \subset \mathrm{X}_{\beta}^{+}$, est l'anneau et non pas le disque bordé par $\partial \mathrm{X}_{\beta}^{+}$. Ainsi $\beta$ est borné et $\partial \mathrm{X}_{\beta}^{-}$est donc un cercle. La composante $\mathrm{X}_{\beta}^{-}$est également un anneau non borné et coïncide donc avec $\mathrm{X}_{\beta}^{+}$. Le lemme 2.4 nous dit que $\beta$ est un disque. Son stabilisateur est évidemment trivial.

Il reste à étudier le cas où les $\operatorname{arcs} \partial \mathrm{X}_{\beta}^{+}$et $\partial \mathrm{X}_{\beta}^{-}$sont des droites. Le lemme 2.4 nous dit que $\beta$ est un demi-plan si $\partial \mathrm{X}_{\beta}^{+}=\partial \mathrm{X}_{\beta}^{-}$, une bande si $\partial \mathrm{X}_{\beta}^{+} \neq \partial \mathrm{X}_{\beta}^{-}$. 
Pour montrer la proposition, il reste à vérifier que le stabilisateur d'un demiplan $\beta \in \mathrm{B}$ est trivial. On peut construire un segment $\gamma$ joignant un point $z \in$ $\partial \beta=\partial \mathrm{X}_{\beta}^{ \pm}$à $f(z)$ et disjoint sauf aux extrémités de $\beta$ et de $f(\beta)$. Si le stabilisateur de $\beta$ contient un élément non trivial $\mathrm{T}$, alors $\mathrm{T}$ préserve l'orientation sur $\partial \beta$. Puisque $\beta$ est libre, T pousse les points de $\partial \beta$ et les points de $\partial f(\beta)$ en des sens opposés. Ainsi le segment $\mathrm{T}^{k}(\gamma)$, qui joint $\mathrm{T}^{k}(z)$ à $f\left(\mathrm{~T}^{k}(z)\right)$, doit rencontrer $\gamma$. Ceci est impossible dès que $k$ est suffisamment grand puisque l'action de $\mathrm{G}$ sur $\mathbf{R}^{2}$ est propre et libre.

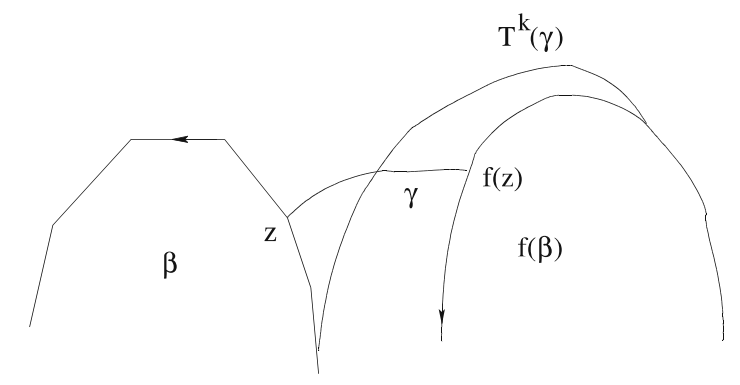

Étude des briques adjacentes

Nous allons continuer notre étude des briques de B par le résultat suivant :

Proposition 2.7. - Soit $\alpha \in \mathrm{A}$ une arête et $\beta, \beta^{\prime}$ les briques adjacentes à $\alpha$. Si les briques $\pi(\beta), \pi\left(\beta^{\prime}\right)$ de $\widetilde{\mathrm{B}}$ sont distinctes, alors $\beta \cup \beta^{\prime}$ n'est pas libre. Si $\beta^{\prime}=\mathrm{T}_{0}(\beta)$, où $\mathrm{T}_{0} \in \mathrm{G}^{*}$, alors $\bigcup_{k \in \mathbf{Z}} \mathrm{T}_{0}^{k}(\beta)$ n'est pas libre.

Supposons dans un premier temps que l'arête $\widetilde{\alpha}=\pi(\alpha) \in \widetilde{\mathrm{A}}$ ne soit pas une arête compacte à une extrémité. L'ensemble $\Sigma(\widetilde{\mathscr{D}}) \backslash \operatorname{int}(\widetilde{\alpha})$ est alors le squelette d'une décomposition en briques $\widetilde{\mathscr{D}}^{\prime}$. Le squelette de la décomposition relevée $\mathscr{D}^{\prime}=$ $\left(\mathrm{S}^{\prime}, \mathrm{A}^{\prime}, \mathrm{B}^{\prime}\right)$ est

$$
\Sigma\left(\mathscr{D}^{\prime}\right)=\Sigma(\mathscr{D}) \backslash \bigcup_{\mathrm{T} \in \mathrm{G}} \operatorname{int}(\mathrm{T} \alpha),
$$

et on sait que cette décomposition n'est pas libre. Toute brique $\beta^{\prime \prime} \in \mathrm{B}$ qui n'est pas dans la G-orbite de $\beta$, ni dans celle de $\beta^{\prime}$, est une brique de $\mathscr{D}^{\prime}$. On notera $\mathrm{B}^{\prime \prime} \subset \mathrm{B}^{\prime}$ l'ensemble de telles briques.

Si $\pi(\beta) \neq \pi\left(\beta^{\prime}\right)$ et si $\beta$ et $\beta^{\prime}$ ont toutes deux un stabilisateur trivial, alors ni $\beta$, ni $\beta^{\prime}$ ne contiennent de translatés $\mathrm{T} \alpha, \mathrm{T} \in \mathrm{G}^{*}$. L'ensemble $\mathrm{B}^{\prime}$ est formé des briques de $\mathrm{B}^{\prime \prime}$ et des translatés de $\beta \cup \beta^{\prime}$. On en déduit que $\beta \cup \beta^{\prime}$ n'est pas libre, grâce au lemme 2.3.

Si $\pi(\beta) \neq \pi\left(\beta^{\prime}\right)$, si $\mathrm{G}_{\beta^{\prime}}$ est trivial et si $\mathrm{G}_{\beta}$ ne l'est pas, alors $\beta^{\prime}$ ne contient aucun translaté non trivial de $\alpha$ et les seuls translatés de $\alpha$ contenus dans $\beta$ 
sont les translatés $\mathrm{T} \alpha, \mathrm{T} \in \mathrm{G}_{\beta}$. L'ensemble $\mathrm{B}^{\prime}$ est formé des briques de $\mathrm{B}^{\prime \prime}$ et des translatés de l'ensemble $\beta \bigcup_{\mathrm{T} \in \mathrm{G}_{\beta}} \mathrm{T} \beta^{\prime}$. On sait donc que cet ensemble n'est pas libre. Il reste à montrer que ce n'est pas le cas non plus de $\beta \cup \beta^{\prime}$. Pour cela on raisonne par l'absurde. On sait, d'après la proposition 2.6 que $\mathrm{B} \backslash\{\beta\}$ a deux composantes connexes $\mathrm{X}_{\beta}^{+}$et $\mathrm{X}_{\beta}^{-}$invariantes sous l'action de $\mathrm{G}_{\beta}$. On peut supposer, sans perte de généralités que $\beta^{\prime} \in \mathrm{X}_{\beta}^{-}$et donc que $\mathrm{T} \beta^{\prime} \in \mathrm{X}_{\beta}^{-}$, pour tout $\mathrm{T} \in \mathrm{G}_{\beta}$. Si $f\left(\beta^{\prime}\right) \cap \beta=\emptyset$, alors $f\left(\mathrm{~T} \beta^{\prime}\right) \cap \beta=\emptyset$, pour tout $\mathrm{T} \in \mathrm{G}_{\beta}$. L'ensemble connexe $\varphi\left(\beta \bigcup_{\mathrm{T} \in \mathrm{G}_{\beta}} \mathrm{T} \beta^{\prime}\right)$ est inclus dans une composante connexe de $\mathrm{B} \backslash\{\beta\}$, à savoir dans $\mathrm{X}_{\beta}^{+}$ puisque $\varphi(\beta) \subset \mathrm{X}_{\beta}^{+}$. On en déduit que $\beta \bigcup_{\mathrm{T} \in \mathrm{G}_{\beta}} \mathrm{T} \beta^{\prime}$ est libre, ce qui est impossible.

Si $\pi(\beta) \neq \pi\left(\beta^{\prime}\right)$ et si $\beta$ et $\beta^{\prime}$ ont toutes deux le même stabilisateur non trivial, alors $\mathrm{B}^{\prime}$ est formé des briques de $\mathrm{B}^{\prime \prime}$ et des translatés $\mathrm{T}\left(\beta \cup \beta^{\prime}\right), \mathrm{T} \in \mathrm{G}$. On en déduit que $\beta \cup \beta^{\prime}$ n'est pas libre.

Si $\pi(\beta) \neq \pi\left(\beta^{\prime}\right)$ et si les stabilisateurs $\mathrm{G}_{\beta}$ et $\mathrm{G}_{\beta^{\prime}}$ sont distincts et non triviaux, on montre là encore que $\beta \cup \beta^{\prime}$ n'est pas libre mais pour une autre raison. On peut supposer qu'il existe $\mathrm{T}^{\prime} \in \mathrm{G}_{\beta^{\prime}}$ tel que $\mathrm{T}^{\prime} \beta \neq \beta$. On sait, d'après la proposition 2.6, que $\mathrm{B} \backslash\{\beta\}$ a deux composantes connexes $\mathrm{X}_{\beta}^{+}$et $\mathrm{X}_{\beta}^{-}$et on peut supposer que $\beta^{\prime} \in \mathrm{X}_{\beta}^{-}$(et donc que $\beta^{\prime} \in \mathrm{X}_{\mathrm{T}^{\prime} \beta}^{-}$). Les deux briques $\beta^{\prime}$ et $\mathrm{T}^{\prime} \beta$, étant adjacentes, appartiennent à la même composante connexe de $\mathrm{B} \backslash\{\beta\}$, à savoir à $\mathrm{X}_{\beta}^{-}$. La partie $\mathrm{X}_{\beta}^{+} \cup\left\{\beta, \beta^{\prime}\right\}$, qui est connexe et ne contient pas $\mathrm{T}^{\prime} \beta$ est contenue dans une composante connexe de $\mathrm{B} \backslash\left\{\mathrm{T}^{\prime} \beta\right\}$, à savoir dans $\mathrm{X}_{\mathrm{T}^{\prime} \beta}^{-}$, puisque $\beta^{\prime} \in \mathrm{X}_{\mathrm{T}^{\prime} \beta}^{-}$. On en déduit que $\mathrm{X}_{\beta}^{+}$et $\mathrm{X}_{\mathrm{T}^{\prime} \beta}^{+}$sont disjoints dans $\mathrm{B}$. Montrons par l'absurde que $f\left(\beta^{\prime}\right) \cap \beta \neq \varnothing$. Si ce n'est pas le cas, $\varphi\left(\left\{\beta, \beta^{\prime}\right\}\right)$ est dans une composante connexe de $\mathrm{B} \backslash\{\beta\}$, à savoir dans $\mathrm{X}_{\beta}^{+}$. Ainsi, on a $\varphi\left(\beta^{\prime}\right) \subset \mathrm{X}_{\beta}^{+}$. De même $\varphi\left(\beta^{\prime}\right)=\varphi\left(\mathrm{T}^{\prime} \beta^{\prime}\right) \subset \mathrm{X}_{\mathrm{T}^{\prime} \beta}^{+}$, ce qui impossible puisque $\mathrm{X}_{\beta}^{+}$et $\mathrm{X}_{\mathrm{T}^{\prime} \beta}^{+}$sont disjoints.

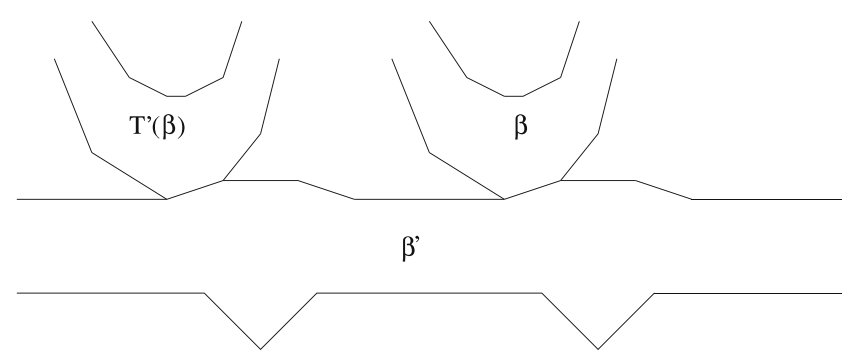

Si $\beta^{\prime}=\mathrm{T}_{0} \beta$, où $\mathrm{T}_{0} \in \mathrm{G}^{*}$, et si le stabilisateur de $\beta$ est trivial, la seule arête translatée $\mathrm{T} \alpha, \mathrm{T} \in \mathrm{G}^{*}$, contenue dans $\beta$ est $\mathrm{T}_{0}^{-1} \alpha$. L'ensemble $\mathrm{B}^{\prime}$ est formé des briques de $\mathrm{B}^{\prime \prime}$ et des translatés de $\bigcup_{k \in \mathbf{Z}} \mathrm{T}_{0}^{k} \beta$. Ce dernier ensemble n'est pas libre.

Si $\beta^{\prime}=\mathrm{T}_{0} \beta$, où $\mathrm{T}_{0} \in \mathrm{G}^{*}$, et si le stabilisateur de $\beta$ est non trivial, alors la frontière de $\beta$ est formée de deux arcs $\partial \mathrm{X}_{\beta}^{+}$et $\partial \mathrm{X}_{\beta}^{-}$, d'après la proposition 2.6. On 
peut supposer que $\alpha \subset \partial \mathrm{X}_{\beta}^{-}$. Les seuls translatés de $\alpha$ contenus dans la frontière de $\beta$ sont les arêtes $\mathrm{T} \alpha, \mathrm{T} \in \mathrm{G}_{\beta}$, qui sont sur $\partial \mathrm{X}_{\beta}^{-}$et les arêtes $\mathrm{TT}_{0}^{-1} \alpha, \mathrm{T} \in \mathrm{G}_{\beta}$ qui sont sur $\partial \mathrm{X}_{\beta}^{+}$. L'image par $\pi$ de l'intérieur de $\beta$ est un anneau ouvert de $\mathrm{M}=\mathbf{R}^{2} / \mathrm{G}$. Choisissons un cercle essentiel $\tilde{\gamma}_{1}$ dans cet anneau. La composante connexe de la préimage de ce cercle qui est dans $\beta$ est une droite $\Gamma_{1}$ qui s'écrit $\Gamma_{1}=\prod_{k \in \mathbf{Z}} \mathrm{T}^{k}\left(\gamma_{1}\right)$, où $\gamma_{1}$ est un segment joignant un point $z_{1}$ à $\mathrm{T}\left(z_{1}\right)$. Considérons un segment $\gamma_{0}$ joignant un point $z_{0} \in \mathrm{T}_{0}^{-1}(\alpha)$ à $\mathrm{T}_{0}\left(z_{0}\right) \in \alpha$, à valeurs dans $\operatorname{Int}(\beta)$ sauf aux extrémités. Cet arc s'envoie par $\pi$ sur un lacet $\widetilde{\gamma}_{0}$ de $\mathrm{M}$ dont l'intersection algébrique avec $\tilde{\gamma}_{1}$ est \pm 1 . On peut d'ailleurs choisir $\gamma_{0}$ pour que $\widetilde{\gamma}_{0}$ et $\widetilde{\gamma}_{1}$ ne se rencontrent qu'en un point. Les droites $\Gamma_{0}=\prod_{k \in \mathbf{Z}} \mathrm{T}_{0}^{k}\left(\gamma_{0}\right)$ et $\Gamma_{1}$ n'ont alors qu'un point d'intersection $z_{2}$ et s'intersectent transversalement. Montrons maintenant que $f\left(\Gamma_{1}\right)$ rencontre $\Gamma_{0}$ et donc que $f(\beta)$ rencontre $\bigcup_{k \in \mathbf{Z}} \mathrm{T}_{0}^{k}(\beta)$. Pour cela raisonnons par l'absurde et supposons que $f\left(\Gamma_{1}\right)$ soit dans une composante connexe de $\mathbf{R}^{2} \backslash \Gamma_{0}$. Quitte à changer $\mathrm{T}$ en $\mathrm{T}^{-1}$, on peut supposer que les $\operatorname{arcs} \mathrm{T}^{k}\left(\gamma_{1}\right)$ sont dans l'autre composante connexe pour $k$ assez grand $\left(k \geq k_{0}\right)$. Choisissons un arc $\gamma$ joignant $z_{1}$ à $f\left(z_{1}\right)$. Pour tout $k \geq k_{0}$, l'arc $\mathrm{T}^{k}(\gamma)$ joint $\mathrm{T}^{k}\left(z_{1}\right)$ à $\mathrm{T}^{k}\left(f\left(z_{1}\right)\right)=f\left(\mathrm{~T}^{k}\left(z_{1}\right)\right)$ et doit donc rencontrer $\Gamma_{0}$ : il existe $l_{k} \in \mathbf{Z}$ tel que $\mathrm{T}^{k}(\gamma) \cap \mathrm{T}_{0}^{l_{k}}\left(\gamma_{0}\right) \neq \emptyset$. Ainsi on a $\gamma \cap \mathrm{T}^{-k} \circ \mathrm{T}_{0}^{l_{k}}\left(\gamma_{0}\right) \neq \emptyset$. Puisque l'action de $\mathrm{G}$ est propre est libre, on peut donc trouver $k^{\prime}>k \geq k_{0}$ tel que $\mathrm{T}^{-k} \circ \mathrm{T}_{0}^{l_{k}}=\mathrm{T}^{-k^{\prime}} \circ \mathrm{T}_{0}^{l_{k^{\prime}}}$ et donc $\mathrm{T}^{k^{\prime}-k}=\mathrm{T}_{0}^{l_{k^{\prime}}-l_{k}}$. Ceci est bien sûr impossible puisque $\mathrm{T}^{k^{\prime}-k}\left(z_{2}\right) \neq \mathrm{T}_{0}^{l^{\prime}-l_{k}}\left(z_{2}\right)$.

Supposons maintenant que $\widetilde{\alpha}=\pi(\alpha)$ soit une arête compacte ayant une unique extrémité $\widetilde{\sigma} \in \widetilde{\mathrm{S}}$ et notons $\widetilde{\alpha}^{\prime} \in \widetilde{\mathrm{A}}$ la seconde arête dont $\widetilde{\sigma}$ est une extrémité. L'arête $\alpha$ est alors un segment et la composante connexe de $\pi^{-1}(\widetilde{\alpha})$ qui contient $\alpha$ s'écrit $\Gamma=\bigcup_{k \in \mathbf{Z}} \mathrm{T}^{k} \alpha$, où $\mathrm{T} \in \mathrm{G}^{*}$. On supposera que $\beta$ est la brique, de stabilisateur $\mathrm{G}_{\beta}=\left\{\mathrm{T}^{k} \mid k \in \mathbf{Z}\right\}$, dont $\Gamma$ est un arc frontière et on supposera que $\Gamma=\partial \mathrm{X}_{\beta}^{-}$. Si $\sigma_{1}$ et $\sigma_{2}=\mathrm{T}\left(\sigma_{1}\right)$ sont les extrémités de $\alpha$, la brique $\beta^{\prime}$ contient les arêtes $\alpha_{1}^{\prime}$ et $\alpha_{2}^{\prime}=\mathrm{T} \alpha_{1}^{\prime}$ d'image $\widetilde{\alpha}^{\prime}$ par $\pi$ dont $\sigma_{1}$ et $\sigma_{2}$ sont les extrémités. On sait, d'après ce qui précède appliqué à $\alpha_{2}^{\prime}$, que la partie $\bigcup_{k \in \mathbf{Z}} \mathrm{T}^{k} \beta^{\prime}$ n'est pas libre. On est dans le cas où $\beta^{\prime} \in \mathrm{X}_{\beta}^{-}$. Supposons que $f\left(\beta^{\prime}\right) \cap \beta=\emptyset$. Comme précédemment on sait que $\varphi\left(\left\{\beta^{\prime}\right\}\right) \subset \mathrm{X}_{\beta}^{+}$et plus généralement que $\varphi\left(\bigcup_{k \in \mathbf{Z}} \mathrm{T}^{k} \beta^{\prime}\right) \subset \mathrm{X}_{\beta}^{+}$, ce qui est impossible puisque $\bigcup_{k \in \mathbf{Z}} \mathrm{T}^{k} \beta^{\prime}$ est inclus dans $\mathrm{X}_{\beta}^{-}$et n'est pas libre.

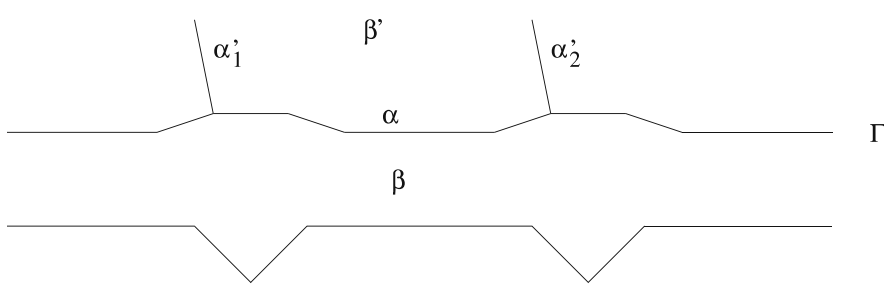


Orientation du squelette

Nous allons maintenant construire une orientation naturelle équivariante $\mathrm{du}$ squelette de $\mathscr{D}$ et donc une orientation de celui de $\widetilde{\mathscr{D}}$.

Considérons une arête $\alpha \in \mathrm{A}$ et supposons que les briques $\beta, \beta^{\prime}$ adjacentes à $\alpha$ aient des images distinctes dans $\tilde{\mathrm{M}}$. Nous savons d'une part que $\beta \cup \beta^{\prime}$ n'est pas libre (par la proposition précédente) et d'autre part que $f\left(\beta^{\prime}\right) \cap \beta=\emptyset$ si $f(\beta) \cap \beta^{\prime} \neq \varnothing$ (par la proposition 2.1). Il existe donc une unique orientation de $\alpha$ telle que $f(g(\alpha)) \cap d(\alpha) \neq \emptyset$, où $g(\alpha)$ (resp. $d(\alpha))$ est la brique adjacente située à gauche (resp. à droite) de $\alpha$.

Supposons maintenant que $\beta^{\prime}=\mathrm{T} \beta$, où $\mathrm{T} \in \mathrm{G}^{*}$. Nous savons que $\bigcup_{k \in \mathbf{Z}} \mathrm{T}^{k} \beta$ n'est pas libre et donc que l'ensemble

$$
\mathrm{Z}=\left\{k \in \mathbf{Z} \mid \mathrm{T}^{k} \beta \in \bigcup_{n \geq 1} \varphi^{n}(\{\beta\})\right\}
$$

n'est pas vide. Cet ensemble est stable par addition et ne contient pas 0 d'après la proposition 2.1. Il est donc formé d'entiers ayant tous le même signe. Si ce signe est positif, nous choisirons l'orientation de $\alpha$ telle que $g(\alpha)=\beta$ et $d(\alpha)=\mathrm{T} \beta$; si ce signe est négatif, nous choisirons l'orientation inverse. Ainsi, si $d(\alpha)=\mathrm{T}(g(\alpha))$, il existe $k \geq 1$ tel que $f(g(\alpha)) \cap \mathrm{T}^{k} d(\alpha) \neq \emptyset$. L'orientation que nous venons de définir sur $\bar{\Sigma}(\mathscr{D})$ est clairement équivariante et définit une orientation sur $\Sigma(\widetilde{\mathscr{D}})$.

L'orientation de $\alpha \in \mathrm{A}$ (ou de $\alpha \in \widetilde{\mathrm{A}}$ ) permet de distinguer, parmi les extrémités de $\alpha$, la source $s(\alpha)$ et le but $\underset{\sim}{b}(\alpha)$, qui sont d'ailleurs distinctes pour $\alpha \in \mathrm{A}$ (mais pas nécessairement pour $\alpha \in \widetilde{\mathrm{A}}$ ).

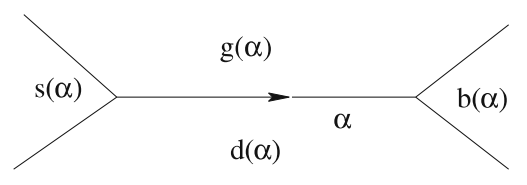

Suites admissibles, $\mathscr{D}$-arcs bien orientés

On dira qu'une suite $\left(\alpha_{i}\right)_{i \in \mathrm{I}}$ d'arêtes de $\mathrm{A}$ (ou de $\widetilde{\mathrm{A}}$ ), indexée par un intervalle I de $\mathbf{Z}$, est admissible si $b\left(\alpha_{i}\right)=s\left(\alpha_{i+1}\right)$ quand $i$ et $i+1$ appartiennent à I. On peut définir naturellement un arc géométrique orienté $\Gamma=\prod_{i \in \mathrm{I}} \alpha_{i}$ par assemblage des arêtes, dont l'orientation coïncide avec celle des arêtes : on dira que $\Gamma$ est bien orienté. Pour un tel arc, on peut définir deux parties de $\mathrm{B}$ (ou de $\widetilde{\mathrm{B}}$ ), le voisinage à droite

$$
d(\Gamma)=\left\{d\left(\alpha_{i}\right), i \in \mathrm{I}\right\}
$$

et le voisinage à gauche

$$
g(\Gamma)=\left\{g\left(\alpha_{i}\right), i \in \mathrm{I}\right\} .
$$


Orientation de la frontière d'une brique

Examinons maintenant l'orientation de la frontière d'une brique. On va étudier successivement la situation dans chacun des trois types de briques. Les propositions 2.8, 2.9 et 2.10 correspondent aux cas i), ii) et iii) de la proposition 2.6. Les résultats qui suivent sont de très légères modifications des résultats de Sauzet sur l'orientation du bord des briques pour une décomposition libre maximale du plan.

Proposition 2.8. - Si $\beta \in \mathrm{B}$ est un disque fermé, sa frontière est réunion de deux segments bien orientés

$$
\Gamma=\prod_{i=0}^{n} \alpha_{i}, \quad \Gamma^{\prime}=\prod_{i=0}^{n^{\prime}} \alpha_{i}^{\prime},
$$

de source commune

$$
s\left(\alpha_{0}\right)=s\left(\alpha_{0}^{\prime}\right)=s(\beta),
$$

appelée source de $\beta$, et de but commun

$$
b\left(\alpha_{n}\right)=b\left(\alpha_{n^{\prime}}^{\prime}\right)=b(\beta),
$$

appelé but de $\beta$, tels que

$$
g(\Gamma)=d\left(\Gamma^{\prime}\right)=\{\beta\} .
$$

En d'autres termes, il existe toujours une arête $\alpha$ telle que $g(\alpha)=\beta$, une arête $\alpha^{\prime}$ telle que $d\left(\alpha^{\prime}\right)=\beta$, et les arêtes du premier type ne sont pas séparées sur $\partial \beta$ par les arêtes $d u$ second type.

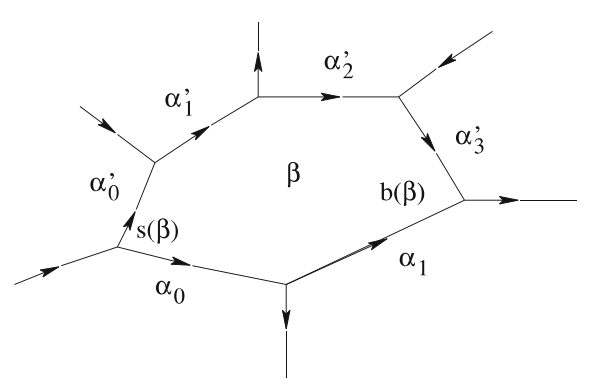

Démonstration. - Supposons d'abord que pour toute arête $\alpha \subset \partial \beta$, on ait $d(\alpha)=\beta$. Fixons $\alpha_{0} \subset \partial \beta$. La brique $\beta_{0}=g(\alpha)$ n'est pas dans la G-orbite de $\beta$, puisque $g\left(\mathrm{~T}^{-1}(\alpha)\right) \neq \beta$ pour tout $\mathrm{T} \in \mathrm{G}$. Ainsi $f\left(\beta_{0}\right)$ rencontre $\beta$ par définition de l'orientation du squelette. On sait d'autre part que $f\left(\alpha_{0}\right) \cap \beta=\emptyset$ puisque $\beta$ est 
libre et $\alpha_{0} \subset \beta$. On en déduit que $f\left(\beta_{0}\right)$, qui est connexe, rencontre une brique $\beta_{1}$ adjacente à $\beta$. En réitérant le raisonnement, on construit alors par récurrence une suite $\left(\beta_{i}\right)_{i \geq 0}$ de briques adjacentes à $\beta$ telle que $f\left(\beta_{i}\right) \cap \beta_{i+1} \neq \emptyset$. Puisqu'il n'y a qu'un nombre fini de telles briques, la proposition 2.1 est contredite. On démontre de même qu'il existe une arête $\alpha^{\prime} \subset \partial \beta$ telle que $d\left(\alpha^{\prime}\right)=\beta$.

Montrons maintenant, toujours par l'absurde, qu'il est impossible de trouver deux arêtes $\alpha_{1}, \alpha_{2}$ vérifiant

$$
g\left(\alpha_{1}\right)=g\left(\alpha_{2}\right)=\beta,
$$

séparées sur $\partial \beta$ par deux arêtes $\alpha_{1}^{\prime}, \alpha_{2}^{\prime}$ vérifiant

$$
d\left(\alpha_{1}^{\prime}\right)=d\left(\alpha_{2}^{\prime}\right)=\beta .
$$

Posons

$$
\begin{cases}\mathrm{X}_{1}=\left\{d\left(\alpha_{1}\right)\right\} & \text { si } d\left(\alpha_{1}\right) \text { n'est pas dans la G-orbite de } \beta \\ \mathrm{X}_{1}=\left\{\mathrm{T}_{1}^{k} \beta, k \geq 1\right\} & \text { si } d\left(\alpha_{1}\right)=\mathrm{T}_{1} \beta\end{cases}
$$

Définissons de façon analogue l'ensemble $\mathrm{X}_{2}$, et aussi les ensembles $\mathrm{X}_{1}^{\prime}$ et $\mathrm{X}_{2}^{\prime}$ en remplaçant la droite par la gauche $(d$ par $g)$. Les parties

$$
\varphi(\{\beta\}) \cup \mathrm{X}_{1} \cup \mathrm{X}_{2}, \quad \varphi_{-}(\{\beta\}) \cup \mathrm{X}_{1}^{\prime} \cup \mathrm{X}_{2}^{\prime}
$$

sont connexes et ne contiennent pas $\beta$. Si nous montrons que ces deux ensembles sont disjoints dans $\mathrm{B}$, nous pourrons construire deux segments $\gamma$ et $\gamma^{\prime}$, le premier joignant un point $z_{1} \in \operatorname{int}\left(\alpha_{1}\right)$ à un point $z_{2} \in \operatorname{int}\left(\alpha_{2}\right)$, à valeurs, sauf aux extrémités, dans l'intérieur de $\varphi(\{\beta\}) \cup \mathrm{X}_{1} \cup \mathrm{X}_{2}$, le second joignant un point $z_{1}^{\prime} \in \operatorname{int}\left(\alpha_{1}^{\prime}\right)$ à un point $z_{2}^{\prime} \in \operatorname{int}\left(\alpha_{2}^{\prime}\right)$, à valeurs, sauf aux extrémités, dans l'intérieur de $\varphi_{-}(\{\beta\}) \cup \mathrm{X}_{1}^{\prime} \cup \mathrm{X}_{2}^{\prime}$. On peut ensuite joindre $z_{1}$ à $z_{2}$ par un segment $\widehat{\gamma}$, à valeurs, sauf aux extrémités, dans $\operatorname{Int}(\beta)$, et de même joindre $z_{1}^{\prime}$ à $z_{2}^{\prime}$ par un segment $\widehat{\gamma}^{\prime}$, à valeurs, sauf aux extrémités, dans $\operatorname{Int}(\beta)$ et on peut supposer que ces deux segments ont un unique point d'intersection avec une intersection transverse. On obtient deux cercles $\gamma \cup \widehat{\gamma}$ et $\gamma^{\prime} \cup \widehat{\gamma}^{\prime}$ ayant un unique point d'intersection avec une intersection transverse. Ceci est impossible.

Il reste donc à montrer (dans $\mathrm{B}$ ) que chacune des relations

$$
\mathrm{X}_{1} \cap \mathrm{X}_{1}^{\prime} \neq \emptyset, \quad \varphi(\{\beta\}) \cap \mathrm{X}_{1}^{\prime} \neq \varnothing
$$

aboutit à une contradiction.

La seconde est impossible si $\mathrm{X}_{1}^{\prime}=\left\{g\left(\alpha_{1}^{\prime}\right)\right\}$ d'après la proposition 2.1. En effet on sait, par définition de l'orientation du squelette, que $\beta \in \varphi\left(\left\{g\left(\alpha_{1}^{\prime}\right)\right\}\right.$. Elle 
est également impossible si $\mathrm{X}_{1}^{\prime}=\left\{\mathrm{T}_{1}^{\prime k} \beta, k \geq 1\right\}$. En effet, on a vu quand on a orienté le squelette, et toujours grâce à la proposition 2.1, que l'ensemble

$$
\mathrm{Z}=\left\{k \in \mathbf{Z} \mid \mathrm{T}_{1}^{\prime k} \beta \in \bigcup_{n \geq 1} \varphi^{n}(\{\beta\})\right\}
$$

ne contient aucun nombre positif.

La relation $\mathrm{X}_{1} \cap \mathrm{X}_{1}^{\prime} \neq \emptyset$ est évidemment impossible si l'une exactement des deux briques $d\left(\alpha_{1}\right), g\left(\alpha_{1}^{\prime}\right)$ est dans la G-orbite de $\beta$. Elle est impossible, d'après la proposition 2.1 et la définition de l'orientation en $\alpha_{1}$ et $\alpha_{1}^{\prime}$ si aucune des deux briques n'est dans la G-orbite de $\beta$. Supposons donc que

$$
d\left(\alpha_{1}\right)=\mathrm{T}_{1} \beta, \quad g\left(\alpha_{1}^{\prime}\right)=\mathrm{T}_{1}^{\prime} \beta .
$$

Si $\mathrm{X}_{1} \cap \mathrm{X}_{1}^{\prime} \neq \emptyset$, alors il existe $n>0$ et $n^{\prime}>0$ tel que $\mathrm{T}_{1}^{n} \beta=\mathrm{T}_{1}^{\prime n^{\prime}} \beta$. On en déduit que $\mathrm{T}_{1}^{n}=\mathrm{T}_{1}^{\prime n^{\prime}}$ puisque le stabilisateur de $\beta$ est trivial (car $\beta$ est un disque, voir la proposition 2.6). On sait d'autre part qu'il existe des entiers $m>0$ et $m^{\prime}>0$ tels que

$$
\mathrm{T}_{1}^{m} \beta \in \varphi(\{\beta\}), \quad \mathrm{T}_{1}^{\prime m^{\prime}} \beta \in \varphi_{-}(\{\beta\})
$$

On en déduit :

$$
\mathrm{T}_{1}^{m m^{\prime} n} \beta=\mathrm{T}_{1}^{\prime m m^{\prime} n^{\prime}} \beta \in\left(\bigcup_{k \geq 1} \varphi^{k}(\{\beta\})\right) \bigcap\left(\bigcup_{k \geq 1} \varphi_{-}^{k}(\{\beta\})\right),
$$

ce qui contredit, là encore, la proposition 2.1.

Proposition 2.9. - Soit $\beta \in \mathrm{B}$ un demi-plan fermé. Deux cas sont alors possibles :

i) La frontière de $\beta$ est la réunion de deux demi-droites positives bien orientées

$$
\Gamma=\prod_{i=0}^{n} \alpha_{i}, \quad \Gamma^{\prime}=\prod_{i=0}^{n^{\prime}} \alpha_{i}^{\prime}
$$

où $n \leq+\infty$ et $n^{\prime} \leq+\infty$, de source commune

$$
s\left(\alpha_{0}\right)=s\left(\alpha_{0}^{\prime}\right)=s(\beta),
$$

appelée source de $\beta$, telles que

$$
g(\Gamma)=d\left(\Gamma^{\prime}\right)=\{\beta\} .
$$


ii) La frontière de $\beta$ est la réunion de deux demi-droites négatives bien orientées

$$
\Gamma=\prod_{i=n}^{0} \alpha_{i}, \quad \Gamma^{\prime}=\prod_{i=n^{\prime}}^{0} \alpha_{i}^{\prime},
$$

où $n \geq-\infty$ et $n^{\prime} \geq-\infty$, de but commun

$$
b\left(\alpha_{0}\right)=b\left(\alpha_{0}^{\prime}\right)=b(\beta),
$$

appelé but de $\beta$, telles que

$$
g(\Gamma)=d\left(\Gamma^{\prime}\right)=\{\beta\} .
$$
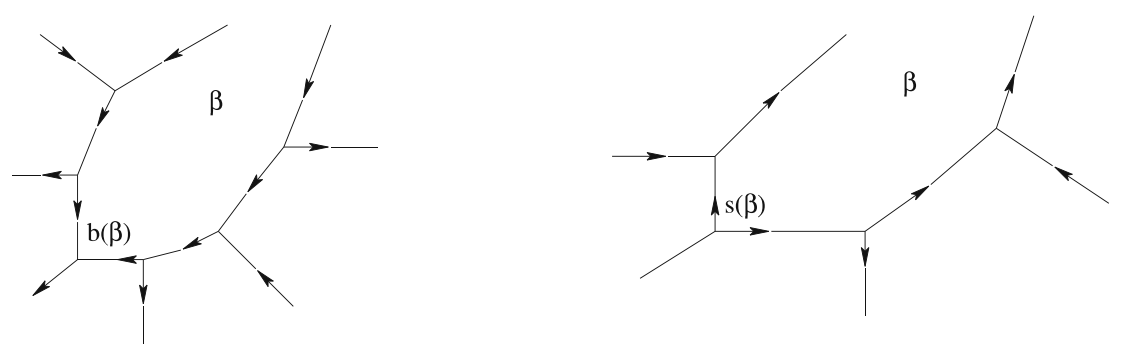

Démonstration. - Commençons par démontrer qu'il existe au moins une arête $\alpha$ telle que $g(\alpha)=b$ et une arête $\alpha^{\prime}$ telle que $d\left(\alpha^{\prime}\right)=b$. On raisonne par l'absurde et on suppose que toute arête $\alpha \subset \partial \beta$ vérifie $d(\alpha)=\beta$. Le raisonnement qui suit est dû à Sauzet [Sa].

La frontière de $\beta$ s'écrit $\Gamma=\prod_{k<i<m} \alpha_{i}$, où $-\infty \leq l<m \leq+\infty$, et où la suite $\left(a_{i}\right)_{l<i<m}$ est admissible. Pour les raisons évoquées au tout début de la démonstration de la proposition 2.8, on sait que $g\left(\alpha_{i}\right)$ et $d\left(\alpha_{i}\right)=\beta$ ne sont pas dans la même G-orbite (et donc que $f\left(g\left(\alpha_{i}\right)\right) \cap \beta \neq \emptyset$ ) et également qu'il y a un nombre infini d'arêtes $\alpha_{i}$. Remarquons que $\beta=d(\Gamma)=\mathrm{D}(\Gamma)$ et que les ensembles $\beta, f^{-1}(\beta)$ et $f(\beta)$ sont disjoints deux à deux. On pose alors $\beta_{i}=g\left(\alpha_{i}\right)$ et $\sigma_{i}=s\left(\alpha_{i}\right)$. Fixons $i_{0}$ entre $l$ et $m$. On peut trouver un segment $\gamma$ joignant $\sigma_{i_{0}}$ à $f\left(\sigma_{i_{0}}\right)$ et qui est disjoint, sauf aux extrémités, de $\beta \cup f(\beta) \cup f^{-1}(\beta)$. L'ensemble $f^{-1}(\beta)$ est alors séparé dans $\mathrm{G}(\Gamma) \cap f(\mathrm{G}(\Gamma))$ par $\gamma$, de toutes les arêtes $\alpha_{i}, i_{0} \leq i<m$, ou de toutes les arêtes $\alpha_{i}, l<i<i_{0}$. Plaçons nous dans le premier cas, le second étant analogue. Toute brique $\beta_{i}, i_{0} \leq i<m$, rencontre $f^{-1}(\beta)$. La propriété 2.1 nous dit qu'elle ne rencontre pas $f(\beta)$ et est donc contenue dans $\mathrm{G}(\Gamma) \cap f(\mathrm{G}(\Gamma))$. Ainsi, toute brique $\beta_{i}, i_{0} \leq i<m$, rencontre $\gamma$. Il n'y a donc qu'un nombre fini de telles briques. Un argument similaire à celui donné dans la démonstration de la proposition précédente et aboutissant à la construction de deux cercles s'inter- 
sectant en un unique point, et ceci de façon transverse, nous donne le résultat suivant :

$$
\text { si } \beta_{i_{1}}=\beta_{i_{2}} \neq \beta_{i_{3}}=\beta_{i_{4}} \text {, où } l<i_{1}<i_{3}<i_{2}<m \text {, alors } i_{1}<i_{4}<i_{2} \text {. }
$$

On en déduit que les arêtes $\alpha_{i}, i_{0} \leq i<m$ sont en nombre fini.

Pour obtenir une contradiction, il suffit de trouver deux parties connexes disjointes de $\mathrm{B}$, la première contenant $\beta$ et $f(\beta)$, la seconde contenant $\beta_{m-1}$ et $f^{-1}\left(\beta_{m-1}\right)$. Soit $\alpha$ l'arête distincte de $\alpha_{m-2}$ et de $\alpha_{m-1}$ dont $\sigma_{m-1}$ est un sommet. Supposons d'abord que $\sigma_{m-1}=s(\alpha)$ et que $\beta_{m-2}$ n'est pas dans la G-orbite de $\beta_{m-1}$. On en déduit que $\varphi_{-}\left(\left\{\beta_{m-1}\right\}\right)$ contient $\beta_{m-2}$ puis que $\beta_{m-1} \cup \varphi_{-}\left(\left\{\beta_{m-1}\right\}\right)$ est connexe (puisque la brique $\beta_{m-2}$ est adjacente à $\beta_{m-1}$ ). L'ensemble

$$
\mathrm{X}=\bigcup_{n \geq 0} \varphi_{-}^{n}\left(\left\{\beta_{m-1}\right\}\right)
$$

est un répulseur, qui contient $\beta_{m-1}$ et $f^{-1}\left(\beta_{m-1}\right)$. Il est connexe, car on peut écrire

$$
\mathrm{X}=\bigcup_{n \geq 0} \varphi_{-}^{n}\left(\left\{\beta_{m-1}\right\} \cup \varphi_{-}\left(\left\{\beta_{m-1}\right\}\right)\right)
$$

comme réunion de parties connexes $\mathrm{X}_{n}=\varphi_{-}^{n}\left(\left\{\beta_{m-1}\right\} \cup \varphi_{-}\left(\left\{\beta_{m-1}\right\}\right)\right)$ telles que $\mathrm{X}_{n} \cap \mathrm{X}_{n+1} \neq \emptyset$. De même, l'ensemble

$$
\mathrm{X}^{\prime}=\bigcup_{n \geq 1} \varphi^{n}\left(\left\{\beta_{m-1}\right\}\right)=\bigcup_{n \geq 1} \varphi^{n}\left(\left\{\beta_{m-1}\right\} \cup \varphi\left(\left\{\beta_{m-1}\right\}\right)\right)
$$

est un attracteur connexe qui contient $\beta$ puisqu'on sait que $f\left(\beta_{m-1}\right)$ rencontre $\beta$. L'ensemble $\mathrm{X}^{\prime}$ contient donc également $f(\beta)$ et est disjoint dans $\mathrm{B}$ de $\mathrm{X}$.

Supposons maintenant que $\sigma_{m-1}=s(\alpha)$ et que $\beta_{m-2}=\mathrm{T} \beta_{m-1}, \mathrm{~T} \in \mathrm{G}^{*}$. On vérifie que

$$
\mathrm{X}=\bigcup_{n, k \geq 0} \varphi_{-}^{n}\left(\mathrm{~T}^{k}\{\beta\}\right)
$$

est un répulseur connexe, qui contient $\beta_{m-1}$ et $f^{-1}\left(\beta_{m-1}\right)$ et qui est disjoint de l'attracteur $\mathrm{X}^{\prime}$ défini plus haut.

$\mathrm{Si}$, par contre, $s\left(\alpha_{m-1}\right)=b(\alpha)$, on définit les ensembles $\mathrm{X}$ et $\mathrm{X}^{\prime}$ à partir de $\beta_{m-2}$ et pas de $\beta_{m-1}$ en intervertissant le rôle des deux briques, le répulseur $\mathrm{X}$ contiendra $\beta_{m-1}$ et $f^{-1}\left(\beta_{m-1}\right)$. 

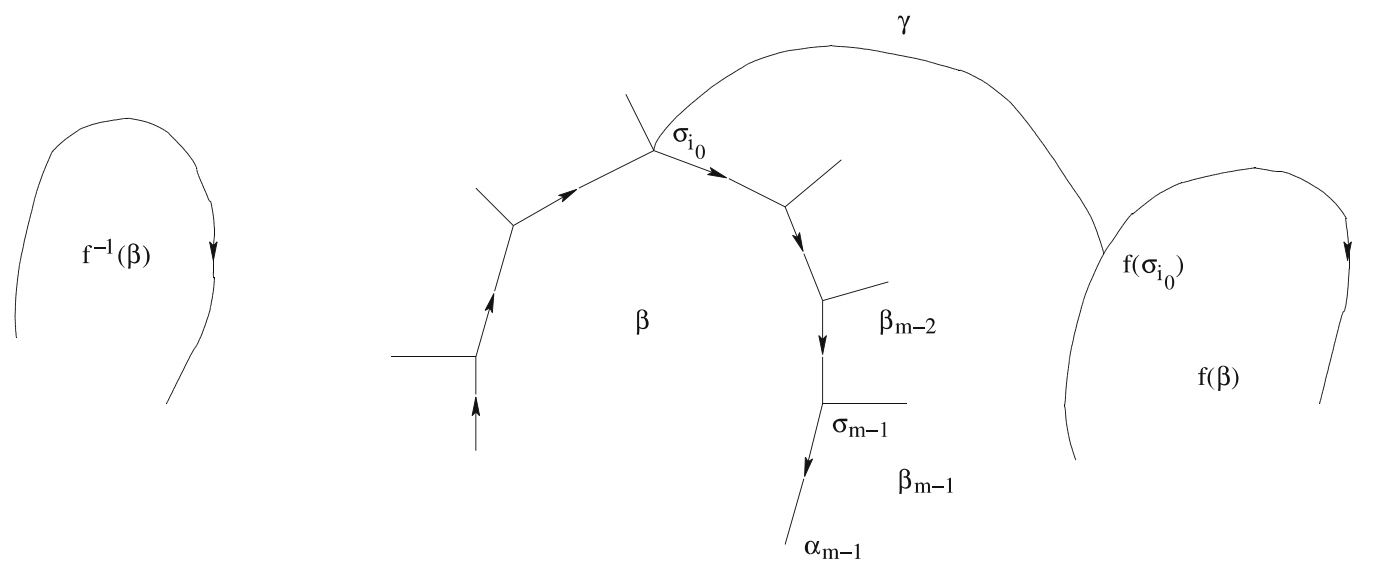

Il y a donc deux types d'arêtes sur la frontière de $\beta$, les arêtes $\alpha$ telles que $g(\alpha)=\beta$ et les arêtes $\alpha^{\prime}$ telles que $d\left(\alpha^{\prime}\right)=\beta$. Pour conclure il reste à montrer que deux arêtes d'un certain type ne peuvent pas être séparées sur la frontière de $\beta$ par une arête de l'autre type. Supposons, par exemple, que l'on puisse trouver deux arêtes $\alpha_{1}, \alpha_{2}$ telles que $g\left(\alpha_{1}\right)=g\left(\alpha_{2}\right)=\beta$, séparées sur $\partial \beta$ par une arête $\alpha_{1}^{\prime}$ vérifiant $d\left(\alpha_{1}^{\prime}\right)=\beta$. Comme dans la preuve de la proposition 2.8, on pose

$$
\begin{cases}\mathrm{X}_{1}=\left\{d\left(\alpha_{1}\right)\right\} & \text { si } d\left(\alpha_{1}\right) \text { n'est pas dans la G-orbite de } \beta, \\ \mathrm{X}_{1}=\left\{\mathrm{T}_{1}^{k} \beta, k \geq 1\right\} & \text { si } d\left(\alpha_{1}\right)=\mathrm{T}_{1} \beta .\end{cases}
$$

On définit de façon analogue l'ensemble $\mathrm{X}_{2}$, et aussi l'ensemble $\mathrm{X}_{1}^{\prime}$ en remplaçant la droite par la gauche $(d$ par $g)$. Par le même raisonnement que dans la preuve de la proposition 2.8, en utilisant en particulier le fait que le stabilisateur de $\beta$ est trivial (voir proposition 2.6), on prouve que les parties

$$
\varphi(\{\beta\}) \cup \mathrm{X}_{1} \cup \mathrm{X}_{2}, \quad \varphi_{-}(\{\beta\}) \cup \mathrm{X}_{1}^{\prime},
$$

qui sont connexes et ne contiennent pas $\beta$, sont en fait disjointes dans B. Considérons un segment $\gamma$ (resp. $\widehat{\gamma}$ ) joignant un point $z_{1} \in \operatorname{int}\left(\alpha_{1}\right)$ à un point $z_{2} \in \operatorname{int}\left(\alpha_{2}\right)$, à valeurs, sauf aux extrémités, dans l'intérieur de $\varphi(\{\beta\}) \cup \mathrm{X}_{1} \cup \mathrm{X}_{2}$ (resp. dans l'intérieur de $\beta)$. L'ensemble $\varphi_{-}(\{\beta\}) \cup \mathrm{X}_{1}^{\prime}$ est alors inclus dans la composante connexe bornée du complémentaire du cercle $\gamma \widehat{\gamma}$. Or $\varphi_{-}(\{\beta\}) \cup \mathrm{X}_{1}^{\prime}$ n'est pas borné puisqu'il contient $f^{-1}(\beta)$. Nous avons donc une contradiction.

Proposition 2.10. - Soit $\beta \in \mathrm{B}$ une bande fermée. La droite $\partial \mathrm{X}_{\beta}^{+}$s'écrit alors $\partial \mathrm{X}_{\beta}^{+}=\prod_{i \in \mathrm{I}} \alpha_{i}$, où $\mathrm{I}$ est un intervalle de $\mathbf{Z}$. Elle est bien orientée et on a $g\left(\partial \mathrm{X}_{\beta}^{+}\right)=\{\beta\}$. La droite $\partial \mathrm{X}_{\beta}^{-}$s'écrit alors $\partial \mathrm{X}_{\beta}^{-}=\prod_{i \in I^{\prime}} \alpha_{i}^{\prime}$, où $\mathrm{I}^{\prime}$ est un intervalle de $\mathbf{Z}$. Elle est bien orientée et on $\operatorname{ad}\left(\partial \mathrm{X}_{\beta}^{-}\right)=\{\beta\}$. 


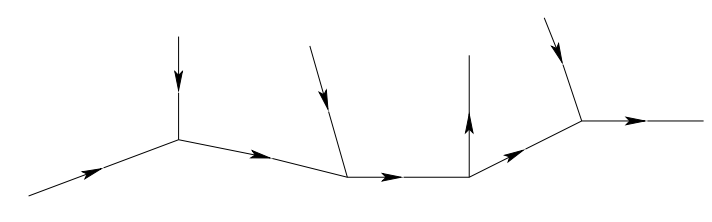

$\beta$

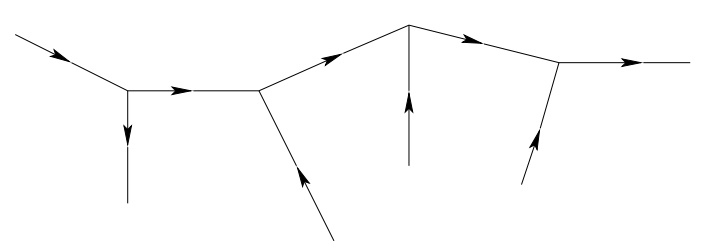

Démonstration. - Soit $\alpha$ une arête telle que $g(\alpha)=\beta$. Si $d(\alpha)$ n'est pas dans la G-orbite de $\beta$, alors $f(\beta) \cap d(\alpha) \neq \emptyset$ par définition de l'orientation du squelette. La brique $d(\alpha)$ se trouve donc dans la composante connexe de $\mathrm{B} \backslash\{\beta\}$ qui contient $\varphi(\{\beta\})$, ̀̀ savoir dans $\mathrm{X}_{\beta}^{+}$. Ainsi $\alpha$ est contenue dans $\partial \mathrm{X}_{\beta}^{+}$. Si $d(\alpha)=\mathrm{T}(\beta), \mathrm{T} \in \mathrm{G}^{*}$, alors $f(\beta)$ rencontre $\bigcup_{k \geq 1} \mathrm{~T}^{k} \beta$. Ainsi, l'ensemble connexe $\bigcup_{k \geq 1} \mathrm{~T}^{k}(\beta)$ est inclus dans $\mathrm{X}_{\beta}^{+}$ et l'arête $\alpha$ contenue dans $\partial \mathrm{X}_{\beta}^{+}$. On démontre de même que si $d(\alpha)=\beta$, alors $\alpha \subset \partial \mathrm{X}_{\beta}^{-}$.

Orientation du squelette au voisinage d'un sommet, existence de $\mathscr{D}$-droites bien orientées

Proposition 2.11. - Tout sommet $\sigma \in \mathrm{S}$ est source d'une arête et but de deux arêtes ou alors but d'une arête et source de deux arêtes. Dans le premier cas, $\sigma$ est le but d'une brique, dans le second cas, c'est la source d'une brique.

Démonstration. - Supposons que $\sigma$ soit le but de trois arêtes $\alpha_{1}, \alpha_{2}, \alpha_{3}$, indexées de façon telle que

$$
d\left(\alpha_{1}\right)=g\left(\alpha_{2}\right)=\beta_{1}, \quad d\left(\alpha_{2}\right)=g\left(\alpha_{3}\right)=\beta_{2}, \quad d\left(\alpha_{3}\right)=g\left(\alpha_{1}\right)=\beta_{3} .
$$

Puisqu'une brique $\beta$ a au plus un but (voir propositions 2.8, 2.9 et 2.10), les trois briques $\pi\left(\beta_{1}\right), \pi\left(\beta_{2}\right)$ et $\pi\left(\beta_{3}\right)$ sont distinctes. En effet, si $\beta_{2}=\mathrm{T} \beta_{1}$, alors $\sigma$ et $\mathrm{T}^{-1} \sigma$ sont deux buts distincts de $\beta_{1}$. On en déduit (via la proposition 2.7) que

$$
\beta_{2} \in \varphi\left(\left\{\beta_{1}\right\}\right), \quad \beta_{3} \in \varphi\left(\left\{\beta_{2}\right\}\right), \quad \beta_{1} \in \varphi\left(\left\{\beta_{3}\right\}\right),
$$

ce qui contredit la proposition 2.1. On démontre de même que $\sigma$ n'est pas la source de trois arêtes.

La proposition nous dit en particulier que toute arête $\alpha \in \mathrm{A}$ peut être prolongée en un arc bien orienté. Plus précisément, il existe une suite admissible $\left(\alpha_{i}\right)_{n<i<m}$, où $\alpha_{0}=\alpha$, où toutes les arêtes $\alpha_{i}$ sont des segments, sauf $\alpha_{n+1}$, dans 
le cas où $n>-\infty$, qui est une demi-droite négative, et $\alpha_{m-1}$, dans le cas où $m<+\infty$, qui est une demi-droite positive. Nous allons voir que l'arc $\prod_{n<i<m} \alpha_{i}$ est nécessairement simple, c'est une $\mathscr{D}$-droite.

Proposition 2.12. - Il n'existe pas de $\mathscr{D}$-cercle bien orienté.

Démonstration. - Supposons que $\Gamma$ soit un $\mathscr{D}$-cercle bien orienté. Ce cercle est la frontière d'un disque $\mathrm{X} \subset \mathrm{B}$. Nous supposerons que $\mathrm{X}=\mathrm{D}(\Gamma)$, nous savons donc que $d(\Gamma) \subset \mathrm{X}$ et $g(\Gamma) \subset \mathrm{B} \backslash \mathrm{X}$. Pour toute arête $\alpha \subset \Gamma$, les briques $d(\alpha)$ et $g(\alpha)$ ne sont pas dans la même G-orbite. En effet, si $d(\alpha)=\mathrm{T} g(\alpha)$, une récurrence immédiate sur $n \geq 1$, donne l'inclusion

$$
\bigcup_{1 \leq k \leq n} \mathrm{~T}^{k}(g(\alpha)) \subset \mathrm{X}
$$

qui contredit le caractère fini de X. On a donc $d(\alpha) \subset \varphi(\{g(\alpha)\})$. Ainsi, pour toute brique $\beta \in g(\Gamma)$, l'ensemble $\bigcup_{n \geq 1} \varphi^{n}(\{\beta\})$ rencontre $\mathrm{X}$ et est infini puisque c'est un attracteur. Il est connexe, puisque $\{\beta\} \cup \varphi(\{\beta\})$ est connexe et qu'on peut écrire

$$
\bigcup_{n \geq 1} \varphi^{n}(\{\beta\})=\bigcup_{n \geq 1} \varphi^{n}(\{\beta\} \cup \varphi(\{\beta\})),
$$

comme réunion de parties connexes $\mathrm{X}_{n}=\varphi^{n}(\{\beta\} \cup \varphi(\{\beta\}))$ telles que $\mathrm{X}_{n} \cap \mathrm{X}_{n+1} \neq \emptyset$. Cet ensemble contient donc une brique $\beta^{\prime} \in g(\Gamma)$. On construit alors une chaîne fermée de briques dans $g(\Gamma)$, comme dans la démonstration de la proposition 2.8.

Seconde action de l'homéomorphisme sur la décomposition

On va compléter maintenant les travaux de Sauzet. On définit une application

$$
\psi: \mathscr{P}(\mathrm{B}) \rightarrow \mathscr{P}(\mathrm{B})
$$

en posant :

$$
\psi(\mathrm{X})=\{\beta \in \mathrm{B} \mid \text { il existe } \alpha \in \mathrm{A} \text { telle que } g(\alpha) \in \mathrm{X} \text { et } d(\alpha)=\beta\} .
$$

Remarquons, là encore, que $\psi(\mathrm{X})$ est connexe si c'est le cas de $\mathrm{X}$, et que pour toute famille $\left(\mathrm{X}_{i}\right)_{i \in \mathrm{I}}$ de parties de $\mathrm{B}$, on a :

$$
\psi\left(\bigcup_{i \in \mathrm{I}} \mathrm{X}_{i}\right)=\bigcup_{i \in \mathrm{I}} \psi\left(\mathrm{X}_{i}\right), \quad \psi\left(\bigcap_{i \in \mathrm{I}} \mathrm{X}_{i}\right) \subset \bigcap_{i \in \mathrm{I}} \psi\left(\mathrm{X}_{i}\right) .
$$


On appellera partie droite toute partie $\mathrm{X} \subset \mathrm{B}$ vérifiant $\psi(\mathrm{X}) \subset \mathrm{X}$. En d'autres termes, ceci signifie que pour toute arête $\alpha \subset \partial \mathrm{X}$, on a $d(\alpha) \in \mathrm{X}$ et $g(\alpha) \in \mathrm{B} \backslash \mathrm{X}$. Les composantes connexes de $\partial \mathrm{X}$ sont des $\mathscr{D}$-droites bien orientées (d'après la proposition 2.12). La réunion et l'intersection d'une famille de parties droites est encore une partie droite.

On définit de façon analogue une application

$$
\psi_{-}: \mathscr{P}(\mathrm{B}) \rightarrow \mathscr{P}(\mathrm{B})
$$

en posant :

$$
\psi_{-}(\mathrm{X})=\{\beta \in \mathrm{B} \mid \text { il existe } \alpha \in \mathrm{A} \text { telle que } d(\alpha) \in \mathrm{X} \text { et } g(\alpha)=\beta\}
$$

Là encore, les relations

$$
\beta^{\prime} \in \psi^{n}(\{\beta\}), \quad \beta \in \psi_{-}^{n}\left(\left\{\beta^{\prime}\right\}\right)
$$

sont équivalentes. Une partie gauche est une partie positivement invariante par $\psi_{-}$, c'est le complémentaire d'une partie droite.

Bons attracteurs, bons répulseurs

Un bon attracteur est par définition une partie $\mathrm{X} \subset \mathrm{B}$ positivement invariante à la fois par $\varphi$ et par $\psi$, c'est-à-dire invariante par $\varphi \cup \psi$. De même un bon répulseur est le complémentaire d'un bon attracteur, c'est-à-dire une partie invariante par $\varphi_{-} \cup \psi_{-}$. Une réunion ou une intersection de bons attracteurs (resp. de bons répulseurs) est encore un bon attracteur (resp. un bon répulseur).

Nous allons voir que la proposition 2.1, fondamentale dans notre étude, a un équivalent pour $\psi$ et même pour $\varphi \cup \psi$ :

Proposition 2.13. - Pour toute brique $\beta \in \mathrm{B}$, le bon attracteur $\bigcup_{n \geq 1}(\varphi \cup \psi)^{n}(\{\beta\})$ ne
pas $\beta$. contient pas $\beta$.

Démonstration. - Il faut montrer qu'il n'existe pas de chaîne fermée généralisée, c'est-à-dire de suite finie $\left(\beta_{i}\right)_{i \in \mathbf{Z} / n \mathbf{Z}}$ de briques telles que $\beta_{i+1} \in(\varphi \cup \psi)\left(\left\{\beta_{i}\right\}\right)$. S'il existe une chaîne fermée généralisée, il en existe une $\left(\beta_{i}\right)_{i \in \mathbf{Z} / n \mathbf{Z}}$ dont la "longueur" $n$ est minimale. Cette longueur est au moins égale à 2 et les $\beta_{i}$ sont tous distincts. Nous savons, par la proposition 2.1, qu'il existe $i \in \mathbf{Z} / n \mathbf{Z}$ telle que $\beta_{i+1} \notin \varphi\left(\left\{\beta_{i}\right\}\right)$. Montrons de même qu'il existe $i \in \mathbf{Z} / n \mathbf{Z}$ tel que $\beta_{i+1} \notin \psi\left(\left\{\beta_{i}\right\}\right)$. Dans le cas contraire, on peut trouver $\alpha_{i} \in \mathrm{A}$ tel que $g\left(\alpha_{i}\right)=\beta_{i}, d\left(\alpha_{i}\right)=\beta_{i+1}$, choisir ensuite un point $z_{i} \in \operatorname{int}\left(\alpha_{i}\right)$, enfin construire un segment $\gamma_{i}$ joignant $z_{i}$ à $z_{i+1}$ et inclus, sauf aux extrémités, dans $\operatorname{Int}\left(\beta_{i}\right)$. L'arc $\prod_{i \in \mathbf{Z} / n \mathbf{Z}} \gamma_{i}=\Gamma$ est un cercle transverse au sque- 
lette $\Sigma(\mathscr{D})$. Nous pouvons construire une $\mathscr{D}$-droite bien orientée $\Gamma^{\prime}$ contenant $\alpha_{0}$. Cette droite contient l'arête $\alpha_{0}$ mais ne contient aucune autre arête $\alpha_{i}$. Elle ne rencontre $\Gamma$ qu'au point $z_{0}$ et intersecte transversalement $\Gamma$. Ceci est impossible.

On peut donc trouver un entier $m \in\{1, \ldots, n\}$ et une partition $\left(\mathrm{I}_{l}\right)_{l \in \mathbf{Z} / m \mathbf{Z}}$ de $\mathbf{Z} / n \mathbf{Z}$ en intervalles consécutifs $\mathrm{I}_{l}=\left\{i_{l}, \ldots, i_{l+1}-1\right\}$ tels que :

$-\beta_{i_{l+1}} \in \varphi\left(\left\{\beta_{i_{l+1}-1}\right\}\right)$,

$-\beta_{i} \notin \varphi\left(\left\{\beta_{i-1}\right\}\right)$ si $i_{l}<i<i_{l+1}$.

Supposons $m \geq 2$. Les parties $\mathrm{X}_{l}=\left\{\beta_{i}, i \in \mathrm{I}_{l}\right\}$ sont connexes, puisque $\beta_{i-1}$ et $\beta_{i}$ sont adjacentes, si $i_{l}<i<i_{l+1}$. Elles sont également libres puisque la chaîne est de longueur minimale. Puisque $f\left(\mathrm{X}_{l}\right) \cap \mathrm{X}_{l+1} \neq \emptyset$, ceci contredit le corollaire 2.2.

Supposons $m=1$ et, pour simplifier l'écriture, écrivons $i_{1}=0$. Puisque $\beta_{1} \notin$ $\varphi\left(\left\{\beta_{0}\right\}\right)$, on sait grâce à la proposition 2.7 , qu'il existe $\mathrm{T} \in \mathrm{G}^{*}$ tel que $\beta_{1}=\mathrm{T} \beta_{0}$ et $r \geq 1$ tel que

$$
\begin{aligned}
& -\bigcup_{0 \leq l \leq r} \mathrm{~T}^{-l} \beta_{0} \text { est libre, } \\
& -f\left(\mathrm{~T}^{-r} \beta_{0}\right) \cap \beta_{1} \neq \emptyset .
\end{aligned}
$$

Les ensembles

$$
\mathrm{X}_{0}=\left\{\mathrm{T}^{-l} \beta_{0}, 0 \leq l \leq r\right\}, \quad \mathrm{X}_{1}=\left\{\beta_{i}, 1 \leq i<n\right\}
$$

sont connexes et libres (le second par minimalité de la longueur) et on a

$$
f\left(\mathrm{X}_{0}\right) \cap \mathrm{X}_{1} \neq \emptyset, \quad f\left(\mathrm{X}_{1}\right) \cap \mathrm{X}_{0} \neq \emptyset
$$

Puisqu'il n'existe aucune chaîne fermée généralisée $\left(\beta_{i}^{\prime}\right)_{i \in \mathbf{Z} / n^{\prime} \mathbf{Z}}$ où $\beta_{i+1}^{\prime} \in \psi\left(\left\{\beta_{i}\right\}\right)$, on sait qu'ils sont également disjoints. Ceci contredit le corollaire 2.2.

\section{Recouvrement du squelette par des droites de Brouwer}

Nous allons voir comment recouvrir le squelette de $\mathscr{D}$ par des droites de Brouwer, de façon analogue à ce qui est fait par Sauzet dans sa thèse et repris dans [LeC5]. Remarquons d'abord que toute composante connexe $\mathrm{X}_{1}$ d'un bon attracteur $\mathrm{X}$ est également un bon attracteur. C'est une conséquence essentielle de la minimalité de la décomposition. Pour montrer cela, il suffit de trouver une partie connexe $\mathrm{X}^{\prime} \subset \mathrm{X}$ rencontrant $\mathrm{X}_{1}, \varphi\left(\mathrm{X}_{1}\right)$ et $\psi\left(\mathrm{X}_{1}\right)$. La composante connexe de $\mathrm{X}$ contenant $\mathrm{X}^{\prime}$ sera nécessairement $\mathrm{X}_{1}$ et elle contiendra $\varphi\left(\mathrm{X}_{1}\right) \cup \psi\left(\mathrm{X}_{1}\right)$. On choisit une brique $\beta \in \mathrm{X}_{1}$ et une arête $\alpha \subset \partial \beta$ telle que $g(\alpha)=\beta$. On pose alors $\beta^{\prime}=d(\alpha)$ et on utilise la proposition 2.7 et la définition de l'orientation du squelette. Si $\beta^{\prime}$ n'est pas dans la G-orbite de $\beta$, alors $\beta^{\prime} \in \varphi(\{\beta\}) \cap \psi(\{\beta\})$ et la partie $\mathrm{X}^{\prime}=\left\{\beta, \beta^{\prime}\right\}$ convient ; si $\beta^{\prime}=\mathrm{T} \beta, \mathrm{T} \in \mathrm{G}^{*}$, la partie $\mathrm{X}^{\prime}=\bigcup_{k \geq 0} \mathrm{~T}^{k} \beta$ convient. 
Soit $\mathrm{X}$ un bon attracteur. La frontière de $\mathrm{X}$ est formée de $\mathscr{D}$-droites bien orientées. Soit $\Gamma$ une telle droite. Le voisinage à droite $d(\Gamma)$ est inclus dans une composante connexe $\mathrm{X}_{1}$ de $\mathrm{X}$, qui elle-même est un bon attracteur. L'ensemble $\mathrm{G}(\Gamma)$ est alors une composante connexe du bon répulseur $\mathrm{B} \backslash \mathrm{X}_{1}$, c'est également un bon répulseur et son complémentaire $\mathrm{D}(\Gamma)$ un bon attracteur. Ainsi $\Gamma$ est une $\mathscr{D}$-droite de Brouwer bien orientée. Réciproquement, si $\Gamma$ est une $\mathscr{D}$-droite de Brouwer bien orientée, alors l'ensemble $\mathrm{D}(\Gamma)$ est un bon attracteur. Par définition, on dira que $\Gamma$ est une bonne $\mathscr{D}$-droite de Brouwer.

Toute arête $\alpha \in \mathrm{A}$ est incluse dans une bonne $\mathscr{D}$-droite de Brouwer. En effet, la partie

$$
\mathrm{X}=\bigcup_{n \geq 0}(\varphi \cup \psi)^{n}(\{d(\alpha)\})
$$

est un bon attracteur qui contient $d(\alpha)$ mais ne contient pas $g(\alpha)$ car $d(\alpha) \in$ $\psi(g(\{\alpha\}))$. L'arête $\alpha$ est donc sur la frontière de $\mathrm{X}$ et la droite frontière qui contient $\alpha$ est une bonne $\mathscr{D}$-droite de Brouwer. On peut donc recouvrir le squelette par des bonnes $\mathscr{D}$-droites de Brouwer.

\section{3. Étude du cas abélien et énoncé de la proposition fondamentale}

On dira que deux $\mathscr{D}$-arcs bien orientés $\Gamma=\prod_{i \in \mathrm{I}} \alpha_{i}$ et $\Gamma=\prod_{i \in \mathrm{I}^{\prime}} \alpha_{i}^{\prime}$ ont une intersection transverse s'il existe trois entiers $k, l$, et $m$ avec $l-k \geq 2$ tels que :

$-\{k, \ldots, l\} \subset \mathrm{I}$ et $\{k+m, \ldots, l+m\} \subset \mathrm{I}^{\prime}$;

- pour tout $i \in\{k+1, \ldots, l-1\}, \alpha_{i}=\alpha_{i+m}^{\prime}$;

$-\alpha_{k} \neq \alpha_{k+m}^{\prime}$ et $\alpha_{l} \neq \alpha_{l+m}^{\prime}$;

$-d\left(\alpha_{k}\right)=g\left(\alpha_{k+m}^{\prime}\right) \Longrightarrow g\left(\alpha_{l}\right)=d\left(\alpha_{l+m}^{\prime}\right)$;

$-g\left(\alpha_{k}\right)=d\left(\alpha_{k+m}^{\prime}\right) \Longrightarrow d\left(\alpha_{l}\right)=g\left(\alpha_{l+m}^{\prime}\right)$.

Remarquons que dans le cas de deux $\mathscr{D}$-droites, la définition équivaut à celle donnée au début de l'article (dans la section 0 ). Remarquons également que si $\mathrm{X}$ et $\mathrm{X}^{\prime}$ sont deux bons attracteurs comparables $\left(\mathrm{X} \subset \mathrm{X}^{\prime}\right)$, alors deux droites frontières, l'une de $\mathrm{X}$, l'autre de $\mathrm{X}^{\prime}$, n'ont pas d'intersection transverse.

Nous avons vu que le squelette de $\mathscr{D}$ pouvait être recouvert par des bonnes droites de Brouwer. La démonstration du théorème 1.1 est basée sur la proposition suivante, dont nous donnons une rapide preuve.

Proposition 3.1. - On peut recouvrir $\Sigma(\mathscr{D})$ par des bonnes droites de Brouwer n'ayant pas d'intersection transverse deux à deux. 
Démonstration. - Le plus petit bon attracteur contenant une brique $\beta$ est l'ensemble

$$
\mathrm{X}_{0}(\beta)=\bigcup_{n \geq 0}(\varphi \cup \psi)^{n}(\{\beta\}) .
$$

La proposition 2.13 nous dit que la relation

$$
\beta \leq \beta^{\prime} \text { si et seulement si } \beta \in \mathrm{X}_{0}\left(\beta^{\prime}\right)
$$

est antisymétrique. Comme elle est clairement réflexive et transitive, elle définit un ordre sur B. Il est facile de montrer, grâce au lemme de Zorn, que tout ordre sur un ensemble peut être prolongé en un ordre total (nous montrerons un résultat plus fort un peu plus loin). Prolongeons notre ordre en un ordre total noté de la même façon. Définissons ensuite, pour tout $\beta \in \mathrm{B}$, l'attracteur

$$
\mathrm{X}_{1}(\beta)=\bigcup_{\beta^{\prime} \leq \beta} \mathrm{X}_{0}\left(\beta^{\prime}\right) .
$$

La famille $\left(\mathrm{X}_{1}(\beta)\right)_{\beta \in \mathrm{B}}$ étant totalement ordonnée, l'ensemble des arcs frontières des $\mathrm{X}_{1}(\beta), \beta$ variant dans $\mathrm{B}$, est formé de droites de Brouwer n'ayant pas d'intersection transverse deux à deux. Montrons que ces droites recouvrent $\Sigma(\mathscr{D})$ et pour cela, montrons que toute arête $\alpha \in \mathrm{A}$ est incluse dans la frontière de $\partial \mathrm{X}_{1}(d(\alpha))$. Si ce n'est pas le cas, alors $g(\alpha) \in \mathrm{X}_{1}(d(\alpha))$. Il existe donc $\beta \leq d(\alpha)$ tel que $g(\alpha) \in \mathrm{X}_{0}(\beta)$, on a donc

$$
g(\alpha) \leq \beta \leq d(\alpha)<g(\alpha) .
$$

L'ensemble des droites de la proposition 3.1 n'a aucune raison d'être invariant sous l'action de G. En effet, si l'ordre partiel $\leq$ défini au début de la démonstration est équivariant, ce n'est pas nécessairement le cas du prolongement. On peut cependant choisir le prolongement équivariant si G est abélien. Le groupe $\mathrm{G}$ est nécessairement isomorphe à $\mathbf{Z}$ ou à $\mathbf{Z}^{2}$ et la surface $\mathbf{M}$ homéomorphe à l'anneau ouvert $\mathbf{T}^{1} \times \mathbf{R}$ ou au tore $\mathbf{T}^{2}$. Nous montrerons donc dans cette section :

Proposition 3.2. - Si $\mathrm{G}$ est abélien, on peut recouvrir $\Sigma(\mathscr{D})$ par une famille, invariante sous l'action de $\mathrm{G}$, de bonnes $\mathscr{D}$-droites de Brouwer n'ayant pas d'intersection transverse deux à deux.

C'est une conséquence immédiate du lemme ensembliste suivant, probablement très classique, et de la démonstration de la proposition 3.1.

Lemme 3.3. - Soit $\mathrm{G}$ un groupe abélien opérant sur un ensemble $\mathrm{X}$. On suppose que

$$
\mathrm{T} x \neq x \Longrightarrow \mathrm{T}^{k} x \neq x \text {, pour tout } k \in \mathbf{Z} \backslash\{0\} \text {. }
$$


On se donne un ordre équivariant sur $\mathrm{X}$, c'est à dire un ordre qui vérifie :

$$
x \leq x^{\prime} \Longrightarrow \mathrm{T} x \leq \mathrm{T} x^{\prime}, \text { pour tout } \mathrm{T} \in \mathrm{G}
$$

Il existe alors un ordre total équivariant qui prolonge $\leq$.

Démonstration. - On dit qu'une relation $\mathscr{R}_{1}$ sur $\mathrm{X}$ est moins fine qu'une relation $\mathscr{R}_{2}$ si

$$
x \mathscr{R}_{2} x^{\prime} \Longrightarrow x \mathscr{R}_{1} x^{\prime}, \text { pour tout } x \in \mathrm{X} \text { et } x^{\prime} \in \mathrm{X}
$$

On écrit $\mathscr{R}_{2} \preceq \mathscr{R}_{1}$ et on obtient un ordre sur l'ensemble des relations. Il est facile de voir que l'ensemble des ordres équivariants est inductif et par le lemme de Zorn, il suffit donc de montrer que tout ordre équivariant maximal est total.

Considérons un ordre équivariant $\leq$ et deux éléments $a$ et $b$ distincts de $\mathrm{X}$. On obtient une relation équivariante $\mathscr{R}_{1}$ moins fine que $\leq$ en posant :

$x \mathscr{R}_{1} x^{\prime}$ si $x \leq x^{\prime}$ ou s'il existe un entier $n \geq 1$, une suite $\left(x_{i}\right)_{0 \leq i \leq n}$, avec $x_{0}=x$, $x_{n}=x^{\prime}$, et une suite $\left(\mathrm{T}_{i}\right)_{0 \leq i<n}$ dans $\mathrm{G}$, telles que $x_{i} \leq \mathrm{T}_{i} a$ et $\mathrm{T}_{i} b \leq x_{i+1}$, pour tout $i \in\{0, \ldots, n-1\}$.

Cette relation est clairement équivariante, reflexive et transitive et on a $a \mathscr{R}_{1} b$. On définit de même une relation équivariante, réflexive, transitive, moins fine que $\leq$ et vérifiant $b \mathscr{R}_{2} a$ en intervertissant $a$ et $b$ dans la définition de $\mathscr{R}_{1}$. Il reste à montrer que l'une au moins des deux relations $\mathscr{R}_{1}$ et $\mathscr{R}_{2}$ est antisymétrique pour obtenir le lemme.

On raisonne par l'absurde, en notant $1_{\mathrm{G}}$ l'élément neutre de G. Si $\mathscr{R}_{1}$ n'est pas antisymétrique, on vérifie aisément qu'il existe une suite $\left(x_{i}\right)_{i \in \mathbf{Z} / n \mathbf{Z}}$ dans $\mathbf{X}$ et

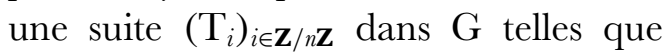

$$
x_{i} \leq \mathrm{T}_{i} a, \quad \mathrm{~T}_{i} b \leq x_{i+1} .
$$

De même, si $\mathscr{R}_{2}$ n'est pas antisymétrique, il existe une suite $\left(x_{i^{\prime}}^{\prime}\right)_{i^{\prime} \in \mathbf{Z} / n^{\prime} \mathbf{Z}}$ et une suite $\left(\mathrm{T}_{i^{\prime}}^{\prime}\right)_{i^{\prime} \in \mathbf{Z} / n^{\prime} \mathbf{Z}}$ telles que

$$
x_{i^{\prime}}^{\prime} \leq \mathrm{T}_{i^{\prime}}^{\prime} b, \quad \mathrm{~T}_{i^{\prime}}^{\prime} a \leq x_{i^{\prime}+1}^{\prime} .
$$

En posant

$$
\mathrm{R}_{i}=\mathrm{T}_{i}^{-1} \mathrm{~T}_{i+1}, \quad \mathrm{R}_{i}^{\prime}=\mathrm{T}_{i^{\prime}}^{-1} \mathrm{~T}_{i^{\prime}+1}^{\prime}
$$

on obtient

$$
b \leq \mathrm{R}_{i} a, \quad a \leq \mathrm{R}_{i^{\prime}}^{\prime} b,
$$


donc

$$
b \leq \mathrm{R}_{i} \mathrm{R}_{i^{\prime}}^{\prime} b
$$

pour tout choix de $i$ et $i^{\prime}$.

De la relation

$$
\prod_{1 \leq i \leq n} \mathrm{R}_{i}=1_{\mathrm{G}}
$$

et de la commutativité de $\mathrm{G}$, on déduit que pour toute famille $\left(i_{i}^{\prime}\right)_{i \in \mathbf{Z} / n \mathbf{Z}}$ dans $\mathbf{Z} / n^{\prime} \mathbf{Z}$, on a

$$
b \leq \prod_{1 \leq i \leq n} \mathrm{R}_{i_{i}^{\prime}}^{\prime} b .
$$

En particulier, pour tout $i^{\prime} \in \mathbf{Z} / n^{\prime} \mathbf{Z}$, on a

$$
b \leq\left(\mathbf{R}_{i^{\prime}}^{\prime}\right)^{n} b .
$$

On obtient donc, en fixant $i_{0}^{\prime} \in \mathbf{Z} / n^{\prime} \mathbf{Z}$ :

$$
\begin{aligned}
& b \leq\left(\mathrm{R}_{i_{0}^{\prime}}^{\prime}\right)^{n} b \\
& b \leq \prod_{i^{\prime} \neq i_{0}^{\prime}}\left(\mathrm{R}_{i^{\prime}}^{\prime}\right)^{n} b=\left(\mathrm{R}_{i_{0}^{\prime}}^{\prime}\right)^{-n} b,
\end{aligned}
$$

puis

$$
b=\left(\mathrm{R}_{i_{0}^{\prime}}^{\prime}\right)^{n} b .
$$

L'hypothèse faite sur $\mathrm{G}$ permet d'en déduire l'égalité $b=\mathrm{R}_{i_{0}^{\prime}}^{\prime} b$. L'inégalité $a \leq \mathrm{R}_{i_{0}^{\prime}}^{\prime}$, établie plus haut, devient $a \leq b$. Le rôle symétrique joué par $a$ et $b$ nous donne également $b \leq a$, ce qui contredit le caractère distinct de $a$ et $b$.

On peut donner une interprétation moins abstraite de la démonstration de la proposition 3.1 dans le cas où $G=\mathbf{Z}$ ou $G=\mathbf{Z}^{2}$.

Plaçons nous dans le premier cas et écrivons $\mathrm{G}=\left\{\mathrm{T}^{k}, k \in \mathbf{Z}\right\}$. L'ordre $\leq$ définit naturellement un préordre $\underset{\leq}{\widetilde{s}}$ sur $\widetilde{\mathrm{B}}$, c'est-à-dire une relation reflexive et transitive, de la façon suivante :

$$
\begin{aligned}
& \widetilde{\beta} \underset{\leq}{\beta^{\prime}} \text { si } \widetilde{\beta}=\widetilde{\beta}^{\prime} \text { ou s'il existe } \beta \in \pi^{-1}(\widetilde{\beta}) \text { et } \beta^{\prime} \in \pi^{-1}\left(\widetilde{\beta}^{\prime}\right) \text {, } \\
& \text { tels que } \beta \leq \beta^{\prime} \text {. }
\end{aligned}
$$

A notre préordre (comme à tout préordre) est naturellement associé une relation d'équivalence : $\beta \sim \beta^{\prime}$ si $\beta \widetilde{\leq} \beta^{\prime}$ et $\beta^{\prime} \underset{\leq}{\widetilde{x}} \beta$. L'ordre naturellement défini sur 
l'ensemble des classes d'équivalence de ce préordre est un ordre partiel que l'on prolonge en un ordre total. Pour obtenir un prolongement total équivariant sur $\mathrm{B}$ de l'ordre $\leq$, il suffit de trouver, pour toute classe d'équivalence $\widetilde{\mathrm{X}}$, un ordre équivariant sur $\mathrm{X}=\pi^{-1}(\tilde{\mathrm{X}})$.

L'ordre $\leq$ restreint à $\mathrm{X}$ peut se réduire à la relation d'égalité. Il y a alors une seule brique dans $\widetilde{\mathrm{X}}$ et on peut écrire $\mathrm{X}=\left\{\mathrm{T}^{k} \beta, k \in \mathbf{Z}\right\}$, où $\beta \in \mathrm{B}$. On munit cet ensemble de l'ordre trivial si $\mathrm{T} \beta=\beta$, de l'ordre induit naturellement par l'ordre usuel de $\mathbf{Z}$ si $\mathrm{T} \beta \neq \beta$ ou de l'ordre inverse (le choix est arbitraire). Si l'ordre sur $\mathrm{X}$ ne se réduit pas à la relation d'égalité, alors pour toute brique $\beta \in \mathrm{X}$, l'ensemble

$$
\mathrm{Z}=\left\{k \in \mathbf{Z} \mid \beta<\mathrm{T}^{k} \beta\right\}
$$

est non vide et formé d'entiers ayant tous le même signe puisqu'on sait que l'ordre $\leq$ est équivariant. De plus ce signe est indépendant de $\beta$. On suppose, par exemple, que ce signe est positif. On indexe les éléments $\widetilde{\beta}_{n}$ de $\widetilde{\mathrm{X}}$ par $\mathbf{N}$ (ou par $\{1, \ldots, \mathrm{N}\}$ si $\mathrm{X}$ est fini), on construit alors par récurrence sur $n$ un ordre total $\leq_{n}$ sur $\mathrm{X}_{n}=\pi^{-1}\left(\widetilde{\beta}_{0}, \ldots, \widetilde{\beta}_{n}\right\}$, qui prolonge à la fois $\leq$ et $\leq_{n-1}$, de la façon suivante :

- pour tout $\beta \in \pi^{-1}\left(\widetilde{\beta}_{n}\right)$ et pour tout $k \geq 0$, on a $\beta \leq_{n} \mathrm{~T}^{k} \beta$;

- pour tout $\beta \in \mathrm{X}_{n-1}$ et tout $\beta^{\prime} \in \pi^{-1}\left(\widetilde{\beta}_{n}\right)$, on a $\beta \leq_{n} \beta^{\prime}$ s'il existe $\beta^{\prime \prime} \in \mathrm{X}_{n-1}$ tel que $\beta \leq_{n-1} \beta^{\prime \prime} \leq \beta^{\prime}$;

- pour tout $\beta \in \mathrm{X}_{n-1}$ et tout $\beta^{\prime} \in \pi^{-1}\left(\widetilde{\beta}_{n}\right)$, on a $\beta^{\prime} \leq_{n} \beta$ si pour tout $\beta^{\prime \prime} \in \mathrm{X}_{n-1}$, tel que $\beta^{\prime \prime} \leq \beta^{\prime}$, on a $\beta^{\prime \prime}<_{n-1} \beta$.

Cette relation est clairement équivariante, réflexive et antisymétrique, on vérifie sans problème qu'elle est transitive.

Plaçons nous maintenant dans le second cas où $G$ est isomorphe à $\mathbf{Z}^{2}$ et écrivons

$$
\mathrm{G}=\left\{\mathrm{T}_{k}, k \in \mathbf{Z}^{2}\right\} \approx \mathbf{Z}^{2} \text { avec } \mathrm{T}_{k}=\mathrm{T}_{\left(k_{1}, k_{2}\right)}=\mathrm{T}_{1}^{k_{1}} \circ \mathrm{T}_{2}^{k_{2}} .
$$

On définit de façon analogue un préodre $\underset{\leq}{\widetilde{x}}$ sur $\widetilde{\mathrm{B}}$, on étend l'ordre naturel sur les classes d'équivalence en un ordre total et on cherche à définir pour toute classe d'équivalence $\tilde{\mathrm{X}}$, un ordre équivariant sur $\mathrm{X}=\pi^{-1}(\tilde{\mathrm{X}})$. Si l'ordre $\leq$ restreint à $\mathrm{X}$ se réduit à la relation d'égalité, il y a alors une seule brique dans $\widetilde{\mathrm{X}}$ et on peut écrire $\mathrm{X}=\left\{\mathrm{T}_{k} \beta, k \in \mathbf{Z}^{2}\right\}$, où $\beta \in \mathrm{B}$. Si le stabilisateur $\mathrm{H}_{\beta}$ de $\beta$ est non trivial, on définit un ordre sur $\mathrm{X}$ à partir d'un ordre équivariant sur $\mathrm{G} / \mathrm{H}_{\beta}$. S'il est trivial, on choisit un ordre arbitraire équivariant sur $\mathbf{Z}^{2}$, par exemple l'ordre lexicographique usuel. Si l'ordre sur $\mathrm{X}$ ne se réduit pas à la relation d'égalité, alors pour toute brique $\beta \in \mathrm{X}$, l'ensemble

$$
\mathrm{Z}_{\beta}=\left\{\mathrm{T} \in \mathbf{Z}^{2} \mid \beta<\mathrm{T} \beta\right\}
$$


est non vide et l'enveloppe convexe de $Z_{\beta}$ dans $\mathbf{R}^{2}$ ne contient pas 0 dans son intérieur, puisqu'on sait que l'ordre $\leq$ est équivariant. Il existe alors un demiplan vectoriel fermé, non nécessairement unique, qui contient tous les ensembles $\mathrm{Z}_{\beta}, \beta \in \mathrm{X}$. On l'écrit $\mathrm{L}^{-1}\left(\left[0,+\infty[)\right.\right.$, où $\mathrm{L}: \mathbf{R}^{2} \rightarrow \mathbf{R}$ est linéaire. Si on peut choisir la forme irrationnelle, alors $\mathrm{Z}_{\beta}$ est dans le demi-plan ouvert $\mathrm{L}^{-1}(] 0,+\infty[)$, et on peut reprendre mot à mot la démonstration faite dans le cas de l'anneau en remplaçant l'ordre naturel sur les entiers par l'ordre $\leq_{\mathrm{L}}$ défini ainsi :

$$
\mathrm{T}_{k} \leq_{\mathrm{L}} \mathrm{T}_{k^{\prime}} \Longleftrightarrow \mathrm{L}(k) \leq \mathrm{L}\left(k^{\prime}\right)
$$

Si on ne peut pas choisir la forme irrationnelle, alors le demi-plan est uniquement défini et on peut toujours supposer, sans perte de généralités, que c’est le demiplan d'équation $y \leq 0$. On définit

$$
\mathrm{G}_{1}=\left\{\mathrm{T}_{1}^{k_{1}}, k_{1} \in \mathbf{Z}\right\}, \quad \mathrm{G}_{2}=\left\{\mathrm{T}_{2}^{k_{2}}, k_{2} \in \mathbf{Z}\right\} .
$$

Le groupe $\mathrm{G}_{2}$ agit naturellement sur l'anneau $\mathbf{R}^{2} / \mathrm{G}_{1}$. On considère l'homéomorphisme $\widehat{f}$ de $\mathbf{R}^{2} / \mathrm{G}_{1}$, relevé par $f$ et la décomposition en briques $\widehat{\mathscr{D}}$ relevée par $\mathscr{D}$. On note alors $\widehat{\mathrm{X}}$ l'ensemble des briques qui se relèvent en des briques de $\mathrm{X}$. On définit là encore un préordre naturel $\widehat{\leq}$ sur $\widehat{\mathrm{X}}$, et on sait que $\widehat{\beta} \leq \mathrm{T}_{2}^{k_{2}} \widehat{\beta} \Longrightarrow k_{2} \leq 0$. On peut donc, comme dans le cas de l'anneau, prolonger cet ordre en un ordre total qui est $\mathrm{G}_{2}$-équivariant. On relève alors cet ordre à $\mathrm{X}$ et on le prolonge en un ordre invariant à la fois par $\mathrm{T}_{1}$ et $\mathrm{T}_{2}$.

Le résultat fondamental de cet article est la version généralisée au cas non abélien de la proposition 3.2, à savoir :

Proposition 3.4. - On peut recouvrir $\Sigma(\mathscr{D})$ par une famille, invariante sous l'action de $\mathrm{G}$, de bonnes $\mathscr{D}$-droites de Brouwer n'ayant pas d'intersection transverse deux à deux.

La démonstration, qui sera donnée dans les sections 5, 6 et 7 ne se limitera pas à des manipulations ensemblistes, comme dans le cas abélien, mais utilisera également des arguments topologiques plus fins. Dans la prochaine section, nous verrons comment déduire le théorème 1.2 de ce résultat.

\section{Construction du feuilletage à partir de la proposition fondamentale}

Nous allons reprendre dans cette section la construction du feuilletage vérifiant la proposition 1.1, faite dans [LeC5] à partir de la proposition 3.1, et voir que ce feuilletage peut être choisi équivariant si on suppose démontrée la proposition 3.4. Dans [LeC5], on supposait (et on justifiait pourquoi) que toutes les 
briques $\beta$ étaient bornées. Cette propriété supplémentaire n’était pas nécessaire, les principaux arguments restant valables en son absence. Elle permettait cependant de simplifier la rédaction de la preuve. Ici, d'une part on ne pourra pas supposer que les briques sont bornées, d'autre part il faudra faire attention que l'équivariance soit bien conservée dans la construction du feuilletage. L'explication détaillée de ce qui suit se trouve dans [LeG5].

Munissons A de la topologie discrète, puis $\mathrm{A}^{\mathbf{Z}}$ de la topologie produit et considérons le décalage

$$
\begin{aligned}
& \delta: \mathrm{A}^{\mathbf{Z}} \rightarrow \mathrm{A}^{\mathbf{Z}} \\
& \left(\alpha_{i}\right)_{i \in \mathbf{Z}} \mapsto\left(\alpha_{i+1}\right)_{i \in \mathbf{Z}} .
\end{aligned}
$$

L'ensemble des suites admissibles $\left(\alpha_{i}\right)_{i \in \mathbf{Z}}$, telles que $\prod_{i \in \mathbf{Z}} \alpha_{i}$ est une $\mathscr{D}$-droite de Brouwer, est une partie fermée $\mathscr{A}$ de $\mathrm{A}^{\mathbf{Z}}$ invariante par $\delta$.

Notons $\mathscr{B}$ l'ensemble des (bonnes) $\mathscr{D}$-droites de Brouwer. L'application

$$
\begin{aligned}
\Pi: \mathscr{A} & \rightarrow \mathscr{B} \\
\left(\alpha_{i}\right)_{i \in \mathbf{Z}} & \mapsto \prod_{i \in \mathbf{Z}} \alpha_{i}
\end{aligned}
$$

passe au quotient et définit une bijection entre l'ensemble $\mathscr{A} / \delta$ des orbites de $\delta$ contenues dans $\mathscr{A}$ et $\mathscr{B}$. On peut donc définir une topologie naturelle sur $\mathscr{B}$ en transportant la topologie quotient de $\mathscr{A} / \delta$. Cette topologie est généralement non séparée. Les ensembles $\mathscr{B}_{\alpha}, \alpha \in \mathrm{A}$, où $\mathscr{B}_{\alpha}$ désigne l'ensemble des bonnes $\mathscr{D}$-droites de Brouwer contenant $\alpha$, forment une base de voisinages de $\mathscr{B}$, ils sont ouverts, compacts (et donc en particulier séparés), mais non nécessairement fermés. La partie de $\mathscr{B} \times \mathscr{B}$ formée des couples de bonnes droites de Brouwer n'ayant pas d'intersection transverse est fermée dans $\mathscr{B} \times \mathscr{B}$. Notons $\mathscr{B}^{*}$ l'ensemble des droites de Brouwer définie par la proposition 3.4. Quitte à remplacer $\mathscr{B}^{*}$ par son adhérence, on peut supposer que cet ensemble est fermé dans $\mathscr{B}$. Les ensembles $\mathscr{B}_{\alpha}^{*}=\mathscr{B}^{*} \cap \mathscr{B}_{\alpha}, \alpha \in \mathrm{A}$, ne sont pas nécessairement fermés, il sont par contre compacts, ouverts dans $\mathscr{B}^{*}$, et définissent une base de la topologie de $\mathscr{B}^{*}$.

On munit $\mathscr{B}^{*}$ de l'ordre suivant :

$$
\Gamma \preceq \Gamma^{\prime} \Longleftrightarrow \mathrm{D}(\Gamma) \subset \mathrm{D}\left(\Gamma^{\prime}\right)
$$

Cet ordre n'est généralement pas total, mais restreint à chaque $\mathscr{B}_{\alpha}^{*}$ il le devient. De plus la topologie de $\mathscr{B}_{\alpha}^{*}$ est la topologie de l'ordre. Pour toute arête $\alpha$, on définit

$$
\Gamma_{\alpha}^{-}=\min \mathscr{B}_{\alpha}^{*} \quad \text { et } \quad \Gamma_{\alpha}^{+}=\max \mathscr{B}_{\alpha}^{*}
$$


Les droites isolées à droite, c'est-à-dire telles qu'il n'existe pas de suite $\left(\Gamma_{n}\right)_{n \geq 0}$ avec $\Gamma_{n}>\Gamma$ convergeant vers $\Gamma$ sont les droites $\Gamma_{\alpha}^{+}$; de même les droites isolées à gauche sont les droites $\Gamma_{\alpha}^{-}$.

Soient $\alpha$ et $\alpha^{\prime}$ deux arêtes :

- si $g(\alpha)=g\left(\alpha^{\prime}\right)$, alors $\Gamma_{\alpha}^{+}=\Gamma_{\alpha^{\prime}}^{+}$;

- si $d(\alpha)=d\left(\alpha^{\prime}\right)$, alors $\Gamma_{\alpha}^{-}=\Gamma_{\alpha^{\prime}}^{-}$;

- si $g(\alpha)=d\left(\alpha^{\prime}\right)$, alors $\Gamma_{\alpha}^{+} \prec \Gamma_{\alpha^{\prime}}^{-}$et $] \Gamma_{\alpha}^{+}, \Gamma_{\alpha^{\prime}}^{-}[=\emptyset$.

Posons alors

$$
\begin{cases}\Gamma_{\beta}^{+}=\Gamma_{\alpha}^{-} & \text {si } d(\alpha)=\beta, \\ \Gamma_{\beta}^{-}=\Gamma_{\alpha}^{+} & \text {si } g(\alpha)=\beta .\end{cases}
$$

On a donc

$$
\left.\Gamma_{\beta}^{-} \prec \Gamma_{\beta}^{+} \text {et }\right] \Gamma_{\beta}^{-}, \Gamma_{\beta}^{+}[=\emptyset .
$$

On définit la relation d'équivalence suivante :

$$
\beta \sim \beta^{\prime} \Longleftrightarrow \Gamma_{\beta}^{+}=\Gamma_{\beta^{\prime}}^{+} \text {et } \Gamma_{\beta}^{-}=\Gamma_{\beta^{\prime}}^{-} \text {. }
$$

On vérifie que deux briques adjacentes ne sont jamais équivalentes. On a également :

$$
\beta \sim \beta^{\prime} \Longleftrightarrow \beta^{\prime} \in \mathrm{D}\left(\Gamma_{\beta}^{+}\right) \cap \mathrm{G}\left(\Gamma_{\beta}^{-}\right)
$$

Si $\beta \in \mathrm{B}$ est une bande, alors $\Gamma_{\beta}^{-}=\partial \mathrm{X}_{\beta}^{+}$et $\Gamma_{\beta}^{+}=\partial \mathrm{X}_{\beta}^{-}$. Ces deux droites ne s'intersectent pas et la classe d'équivalence de $\beta$ se réduit à $\beta$. Si $\beta$ est un disque ou un demi-plan, les droites $\Gamma_{\beta}^{+}$et $\Gamma_{\beta}^{-}$s'intersectent et ont donc au moins une arête commune, elles contiennent le but et la source de $\beta$, si $\beta$ a un but ou une source. L'arc $\gamma_{\beta}^{+}=\Gamma_{\beta}^{+} \cap \beta$ (resp. $\gamma_{\beta}^{-}=\Gamma_{\beta}^{-} \cap \beta$ ) est formé des arêtes $\alpha$ telles que $d(\alpha)=\beta$ (resp. $g(\alpha)=\beta$ ). La réunion des arêtes communes à $\Gamma_{\beta}^{+}$et $\Gamma_{\beta}^{-}$ et des briques équivalentes à $\beta$ est une partie fermée $\mathrm{C}_{\beta}$ du plan, appelée chaîne d'équivalence, ne dépendant que de la classe d'équivalence $\widehat{\beta}$ de $\beta$. Dans $\mathbf{R}^{2}$, on a l'égalité

$$
\mathrm{G}_{\beta}=\mathrm{D}\left(\Gamma_{\beta}^{+}\right) \cap \mathrm{G}\left(\Gamma_{\beta}^{-}\right) .
$$

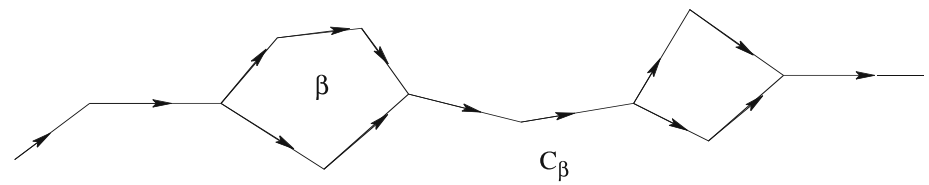


Tous les objets construits ici sont évidemment équivariants. Pour tout $\mathrm{T} \in \mathrm{G}$, on a :

$-\beta \sim \beta^{\prime} \Longleftrightarrow \mathrm{T} \beta \sim \mathrm{T} \beta^{\prime}$

$-\Gamma \preceq \Gamma^{\prime} \Longleftrightarrow \mathrm{T} \Gamma \preceq \mathrm{T} \Gamma^{\prime}$,

$-\Gamma_{\mathrm{T} \beta}^{+}=\mathrm{T} \Gamma_{\beta}^{+}$et $\Gamma_{\mathrm{T} \beta}^{-}=\mathrm{T} \Gamma_{\beta}^{-}$,

$-\mathrm{C}_{\mathrm{T} \beta}=\mathrm{TC}_{\beta}$.

On peut alors feuilleter chaque disque $\beta \in \mathrm{B}$ par une famille continue de segments $\left(\gamma_{\beta}^{t}\right)_{t \in[-1,1]}$ issus de $s(\beta)$ et aboutissant à $b(\beta)$; chaque demi-plan $\beta$ ayant une source en une famille continue de demi-droites positives $\left(\gamma_{\beta}^{t}\right)_{t \in[-1,1]}$ issues de $s(\beta)$, chaque demi-plan $\beta$ ayant un but en une famille continue de demi-droites négatives $\left(\gamma_{\beta}^{t}\right)_{t \in[-1,1]}$ aboutissant en $b(\beta)$, en demandant à chaque fois que $\gamma_{\beta}^{-1}=\gamma_{\beta}^{-}$ et $\gamma_{\beta}^{1}=\gamma_{\beta}^{+}$; chaque bande $\beta$ en une famille continue de droites $\left(\gamma_{\beta}^{t}\right)_{t \in[-1,1]}$, avec $\gamma_{\beta}^{-1}=\Gamma_{\beta}^{-}$et $\gamma_{\beta}^{1}=\Gamma_{\beta}^{+}$. Ces feuilletages peuvent être choisis équivariants : pour tout $\mathrm{T} \in \mathrm{G}$, on impose $\mathrm{T} \gamma_{\beta}^{t}=\gamma_{\mathrm{T} \beta}^{t}$, que le stabilisateur de $\beta$ soit trivial ou non.

Si $\beta$ est une bande, alors

$$
f(\beta) \subset \operatorname{Int}\left(\mathrm{D}\left(\Gamma_{\beta}^{-}\right)\right), \quad f^{-1}(\beta) \subset \operatorname{Int}\left(\mathrm{G}\left(\Gamma_{\beta}^{+}\right)\right),
$$

et donc, pour tout $t \in[0,1]$, on a

$$
f\left(\gamma_{\beta}^{t}\right) \subset \operatorname{Int}\left(\mathrm{D}\left(\Gamma_{\beta}^{-}\right)\right), \quad f^{-1}\left(\gamma_{\beta}^{t}\right) \subset \operatorname{Int}\left(\mathrm{G}\left(\Gamma_{\beta}^{+}\right)\right),
$$

chaque $\gamma_{\beta}^{t}$ est donc une droite de Brouwer. Si $\beta$ est un disque ou un demi-plan, on sait que

$$
f\left(\Gamma_{\beta}^{-}\right) \subset \operatorname{Int}\left(\mathrm{D}\left(\Gamma_{\beta}^{-}\right)\right), \quad f^{-1}\left(\Gamma_{\beta}^{+}\right) \subset \operatorname{Int}\left(\mathrm{G}\left(\Gamma_{\beta}^{+}\right)\right),
$$

on peut toujours construire la famille $\left(\gamma_{\beta}^{t}\right)_{t \in[-1,1]}$, de telle façon que :

$$
t \in[-1,-1 / 3] \Longrightarrow f\left(\gamma_{\beta}^{t}\right) \subset \operatorname{Int}\left(\mathrm{D}\left(\Gamma_{\beta}^{-}\right)\right)
$$

et

$$
t \in[1 / 3,1] \Longrightarrow f^{-1}\left(\gamma_{\beta}^{t}\right) \subset \operatorname{Int}\left(\mathrm{G}\left(\Gamma_{\beta}^{+}\right)\right)
$$

Pour toute classe d'équivalence $\widehat{\beta}$, écrivons $\Gamma_{\widehat{\beta}}^{-}=\Gamma_{\beta}^{-}$et $\Gamma_{\widehat{\beta}}^{+}=\Gamma_{\beta}^{+}$, où $\beta \in \widehat{\beta}$. Le stabilisateur $\mathrm{G}_{\widehat{\beta}}$ de la classe $\widehat{\beta}$ laisse invariant les droites $\Gamma_{\widehat{\beta}}^{-}$et $\Gamma_{\widehat{\beta}}^{+}$, il est donc trivial ou infini cyclique. L'ordre $\leq$ défini sur $\widehat{\beta}$ par la relation

$$
\beta \leq \beta^{\prime} \Longleftrightarrow \beta \in \bigcup_{n \geq 0}(\varphi \cup \psi)^{n}\left(\left\{\beta^{\prime}\right\}\right)
$$


se prolonge en un ordre total $\mathrm{G}_{\widehat{\beta}}$-équivariant d'après le lemme 3.3. Remarquons qu'on peut construire une famille $\left(\mu_{\beta}\right)_{\beta \in \widehat{\beta}}$ d'homéomorphismes croissants de $[-1,1]$ telle que

$$
\beta<\beta^{\prime} \Longrightarrow \mu_{\beta}\left(\frac{1}{3}\right)<\mu_{\beta^{\prime}}\left(-\frac{1}{3}\right) .
$$

En effet, on peut construire un isomorphisme $\beta \mapsto \mathrm{I}_{\beta}$ de l'ensemble ordonné $\widehat{\beta}$ dans un ensemble de segments disjoints de ]-1,1[, il suffit ensuite de supposer que $\mu_{\beta}$ envoie $[-1 / 3,1 / 3]$ sur $\mathrm{I}_{\beta}$. Dans le cas où $\mathrm{G}_{\widehat{\beta}}$ est non trivial et engendré par $\mathrm{T} \in \mathrm{G}^{*}$, on peut supposer, pour tout $\beta \in \widehat{\beta}$, que

$$
\mu_{\mathrm{T} \beta} \circ \mu_{\beta}^{-1}=\mu_{\mathrm{T}^{2} \beta} \circ \mu_{\mathrm{T} \beta}^{-1} .
$$

En effet $\mathrm{T}$ définit naturellement une bijection croissante sur l'ensemble des segments $\mathrm{I}_{\beta}$ et il existe un unique homéomorphisme $\tau$ de $[-1,1]$ qui envoie de façon affine tout segment $\mathrm{I}_{\beta}$ sur $\mathrm{I}_{\mathrm{T} \beta}$ et qui soit également affine sur toute composante connexe du complémentaire de la réunion des $\mathrm{I}_{\beta}$. On peut toujours choisir la famille $\mu_{\beta}$ pour avoir

$$
\mu_{\mathrm{T} \beta}=\tau \circ \mu_{\beta} .
$$

On peut vérifier alors (voir [LeC5]) que chaque droite

$$
\Gamma_{\widehat{\beta}}^{t}=\left(\Gamma_{\widehat{\beta}}^{-} \bigcap \Gamma_{\widehat{\beta}}^{+}\right) \bigcup_{\beta \in \widehat{\beta}} \gamma_{\beta}^{\mu_{\beta}^{-1} t},
$$

est une droite de Brouwer et que la famille obtenue est invariante par $\mathrm{G}_{\widehat{\beta}}$. Le groupe $G$ agit sur l'ensemble des classes d'équivalence et on peut supposer que la famille $\left(\Gamma_{\mathrm{T} \widehat{\beta}}^{t}\right)_{\mathrm{T} \in \mathrm{G}, t \in[0,1]}$ est invariante sous l'action de $\mathrm{G}$. La famille $\left(\Gamma_{\widehat{\beta}}^{t}\right)_{\widehat{\beta}, t}$ définit alors un quasi-feuilletage équivariant formé de droites de Brouwer. Plus précisément, cette famille est formée de droites de Brouwer n'ayant pas d'intersection transverse entre elles qui recouvrent le plan. Tout point $z \notin \Sigma(\mathscr{D})$ appartient à une unique droite et la famille définit un feuilletage au voisinage de $z$. Un point $z \in \Sigma(\mathscr{D}) \backslash \mathrm{S}$ peut appartenir à plusieurs droites, mais toutes ces droites coïncident au voisinage de $z$ avec l'unique arête contenant $z$, la famille définit encore un feuilletage au voisinage de $z$.

En reprenant mot à mot ce qui est fait dans [LeC5], on peut construire, en éclatant le quasi-feuilletage au voisinage de chaque sommet $\sigma \in \mathrm{S}$, un feuilletage équivariant $\mathscr{F}$ du plan en droites de Brouwer, tel que $\mathscr{B}^{*}$ soit isomorphe (du point de vue topologique et du point de vue de l'ordre) à une partie fermée de $\mathscr{F}$. La construction faite dans [LeC5] préserve l'équivariance. 


\section{Arcs de Brouwer presque simples}

Soit $\mathrm{H}$ un sous-groupe de G. On dira qu'un $\mathscr{D}$-arc bien orienté est H-simple si $\mathrm{T} \Gamma \cap \Gamma=\emptyset$, pour tout $\mathrm{T} \in \mathrm{H}$, et qu'il est $\mathrm{H}$-presque simple si $\mathrm{T} \Gamma$ et $\Gamma$ n'ont pas d'intersection transverse, pour tout $\mathrm{T} \in \mathrm{H}$. Un arc simple $\Gamma$ est $\mathrm{G}$-simple si et seulement si $\widetilde{\Gamma}=\pi \circ \Gamma$ est un arc simple dans $\mathrm{M}$.

On appellera bon $\mathscr{D}$-arc de Brouwer un $\mathscr{D}$-arc $\Gamma$ qui peut être prolongé en une bonne $\mathscr{D}$-droite de Brouwer. Une définition équivalente est de dire que $\Gamma$ est bien orienté et que $g(\Gamma)$ est disjoint de $\bigcup_{n \geq 0}(\varphi \cup \psi)^{n}(d(\Gamma))$ dans B. La condition est clairement nécessaire. Elle est également suffisante, car la droite frontière du bon attracteur $\bigcup_{n \geq 0}(\varphi \cup \psi)^{n}(d(\Gamma))$ qui contient $\Gamma$ est une bonne $\mathscr{D}$-droite de Brouwer. Dans le cas où $\Gamma$ est une $\mathscr{D}$-demi-droite, on parlera de bonne $\mathscr{D}$-demi-droite de Brouwer.

Les droites que l'on veut construire dans la proposition 3.4 sont toutes G-presque simples. Dans le but de prouver ce résultat, il est naturel de chercher déjà à construire une bonne $\mathscr{D}$-droite de Brouwer qui est G-presque simple. Remarquons que toute arête (et même tout arc bien orienté formé de deux arêtes) est un bon $\mathscr{D}$-arc de Brouwer G-presque simple. On peut donc se poser la question suivante :

Peut-on prolonger un bon $\mathscr{D}$-arc de Brouwer $\mathrm{G}$-presque simple $\Gamma$ en une bonne $\mathscr{D}$-droite de Brouwer G-presque simple?

Ce problème est difficile dans le cas où $\Gamma$ est un segment. Nous verrons dans cette section que la réponse est oui si $\Gamma$ est une demi-droite. C'est ce que dit la proposition suivante dans le cas particulier où $\mathrm{X}_{-}=\emptyset, \mathrm{X}_{+}=\mathrm{B}$ et $\mathrm{H}=\mathrm{G}$.

Proposition 5.1. - Soient $\mathrm{X}_{-} \subset \mathrm{X}_{+} \subset \mathrm{B}$ deux bons attracteurs invariants sous l'action d'un sous-groupe $\mathrm{H}$ de $\mathrm{G}$. Posons $\mathrm{K}=\mathrm{X}_{+} \backslash \operatorname{Int}\left(\mathrm{X}_{-}\right) \subset \mathbf{R}^{2}$. Toute bonne $\mathscr{D}$-demi-droite de Brouwer $\Gamma \subset \mathrm{K}$ qui est $\mathrm{H}$-presque simple peut être prolongée en une bonne $\mathscr{D}$-droite de Brouwer $\Gamma^{\prime} \subset \mathrm{K}$, également $\mathrm{H}$-presque simple.

Dans cette section, et jusqu'à la fin de la partie I de cet article, les seuls arcs que l'on rencontrera seront des $\mathscr{D}$-arcs, les droites de Brouwer seront de bonnes $\mathscr{D}$-droites de Brouwer, les attracteurs de bons attracteurs et les répulseurs de bons répulseurs. Nous omettrons donc les qualificatifs pour ne pas alourdir la rédaction.

Démonstration de la proposition 5.1. - On écrit $\Gamma=\prod_{m<i \leq 0} \alpha_{i}$, où $m \geq-\infty$. Il suffit de trouver une arête $\alpha_{1} \subset \mathrm{K}$, avec $s\left(\alpha_{1}\right)=b\left(\alpha_{0}\right)$, telle que $\prod_{m<i \leq 1} \alpha_{i}$ est 
une demi-droite (ou une droite) de Brouwer H-presque simple. En effet, en réitérant le processus, soit on obtient en temps fini une droite de Brouwer vérifiant la conclusion de la proposition, soit on construit une suite admissible $\left(\alpha_{i}\right)_{i \geq 1}$ de segments inclus dans $\mathrm{K}$, telle que chaque arc $\prod_{m<i \leq l} \alpha_{i}$ est une demi-droite de Brouwer H-presque simple. La droite $\prod_{m<i<+\infty} \alpha_{i}$ est alors une droite de Brouwer, puisque pour tous entiers $i$ et $j$, on a

$$
g\left(\alpha_{i}\right) \notin \bigcup_{n \geq 0}(\varphi \cup \psi)^{n}\left(d\left(\alpha_{j}\right)\right) .
$$

Elle est clairement $\mathrm{H}$-presque simple et vérifie la conclusion de la proposition.

Puisque $\Gamma$ est inclus dans $\mathrm{K}=\mathrm{X}_{+} \backslash \operatorname{Int}\left(\mathrm{X}_{-}\right)$et puisque $\mathrm{X}_{+}$et $\mathrm{X}_{-}$sont des attracteurs, on sait que

$$
d(\Gamma) \subset \mathrm{X}_{+}, \quad g(\Gamma) \cap \mathrm{X}_{-}=\emptyset .
$$

On sait également que

$$
\mathrm{X}=\mathrm{X}_{-} \bigcup_{n \geq 0}(\varphi \cup \psi)^{n}(d(\Gamma))
$$

est un attracteur compris entre $\mathrm{X}_{-}$et $\mathrm{X}_{+}$et que sa frontière contient $\Gamma$. L'arc frontière de $\mathrm{X}$ contenant $\Gamma$ est une droite de Brouwer, notée $\Gamma_{\text {prol }}$, contenue dans $\mathrm{K}$.

On écrira

$$
\mathrm{H}^{\prime}=\left\{\mathrm{T} \in \mathrm{H}^{*} \mid \alpha_{0} \subset \mathrm{T} \Gamma\right\} .
$$

Puisque $\Gamma$ est une demi-droite, $\alpha_{0}$ est un segment ou une demi-droite et on a $\mathrm{T} \alpha_{0} \neq \alpha_{0}$ si $\mathrm{T} \in \mathrm{H}^{*}$. On en déduit que tout arc $\mathrm{T} \Gamma, \mathrm{T} \in \mathrm{H}^{\prime}$, contient une arête $\alpha \subset \mathrm{K}$ de source $b\left(\alpha_{0}\right)$. S'il n'existe qu'une arête $\alpha_{1} \subset \mathrm{K}$ de source $b\left(\alpha_{0}\right)$, elle est nécessairement contenue dans $\Gamma_{\text {prol }}$ et l'arc $\prod_{m<i \leq 1} \alpha_{i}$ est H-presque simple. En effet, nous savons d'une part que tout translaté par $\mathrm{T} \in \mathrm{H}^{*}$ de cet arc, qui contient $\alpha_{0}$, contient également $\alpha_{1}$ et d'autre part que $\mathrm{T} \Gamma$ et $\Gamma$ n'ont pas d'intersection transverse.

Le cas non trivial est le cas où il existe deux arêtes $\alpha_{-}$et $\alpha_{+}$dans $\mathrm{K}$ de source $b\left(\alpha_{0}\right)$. On supposera alors que $d\left(\alpha_{+}\right)=g\left(\alpha_{-}\right)$. On note alors $\Gamma_{-}\left(\operatorname{resp} . \Gamma_{+}\right)$ le prolongement de $\Gamma$ par $\alpha_{-}$(resp. $\left.\alpha_{+}\right)$. Ces arcs sont inclus dans K. Tout arc $\mathrm{T} \Gamma, \mathrm{T} \in \mathrm{H}^{\prime}$, contient $\alpha_{-}$ou $\alpha_{+}$. On écrira alors

$$
\mathrm{H}^{\prime}=\mathrm{H}_{-} \sqcup \mathrm{H}_{+},
$$


où

$$
\mathrm{H}_{-}=\left\{\mathrm{T} \in \mathrm{H}^{\prime} \mid \alpha_{-} \subset \mathrm{T} \Gamma\right\} \quad \text { et } \quad \mathrm{H}_{+}=\left\{\mathrm{T} \in \mathrm{H}^{\prime} \mid \alpha_{+} \subset \mathrm{T} \Gamma\right\}
$$

On peut construire une autre décomposition

$$
\mathrm{H}^{\prime}=\mathrm{H}^{-} \sqcup \mathrm{H}^{+} \sqcup \mathrm{H}^{0}
$$

de la façon suivante :

- s'il existe $k \leq 0$ tel que ТГ contient le segment $\prod_{k \leq i \leq 0} \alpha_{i}$, ne contient pas l'arête $\alpha_{k-1}$ et contient une arête $\alpha \neq \alpha_{k-1}$ vérifiant

$$
b(\alpha)=s\left(\alpha_{k}\right), \quad d\left(\alpha_{k-1}\right)=g(\alpha),
$$

on dira que $\mathrm{T}$ appartient à $\mathrm{H}^{-}$;

- s'il existe $k \leq 0$ tel que Т $\Gamma$ contient le segment $\prod_{k \leq i \leq 0} \alpha_{i}$, ne contient pas l'arête $\alpha_{k-1}$ et contient une arête $\alpha \neq \alpha_{k-1}$ vérifiant

$$
b(\alpha)=s\left(\alpha_{k}\right), \quad g\left(\alpha_{k-1}\right)=d(\alpha),
$$

on dira que $\mathrm{T}$ appartient à $\mathrm{H}^{+}$;

- si $\Gamma \subset \mathrm{T} \Gamma$, on dira que $\mathrm{T}$ appartient à $\mathrm{H}^{0}$.

Le fait que, pour $T, T^{\prime} \in H$, les arcs $T \Gamma$ et $T^{\prime} \Gamma$ n'aient pas d'intersection transverse, nous dit que

$$
\mathrm{H}^{-} \cap \mathrm{H}_{+} \neq \emptyset \Longrightarrow \mathrm{H}^{+} \sqcup \mathrm{H}^{0} \subset \mathrm{H}_{+}
$$

et

$$
\mathrm{H}^{+} \cap \mathrm{H}_{-} \neq \emptyset \Longrightarrow \mathrm{H}^{-} \sqcup \mathrm{H}^{0} \subset \mathrm{H}_{-} .
$$

Dans le cas où

$$
\mathrm{H}^{-} \cap \mathrm{H}_{+}=\mathrm{H}^{+} \cap \mathrm{H}_{-}=\emptyset
$$

les arcs $\Gamma_{-}$et $\Gamma_{+}$sont H-presque simples, et l'un au moins d'entre eux (par exemple celui qui est inclus dans $\Gamma_{\text {prol }}$ ) est un arc de Brouwer, nous avons prolongé notre demi-droite $\Gamma$. Si $\mathrm{H}^{-} \cap \mathrm{H}_{+} \neq \emptyset$ (resp. $\mathrm{H}^{+} \cap \mathrm{H}_{-} \neq \emptyset$ ), alors $\Gamma_{+}$(resp. $\Gamma_{-}$) est H-presque simple, il reste à montrer que c'est aussi une demi-droite de Brouwer. Nous allons maintenant utiliser le fait important que $\Gamma$ est une demi-droite. 
Examinons le premier cas. Fixons $\mathrm{T} \in \mathrm{H}^{-} \cap \mathrm{H}_{+}$et notons $l<0$ l'entier tel que $\alpha_{0}=\mathrm{T} \alpha_{l}$. Il existe $k \leq 0$ tel que $\mathrm{T} \Gamma$ contient le segment

$$
\prod_{k \leq i \leq 0} \alpha_{i}=\prod_{k \leq i \leq 0} \mathrm{~T} \alpha_{i+l},
$$

et tel que

$$
b\left(\mathrm{~T} \alpha_{k+l-1}\right)=s\left(\alpha_{k}\right), \quad g\left(\mathrm{~T} \alpha_{k+l-1}\right)=d\left(\alpha_{k-1}\right) .
$$

La proposition 2.11 nous dit qu'il n'y a pas de $\mathscr{D}$-cercle bien orienté. On en conclut que toute arête $\mathrm{T} \alpha_{i}, i \leq k+l-1$, qui est incluse dans $\Gamma_{\text {prol }}$ est en fait incluse dans $\Gamma$ et même dans $\prod_{i<k} \alpha_{i}$. Les arêtes $T \alpha_{i}, k+l \leq i \leq l$, sont situées sur $\Gamma_{\text {prol }}$, contrairement à $T \alpha_{k+l-1}$ qui est cependant incluse dans $\mathrm{D}\left(\Gamma_{\text {prol }}\right)$. Montrons que toutes les arêtes $\mathrm{T} \alpha_{i}, i \leq l$, sont contenues dans $\mathrm{D}\left(\Gamma_{\text {prol }}\right)$. Dans le cas contraire, si on note $\mathrm{T} \alpha_{m}$ la première arête n'ayant pas cette propriété, l'arête $\mathrm{T} \alpha_{m+1}$ est sur $\Gamma_{\text {prol }}$ et il existe $n<k$ tel que

$$
\mathrm{T} \alpha_{m+1}=\alpha_{n+1}, \quad \mathrm{~T} \alpha_{m} \neq \alpha_{n}, \quad d\left(\mathrm{~T} \alpha_{m}\right)=g\left(\alpha_{n}\right) .
$$

Il existe alors un entier $r$ vérifiant

$$
2 \leq r \leq \min (k-n, k+l-1-m),
$$

tel que $\mathrm{T} \alpha_{m+i}=\alpha_{n+i}$ pour tout $i \in\{1, \ldots, r-1\}$, et

$$
\mathrm{T} \alpha_{m+r} \neq \alpha_{n+r}, \quad g\left(\mathrm{~T} \alpha_{m+r}\right)=d\left(\alpha_{n+r}\right) .
$$

Ceci contredit le fait que $\Gamma$ et $\mathrm{T} \Gamma$ n'ont pas d'intersection transverse.

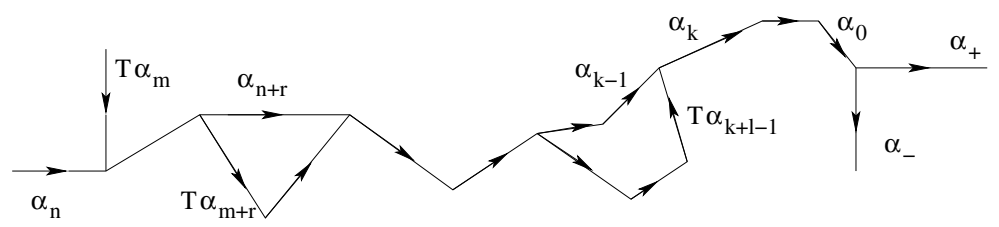

L'ensemble

$$
\mathrm{X}^{\prime}=\mathrm{D}\left(\Gamma_{\text {prol }}\right) \cup \mathrm{D}\left(\mathrm{T} \Gamma_{\text {prol }}\right)
$$

est un attracteur qui contient $d\left(\alpha_{0}\right)$ mais ne contient pas $g\left(\alpha_{0}\right)$. On peut donc considérer la composante connexe $\Gamma^{\prime}$ de $\partial \mathrm{X}^{\prime}$ qui contient $\alpha_{0}$. C'est une droite de Brouwer qui contient $\alpha_{+}$puisque $d\left(\alpha_{+}\right) \subset \mathrm{D}(\mathrm{T} \Gamma)$. On veut montrer qu'elle contient $\Gamma$. Si ce n'est pas le cas, il existe $k^{\prime} \leq 0$ tel que $\Gamma^{\prime}$ contient $\prod_{k^{\prime} \leq i \leq 0} \alpha_{i}$, ne contient pas $\alpha_{k^{\prime}-1}$ et contient une arête $\alpha \neq \alpha_{k^{\prime}-1}$ vérifiant $b(\alpha)=s\left(\alpha_{k^{\prime}}\right)$. Puisque $d\left(\alpha_{k^{\prime}-1}\right) \subset \mathrm{X}$, on sait que $d(\alpha)=g\left(\alpha_{k^{\prime}-1}\right)$, et puisque $\partial \mathrm{X} \subset \Gamma_{\text {prol }} \cup \Gamma_{\text {prol }}$, on sait que $\alpha$ appartient à $\mathrm{T} \Gamma_{\text {prol }}$. Comme il n'y a pas de $\mathscr{D}$-cercle bien orienté, $\alpha$ appartient donc à $\mathrm{T} \Gamma$, ce qui, on vient de le voir, est impossible. 


\section{Indices}

Nous fixons dans cette section un sous-groupe $H$ de $G$ et notons $\widehat{\mathscr{D}}=$ $(\widehat{\mathrm{S}}, \widehat{\mathrm{A}}, \widehat{\mathrm{B}})$ la décomposition en briques de la surface $\widehat{\mathrm{M}}=\mathrm{M} / \mathrm{H}$ relevée par $\mathscr{D}$. On dira, par définition, qu'une partie $\mathrm{Z} \subset \widehat{\mathrm{A}}$ est sans but (resp. sans source) si, pour tout $\alpha \in \mathrm{Z}$, il existe $\alpha^{\prime} \in \mathrm{Z}$ tel que $s\left(\alpha^{\prime}\right)=b(\alpha)$ (resp. $b\left(\alpha^{\prime}\right)=s(\alpha)$ ). On peut considérer là encore $\mathrm{Z}$ comme une partie fermée de $\widehat{\mathrm{M}}$. Nous allons montrer :

Proposition 6.1. - Soit $\mathrm{Z} \subset \widehat{\mathrm{A}}$ une partie sans but ou sans source et $\mathrm{Y}$ une composante connexe relativement compacte de $\widehat{\mathrm{M}} \backslash \mathrm{Z}$. La caractéristique d'Euler $\chi(\mathrm{Y})$ de $\mathrm{Y}$ est alors positive ou nulle, $\mathrm{Y}$ est un disque ouvert ou un anneau ouvert.

On donnera une preuve rigoureuse à la fin de la section. Commençons par une démonstration heuristique. Une partie $\mathrm{X} \subset \widehat{\mathrm{B}}$ est une variété à bord. On dira qu'un sommet $\sigma \in \widehat{\mathrm{S}} \cap \partial \mathrm{X}$ est un point de retournement de $\mathrm{X}$ si $\sigma$ est source ou but de deux arêtes situées sur $\partial \mathrm{X}$. Si la troisième arête d'extrémité $\sigma$ est incluse dans $\mathrm{X}$, on dira que $\sigma$ est un point de retournement intérieur de $\mathrm{X}$, sinon on dira que c'est un point de retournement extérieur. Remarquons que si la partie $\mathrm{X} \subset \widehat{\mathrm{M}}$ est compacte, alors

$$
\chi(\mathrm{X})=\frac{1}{2}\left(\sharp \operatorname{Ret}_{e}(\mathrm{X})-\sharp \operatorname{Ret}_{i}(\mathrm{X})\right),
$$

où on note $\operatorname{Ret}_{e}(\mathrm{X})$ (resp. $\left.\operatorname{Ret}_{i}(\mathrm{X})\right)$ l'ensemble des points de retournements extérieurs (resp. intérieurs). En effet, considérons l'ensemble stratifié $\Sigma(\widehat{\mathscr{D}}) \cap \mathrm{X}$ de $\mathrm{X}$. On obtient naturellement un ensemble stratifié sur le double $\mathrm{X}_{d}$ de $\mathrm{X}$ tel que :

- en tout sommet $\sigma \in \widehat{\mathrm{S}} \cap \partial \mathrm{X}$ correspondant à un retournement intérieur sont issues quatre arêtes ;

- en tout sommet $\sigma \in \widehat{\mathrm{S}} \cap \partial \mathrm{X}$ correspondant à un retournement extérieur sont issues deux arêtes ;

- en tout sommet $\sigma \in \widehat{\mathrm{S}} \cap \partial \mathrm{X}$ ne correspondant pas à un retournement sont issues deux ou quatre arêtes ;

- en tout sommet $\sigma \notin \widehat{\mathrm{S}} \cap \partial \mathrm{X}$ sont issues trois arêtes.

D'après les résultats de la section 2, on connait la topologie des briques de $\widehat{B}$ et l'orientation des arêtes sur leur frontière, on construit alors aisément un feuilletage singulier sur $\mathrm{X}_{d}$ dont les singularités sont les sommets de l'ensemble stratifié, tel que :

- tout sommet $\sigma \in \partial \mathrm{X}$ correspondant à un retournement intérieur est une singularité de type selle, d'indice -1 ;

- tout sommet $\sigma \in \partial \mathrm{X}$ correspondant à un retournement extérieur de type source est une source du feuilletage, d'indice 1 ; 
- tout sommet $\sigma \in \partial \mathrm{X}$ correspondant à un retournement extérieur de type but est un puits du feuilletage, d'indice 1 ;

- tout autre sommet est d'indice 0 .

L'entier

$$
\sharp \operatorname{Ret}_{e}(\mathrm{X})-\sharp \operatorname{Ret}_{i}(\mathrm{X})
$$

est alors égal à la caractéristique d'Euler de $\mathrm{X}_{d}$, c'est-à-dire au double de celle de X.

Étudions maintenant un cas plus général. Considérons une partie $Z \subset \widehat{A}$ et une partie relativement compacte $\mathrm{Y}$ de $\widehat{\mathrm{M}} \backslash \mathrm{Z}$. Pour toute arête orientée $\alpha \in \widehat{\mathrm{A}}$, notons $\alpha^{-1}$ l'arête munie de l'orientation inverse et écrivons

$$
b\left(\alpha^{-1}\right)=s(\alpha), \quad s\left(\alpha^{-1}\right)=b(\alpha), \quad g\left(\alpha^{-1}\right)=d(\alpha), \quad d\left(\alpha^{-1}\right)=g(\alpha) .
$$

Le bord de $\mathrm{Y}$ s'écrit comme réunion finie de lacets $\partial \mathrm{Y}=\bigcup_{0 \leq l \leq m} \Gamma_{l}$ et chaque lacet est un assemblage d'arêtes et s'écrit

$$
\Gamma_{l}=\prod_{i \in \mathbf{Z} / n ! \mathbf{Z}} \alpha_{l, i}^{\eta_{l, i}}
$$

où $\eta_{l, i} \in\{-1,1\}$, où $b\left(\alpha_{l, i}^{\eta_{l, i}}\right)=s\left(\alpha_{l, i+1}^{\eta_{l, i+1}}\right)$ et où $g\left(\alpha_{l, i}^{\eta_{l, i}}\right) \subset \mathrm{Y}$. On dira qu'on a un retournement en $(l, i)$ si $\eta_{l, i} \neq \eta_{l, i+1}$. Si $\eta_{l, i}=1$ et $\eta_{l, i+1}=-1$, on dira que le retournement est de type but; si $\eta_{l, i}=-1$ et $\eta_{l, i+1}=1$, on dira qu'il est de type source; si $g\left(\alpha_{l, i}^{\eta_{l, i}}\right)=g\left(\alpha_{l, i+1}^{\eta_{l, i+1}}\right)$, on dira qu'il est extérieur; si $d\left(\alpha_{l, i}^{\eta_{l, i}}\right)=d\left(\alpha_{l, i+1}^{\eta_{l, i+1}}\right)$, on dira qu'il est intérieur. Appelons indice de $\Gamma_{l}$ et notons $\mathrm{I}\left(\Gamma_{l}\right)$, la différence entre le nombre de retournements extérieurs et le nombre de retournements intérieurs en $(l, i)$, l'indice $i$ décrivant $\mathbf{Z} / n_{l} \mathbf{Z}$. On a démontré l'égalité

$$
\chi(\mathrm{Y})=\frac{1}{2} \sum_{l=0}^{m} \mathrm{I}\left(\Gamma_{l}\right)
$$

dans le cas où $\mathrm{Y}$ est l'intérieur d'une partie $\mathrm{X} \subset \widehat{\mathrm{A}}$. Pour montrer qu'elle est vraie dans le cas général, il suffit de découper la frontière de $\mathrm{Y}$ le long des lacets $\Gamma_{l}$ pour obtenir une variété à bord, construite abstraitement à partir de briques, où les arêtes peuvent apparaître deux fois, dont la caractéristique d'Euler est celle de $\mathrm{Y}$, un ensemble stratifié puis un feuilletage singulier sur le double de cette variété à bord.

Remarquons qu'il y a autant de retournements de type but que de retournements de type source. Si Z est sans but, alors tout retournement de type but est extérieur et correspond à un puits du feuilletage construit sur $\mathrm{Y}_{d}$, la somme 
$\sum_{l=0}^{m} \mathrm{I}\left(\Gamma_{l}\right)$ est donc positive ou nulle. S'il existe un retournement extérieur de type source la somme $\sum_{l=0}^{m} \mathrm{I}\left(\Gamma_{l}\right)$ est alors strictement positive. Puisque c'est la caractéristique d'Euler de $\mathrm{Y}$, cette somme est nécessairement égale à un, il y a alors exactement un retournement extérieur de type source. Si Z est sans but ni source, et s'il y a des retournements, la caractéristique d'Euler de Y est strictement positive. Elle est nécessairement égale à un, il y a alors un retournement de type source et un retournement de type but. On montrera donc :

Proposition 6.2. - Soit $\mathrm{Z} \subset \widehat{\mathrm{A}}$ une partie sans but et $\mathrm{Y}$ une composante connexe relativement compacte de $\widehat{\mathrm{M}} \backslash \mathrm{Z}$. Deux cas sont alors possibles :

- il n'y a pas de point de retournement extérieur de $\mathrm{Y}$ de type source et on a $\chi(\mathrm{Y})=0$, l'ensemble $\mathrm{Y}$ est un anneau ouvert ;

- il y a exactement un point de retournement extérieur de $\mathrm{Y}$ de type source et on a $\chi(\mathrm{Y})=1$, l'ensemble $\mathrm{Y}$ est un disque ouvert.

Proposition 6.3. - Soit $\mathrm{Z} \subset \widehat{\mathrm{A}}$ une partie sans but ni source et $\mathrm{Y}$ une composante connexe relativement compacte de $\widehat{\mathrm{M}} \backslash \mathrm{Z}$. Deux cas sont alors possibles :

- il n'y a pas de point de retournement de $\mathrm{Y}$ et on a $\chi(\mathrm{Y})=0$, l'ensemble $\mathrm{Y}$ est un anneau ouvert ;

- il y a un point de retournement extérieur de $\mathrm{Y}$ de type but, un point de retournement extérieur de type source et on a $\chi(\mathrm{Y})=1$, l'ensemble $\mathrm{Y}$ est un disque ouvert.

Nous allons donner une démonstration combinatoire plus rigoureuse des résultats précédents. L'ensemble $\widehat{\mathrm{B}}_{0}$ désignera l'ensemble des briques ouvertes de $\widehat{\mathscr{D}}$, qui, sont d'après la proposition 2.6 des disques ou des anneaux ouverts. De même $\widehat{\mathrm{A}}_{o}$ sera l'ensemble des arêtes ouvertes, c'est-à-dire privées de leurs extrémités. Nous noterons $\widehat{\mathrm{E}}=\widehat{\mathrm{S}} \sqcup \widehat{\mathrm{A}}_{0} \sqcup \widehat{\mathrm{B}}_{0}$ la réunion disjointe des éléments de la décomposition et là encore nous identifierons une partie $\mathrm{Y}$ de $\widehat{\mathrm{E}}$ et la partie $\mathrm{Y}=\bigcup_{\varepsilon \in \mathrm{Y}} \varepsilon$ de $\widehat{\mathrm{M}}$, ce qui nous permettra de qualifier une partie $\mathrm{Y} \subset \widehat{\mathrm{E}}$ de connexe, ouverte, fermée, compacte ou relativement compacte dans $\widehat{M}$. Remarquons qu'une partie relativement compacte est une partie finie dont toutes les briques et les arêtes sont relativement compacte, en particulier l'adhérence d'une arête est un segment ou un cercle.

Commençons par définir une application $\chi: \widehat{\mathrm{E}} \rightarrow \mathbf{Z}$ de la façon suivante :

- si $\varepsilon \in \widehat{\mathrm{S}}$, on pose $\chi(\varepsilon)=1$;

- si $\varepsilon \in \widehat{\mathrm{A}}_{o}$ est homéomorphe à ]0, 1[, on pose $\chi(\varepsilon)=-1$;

- si $\varepsilon \in \widehat{\mathrm{A}}_{o}$ est un cercle, on pose $\chi(\varepsilon)=0$;

- si $\varepsilon \in \widehat{\mathrm{B}}_{o}$ est un disque ouvert, on pose $\chi(\varepsilon)=1$;

- si $\varepsilon \in \widehat{\mathrm{B}}_{0}$ est un anneau ouvert, on pose $\chi(\varepsilon)=0$. 
Nous pouvons étendre $\chi$ en une fonction additive sur les parties finies de $\widehat{\mathrm{E}}$, à valeurs entières, en posant

$$
\chi(\mathrm{Y})=\sum_{\varepsilon \in \mathrm{Y}} \chi(\varepsilon)
$$

Remarquons que si $\mathrm{Y}$ est une partie ouverte ou compacte, $\chi(\mathrm{Y})$ n'est rien d'autre que la caractéristique d'Euler de $\mathrm{Y}$.

Soit $\mathrm{Y} \subset \widehat{\mathrm{E}}$ et $\sigma \in \widehat{\mathrm{S}}$. Notons $\alpha_{1}, \alpha_{2}$ et $\alpha_{3}$ les arêtes (dont deux sont éventuellement confondues) dont $\sigma$ est une extrémité et $\beta$ la brique dont $\sigma$ est une source ou un but. On définit une fonction $i_{\mathrm{Y}}: \widehat{\mathrm{S}} \rightarrow \mathbf{Z}$ en notant $\mathbf{1}_{\mathrm{Y}}: \widehat{\mathrm{E}} \rightarrow\{0,1\}$ la fonction caractéristique de $\mathrm{Y}$ et en posant

$$
i_{\mathrm{Y}}(\sigma)=\mathbf{1}_{\mathrm{Y}}(\sigma)-\frac{1}{2} \sum_{i=1}^{3} \mathbf{1}_{\mathrm{Y}}\left(\alpha_{i}\right)+\frac{1}{2} \mathbf{1}_{\mathrm{Y}}(\beta) .
$$

Lemme 6.4. - Pour toute partie relativement compacte $\mathrm{Y}$ la somme $\sum_{\sigma \in \widehat{\mathrm{S}}} i_{\mathrm{Y}}(\sigma)$ est finie et on a

$$
\sum_{\sigma \in \widehat{\mathrm{S}}} i_{\mathrm{Y}}(\sigma)=\chi(\mathrm{Y})
$$

Démonstration. - Le lemme est trivialement vrai si $\mathrm{Y}$ se réduit à un sommet, à une arête relativement compacte homéomorphe à ]0, 1[ ou à une arête homéomorphe à $\mathbf{T}^{1}$. Elle est également vraie si $\mathrm{Y}$ se réduit à un disque relativement compact, puisque cette brique a un but et une source, et si $\mathrm{Y}$ se réduit à un anneau, puisqu'il n'y a alors ni but ni source. Puisque $i_{\mathrm{Y}_{1} \cup \mathrm{Y}_{2}}=i_{\mathrm{Y}_{1}}+i_{\mathrm{Y}_{2}}$, si $\mathrm{Y}_{1}$ et $\mathrm{Y}_{2}$ sont disjoints, et puisque $\chi$ est additive, le lemme suit.

Si Y est ouvert, alors

$$
\begin{aligned}
& \sigma \in \mathrm{Y} \Longrightarrow i_{\mathrm{Y}}(\sigma)=1-\frac{3}{2}+\frac{1}{2}=0, \\
& \sigma \notin \overline{\mathrm{Y}} \Longrightarrow i_{\mathrm{Y}}(\sigma)=0-0+0=0,
\end{aligned}
$$

ainsi

$$
\chi(\mathrm{Y})=\sum_{\sigma \in \partial \mathrm{Y}} i_{\mathrm{Y}}(\sigma)
$$

Si $\sigma$ est un point isolé de $\partial \mathrm{Y}$, alors $i_{\mathrm{Y}}(\sigma)=-1$, sinon $i_{\mathrm{Y}}(\sigma)$ prend les valeurs $-1 / 2,1 / 2$ ou 0 . Nous avons dessiné ci-dessous les différents cas de figure, dans le cas où $\sigma$ est un sommet de type but (les éléments de $\mathrm{Y}$ sont les briques étoilées et les arêtes dessinées en pointillé). 

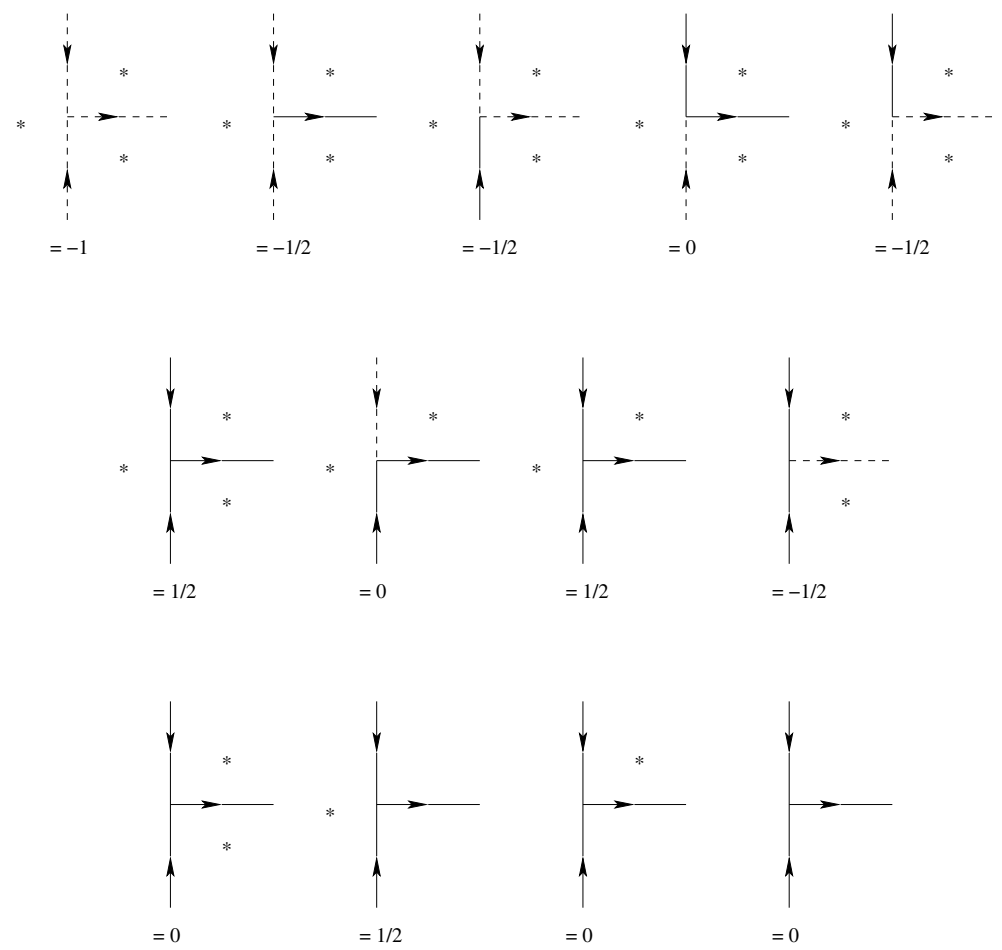

Démonstration de la proposition 6.1. - Supposons que Z soit sans but. Cet ensemble est une réunion de sommets et d'arêtes ouvertes et peut donc être assimilé à une partie de $\widehat{\mathrm{S}} \sqcup \widehat{\mathrm{A}}_{0}$. Remarquons d'abord qu'il n'y a pas de sommet isolé dans $\partial \mathrm{Y}$. Considérons la fonction

$$
n_{\mathrm{Y}}: \partial \mathrm{Y} \cap \widehat{\mathrm{A}}_{o} \rightarrow\{1,2\}
$$

qui, à une arête $\alpha \subset \partial \mathrm{Y}$, associe le nombre de briques adjacentes à $\alpha$ qui sont dans $\mathrm{Y}$, comptées avec leur multiplicité. En particulier $n_{\mathrm{Y}}(\alpha)=2$, s'il n'y a qu'une brique dans $\widehat{\mathrm{B}}_{o}$ qui est adjacente à $\alpha$. Considérons ensuite la fonction

$$
\begin{aligned}
m_{\mathrm{Y}}: \partial \mathrm{Y} \cap \widehat{\mathrm{S}} & \rightarrow \mathbf{Z} \\
s & \mapsto \sum_{i=1}^{3}(-1)^{\eta_{i}} n_{\mathrm{Y}}\left(\alpha_{i}\right)
\end{aligned}
$$

où $\alpha_{1}, \alpha_{2}$ et $\alpha_{3}$ sont les arêtes dans $\widehat{\mathrm{A}}_{0}$ d'extrémités $\sigma$, et où

$$
\begin{cases}\eta_{i}=1 & \text { si } \sigma=b\left(\alpha_{i}\right) \\ \eta_{i}=-1 & \text { si } \sigma=s\left(\alpha_{i}\right)\end{cases}
$$


Puisque toute arête $\alpha \subset \partial \mathrm{Y}$ a autant de buts que de sources, on a

$$
\sum_{\sigma \in \partial \mathrm{Y} \cap \widehat{\mathrm{S}}} m_{\mathrm{Y}}(\sigma)=0 .
$$

Pour montrer la proposition, il suffit donc de montrer que pour tout $\sigma \in \partial \mathrm{Y} \cap \widehat{\mathrm{S}}$, on a

$$
4 i_{\mathrm{Y}}(\sigma) \geq m_{\mathrm{Y}}(\sigma)
$$

Notons $\beta$ la brique dont $\sigma$ est une source ou un but et notons $n$ (resp. $m$ ) le nombre d'arêtes $\alpha_{1}, \alpha_{2}$ et $\alpha_{3}$ contenues dans $\partial \mathrm{Y}$ (resp. dans Y). Nous allons passer en revue les différents cas associés aux valeurs de $n, m$ et de $\mathbf{1}_{\mathrm{Y}}(\beta)$.

- Si $n=1$, alors $m=2$ et $\mathbf{1}_{\mathrm{Y}}(\beta)=1$. Il y a deux arêtes parmi $\alpha_{1}, \alpha_{2}$ et $\alpha_{3}$ qui sont dans $\mathrm{Y}$ et $\sigma$ est source de la troisième arête, puisque $\mathrm{Z}$ est sans but. On trouve

$$
i_{\mathrm{Y}}(\sigma)=-1 / 2, \quad m_{\mathrm{Y}}(\sigma)=-2 .
$$

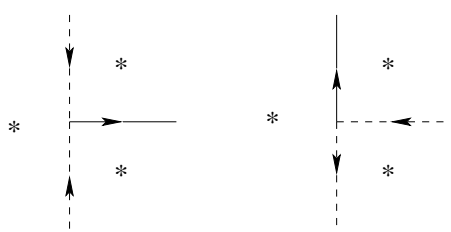

- Si $n=2, m=0$ et $\mathbf{1}_{\mathrm{Y}}(\beta)=0$, alors

$$
i_{\mathrm{Y}}(\sigma)=m_{\mathrm{Y}}(\sigma)=0
$$
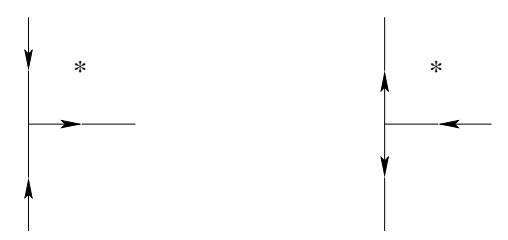

- Si $n=2, m=0$ et $\mathbf{1}_{\mathrm{Y}}(\beta)=1$, alors

$$
i_{\mathrm{Y}}(\sigma)=1 / 2, \quad m_{\mathrm{Y}}(\sigma)= \pm 2
$$

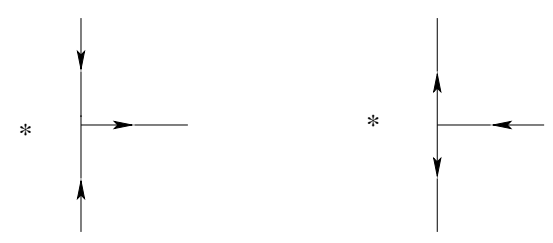


- Si $n=2, m=1$ et $\mathbf{1}_{\mathrm{Y}}(\beta)=0$, alors $\sigma=s(\beta)$ puisque $\mathrm{Z}$ est sans but. On trouve

$$
i_{\mathrm{Y}}(\sigma)=-1 / 2, \quad m_{\mathrm{Y}}(\sigma)=-2
$$

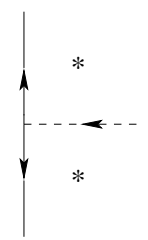

- Si $n=2, m=1$ et $\mathbf{1}_{\mathrm{Y}}(\beta)=1$, alors $i_{\mathrm{Y}}(\sigma)=0$. Si l'arête qui est dans $\mathrm{Y}$ est adjacente à $\beta$, alors $m_{\mathrm{Y}}(\sigma)=0$ qu'il y ait deux ou trois briques adjacentes à $\sigma$ qui soient dans $\mathrm{Y}$; sinon, puisque $\mathrm{Z}$ est sans but, $\sigma=s(\beta)$ et $m_{\mathrm{Y}}(\sigma)=-4$.

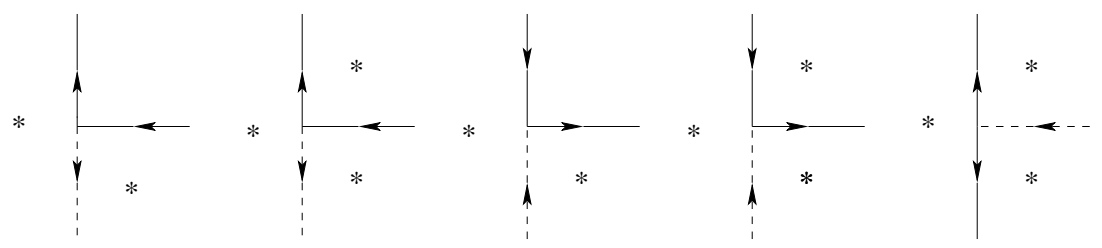

- Si $n=3$ et $\mathbf{1}_{\mathrm{Y}}(\beta)=0$, on trouve

$$
i_{\mathrm{Y}}(\sigma)=m_{\mathrm{Y}}(\sigma)=0
$$
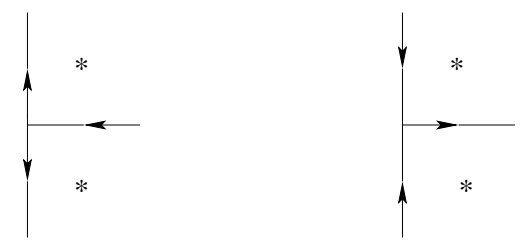

- Si $n=3$ et $\mathbf{1}_{\mathrm{Y}}(\beta)=1$, alors

$$
i_{\mathrm{Y}}(\sigma)=1 / 2, \quad m_{\mathrm{Y}}(\sigma)= \pm 2
$$

qu'il y ait deux ou trois briques adjacentes à $\sigma$ qui soient dans $\mathrm{Y}$.

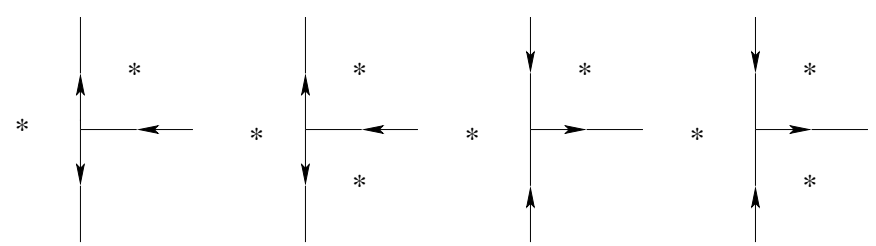


Si on garde les notations introduites au début de la section, on vérifie aisément que

$$
\sum_{\sigma \in \partial \mathrm{Y}} i_{\mathrm{Y}}(\sigma)=\frac{1}{2} \sum_{l=0}^{m} \mathrm{I}\left(\Gamma_{l}\right) .
$$

Démonstration de la proposition 6.2. - Pour comparer $m_{\mathrm{Y}}(\sigma)$ et $i_{\mathrm{Y}}(\sigma)$, nous avons distingué sept situations. On une inégalité stricte $4 i_{\mathrm{Y}}(\sigma)>m_{\mathrm{Y}}(\sigma)$ dans le deuxième sous-cas de la situation 3 ainsi que dans les deux premiers sous-cas de la situation 7. Ce sont les cas où apparaît un retournement extérieur de type source : il existe un sommet $\sigma \in \partial \mathrm{Y}$ qui est source d'une brique $\beta \in \mathrm{Y}$ tel qu'aucune des arêtes issues de $\sigma$ adjacentes à $\beta$ ne soit dans Y. S'il n'y a aucun retournement extérieur de type source, la caractéristique d'Euler $\chi(\mathrm{Y})$ de $\mathrm{Y}$ est nulle et $\mathrm{Y}$ est un anneau ouvert, sinon $\chi(\mathrm{Y})>0$. Dans ce cas, $\chi(\mathrm{Y})$ est nécessairement égal à un et $\mathrm{Y}$ est un disque ouvert. Il y a alors exactement un retournement extérieur de type source.

Démonstration de la proposition 6.3. - Supposons maintenant que Z soit sans but ni source. Pour comparer $m_{\mathrm{Y}}(\sigma)$ et $i_{\mathrm{Y}}(\sigma)$, nous avons distingué sept situations. Remarquons que les situations 1,4 sont interdites, de même que le cinquième sous-cas dans la situation 5. Si la situation 3 ou 7 a lieu, quitte à remplacer la fonction $m_{\mathrm{Y}}$ par son opposé, on va obtenir l'inégalité $\chi(\mathrm{Y})>0$. Ainsi si $\mathrm{Z}$ est sans but ni source, pour que $\chi(Y)$ soit nul, il faut que pour tout sommet $\sigma \in \partial Y$ qui soit but ou source d'une brique $\beta \in \mathrm{B}_{0}$, l'une des arêtes d'extremités $\sigma$ adjacente à $\beta$ soit dans Y. Dans le cas contraire, $\chi(\mathrm{Y})$ est nécessairement égal à un, puisque c'est la caractéristique d'Euler de Y, les situations 3 et 7 ne peuvent apparaître exactement que deux fois, une fois avec un sommet de type but, une autre fois avec un sommet de type source.

\section{Démonstration de la proposition 3.4}

L'ensemble constitué des parties $\mathscr{B}^{*} \subset \mathscr{B}$, invariantes sous l'action de $\mathrm{G}$ et formées de droites de Brouwer n'ayant pas d'intersections transverses deux à deux, est inductif pour l'inclusion et contient donc des éléments maximaux. On démontrera dans la proposition 7.3 que la réunion des droites d'une partie maximale $\mathscr{B}^{*}$ recouvre $\Sigma(\mathscr{D})$, ce qui prouvera la proposition 3.4 .

La proposition 3.4 implique que toute arête est contenue dans une droite de Brouwer G-presque simple. Il semble très difficile de montrer directement ce résultat, par contre nous allons prouver, dans la proposition qui suit, qu'il existe une droite de Brouwer qui est G-presque simple, ce qui signifie qu'une partie maxi- 
male $\mathscr{B}^{*}$ n'est pas vide. À vrai dire, ce résultat préliminaire n'est pas nécessaire à la preuve de la proposition 7.3. Il nous a semblé cependant utile de l'inclure car le principe de la démonstration y apparaît déjà et se comprend davantage dans ce cadre particulier.

\section{Proposition 7.1. - Il existe une droite de Brouwer G-presque simple.}

Démonstration. - Nous pouvons toujours supposer que le groupe G n'est pas abélien puisque le cas abélien a été traité dans la section 3. Fixons $z \in \mathbf{R}^{2}$ et deux éléments $\mathrm{T}$ et $\mathrm{T}^{\prime}$ de $\mathrm{G}$ ne commutant pas. On considère alors un arc $\gamma$ joignant $z$ à $\mathrm{T} z$ et un arc $\gamma^{\prime}$ joignant $z$ à $\mathrm{T}^{\prime} z$. Les lacets $\tilde{\gamma}=\pi \circ \gamma$ et $\tilde{\gamma}^{\prime}=\pi \circ \gamma^{\prime}$ définissent deux éléments du groupe fondamental $\pi_{1}(\mathrm{M}, \pi(z))$ qui ne commutent pas. On en déduit qu'il n'existe dans $M$, aucun disque ouvert, ni aucun anneau ouvert, qui contient $\widetilde{\gamma}$ et $\widetilde{\gamma}^{\prime}$. Rappelons que $\widetilde{\mathscr{D}}=(\widetilde{\mathrm{S}}, \widetilde{\mathrm{A}}, \widetilde{\mathrm{B}})$ est la décomposition de $\mathrm{M}$ relevée par $\mathscr{D}$. Grâce à la proposition 6.1 , on sait donc que pour toute partie $Z \subset \widetilde{A}$, qui est sans but ou sans source et qui est disjointe de $\tilde{\gamma}$ et de $\tilde{\gamma}^{\prime}$, la composante connexe $\mathrm{Y}$ de $\tilde{\mathrm{M}} \backslash \mathrm{Z}$ qui contient $\pi(z)$ n'est pas relativement compacte.

Grâce à la proposition 5.1, il suffit de construire une demi-droite G-presque simple. Toute arête $\alpha \in \mathrm{A}$ étant $\mathrm{G}$-presque simple, nous pouvons donc supposer que toutes les arêtes $\alpha \in \mathrm{A}$ sont des segments. Les arêtes $\alpha \in \widetilde{\mathrm{A}}$ sont alors toutes compactes. De même nous pouvons supposer que les briques $\beta \in \mathrm{B}$ sont des disques fermés, puisque la frontière d'une brique non compacte contient une demi-droite ou une droite de Brouwer qui est G-presque simple. Les briques $\beta \in \widetilde{\mathrm{B}}$ sont alors également toutes compactes. La surface $\mathrm{M}$ est différente de $\mathbf{T}^{2}$ puisque G n'est pas abélien. Expliquons pourquoi elle ne peut pas être compacte. Dans le cas contraire c'est une surface compacte de genre $\geq 2$ (puisque G n'est pas abélien) et sa caractéristique d'Euler est donc strictement négative. L'homéomorphisme $\widetilde{f}$ de $\mathrm{M}$ relevé par $f$ est isotope à l'identité et admet donc au moins un point fixe par la formule de Lefschetz. En fait, d'après la formule de Nielsen (voir Jiang [J] par exemple), il admet au moins un point fixe contractile (i.e. dont les antécédents dans $\mathbf{R}^{2}$ sont des point fixes de $f$ ), ce qui est bien sûr impossible. Nous rappelerons les formules de Lefschetz et de Nielsen en section 10. Nous savons donc que $\widetilde{\mathrm{B}}$ et $\widetilde{\mathrm{A}}$ sont infinis. On écrit

$$
\widetilde{\mathrm{A}}=\bigcup_{n \geq 0} \widetilde{\mathrm{A}}_{n},
$$

où $\left(\widetilde{\mathrm{A}}_{n}\right)_{n \geq 0}$ est une famille strictement croissante de parties finies.

Nous allons voir qu'il existe une demi-droite de Brouwer G-simple, c'est-àdire qu'il existe une demi-droite de $\widetilde{M}$ qui se relève en une famille de demi-droites 
de Brouwer. Fixons $\alpha \in \widetilde{\mathrm{A}}$ et choisissons une arête $\alpha^{\prime} \in \pi^{-1}(\alpha)$. L'arc frontière de $\bigcup_{n \geq 0}(\varphi \cup \psi)^{n}\left(d\left(\alpha^{\prime}\right)\right)$ qui contient $\alpha^{\prime}$ est une droite de Brouwer $\Gamma_{\alpha^{\prime}}=\prod_{i=-\infty}^{+\infty} \alpha_{i}^{\prime}$, où $\alpha_{0}^{\prime}=\alpha^{\prime}$. Notons $\Gamma_{\alpha^{\prime}}^{+}$la demi-droite positive incluse dans cet arc qui débute par $\alpha^{\prime}$. En notant $\alpha_{i}=\pi\left(\alpha_{i}^{\prime}\right)$, on peut définir

$$
\widetilde{\Gamma}_{\alpha}=\pi\left(\Gamma_{\alpha^{\prime}}\right)=\prod_{i=-\infty}^{+\infty} \alpha_{i}, \quad \widetilde{\Gamma}_{\alpha}^{+}=\pi\left(\Gamma_{\alpha^{\prime}}^{+}\right)=\prod_{i=0}^{+\infty} \alpha_{i} .
$$

L'arc $\widetilde{\Gamma}_{\alpha}^{+}$se relève en une famille de demi-droites de Brouwer. Si $\widetilde{\Gamma}_{\alpha}^{+}$est simple, nous pouvons conclure. Nous supposerons donc que pour tout $\alpha \in \widetilde{\mathrm{A}}$, l'arc $\widetilde{\Gamma}_{\alpha}^{+}$ n'est pas simple. Il existe alors deux entiers $i_{\alpha}$ et $i_{\alpha}^{\prime}$, avec $0 \leq i_{\alpha}^{\prime} \leq i_{\alpha}$, tels que $b\left(\alpha_{i_{\alpha}}\right)=s\left(\alpha_{i_{\alpha}^{\prime}}\right)$ et tels que l'arc $\prod_{i=0}^{i_{\alpha}} \alpha_{i}$ privé de l'extrémité $b\left(\alpha_{i_{\alpha}}\right)$ soit simple. On posera

$$
\tilde{\gamma}_{\alpha}=\prod_{i=0}^{i_{\alpha}} \alpha_{i}
$$

Pour tout $n \geq 0$, l'ensemble

$$
\mathrm{Z}_{n}=\bigcup_{\alpha \in \widetilde{A} \backslash \widetilde{\mathrm{A}}_{n}} \tilde{\gamma}_{\alpha}
$$

est sans but et toutes les composantes connexes de son complémentaire sont relativement compactes. Il existe donc une arête $\alpha_{n} \notin \widetilde{\mathrm{A}}_{n}$ telle que $\widetilde{\gamma}_{\alpha_{n}}$ rencontre $\tilde{\gamma} \cup \tilde{\gamma}^{\prime}$. Écrivons

$$
\widetilde{\gamma}_{\alpha_{n}}=\prod_{i=0}^{i_{n}} \alpha_{i}^{n}
$$

Notons $\widetilde{\mathrm{A}}_{*}$ l'ensemble (fini) des arêtes qui rencontrent $\tilde{\gamma} \cup \widetilde{\gamma}^{\prime}$, et $\widetilde{\mathrm{A}}_{* *}$ l'ensemble (également fini) des arêtes qui rencontrent au moins une arête de $\widetilde{\mathrm{A}}_{*}$. Pour tout $n \geq 0$, il existe $j_{n}<i_{n}$ tel $\alpha_{j_{n}}^{n} \in \widetilde{\mathrm{A}}_{* *}$ et on a bien évidemment $\lim _{n \rightarrow+\infty} j_{n}=$ $+\infty$. Puisque $\widetilde{\mathrm{A}}_{* *}$ est fini, on peut extraire une suite convergente de la suite $\left(\prod_{i=-\infty}^{j_{n}} \alpha_{i}^{n}\right)_{n \geq 0}$. La limite se relève en une famille de demi-droites négatives de Brouwer, c'est une demi-droite (i.e. elle est simple) puisque $\prod_{i=0}^{j_{n}} \alpha_{i}^{n}$ est simple et puisque $\lim _{n \rightarrow+\infty} j_{n}=+\infty$. 
Énonçons maintenant le lemme fondamental qui généralise la proposition 7.1 :

Lemme 7.2. - Soient $\mathrm{X}_{-}$et $\mathrm{X}_{+}$deux attracteurs, avec $\mathrm{X}_{-} \subset \mathrm{X}_{+} \subset \mathrm{B}$, invariants sous l'action d'un sous-groupe $\mathrm{H}$ de $\mathrm{G}$, et $\mathrm{K}=\mathrm{X}_{+} \backslash \operatorname{Int}\left(\mathrm{X}_{-}\right) \subset \mathbf{R}^{2}$. On suppose qu'il existe une arête $\alpha_{0}$ telle que $g\left(\alpha_{0}\right) \cup d\left(\alpha_{0}\right) \subset \mathrm{K}$. Il existe alors une droite de Brouwer $\Gamma \subset \mathrm{K}$, qui est $\mathrm{H}$-presque simple et qui contient une arête $\alpha$ telle que $g(\alpha) \cup d(\alpha) \subset \mathrm{K}$.

Démonstration. - On note $\mathrm{K}_{0}$ la composante connexe de $\mathrm{K}$ qui contient $\alpha_{0}$ et $\mathrm{H}_{0} \subset \mathrm{H}$ le stabilisateur dans $\mathrm{H}$ de $\mathrm{K}_{0}$. Par hypothèse, on sait qu'il existe au moins une composante connexe de $\mathrm{X}^{+} \backslash \mathrm{X}^{-}$dans $\mathrm{B}$ qui est non triviale c'est-à-dire qui ne se réduit pas à une brique. Nous allons étudier trois situations où le lemme peut être prouvé, nous verrons ensuite que l'une au moins de ces situations a lieu. Énonçons-les :

i) le groupe $\mathrm{H}_{0}$ est abélien ;

ii) il existe une composante non triviale $\mathrm{X}$ de $\mathrm{X}_{+} \backslash \mathrm{X}_{-}$(dans $\mathrm{B}$ ), incluse dans $\mathrm{K}_{0}$, dont le stabilisateur $\mathrm{H}_{1} \subset \mathrm{H}_{0}$ dans $\mathrm{H}_{0}$ est non abélien ;

iii) il existe une composante non triviale $\mathrm{X}$ de $\mathrm{X}_{+} \backslash \mathrm{X}_{-}$(dans $\mathrm{B}$ ) incluse dans $\mathrm{K}_{0}$, dont le stabilisateur $\mathrm{H}_{1} \subset \mathrm{H}_{0}$ dans $\mathrm{H}_{0}$ est abélien et non trivial.

Il suffit de construire une demi-droite de Brouwer dans $\mathrm{K}_{0}$ qui est $\mathrm{H}_{0^{-}}$ presque simple, et qui contient une arête $\alpha$ telle que $g(\alpha) \cup d(\alpha) \subset \mathrm{K}_{0}$. Puisqu'elle est disjointe de son image par tout $\mathrm{T} \in \mathrm{H} \backslash \mathrm{H}_{0}$, elle est en fait $\mathrm{H}$-presque simple et on peut la prolonger en une droite de Brouwer H-presque simple incluse dans $\mathrm{K}$ d'après la proposition 5.1 .

Cas i). - L'ensemble

$$
d\left(\mathrm{~K}_{0}\right)=\left\{\beta \in \mathrm{B} \mid \text { il existe } \alpha \subset \mathrm{K}_{0} \text { telle que } \beta=d(\alpha)\right\}
$$

est inclus dans $\mathrm{X}_{+}$et invariant sous l'action de $\mathrm{H}_{0}$. Rappelons l'ordre défini sur cet ensemble par :

$$
\beta^{\prime} \leq \beta \text { si } \beta^{\prime} \in \mathrm{X}_{0}(\beta)=\bigcup_{n \geq 0}(\varphi \cup \psi)^{n}(\{\beta\}) .
$$

Puisque $\mathrm{H}_{0}$ est abélien, on peut utiliser le lemme 3.3 pour prolonger cet ordre en un ordre total $\leq$ qui est $\mathrm{H}_{0}$-équivariant. On définit alors, pour toute brique $\beta \in d\left(\mathrm{~K}_{0}\right)$, l'attracteur

$$
\mathrm{X}_{1}(\beta)=\mathrm{X}_{-} \bigcup_{\beta^{\prime} \leq \beta} \mathrm{X}_{0}(\beta) .
$$


Puisque $\mathrm{X}_{-}$est invariant sous l'action de $\mathrm{H}_{0}$ et puisque $\leq$ est $\mathrm{H}_{0}$-équivariant, on a

$$
\mathrm{TX}_{1}(\beta)=\mathrm{X}_{1}(\mathrm{~T} \beta)
$$

pour tout $\beta \in d\left(\mathrm{~K}_{0}\right)$ et tout $\mathrm{T} \in \mathrm{H}_{0}$. Les attracteurs $\mathrm{X}_{0}(\beta), \beta \in d\left(\mathrm{~K}_{0}\right)$, étant inclus dans $\mathrm{X}_{+}$, on a

$$
\mathrm{X}_{-} \subset \mathrm{X}_{1}(\beta) \subset \mathrm{X}_{+}
$$

Pour toute arête $\alpha \in \mathrm{K}_{0}$, on a $d(\alpha) \in \mathrm{X}_{+}$et $g(\alpha) \notin \mathrm{X}_{-}$. On a vu, dans la démonstration de la proposition 3.1 que $g(\alpha) \notin \bigcup_{\beta \leq d(\alpha)} \mathrm{X}_{0}(\beta)$. On sait donc que la frontière de $\mathrm{X}_{1}(d(\alpha))$ contient $\alpha$. Puisque $\mathrm{X}_{-} \subset \mathrm{X}_{1}(d(\alpha)) \subset \mathrm{X}_{+}$, on sait de plus que l'arc frontière $\Gamma_{\alpha}$ qui contient $\alpha$ est inclus dans $\mathrm{K}_{0}$. Les $\mathrm{X}_{1}(d(\alpha)), \alpha \subset \mathrm{K}_{0}$, étant tous comparables, les droites $\Gamma_{\alpha}, \alpha \in \mathrm{K}_{0}$, sont des droites de Brouwer sans intersection transverse. Puisque $\mathrm{T} \Gamma(\alpha)=\Gamma(\mathrm{T} \alpha)$, si $\mathrm{T} \in \mathrm{H}_{0}$, elles sont $\mathrm{H}_{0}$-presque simples. La droite $\Gamma_{\alpha_{0}}$ convient.

Cas ii). - On va utiliser les mêmes arguments, légèrement modifiés, que dans la preuve de la proposition 7.1. Supposons que $\mathrm{X}$ soit une composante non triviale de $\mathrm{X}_{+} \backslash \mathrm{X}_{-}$incluse dans $\mathrm{K}_{0}$, dont le stabilisateur $\mathrm{H}_{1} \subset \mathrm{H}_{0}$ dans $\mathrm{H}_{0}$ est non abélien. Considérons le revêtement

$$
\widehat{\pi}: \mathbf{R}^{2} \rightarrow \widehat{\mathrm{M}}=\mathbf{R}^{2} / \mathrm{H}_{1}
$$

et la décomposition $\widehat{\mathscr{D}}=(\widehat{\mathrm{S}}, \widehat{\mathrm{A}}, \widehat{\mathrm{B}})$ de $\widehat{\mathrm{M}}$ relevée par $\mathscr{D}$. L'ensemble

$$
\mathrm{Z}=\widehat{\pi}\left(\partial \mathrm{X}_{-} \cup \partial \mathrm{X}_{+}\right)
$$

est sans but ni source et

$$
\mathrm{Y}=\widehat{\pi}(\operatorname{Int}(\mathrm{X}))
$$

est une composante connexe du complémentaire de Z. Posons

$$
\widehat{\mathrm{A}}_{\mathrm{Y}}=\{\alpha \in \widehat{\mathrm{A}} \mid \operatorname{int}(\alpha) \subset \mathrm{Y}\}
$$

et notons $\widehat{\mathrm{B}}_{\mathrm{Y}}$ l'ensemble des briques $\beta \in \widehat{\mathrm{B}}$ telles que la brique ouverte correspondante soit contenue dans $\mathrm{Y}$. S'il existe une bande $\beta \in \mathrm{X}$, les deux droites frontières de $\beta$ sont des droites de Brouwer G-presque simples contenues dans $\mathrm{K}_{0}$. L'une au moins de ces droites contient une arête $\alpha$ telle que $g(\alpha) \cup d(\alpha) \subset \mathrm{K}_{0}$, sinon $\mathrm{K}_{0}$ se réduit à la brique $\beta$ et son stabilisateur est trivial ou infini cyclique et donc abélien. De même, s'il existe un demi-plan $\beta \in \mathrm{X}$, les demi-droites $\gamma_{\beta}^{-}$et 
$\gamma_{\beta}^{+}$formées des arêtes $\alpha$ vérifiant respectivement $g(\alpha)=\beta$ et $d(\alpha)=\beta$, sont des demi-droites de Brouwer G-presque simples et contenues dans $\mathrm{K}_{0}$. Là encore, l'une au moins de ces demi-droites contient une arête $\alpha$ telle que $g(\alpha) \cup d(\alpha) \subset \mathrm{K}_{0}$. On supposera donc que toutes les briques $\beta \in \mathrm{X}$ sont des disques et que les briques $\beta \in \widehat{\mathrm{B}}_{\mathrm{Y}}$ sont donc compactes. Les arêtes $\alpha \in \widehat{\mathrm{A}}_{\mathrm{Y}}$ sont également compactes et leur antécédents par $\widehat{\pi}$ sont des segments. On considère, comme dans la preuve de la proposition 7.1, deux lacets $\widehat{\gamma}$ et $\widehat{\gamma}^{\prime}$, basés en un point $\widehat{z} \in \mathrm{Y}$, définissant deux éléments du groupe fondamental $\pi_{1}(\mathrm{Y}, \widehat{z})$ qui ne commutent pas. Puisque $\mathrm{Z}$ est sans but ni source et puisque la caractéristique d'Euler de $\mathrm{Y}$ est strictement négative, la partie Y n'est pas relativement compacte, d'après la proposition 6.1. On peut donc écrire

$$
\widehat{\mathrm{A}}_{\mathrm{Y}}=\bigcup_{n \geq 0} \widehat{\mathrm{A}}_{n}
$$

où $\left(\widehat{\mathrm{A}}_{n}\right)_{n \geq 0}$ est une famille strictement croissante de parties finies.

Fixons une arête $\alpha \in \widehat{\mathrm{A}}_{\mathrm{Y}}$ et choisissons une arête $\alpha^{\prime} \in \widehat{\pi}^{-1}(\alpha)$. L'arc frontière de

$$
\mathrm{X}_{-} \bigcup_{n \geq 0}(\varphi \cup \psi)^{n}\left(d\left(\alpha^{\prime}\right)\right)
$$

qui contient $\alpha^{\prime}$ est une droite de Brouwer $\Gamma_{\alpha^{\prime}}=\prod_{i=-\infty}^{+\infty} \alpha_{i}^{\prime}$, où $\alpha_{0}^{\prime}=\alpha^{\prime}$. Notons $\Gamma_{\alpha^{\prime}}^{+}$ la demi droite positive incluse dans $\Gamma_{\alpha^{\prime}}$ qui débute par $\alpha^{\prime}$. Posons $\alpha_{i}=\widehat{\pi}\left(\alpha_{i}^{\prime}\right)$ et définissons

$$
\widehat{\Gamma}_{\alpha}=\widehat{\pi}\left(\Gamma_{\alpha^{\prime}}\right)=\prod_{i=-\infty}^{+\infty} \alpha_{i}, \quad \widehat{\Gamma}_{\alpha}^{+}=\widehat{\pi}\left(\Gamma_{\alpha^{\prime}}^{+}\right)=\prod_{i=0}^{+\infty} \alpha_{i}
$$

Si l'arc $\widehat{\Gamma}_{\alpha}^{+}$est simple et contenu, à l'exception de sa source, dans $\mathrm{Y}$, il se relève en une famille de demi-droites de Brouwer $\mathrm{H}_{1}$-simples. Ces demi-droites sont même $\mathrm{H}_{0}$-simples. En effet une telle demi-droite ne peut rencontrer son image par $\mathrm{T} \in \mathrm{H}_{0} \backslash \mathrm{H}_{1}$ qu'en son origine, et celle-ci doit être fixe par $\mathrm{T}$, ce qui est impossible. Nous supposerons donc que pour tout arête $\alpha \in \widehat{\mathrm{A}}_{\mathrm{Y}}$, l'arc $\widehat{\Gamma}_{\alpha}^{+}$n'est pas simple ou rencontre $\partial \mathrm{Y}$ en un point différent de sa source. Il existe $i_{\alpha} \geq 0$ tel que l'arc $\prod_{i=0}^{i_{\alpha}} \alpha_{i}$ privé de ses extrémités est simple et contenu dans $\mathrm{Y}$ et tel que $b\left(\alpha_{i_{\alpha}}\right)$ appartient à $\partial \mathrm{Y}$ ou est égal à l'un des sommets $s\left(\alpha_{i}\right), 0 \leq i \leq i_{\alpha}$. On posera

$$
\widehat{\gamma}_{\alpha}=\prod_{i=0}^{i_{\alpha}} \alpha_{i}
$$


Pour tout $n \geq 0$, l'ensemble

$$
\mathrm{Z}_{n}=\mathrm{Z} \bigcup_{\alpha \in \widehat{\mathrm{A}}_{Y} \backslash \widehat{\mathrm{A}}_{n}} \widehat{\gamma}_{\alpha}
$$

est sans but et toutes les composantes connexes de son complémentaire incluses dans $\mathrm{Y}$ sont relativement compactes. D'après la proposition 6.1, il existe donc une arête $\alpha_{n} \notin \widehat{\mathrm{A}}_{n}$ telle que $\widehat{\gamma}_{\alpha_{n}}$ rencontre $\widehat{\gamma} \cup \widehat{\gamma}^{\prime}$. Écrivons

$$
\widehat{\gamma}_{\alpha_{n}}=\prod_{i=0}^{i_{n}} \alpha_{i}^{n} .
$$

Notons $\widehat{\mathrm{A}}_{*}$ l'ensemble des arêtes $\alpha \in \widehat{\mathrm{A}}_{\mathrm{Y}}$ qui rencontrent $\widehat{\gamma} \cup \widetilde{\gamma}^{\prime}$, et $\widehat{\mathrm{A}}_{* *}$ l'ensemble des arêtes qui rencontrent au moins une arête de $\widehat{\mathrm{A}}_{*}$. Pour tout $n \geq 0$, il existe $j_{n}<i_{n}$ tel $\alpha_{j_{n}}^{n} \in \widetilde{\mathrm{A}}_{* *}$. On peut extraire une suite convergente de la suite des arcs $\prod_{i=-\infty}^{j_{n}} \alpha_{i}^{n}$. C'est une demi-droite incluse dans Y puisque $\prod_{i=0}^{j_{n}} \alpha_{i}^{n}$ est simple et puisque $\lim _{n \rightarrow+\infty} j_{n}=+\infty$, elle se relève en une demi-droite de Brouwer qui est $\mathrm{H}_{1}$-simple et même $\mathrm{H}_{0}$-simple.

Cas iii). - On garde les mêmes notations que dans le cas précédent. Si $\mathrm{Y}$ est une partie compacte, on est nécessairement dans la situation où $\mathrm{X}_{-}=\emptyset$, $\mathrm{X}_{+}=\mathrm{B}$ et $\mathrm{H}$ isomorphe à $\mathbf{Z}^{2}$. C'est une situation étudiée en section 3. Sinon, $\mathrm{Y}$ est un anneau ouvert. Nous nous placerons dans ce cas. $\mathrm{Si} \mathrm{X}=\mathrm{K}_{0}$, nous nous retrouvons dans le cas i) puisque $\mathrm{H}_{0}=\mathrm{H}_{1}$ est abélien. On supposera donc $\mathrm{X} \neq \mathrm{K}_{0}$. L'ensemble $\partial \mathrm{X}_{+} \cap \partial \mathrm{X}_{-} \cap \mathrm{X}$ est alors non vide. Il est constitué de sommets dont la projection par $\widehat{\pi}$ sont des points de retournement extérieurs de Y, c'està-dire des sommets $\sigma$ qui sont but ou source d'une brique de $\mathrm{Y}$ dont les arêtes adjacentes d'extrémité $\sigma$ sont dans la frontière de Y. En d'autre termes on peut appliquer la proposition 6.3 : si $\mathrm{Y}$ était relativement compacte sa caractéristique d'Euler serait un, ce qui est impossible puisque $\mathrm{Y}$ est un anneau ouvert. Puisque Y n'est pas relativement compacte, on peut reprendre mot à mot la démonstration précédente (cas ii)) en considérant un lacet $\widehat{\gamma}$ définissant un élément non nul de $\pi_{1}(\mathrm{Y}, \widehat{z})$ et un segment $\widehat{\gamma}^{\prime}$ joignant $\widehat{z}$ à un sommet $\sigma \in \partial \mathrm{Y}$ correspondant à un retournement extérieur. Supposons qu'il soit de type source. On ne peut pas construire de partie sans but $Z^{\prime}$ en ajoutant à $Z$ des arêtes $\alpha \in \widehat{A}_{Y}$, qui ne rencontrent ni $\widehat{\gamma}$, ni $\widehat{\gamma}^{\prime}$ et telle que la composante connexe du complémentaire de $\mathrm{Z}^{\prime}$ contenant $\widehat{z}$ soit bornée. La caractéristique d'Euler de celle-ci devrait être égale à un, grâce à la proposition 6.2. On montre ainsi qu'il existe une demi-droite incluse dans $\mathrm{Y}$ qui se relève en une droite de Brouwer. Dans le cas où le point de retournement extérieur est de type but, on raisonne avec $\mathrm{F}^{-1}$ au lieu de $\mathrm{F}$. 
Il reste à démontrer que l'une au moins des trois propriétés est vérifiée. Il faut démontrer que si toutes les composantes non triviales de $\mathrm{X}_{+} \backslash \mathrm{X}_{-}$qui sont dans $\mathrm{K}_{0}$ ont un stabilisateur trivial dans $\mathrm{H}_{0}$ alors $\mathrm{H}_{0}$ est abélien. L'intérieur de toute composante est une composante connexe du complémentaire de l'ensemble sans but ni source $\partial X_{-} \cup \partial X_{+}$. Si une composante $X$ n'a pas de point de retournement sur sa frontière, alors $\mathrm{K}_{0}$ se réduit à cette composante. Puisqu'elle n'est pas triviale, $\mathrm{H}_{0}$ est donc trivial. On peut donc supposer que toute composante a $\mathrm{au}$ moins un point de retournement sur sa frontière. Une composante bornée en a exactement deux, un point source et un point but, d'après la proposition 6.2. Le même raisonnement que dans le cas iii) nous dit qu'une composante non bornée a également un but et une source et que l'intérieur est un disque ouvert. On peut choisir alors pour toute composante $\mathrm{X}$ un segment $\gamma_{\mathrm{X}}$ joignant les deux points de retournement de $\mathrm{X}$ et supposer que $\gamma_{\mathrm{TX}}=\mathrm{T} \gamma_{\mathrm{X}}$ pour tout $\mathrm{T} \in \mathrm{H}_{0}$. La réunion des arêtes $\alpha \in \mathrm{K}_{0} \cap \partial \mathrm{X}_{+} \cap \partial \mathrm{X}_{-}$et des segments $\gamma_{\mathrm{X}}$ est une variété topologique connexe de dimension un invariante sous l'action de $\mathrm{H}_{0}$. Ce groupe est donc trivial ou infini cyclique.

Pour démontrer la proposition 3.4, il reste à prouver :

Proposition 7.3. - Si $\mathscr{B}^{*}$ est un ensemble de droites de Brouwer n'ayant pas d'intersection transverse deux à deux, invariant sous l'action de $\mathrm{G}$, et maximal pour ces propriétés, la réunion des droites de $\mathscr{B}^{*}$ est égal au squelette $\Sigma(\mathscr{D})$ de la décomposition.

Démonstration. - On sait déjà que $\mathscr{B}^{*}$ est fermé dans $\mathscr{B}$ puisque $\mathscr{B}^{*}$ est maximal. On rappelle qu'on définit un ordre $\preceq$ sur $\mathscr{B}^{*}$ en posant :

$$
\Gamma \preceq \Gamma^{\prime} \text { si } \mathrm{D}(\Gamma) \subset \mathrm{D}\left(\Gamma^{\prime}\right) \text {. }
$$

On raisonne par l'absurde. On suppose qu'il existe une arête $\alpha_{0} \in$ A qui n'appartient à aucune droite de $\mathscr{B}^{*}$, et on va construire un ensemble de droites de Brouwer n'ayant pas d'intersection transverse deux à deux, invariant sous l'action de $\mathrm{G}$, et strictement plus grand que $\mathscr{B}^{*}$. On peut écrire $\mathscr{B}^{*}=\mathscr{B}_{+}^{*} \sqcup \mathscr{B}_{-}^{*}$, où

$$
\mathscr{B}_{+}^{*}=\left\{\Gamma \in \mathscr{B}^{*} \mid\left\{d\left(\alpha_{0}\right), g\left(\alpha_{0}\right)\right\} \subset \mathrm{D}(\Gamma)\right\}
$$

et

$$
\mathscr{B}_{-}^{*}=\left\{\Gamma \in \mathscr{B}^{*} \mid\left\{d\left(\alpha_{0}\right), g\left(\alpha_{0}\right)\right\} \subset \mathrm{G}(\Gamma)\right\} .
$$

Toute partie $\mathscr{B}^{\prime} \subset \mathscr{B}_{+}^{*}$ non vide totalement ordonnée admet un minorant dans $\mathscr{B}_{+}^{*}$. En effet, $\bigcup_{\Gamma \in \mathscr{B}^{\prime}} \mathrm{G}(\Gamma)$ est un répulseur connexe et toute composante connexe du complémentaire $\bigcap_{\Gamma \in \mathscr{B}} \mathrm{D}(\Gamma)$ est un attracteur ayant un unique arc frontière. 
Cette droite, qui appartient à $\mathscr{B}^{*}$ puisque $\mathscr{B}^{*}$ est fermé, est un minorant de $\mathscr{B}^{\prime}$. L'une des composantes contient $\left\{d\left(\alpha_{0}\right), g\left(\alpha_{0}\right)\right\}$ et sa droite frontière est dans $\mathscr{B}_{+}^{*}$. De même toute partie $\mathscr{B}^{\prime} \subset \mathscr{B}_{-}^{*}$ non vide totalement ordonnée admet un majorant dans $\mathscr{B}_{-}^{*}$. Notons $\mathscr{B}_{++}^{*}$ l'ensemble des éléments minimaux $\Gamma_{+}$de $\mathscr{B}_{+}^{*}$ tels qu'il n'existe aucune droite $\Gamma_{-} \in \mathscr{B}_{-}^{*}$ vérifiant $\mathrm{G}\left(\Gamma_{-}\right) \subset \mathrm{D}\left(\Gamma_{+}\right)$. De même, notons $\mathscr{B}_{--}^{*}$ l'ensemble des éléments maximaux $\Gamma_{-}$de $\mathscr{B}_{-}^{*}$ tels qu'il n'existe aucune droite $\Gamma_{+} \in \mathscr{B}_{+}^{*}$ vérifiant $\mathrm{D}\left(\Gamma_{+}\right) \subset \mathrm{G}\left(\Gamma_{-}\right)$. Remarquons que tout ensemble $\mathrm{D}(\Gamma)$, $\Gamma \in \mathscr{B}_{+}^{*}$, contient, soit un ensemble $\mathrm{D}(\Gamma), \Gamma \in \mathscr{B}_{++}^{*}$, soit un ensemble $\mathrm{G}(\Gamma)$, $\Gamma \in \mathscr{B}_{--}^{*}$. En effet, il existe un élément minimal $\Gamma_{+}$de $\mathscr{B}_{+}^{*}$ tel que $\mathrm{D}\left(\Gamma_{+}\right) \subset \mathrm{D}(\Gamma)$. Si $\Gamma_{+}$n'appartient pas à $\mathscr{B}_{++}^{*}$, il existe une droite $\Gamma_{-} \in \mathscr{B}_{-}^{*}$ telle que $\mathrm{G}\left(\Gamma_{-}\right) \subset \mathrm{D}\left(\Gamma_{+}\right)$et on peut la choisir maximale dans $\mathscr{B}_{-}^{*}$. Remarquons qu'elle est nécessairement dans $\mathscr{B}_{--}^{*}$ puisque, par minimalité de $\Gamma_{+}$, il n'existe pas de droite $\Gamma^{\prime} \in \mathscr{B}_{+}^{*}$ telle que $\mathrm{D}\left(\Gamma^{\prime}\right) \subset \mathrm{G}\left(\Gamma_{-}\right) \subset \mathrm{D}\left(\Gamma_{+}\right)$. De même, tout ensemble $\mathrm{G}(\Gamma), \Gamma \in \mathscr{B}_{-}^{*}$, contient, soit un ensemble $\mathrm{D}(\Gamma), \Gamma \in \mathscr{B}_{++}^{*}$, soit un ensemble $G(\Gamma), \Gamma \in \mathscr{B}_{--}^{*}$. On définit ensuite les attracteurs

$$
\mathrm{X}_{+}=\bigcap_{\Gamma \in \mathscr{B}_{++}^{*}} \mathrm{D}(\Gamma), \quad \mathrm{X}_{-}=\bigcup_{\Gamma \in \mathscr{B}_{--}^{*}} \mathrm{D}(\Gamma) .
$$

Remarquons, par définition de $\mathscr{B}_{++}^{*}$ et $\mathscr{B}_{--}^{*}$, que pour toutes droites $\Gamma_{-} \in$ $\mathscr{B}_{--}^{*}, \Gamma_{+} \in \mathscr{B}_{++}^{*}$, on a

$$
\mathrm{G}\left(\Gamma_{-}\right) \not \subset \mathrm{D}\left(\Gamma_{+}\right), \quad \mathrm{D}\left(\Gamma_{+}\right) \not \subset \mathrm{G}\left(\Gamma_{-}\right)
$$

et donc

$$
\mathrm{D}\left(\Gamma_{-}\right) \subset \mathrm{D}\left(\Gamma_{+}\right)
$$

En d'autres termes, on a $\mathrm{X}_{-} \subset \mathrm{X}_{+}$.

On note $\mathrm{H}$ le stabilisateur (commun) de $\mathscr{B}_{+}^{*}$ et $\mathscr{B}_{-}^{*}$. Il laisse invariant $\mathscr{B}_{++}^{*}$, $\mathscr{B}_{--}^{*}, \mathrm{X}_{+}$et $\mathrm{X}_{-}$. Puisque

$$
d\left(\alpha_{0}\right) \cup g\left(\alpha_{0}\right) \subset \mathrm{X}_{+} \backslash \operatorname{Int}\left(\mathrm{X}_{-}\right),
$$

on peut appliquer le lemme 7.2. Il existe une droite de Brouwer $\Gamma \subset \mathrm{X}_{+} \backslash \operatorname{Int}\left(\mathrm{X}_{-}\right)$ qui est H-presque simple et qui contient une arête $\alpha$ telle que

$$
d(\alpha) \cup g(\alpha) \subset \mathrm{X}_{+} \backslash \operatorname{Int}\left(\mathrm{X}_{-}\right) .
$$

Gette arête n'appartient à aucune droite $\Gamma^{\prime} \in \mathscr{B}^{*}$. En effet, $\{d(\alpha), g(\alpha)\}$ est inclus dans toute partie $\mathrm{D}\left(\Gamma^{\prime}\right), \Gamma^{\prime} \in \mathscr{B}_{++}^{*}$ et dans toute partie $\mathrm{G}\left(\Gamma^{\prime}\right), \Gamma^{\prime} \in \mathscr{B}_{--}^{*}$, elle est donc contenue dans tout $\mathrm{D}\left(\Gamma^{\prime}\right), \Gamma^{\prime} \in \mathscr{B}_{+}^{*}$ et dans tout $\mathrm{G}\left(\Gamma^{\prime}\right), \Gamma^{\prime} \in \mathscr{B}_{-}^{*}$, par définition de $\mathscr{B}_{++}^{*}$ et $\mathscr{B}_{--}^{*}$. Pour les mêmes raisons, $\Gamma$ est incluse dans tout $\mathrm{D}\left(\Gamma^{\prime}\right)$, 
$\Gamma^{\prime} \in \mathscr{B}_{+}^{*}$ et dans tout $\mathrm{G}\left(\Gamma^{\prime}\right), \Gamma^{\prime} \in \mathscr{B}_{-}^{*}$, ce qui prouve que $\Gamma$ n'a pas d'intersection transverse avec une droite $\Gamma^{\prime} \in \mathscr{B}^{*}$.

Montrons maintenant que $\Gamma$ n'a pas d'intersection transverse avec TГ si $\mathrm{T} \notin \mathrm{H}$. Puisque $\mathrm{T}$ ne stabilise pas $\mathscr{B}_{+}^{*}$, on peut supposer, quitte à changer $\mathrm{T}$ en $\mathrm{T}^{-1}$, qu'il existe une droite $\Gamma_{-} \in \mathscr{B}_{-}^{*}$ telle que $\mathrm{T}^{-1} \Gamma_{-} \in \mathscr{B}_{+}^{*}$. On a donc

$$
\Gamma \subset \mathrm{G}\left(\Gamma_{-}\right) \cap \mathrm{D}\left(\mathrm{T}^{-1} \Gamma_{-}\right)
$$

autrement dit

$$
\Gamma \subset \mathrm{G}\left(\Gamma_{-}\right), \quad \mathrm{T}(\Gamma) \subset \mathrm{D}\left(\Gamma_{-}\right),
$$

ainsi les droites $\Gamma$ et $\mathrm{T}(\Gamma)$ n'ont pas d'intersection transverse. L'ensemble formé des droites $\Gamma^{\prime} \in \mathscr{B}^{*}$ et des droites $\mathrm{T} \Gamma, \mathrm{T} \in \mathrm{G}$, est strictement plus grand que $\mathscr{B}^{*}$.

\section{Applications du théorème d'existence de feuilletage trans- verse}

\section{Feuilletages transverses et vecteurs de rotation}

Commençons par rappeler la notion de vecteur de rotation, dont l'idée remonte à Schwartzman [Scn]. Nous nous limiterons ici aux vecteurs de rotation des points récurrents. Soit $\mathrm{M}$ une variété (éventuellement à bord), et $\left(\mathrm{F}_{t}\right)_{t \in[0,1]}$ une isotopie issue de l'identité et aboutissant à un homéomorphisme $\mathrm{F}$. Pour tout $z \in \mathrm{M}$, on note $\gamma_{z}$ l'arc $t \mapsto \mathrm{F}_{t}(z)$. Rappelons que $[\Gamma] \in \mathrm{H}_{1}(\mathbf{M}, \mathbf{Z})$ est la classe d'homologie d'un lacet $\Gamma$ dans M. Soit $z_{0}$ un point positivement récurrent de F. Considérons une boule ouverte $\mathrm{U}$ contenant $z_{0}$ et choisissons, pour tout couple $\left(z, z^{\prime}\right)$ de points de $\mathrm{U}$ un arc $\gamma_{z, z^{\prime}}$ à valeurs dans $\mathrm{U}$ joignant $z$ à $z^{\prime}$. Pour tout entier $n \geq 1$ tel que $\mathrm{F}^{n}\left(z_{0}\right) \in \mathrm{U}$, on peut définir le lacet

$$
\Gamma_{n}=\left(\prod_{i=0}^{n-1} \gamma_{\mathrm{F}^{i}\left(z_{0}\right)}\right) \gamma_{\mathrm{F}^{n}\left(z_{0}\right), z_{0}} .
$$

On dira que le point $z_{0}$ a un vecteur de rotation $\rho\left(z_{0}\right) \in \mathrm{H}_{1}(\mathbf{M}, \mathbf{R})$ si, pour toute suite $\left(n_{k}\right)_{k \geq 0}$ d'entiers positifs :

$$
\lim _{k \rightarrow+\infty} \mathrm{F}^{n_{k}}\left(z_{0}\right)=z_{0} \Longrightarrow \lim _{k \rightarrow+\infty} \frac{1}{n_{k}}\left[\Gamma_{n_{k}}\right]=\rho\left(z_{0}\right) .
$$

G'est le cas par exemple pour un point périodique, le vecteur de rotation appartient alors à $H_{1}(M, \mathbf{Q})$. Il est clair que l'existence et la valeur du vecteur de 
rotation d'un point récurrent $z_{0}$ sont indépendantes de la boule $U$ ainsi que de la famille $\left(\gamma_{z, z^{\prime}}\right)_{z, z^{\prime}}$ choisies. Il est clair également que le vecteur de rotation est bien défini et constant sur l'orbite de $z$. Si $\mathrm{R} \subset \mathrm{M}$ est l'ensemble des points positivement récurrents de $\mathrm{F}$, on peut définir l'application de premier retour

$$
\begin{aligned}
\Phi: \mathrm{R} \cap \mathrm{U} & \rightarrow \mathrm{R} \cap \mathrm{U} \\
z & \mapsto \Phi(z)=\mathrm{F}^{\tau(z)}(z),
\end{aligned}
$$

où $\tau(z)$ est le temps de premier retour, et pour tout $z \in \mathrm{R} \cap \mathrm{U}$ le lacet

$$
\Gamma_{z}=\left(\prod_{i=0}^{\tau(z)-1} \gamma_{\mathrm{F}^{i}(z)}\right) \gamma_{\Phi(z), z} .
$$

Si, pour $n \geq 1$, on pose

$$
\tau_{n}(z)=\sum_{i=0}^{n-1} \tau\left(\Phi^{i}(z)\right)
$$

on remarque que

$$
\left[\left(\prod_{j=0}^{\tau_{n}(z)-1} \gamma_{\mathbf{F}^{j}(z)}\right) \gamma_{\Phi^{n}(z), z}\right]=\sum_{i=0}^{n-1}\left[\Gamma_{\Phi^{i}(z)}\right] .
$$

On en déduit que si la suite

$$
\left(\frac{1}{\tau_{n}(z)} \sum_{i=0}^{n-1}\left[\Gamma_{\Phi^{i}(z)}\right]\right)_{n \geq 1}
$$

est convergente, alors $z$ a un vecteur de rotation et

$$
\rho(z)=\lim _{n \rightarrow+\infty} \frac{1}{\tau_{n}(z)} \sum_{i=0}^{n-1}\left[\Gamma_{\Phi^{i}(z)}\right] .
$$

Si $\mu$ est une mesure borélienne de probabilité invariante par $\mathrm{F}$, nous savons que presque tout point est positivement récurrent, que l'application $\tau$ est intégrable (c'est le classique lemme de Kac) et que

$$
\int_{\mathrm{U}} \tau d \mu=\mu\left(\bigcup_{k \in \mathbf{N}} \mathrm{F}^{k}(\mathrm{U})\right)=\mu\left(\bigcup_{k \in \mathbf{Z}} \mathrm{F}^{k}(\mathrm{U})\right) .
$$


Grâce au théorème ergodique de Birkhoff, nous en déduisons que la suite $\left(\frac{\tau_{n}(z)}{n}\right)_{n \geq 1}$ converge presque surement sur $\mathrm{R} \cap \mathrm{U}$. Si de plus $\mathrm{M}$ est une variété compacte, la fonction $z \mapsto \frac{\left[\Gamma_{z}\right]}{\tau(z)}$ est bornée sur $\mathrm{R} \cap \mathrm{U}$ et la fonction $z \mapsto\left[\Gamma_{z}\right]$ est donc intégrable. Utilisant encore une fois le théorème de Birkhoff nous en déduisons que pour presque tout point $z \in \mathrm{R} \cap \mathrm{U}$, la suite

$$
\left(\frac{1}{\tau_{n}(z)} \sum_{i=0}^{n-1}\left[\Gamma_{\Phi^{i}(z)}\right]\right)_{n \geq 1}
$$

converge dans $\mathrm{H}_{1}(\mathrm{M}, \mathrm{R})$, en particulier presque tout point $z \in \mathrm{R}$ a un vecteur de rotation. La fonction $\rho: \mathrm{M} \rightarrow \mathrm{H}_{1}(\mathrm{M}, \mathbf{R})$ ainsi définie est bornée et on définit le vecteur de rotation de la mesure

$$
\rho(\mu)=\int_{\mathrm{M}} \rho d \mu \in \mathrm{H}_{1}(\mathrm{M}, \mathbf{R}) \text {. }
$$

Étudions maintenant le cas particulier où $\mathrm{M}$ est une surface (orientable). Pour parler de vecteur de rotation, on doit exclure le cas où $M$ est un disque ou la sphère. Dans le cas où $M$ est le tore de dimension deux ou un anneau, la valeur du vecteur de rotation d'un point est déterminée par la donnée d'un relèvement $f$ de $\mathrm{F}$ au revêtement universel de $\mathrm{M}$. Dans les autres cas, c'est-à-dire quand $\mathrm{M}$ est de type infini, ou alors de type fini et de caractéristique d'Euler négative, la classe d'homotopie de chaque lacet $\gamma_{z}$ est indépendante de l'isotopie $\left(\mathrm{F}_{t}\right)_{t \in[0,1]}$. Ainsi le vecteur de rotation d'un point ou d'une mesure ne dépend pas de l'isotopie choisie, il est déterminé par F. Dans ces derniers cas, un homéomorphisme admet au plus un relèvement au revêtement universel qui commute avec tous les automorphismes du revêtement (la raison principale est que le centre du groupe fondamental est trivial). En fait sur une surface compacte de genre $\geq 2$, deux isotopies joignant l'identité à un homéomorphisme $\mathrm{F}$ sont toujours homotopes (voir M.-E. Hamstrom [Ham1], [Ham2]). Dans le cas où $\mathbf{M}$ est un anneau, on peut parler de vecteurs de rotation de mêmes signes ou de signes opposés, on peut également définir le nombre de rotation $v(z) \in \mathbf{R}$ d'un point récurrent $z$, dès qu'on se donne un lacet simple $\Gamma^{*}$ non homotope à zéro, en posant $\rho(z)=v(z)\left[\Gamma^{*}\right]$. Dans ce contexte, on peut parler d'un point récurrent qui a un nombre de rotation égal à $+\infty$ ou à $-\infty$. Remarquons, dans le cas où $M$ est un anneau compact, que l'on peut parler du vecteur de rotation induit sur chaque bord.

Rappelons l'énoncé du théorème 1.3 démontré dans la partie I :

Théorème 8.1. - Soit $\mathrm{M}$ une surface (sans bord) et $\left(\mathrm{F}_{t}\right)_{t \in[0,1]}$ une isotopie dans $\mathrm{M}$ joignant l'identité à un homéomorphisme F. On suppose que $\mathrm{F}$ n'a pas de point fixe contractile. Il existe alors un feuilletage topologique orienté $\mathscr{F}$ sur $\mathrm{M}$ et pour tout $z \in \mathrm{M}$, un arc positivement transverse à $\mathscr{F}$ joignant $z$ à $\mathrm{F}(z)$ qui est homotope, à extrémités fixées, à l'arc $\gamma_{z}: t \mapsto \mathrm{F}_{t}(z)$. 
On rappelle que le feuilletage $\mathscr{F}$ et l'isotopie $\left(\mathrm{F}_{t}\right)_{t \in[0,1]}$ sont dits transverses. Un cas important d'application du théorème est le suivant. Soit $\mathrm{M}$ une surface et $\left(\mathrm{F}_{t}\right)_{t \in[0,1]}$ une isotopie dans $\mathrm{M}$ joignant l'identité à $\mathrm{F}$. On suppose que l'ensemble $\operatorname{Fix}_{*}(\mathrm{~F})$ des points fixes contractiles est fini. On dira qu'une partie $\mathrm{X} \subset \operatorname{Fix}_{*}(\mathrm{~F})$ est non enlacée s'il existe une isotopie $\left(\mathrm{F}_{t}^{\prime}\right)_{t \in[0,1]}$ de l'identité à $\mathrm{F}$, fixant tout point de $\mathrm{X}$ et telle que les arcs $t \mapsto \mathrm{F}_{t}^{\prime}(z)$ et $t \mapsto \mathrm{F}_{t}(z)$ sont homotopes dans M. Si, de plus, $\mathrm{X}$ est maximale au sens de l'inclusion dans l'ensemble des parties non enlacées, on dira que $\mathrm{X}$ est une partie non enlacée maximale. Dans ce cas, si $\left(\widehat{\mathrm{F}}_{t}^{\prime}\right)_{t \in[0,1]}$ est l'isotopie relevée au revêtement universel de $\mathrm{M} \backslash \mathrm{X}$ issue de l'identité, alors $\widehat{\mathrm{F}}_{1}^{\prime}$ n'a pas de point fixe. On peut alors appliquer le théorème à l'isotopie $\left(\mathrm{F}_{t}^{\prime}\right)_{t \in[0,1]}$ restreinte à $\mathrm{M} \backslash \mathrm{X}$, le feuilletage transverse $\mathscr{F}$ obtenu admet $\mathrm{X}$ comme ensemble de singularités. En effet si $\widehat{\mathrm{F}}_{1}^{\prime}$ avait un point fixe, ce point se projetterait sur un point fixe contractile $z_{0}$ de $\left.\mathrm{F}\right|_{\mathrm{M} \backslash \mathrm{X}}$. Il est alors possible de trouver une isotopie $\left(\mathrm{F}_{t}^{\prime \prime}\right)_{t \in[0,1]}$ sur $\mathrm{M}$, fixant tout point de $\mathrm{X} \cup\left\{z_{0}\right\}$ et telle que chaque arc $t \mapsto \mathrm{F}_{t}^{\prime \prime}(z), z \notin \mathrm{X}$, est homotope à $t \mapsto \mathrm{F}_{t}^{\prime}(z)$ dans $\mathrm{M} \backslash \mathrm{X}$ et donc homotope à $t \mapsto \mathrm{F}_{t}(z)$ dans $\mathrm{M}$, ce qui contredit la maximalité de $\mathrm{X}$ parmi les parties non enlacées. Soyons plus précis et pour cela considérons une application continue

$$
\Psi:[0,1] \times[0,1] \rightarrow \mathrm{M} \backslash \mathrm{X}
$$

telle que, pour tout $t \in[0,1]$, on a :

$$
\begin{aligned}
& \Psi(0, t)=\mathrm{F}_{t}^{\prime}\left(z_{0}\right), \\
& \Psi(1, t)=z_{0} .
\end{aligned}
$$

Munissons $\mathrm{M}$ d'une structure riemannienne et notons $d$ la distance sur $\mathrm{M}$ induite par cette structure. L'ensemble $\Psi\left([0,1]^{2}\right)$ étant compact et disjoint de $\mathrm{X}$, on peut trouver $\delta>0$ tel que, pour tout $z \in \Psi\left([0,1]^{2}\right)$, l'application exponentielle induit un difféomorphisme entre les ensembles $\left\{u \in \mathrm{T}_{z} \mathrm{M} \mid\|u\|<\delta\right\}$ et $\left\{z^{\prime} \in \mathrm{M} \mid d\left(z, z^{\prime}\right)<\delta\right\}$. On peut de plus supposer que $d\left(z, z^{\prime}\right) \geq \delta$ pour tout $\left(z, z^{\prime}\right) \in \Psi\left([0,1]^{2}\right) \times \mathrm{X}$. On peut trouver un entier $\mathrm{N} \geq 1$ tel que pour tout $s$, $s^{\prime}$ et $t$ dans $[0,1]$, on a :

$$
\left|s-s^{\prime}\right| \leq \frac{1}{\mathrm{~N}} \Longrightarrow d\left(\Psi(s, t), \Psi\left(s^{\prime}, t\right)\right) \leq \frac{\delta}{3} \text {. }
$$

Posons

$$
\mathrm{K}=\left\{\left(z, z^{\prime}\right) \in \mathrm{M} \mid z \in \mathrm{K}, d\left(z, z^{\prime}\right) \leq \frac{\delta}{3}\right\} .
$$

Il n'est pas difficile de construire, pour tout $\left(z, z^{\prime}\right) \in \mathrm{K}$, un champ de vecteurs $\xi_{z, z^{\prime}}$ de classe $\mathrm{C}^{1}$, dépendant continûment de $\left(z, z^{\prime}\right)$, tel que $\xi_{z, z^{\prime}}\left(z^{\prime \prime}\right)=0$ si $d\left(z, z^{\prime \prime}\right) \geq$ $2 d\left(z, z^{\prime}\right)$ et tel que $\Phi_{z, z^{\prime}}^{\frac{1}{N}}\left(z^{\prime}\right)=z$, où $\left(\Phi_{z, z^{\prime}}^{t}\right)_{t \in \mathbf{R}}$ est le flot induit par $\xi_{z, z^{\prime}}$. 
Pour tout $(s, t) \in[0,1]$ posons :

$$
\mathrm{F}_{t}^{s}=\Phi_{\Psi\left(\frac{[N s]+1}{N}, t\right), \Psi\left(\frac{[N s]}{N}, t\right)}^{s-\frac{[N s]}{N}} \circ \Phi_{\Psi\left(\frac{1 N s]}{N}, t\right), \Psi\left(\frac{[N s]-1}{N}, t\right)}^{\frac{1}{N}} \circ \ldots \circ \Phi_{\Psi\left(\frac{1}{N}, t\right), \Psi(0, t)}^{\frac{1}{N}} \circ \mathrm{F}_{t}^{\prime} .
$$

Remarquons que la famille d'homéomorphismes $\left(\mathrm{F}_{t}^{s}\right)_{(s, t) \in[0,1]^{2}}$ dépend continûment de $(s, t)$, que chaque $\mathrm{F}_{t}^{s}$ laisse fixe tous les points de $\mathrm{X}$, que chaque $\mathrm{F}_{t}^{1}$ laisse fixe $z_{0}$ et que $\mathrm{F}_{t}^{0}=\mathrm{F}_{t}^{\prime}$. L'ensemble $\mathrm{X} \cup\left\{z_{0}\right\}$ est non enlacé. de $\mathscr{F}$ :

Nous allons conclure cette section en reliant la dynamique de $\mathrm{F}$ à celle

Proposition 8.2. - Soit $\mathrm{M}$ une surface et $\left(\mathrm{F}_{t}\right)_{t \in[0,1]}$ une isotopie dans $\mathrm{M}$ joignant l'identité à un homéomorphisme $\mathrm{F}$. On suppose que $\mathrm{F}$ n'a pas de point fixe contractile et que $\mathscr{F}$ est un feuilletage transverse à l'isotopie. Nous avons alors les résultats suivants :

i) Pour tout point non errant $z$ de $\mathrm{F}$, il existe un lacet $\Gamma$ basé en $z$ qui est positivement transverse à $\mathscr{F}$.

ii) Pour tout point positivement récurrent $z$ de $\mathrm{F}$ qui admet un vecteur de rotation $\rho(z)$, il existe une suite $\left(\Gamma_{k}\right)_{k \geq 0}$ de lacets positivement transverses à $\mathscr{F}$ basés en $z$ et une suite d'entiers strictement positifs $\left(n_{k}\right)_{k \geq 0}$ telles que $\lim _{k \rightarrow \infty} \frac{1}{n_{k}}\left[\Gamma_{k}\right]=\rho(z)$.

iii) Si F n'a pas de point errant, pour tout couple de points $\left(z, z^{\prime}\right)$ de $\mathrm{M}$, il existe un arc positivement transverse à $\mathscr{F}$ qui joint z à $z^{\prime}$.

iv) Supposons que F n'ait pas de point errant et fixons $z_{0} \in \mathrm{M}$. Pour tout point positivement récurrent $z$ de $\mathrm{F}$ qui admet un vecteur de rotation $\rho(z)$, il existe une suite $\left(\Gamma_{k}^{0}\right)_{k \geq 0}$ de lacets positivement transverses à $\mathscr{F}$ basés en $z_{0}$ et une suite d'entiers strictement positifs $\left(n_{k}\right)_{k \geq 0}$ telles que $\lim _{k \rightarrow \infty} \frac{1}{n_{k}}\left[\Gamma_{k}^{0}\right]=\rho(z)$.

Démonstration. - Pour tout point $z \in \mathrm{M}$, il existe un arc positivement transverse à $\mathscr{F}$ qui joint $z$ à $\mathrm{F}(z)$ et qui est homotope à $\gamma_{z}: t \mapsto \mathrm{F}_{t}(z)$. On en déduit qu'il existe un disque ouvert $\mathrm{U}_{z}$ contenant $z$ et un disque ouvert $\mathrm{V}_{\mathrm{F}(z)}$ contenant $\mathrm{F}(z)$ tel que tout point $z^{\prime} \in \mathrm{U}_{z}$ peut être joint à tout point $z^{\prime \prime} \in \mathrm{V}_{\mathrm{F}(z)}$ par un arc positivement transverse à $\mathscr{F}$ et homotope à un arc $\gamma_{z^{\prime}, z} \gamma_{z} \gamma_{\mathrm{F}(z), z^{\prime \prime}}^{\prime}$, où $\gamma_{z^{\prime}, z}$ joint $z^{\prime}$ à $z$ dans $\mathrm{U}_{z}$ et $\gamma_{\mathrm{F}(z), z^{\prime \prime}}^{\prime}$ joint $\mathrm{F}(z)$ à $z^{\prime \prime}$ dans $\mathrm{V}_{\mathrm{F}(z)}$.

Supposons que $z$ soit non errant. On peut trouver un point $z^{\prime} \in \mathrm{V}_{\mathrm{F}(z)}$ et un entier $n \geq 0$ tel que $\mathrm{F}^{n}\left(z^{\prime}\right) \in \mathrm{U}_{\mathrm{F}^{-1}(z)}$. On peut construire une suite $\left(\gamma_{i}\right)_{0 \leq i \leq n+1}$ d'arcs positivement transverses à $\mathscr{F}$, où :

$-\gamma_{0}$ joint $z$ à $z^{\prime}$;

$-\gamma_{i}$ joint $\mathrm{F}^{i-1}\left(z^{\prime}\right)$ à $\mathrm{F}^{i}\left(z^{\prime}\right)$ si $1 \leq i \leq n$;

$-\gamma_{n+1}$ joint $\mathrm{F}^{n}\left(z^{\prime}\right)$ à $z$.

On obtient l'assertion i) en considérant le lacet $\prod_{i=0}^{n+1} \gamma_{i}$. 
Supposons que $z$ soit positivement récurrent et ait un vecteur de rotation $\rho(z)$. Considérons une suite $\left(n_{k}\right)_{k \geq 0}$ telle que $\lim _{k \rightarrow+\infty} \mathrm{F}^{n_{k}}(z)=z$. Pour tout $i \geq 0$ on considère un arc $\gamma_{i}$ positivement transverse à $\mathscr{F}$ joignant $\mathrm{F}^{i}(z)$ à $\mathrm{F}^{i+1}(z)$ et homotope à $\gamma_{\mathrm{F}^{i}(z)}$. Pour $k$ assez grand, le point $\mathrm{F}^{n_{k}-1}(z)$ appartient à $\mathrm{U}_{\mathrm{F}^{-1}(z)}$ et on peut trouver un arc $\gamma^{k}$ positivement transverse à $\mathscr{F}$ qui joint $\mathrm{F}^{n_{k}-1}(z)$ à $z$ et

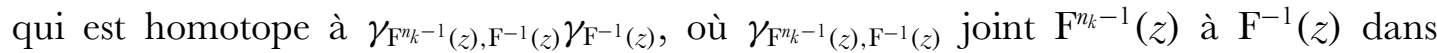
$\mathrm{U}_{\mathrm{F}^{-1}(z)}$. Le lacet $\Gamma_{k}=\left(\prod_{i=0}^{n_{k}-2} \gamma_{i}\right) \gamma^{k}$ est transverse à $\mathscr{F}$ et on a

$$
\rho(z)=\lim _{k \rightarrow+\infty} \frac{1}{n_{k}}\left[\Gamma_{k}\right] .
$$

Pour montrer l'assertion iii) fixons $z_{0} \in \mathrm{M}$ et considérons l'ensemble $\mathrm{N}$ des points $z \in \mathrm{M}$ tels qu'il existe un arc positivement transverse à $\mathscr{F}$ joignant $z_{0}$ à $z$. Cet ensemble est positivement invariant par F. On a mieux. En effet, pour tout point $z \in \mathrm{M}$, on a $\mathrm{V}_{\mathrm{F}(z)} \subset \mathrm{N}$ dès que $\mathrm{U}_{z} \cap \mathrm{N} \neq \emptyset$, en particulier on a $\mathrm{F}(\overline{\mathrm{N}}) \subset$ $\operatorname{Int}(\mathrm{N})$. Si l'inclusion précédente était stricte, $\operatorname{Int}(\mathrm{N}) \backslash \mathrm{F}(\overline{\mathrm{N}})$ serait une partie ouverte errante. On a donc $\mathrm{F}(\overline{\mathrm{N}})=\operatorname{Int}(\mathrm{N})$ et on en déduit que $\mathrm{N}=\mathrm{M}$ puisque $\mathrm{M}$ est connexe et que $\operatorname{Int}(\mathrm{N})$ est ouvert et fermé dans $\mathrm{M}$.

La dernière proposition se déduit immédiatement des deux précédentes. On sait qu'il existe un arc $\gamma$ positivement transverse à $\mathscr{F}$ joignant $z_{0}$ à $z$ et un arc $\gamma^{\prime}$ positivement transverse à $\mathscr{F}$ joignant $z$ à $z_{0}$. En gardant les notations introduites pour prouver la condition iii), on obtient

$$
\rho(z)=\lim _{k \rightarrow+\infty} \frac{1}{n_{k}}\left[\Gamma_{k}^{0}\right],
$$

si on pose $\Gamma_{k}^{0}=\gamma \Gamma_{k} \gamma^{\prime}$.

\section{Premières applications de l'existence d'un feuilletage transverse}

Considérons un homéomorphisme $\mathrm{F}$ de l'anneau $\mathbf{T}^{1} \times[0,1]$ préservant l'aire et supposons que les vecteurs de rotation induits sur les bords (pour un relèvement donné $f$ de $\mathrm{F}$ à $\mathbf{R} \times[0,1])$ soient non nuls et de signes opposés. Le théorème de Poincaré-Birkhoff nous dit que $\mathrm{F}$ admet au moins deux points fixes de vecteurs de rotation zéro. On sait depuis longtemps (voir Birkhoff [Bi], Kerékjártó [K] ou encore Guillou [Gu] pour une explication plus moderne) que l'existence d'un point fixe peut se déduire de la propriété d'intersection suivante, plus faible que la préservation de l'aire :

tout lacet simple non homotope à zéro rencontre son image par F.

Si tout lacet simple non homotope à zéro rencontre son image en au moins deux points (ce qui est le cas si $\mathrm{F}$ préserve l'aire), on sait d'après P. Carter [Ca] 
qu'il y a au moins deux points fixes. La proposition qui suit, due à Franks, affaiblit les hypothèses pour l'obtention d'un point fixe :

Théorème 9.1. - Soit $\mathrm{F}$ un homéomorphisme isotope à l'identité d'un anneau ouvert $\mathrm{A}$ et $f$ un relèvement de $\mathrm{F}$ au revêtement universel de $\mathrm{A}$. On suppose que :

- $\mathrm{F}$ admet deux points positivement récurrents $z_{0}$ et $z_{1}$ de vecteurs de rotation non nuls (pour $f$ ) et de signes contraires ;

- F n'admet pas de point fixe de vecteur de rotation nul.

Il existe alors un lacet simple non homotope à zéro disjoint de son image par $\mathrm{F}$ et qui sépare $z_{0}$ et $z_{1}$.

Démonstration. - Considérons une isotopie $\left(\mathrm{F}_{t}\right)_{t \in[0,1]}$ se relevant en une isotopie $\left(f_{t}\right)_{t \in[0,1]}$ de l'identité à $f$. Nous savons, par hypothèse, qu'il n'y a pas de point fixe contractile et nous pouvons donc construire un feuilletage $\mathscr{F}$ transverse à l'isotopie. Grâce à la proposition 8.2 ii), on peut trouver deux lacets $\Gamma_{0}$ et $\Gamma_{1}$ positivement transverses à $\mathscr{F}$ et deux entiers $n_{0}>0$ et $n_{1}>0$ tels que

$$
\left[\Gamma_{0}\right] \neq 0, \quad\left[\Gamma_{1}\right] \neq 0, \quad n_{0}\left[\Gamma_{0}\right]+n_{1}\left[\Gamma_{1}\right]=0 .
$$

Il existe alors une fonction $\Lambda: \mathrm{A} \backslash\left(\Gamma_{0} \cup \Gamma_{1}\right) \rightarrow \mathbf{Z}$, localement constante, uniquement définie par les propriétés suivantes :

$-\Lambda(z)=0$ si $z$ appartient à une composante connexe non relativement compacte de $\mathrm{A} \backslash\left(\Gamma_{0} \cup \Gamma_{1}\right)$;

$-\Lambda\left(z^{\prime}\right)-\Lambda(z)$ représente le nombre d'intersection algébrique

$$
n_{0} \Gamma \wedge \Gamma_{0}+n_{1} \Gamma \wedge \Gamma_{1}
$$

où $\Gamma$ est un arc quelconque joignant $z$ à $z^{\prime}$.

Quitte à perturber $\Gamma_{0}$ et $\Gamma_{1}$, on peut supposer que ces arcs vérifient les propriétés suivantes :

- $\Gamma_{0}$ et $\Gamma_{1}$ n'ont pas de point triple ou d'ordre supérieur ;

- $\Gamma_{0}$ et $\Gamma_{1}$ ont un nombre fini de points doubles et les auto-intersections sont transverses ;

- $\Gamma_{0}$ et $\Gamma_{1}$ ont un nombre fini de points d'intersection et les intersections sont transverses.

La fonction $\Lambda$ n'est pas constante et on doit avoir $\max \Lambda>0$ ou $\min \Lambda<0$. Plaçons nous dans le premier cas et considérons une composante connexe $\mathrm{U}$ de $\mathrm{M} \backslash\left(\Gamma_{0} \cup \Gamma_{1}\right)$ où $\Lambda$ atteint son maximum. Remarquons d'abord que la frontière de $\mathrm{U}$ est une variété topologique de dimension 1. Il suffit pour cela de regarder 
quelles valeurs prend la fonction $\Lambda$ au voisinage d'un point d'auto-intersection de la "multi-courbe" $n_{0} \Gamma_{0}+n_{1} \Gamma_{1}$ qui est sur la frontière de $\mathrm{U}$.

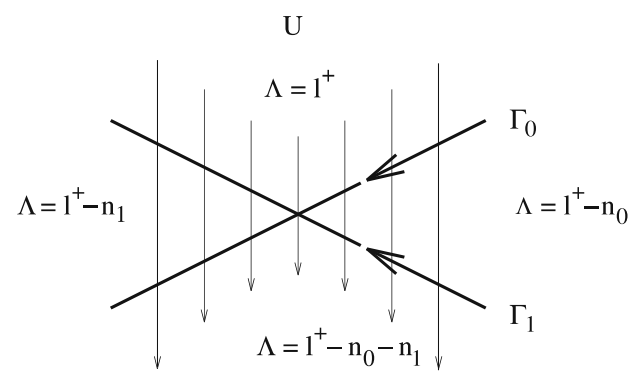

Remarquons également que la multi-courbe induit sur le bord de U une orientation et que $\mathrm{U}$ est à droite de son bord pour cette orientation. Finalement la multi-courbe étant positivement transverse au feuilletage, ce feuilletage est transverse au bord de $\mathrm{U}$ et sortant. Puisque le feuilletage est sans singularité et transverse au bord, la formule de Poincaré-Hopf nous dit que U est un anneau et que sa frontière a deux composantes connexes. De plus, l'anneau est essentiel puisque le feuilletage est non singulier. Le thèorème de Poincaré-Bendixson nous dit alors qu'il existe une feuille fermée $\phi$ dans U. Cette feuille est disjointe de son image par F, puisqu'il est impossible de trouver un arc transverse à $\mathscr{F}$ joignant deux points de $\phi$. Plus précisément l'image de $\phi$ est contenue dans la composante connexe de $\mathrm{A} \backslash \phi$ située "à droite" de $\phi$. Remarquons maintenant que $\phi$ qui est disjointe de la multi-courbe sépare nécessairement les deux courbes $\Gamma_{0}$ et $\Gamma_{1}$ et donc en particulier $z_{0}$ et $z_{1}$.

En appliquant le théorème précédent aux itérés $\mathrm{F}^{q}$ et aux relèvements $f^{q} \circ \mathrm{T}^{-p}$ de $\mathrm{F}^{q}$, on obtient le résultat suivant :

Corollaire 9.2. - Soit $\mathrm{F}$ un homéomorphisme isotope à l'identité d'un anneau ouvert A, vérifiant la propriété d'intersection, et $f$ un relèvement au revêtement universel de A. Fixons un lacet simple de $\mathrm{A}$ non homotope à zéro pour défnir les nombres de rotation. On suppose que $\mathrm{F}$ admet un point positivement récurrent de nombre de rotation $v^{-}$et un point positivement récurrent de nombre de rotation $v^{+}>v^{-}$. Alors, pour tout nombre rationnel $\left.p / q \in\right] v^{-}, v^{+}[$écrit sous forme irrédutible, il existe une orbite périodique de période $q$ et de nombre de rotation $p / q$.

Remarquons que le résultat précédent est vrai plus généralement pour $v^{+}$ ou $v^{-}$dans $\mathbf{R} \cup\{-\infty,+\infty\}$. Comme autre application de l'existence de feuilletages transverses, nous allons donner une preuve rapide du résultat suivant, du à Franks [Fr5] :

Théorème 9.3. - Un homéomorphisme $\mathrm{F}$ de la sphère $\mathrm{S}^{2}$ qui préserve une mesure de probabilité de support $\mathrm{S}^{2}$ et qui a au moins trois points fixes a une infinité d'orbites périodiques. 
De plus si $\mathrm{F}$ préserve l'orientation et n'a qu'un nombre fini de points fixes, il existe des périodes arbitrairement grandes.

Démonstration. - Quitte à remplacer $\mathrm{F}$ par $\mathrm{F}^{2}$ dans le cas où $\mathrm{F}$ renverse l'orientation, il suffit de prouver que $\mathrm{F}$ a des points périodiques de périodes arbitrairement grandes si $\mathrm{F}$ préserve l'orientation et n'a qu'un nombre fini de points fixes. L'homéomorphisme est alors isotope à l'identité puisqu'il préserve l'orientation. Considérons un ensemble non enlacé maximal $\mathrm{X} \subset \mathrm{Fix}(\mathrm{F})$. C'est-à-dire supposons que $\mathrm{F}_{\mathrm{S}^{2} \backslash \mathrm{X}}$ soit isotope à l'identité et que $\mathrm{F}_{\mathrm{S}^{2} \backslash \mathrm{X} \cup\{z\}}$ ne le soit plus si $z \in \operatorname{Fix}(\mathrm{F}) \backslash \mathrm{X}$. Puisque $\mathrm{F}$ a au moins trois points fixes, $\mathrm{X}$ a au moins trois éléments. Pour tout $z \in \mathrm{S}^{2} \backslash \mathrm{X}$, la classe d'homotopie, à extrémités fixées, de l'arc $t \mapsto \mathrm{F}_{t}(z)$ est donc indépendante de l'isotopie $\left(\mathrm{F}_{t}\right)_{t \in[0,1]}$ qui joint l'identité à $\mathrm{F}$ en fixant les points de $\mathrm{X}$. Le théorème 8.1 nous donne l'existence d'un feuilletage $\mathscr{F}$ sur $\mathrm{S}^{2} \backslash \mathrm{X}$ transverse à l'isotopie.

Il n'y a pas de point errant, on sait donc d'après la proposition 8.2 que par tout point passe un lacet positivement transverse au feuilletage. Puisque nous nous trouvons sur une sphère, il n'y a donc pas de feuille fermée dans $\mathscr{F}$. Plus précisément, une telle feuille $\Gamma$ séparerait la sphère en deux parties ouvertes $\mathrm{U}_{d}$ et $\mathrm{U}_{g}$, la première à droite, la seconde à gauche de $\Gamma$ et on devrait avoir l'inclusion $\mathrm{F}\left(\overline{\mathrm{U}}_{d}\right) \subset \mathrm{U}_{d}$. Pour les mêmes raisons, on sait qu'il n'y a pas de cycle. Si on applique le théorème de Poincaré-Bendixson, on en déduit que toute feuille est une connexion entre deux points distincts.

Considérons une feuille $\phi$, issue d'un point $z_{0} \in \mathrm{X}$ et aboutissant en un point $z_{1} \in \mathrm{X}$. Notons $\widehat{\pi}: \widehat{\mathrm{A}} \rightarrow \mathrm{A}$ le revêtement universel de l'anneau ouvert $\mathrm{A}=\mathrm{S}^{2} \backslash\left\{z_{0}, z_{1}\right\}$. L'isotopie $\left(\mathrm{F}_{t \mid \mathrm{A}}\right)_{t \in[0,1]}$ se relève en une isotopie $\left(f_{t}\right)_{t \in[0,1]}$ sur $\widehat{\mathrm{A}}$ issue de l'identité. Puisque l'arc $t \mapsto \mathrm{F}_{t}(z)$ est homotope, à extrémités fixées, à un arc positivement transverse au feuilletage $\mathscr{F}$, on en déduit que l'arc $t \mapsto f_{t}(z)$ est homotope à un arc positivement transverse au feuilletage relevé $\widehat{\mathscr{F}}$ défini sur $\widehat{\mathrm{A}} \backslash \widehat{\pi}^{-1}\left(\mathrm{X} \backslash\left\{z_{0}, z_{1}\right\}\right)$. L'image par $\widehat{f}_{1}$ de toute composante connexe $\widehat{\phi}$ de $\widehat{\pi}^{-1}(\phi)$ est donc située à droite de $\widehat{\phi}$. Soit $\mathrm{U} \subset \mathrm{M} \backslash \mathrm{X}$ un petit disque ouvert proche et à droite de $\phi$ et $\left(\gamma_{z, z^{\prime}}\right)_{z, z^{\prime}}$ une famille d'arcs, à valeurs dans $\mathrm{U}$, joignant $z \in \mathrm{U}$ à $z^{\prime} \in \mathrm{U}$. Considérons l'ensemble $\mathrm{R}$ des points positivement récurrents, l'application de premier retour

$$
\begin{aligned}
\Phi: \mathrm{R} \cap \mathrm{U} & \rightarrow \mathrm{R} \cap \mathrm{U} \\
z & \mapsto \Phi(z)=\mathrm{F}^{\tau(z)}(z),
\end{aligned}
$$

et le lacet

$$
\Gamma_{z}=\left(\prod_{i=0}^{\tau(z)-1} \gamma_{\mathrm{F}^{i}(z)}\right) \gamma_{\Phi(z), z}
$$


défini pour $z \in \mathrm{R} \cap \mathrm{U}$. À cause de l'existence de $\widehat{\phi}$ et du choix de $\mathrm{U}$, on sait que

$$
m(z)=\Gamma_{z} \wedge \phi \geq 1
$$

On a

$$
\left[\Gamma_{z}\right]=m(z)\left[\Gamma^{*}\right]
$$

où $\Gamma^{*}$ est un lacet simple vérifiant $\Gamma^{*} \wedge \phi=1$. On a vu dans la section précédente que la suite

$$
\frac{1}{n} \sum_{i=0}^{n-1} \tau\left(\Phi^{i}(z)\right)
$$

converge presque surement sur $\mathrm{R} \cap \mathrm{U}$ dans $[1,+\infty[$ puisque $\tau$ est intégrable. Le théorème ergodique de Birkhoff nous dit également que la suite

$$
\frac{1}{n} \sum_{i=0}^{n-1} m\left(\Phi^{i}(z)\right)
$$

converge presque surement sur $\mathrm{R} \cap \mathrm{U}$ dans $[1,+\infty]$ puisque $m$ est à valeurs dans $[1,+\infty[$. Ainsi, la suite

$$
\left(\sum_{i=0}^{n-1} \tau\left(\Phi^{i}(z)\right)\right)^{-1}\left(\sum_{i=0}^{n-1} m\left(\Phi^{i}(z)\right)\right)
$$

converge presque surement sur $\mathrm{R} \cap \mathrm{U}$ dans ]0, $+\infty$ ]. En d'autres termes, presque tout point $z \in \mathrm{R} \cap \mathrm{U}$ a un nombre de rotation strictement positif (éventuellement infini). On sait d'autre part que $\mathrm{F}_{\mid \mathrm{A}}$ a au moins un point fixe de nombre de rotation zéro (tout point de $\mathrm{X} \backslash\left\{z_{0}, z_{1}\right\}$ convient). Le corollaire 9.2 appliqué à $\mathrm{F}_{\mid \mathrm{A}}$ nous donne alors l'existence d'orbites périodiques de $\mathrm{F}$ de périodes arbitrairement grandes.

Il existe d'autres versions de ce théorème. On sait par exemple d'après Handel [Hanl] que la conclusion est vraie si on remplace la propriété de préservation de la mesure par la propriété de transitivité topologique. Plus généralement, elle est vraie si on suppose qu'il n'y a pas de point errant [LeC4]. La méthode que l'on vient de donner permet également de retrouver ce cas plus général. En effet, la préservation de la mesure n'a été utilisée que dans la fin de la preuve pour construire des points de nombres de rotation non nuls. Dans le cas non errant, on peut conclure, à partir du fait que la feuille $\widehat{\phi}$ est envoyée sur sa droite et 
en utilisant le lemme de Franks sur les chaînes de disques libres, qu'il existe une infinité d'orbites périodiques (voir [LeC4]).

La démonstration originale de Franks du théorème 9.3 utilise le résultat suivant, attribué dans [Fr5] à Betsvina et Handel et qui est une conséquence du difficile théorème de point fixe de Handel [Han2]. Le théorème 8.1 permet de se passer du théorème de Handel ainsi que des arguments géométriques de la preuve de Betsvina-Handel (existence d'une métrique de courbure - 1, groupes fuchsiens, ...), seuls les arguments topologiques seront nécessaires.

Théorème 9.4. - Soit $\mathrm{M}$ une surface homéomorphe à une partie ouverte et connexe de la sphère (dont le complémentaire a au moins deux composantes connexes) et $\left(\mathrm{F}_{t}\right)_{t \in[0,1]}$ une isotopie dans $\mathrm{M}$ joignant l'identité à $\mathrm{F}$. On suppose que $\mathrm{F}$ n'a pas de point fixe contractile. Alors le nombre de rotation de toute orbite périodique de $\mathrm{F}$ est non nul.

Démonstration. - Considérons un feuilletage $\mathscr{F}$ sur $\mathrm{M}$ transverse à l'isotopie $\left(\mathrm{F}_{t}\right)_{t \in[0,1]}$ donné par le théorème 8.1. Si la conclusion du théorème est fausse, on peut trouver un lacet $\Gamma$ positivement transverse au feuilletage qui est homologue à zéro. Il existe alors une fonction $\Lambda: \mathrm{M} \backslash \Gamma \rightarrow \mathbf{Z}$ localement constante, uniquement définie par les propriétés suivantes :

$-\Lambda(z)=0$ si $z$ appartient à une composante connexe non relativement compacte de $\mathrm{M} \backslash \Gamma$;

$-\Lambda\left(z^{\prime}\right)-\Lambda(z)$ représente le nombre d'intersection algébrique $\Gamma^{\prime} \wedge \Gamma$ entre un arc $\Gamma^{\prime}$ joignant $z$ à $z^{\prime}$ et $\Gamma$.

Quitte à perturber $\Gamma$, on peut supposer que cet arc vérifie les propriétés suivantes :

- il n'a pas de point triple ou d'ordre supérieur ;

- il a un nombre fini de points doubles avec des auto-intersections transverses.

La fonction n'est pas constante et on doit avoir $\max \Lambda>0$ ou $\min \Lambda<0$. Plaçons nous dans le premier cas. Toute composante connexe $\mathrm{U}$ de $\mathrm{M} \backslash \Gamma$, où $\Lambda$ atteint son maximum est relativement compacte et c'est donc un disque puisque $\mathrm{M}$ est de genre nul. Pour les raisons indiquées dans la preuve de la proposition 9.1, sa frontière est une courbe fermée simple transverse au feuilletage. Ceci est bien sûr impossible puisque le feuilletage est non singulier, toujours par la formule de Poincaré-Hopf.

Remarquons que le théorème 9.4 est encore valable dans le cas de genre un (nous verrons qu'il cesse de l'être en genre supérieur ou égal à deux). En effet, si on suppose que la conclusion du théorème 9.4 n'est pas vérifiée et si on reprend la démonstration dans le cas où $\mathbf{M}$ est de genre un et non compacte, 
on remarque, dans le cas où $\max \Lambda>0$, que toute composante connexe $\mathrm{U}$ de $\mathrm{M} \backslash \Gamma$, où $\Lambda$ atteint son maximum est un anneau ouvert. Puisque $\Gamma$ est connexe, il n'y a qu'une composante et celle-ci est essentielle. Utilisant encore une fois la connexité de $\Gamma$, on en déduit que toute composante connexe de $M \backslash \Gamma$ où $\Lambda$ atteint son minimum est un disque (nécessairement) troué. Ainsi on a $\min \Lambda=0$. Le complémentaire de $\Lambda^{-1}(\{0\})$ est alors une surface à bord non vide de genre un, avec un feuilletage non singulier transverse au bord, car 0 est le minimum de $\Lambda$, on a bien une contradiction. Dans le cas où $M$ est une surface compacte de genre 1, il existe alors une fonction $\Lambda: M \backslash \Gamma \rightarrow \mathbf{Z}$ localement constante, définie à une constante près par la propriété suivante :

$-\Lambda\left(z^{\prime}\right)-\Lambda(z)$ représente le nombre d'intersection algébrique $\Gamma^{\prime} \wedge \Gamma$ entre un $\operatorname{arc} \Gamma^{\prime}$ joignant $z$ à $z^{\prime}$ et $\Gamma$.

Pour les raisons qui viennent d'être évoquées, toute composante connexe de $\mathrm{M} \backslash \Gamma$, où $\Lambda$ atteint son maximum ou son minimum est un anneau ouvert essentiel. L'ensemble $\mathrm{M} \backslash \Gamma$ possède donc au moins deux composantes connexes annulaires essentielles, ce qui est impossible puisque $\Gamma$ est connexe.

Construisons maintenant un contre-exemple en genre deux. Considérons une surface compacte de genre deux $\overline{\mathrm{M}}$ munie d'une structure symplectique et une fonction de Morse $\mathrm{L}$ sur $\overline{\mathrm{M}}$ ayant six points critiques $z_{1}, \ldots, z_{6}$, dont quatre selles, et six valeurs critiques distinctes $a_{1}<a_{2}<\ldots<a_{6}$. Nous construirons le contreexemple sur $\mathrm{M}=\overline{\mathrm{M}} \backslash\left\{z_{3}, z_{4}\right\}$. Fixons $\left.b_{2} \in\right] a_{2}, a_{3}\left[\right.$ et $\left.b_{4} \in\right] a_{4}, a_{5}\left[\right.$, notons $\mathrm{G}_{1}, \mathrm{C}_{2}$ les deux composantes connexes de $\mathrm{L}^{-1}\left(\left\{b_{2}\right\}\right)$ et $\mathrm{C}_{3}, \mathrm{C}_{4}$ les deux composantes connexes de $\mathrm{L}^{-1}\left(\left\{b_{4}\right\}\right)$. Fixons $\left.b_{2}^{\prime} \in\right] a_{2}, b_{2}\left[\right.$ et $\left.b_{4}^{\prime} \in\right] b_{4}, a_{5}$ [ et modifions le champ hamiltonien associé à $\mathrm{L}$ sur les anneaux fermés $\mathrm{L}^{-1}\left(\left[a_{1}, b_{2}^{\prime}\right]\right)$ et $\mathrm{L}^{-1}\left(\left[b_{4}^{\prime}, a_{6}\right]\right)$ pour créer deux composantes de Reeb et obtenir un champ de vecteurs $\xi$ sur $\bar{M}$ dont les points critiques $z_{3}$ et $z_{4}$ associés par L à $a_{3}$ et $a_{4}$ sont les seules singularités.

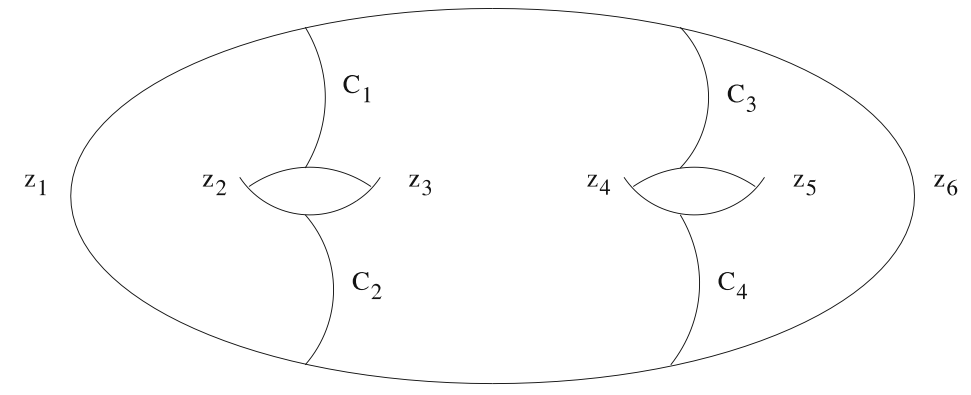

Le champ de vecteurs induit une orientation naturelle sur les cercles $\mathrm{C}_{i}$. Pour tous points distincts $z$ et $z^{\prime}$ de $\mathrm{C}_{i}$, notons $\left[z, z^{\prime}\right]_{i}$ le segment joignant $z$ à $z^{\prime}$ inclus dans $\mathrm{C}_{i}$ et positivement orienté. On choisit alors : 
- $z_{1,3}, z_{3,1}, z_{1,4}, z_{4,1}$ sur $\mathrm{C}_{1}$ cycliquement ordonnés par l'orientation naturelle de $\mathrm{G}_{1}$;

$-z_{2,4}, z_{4,2}$ sur $\mathrm{C}_{2}$;

$-z_{1,3}^{\prime}, z_{3,1}^{\prime}$ sur $\mathrm{C}_{3}$;

- $z_{1,4}^{\prime}, z_{4,1}^{\prime}, z_{4,2}^{\prime}, z_{2,4}^{\prime}$ sur $\mathrm{C}_{4}$ cycliquement ordonnés par l'orientation naturelle de $\mathrm{C}_{4}$.

On peut construire

- un segment orienté $\gamma_{1,3}$ joignant $z_{1,3}$ à $z_{1,3}^{\prime}$;

- un segment $\gamma_{3,1}$ joignant $z_{3,1}^{\prime}$ à $z_{3,1}$;

- un segment $\gamma_{1,4}$ joignant $z_{1,4}$ à $z_{1,4}^{\prime}$;

- un segment $\gamma_{4,1}$ joignant $z_{4,1}^{\prime}$ à $z_{4,1}$;

- un segment $\gamma_{2,4}$ joignant $z_{2,4}$ à $z_{2,4}^{\prime}$;

- un segment $\gamma_{4,2}$ joignant $z_{4,2}^{\prime}$ à $z_{4,2}$;

transverses au feuilletage de $\mathrm{M} \backslash\left\{z_{3}, z_{4}\right\}$ par les orbites de $\xi$ et donc à valeurs dans $\mathrm{L}^{-1}\left(\left[b_{2}, b_{4}\right]\right.$, tels que les ensembles

$$
\begin{aligned}
& \gamma_{1,3} \cup \gamma_{3,1} \cup\left[z_{1,3}, z_{3,1}\right]_{1} \cup\left[z_{1,3}^{\prime}, z_{3,1}^{\prime}\right]_{3}, \\
& \gamma_{1,4} \cup \gamma_{4,1} \cup\left[z_{1,4}, z_{4,1}\right]_{1} \cup\left[z_{1,4}^{\prime}, z_{4,1}^{\prime}\right]_{4}, \\
& \gamma_{2,4} \cup \gamma_{4,2} \cup\left[z_{4,2}, z_{2,4}\right]_{2} \cup\left[z_{4,2}^{\prime}, z_{2,4}^{\prime}\right]_{4},
\end{aligned}
$$

sont des cercles qui bordent dans $\overline{\mathrm{M}} \backslash\left\{z_{3}, z_{4}\right\}$ trois disques fermés disjoints inclus dans $\mathrm{L}^{-1}\left(\left[b_{2}, b_{4}\right]\right)$.

Quitte à multiplier le champ de vecteurs par une fonction strictement positive, on peut supposer, en notant $\left(\theta_{t}\right)_{t \in \mathbf{R}}$ le flot associé à $\xi$, que :

- les segments $\theta_{t}\left(\gamma_{i, j}\right), t \in[-1,1]$, sont disjoints deux à deux, pour tout $(i, j)$;

- les segments $\theta_{t}\left(\gamma_{i, j}\right)$ et $\theta_{t^{\prime}}\left(\gamma_{i^{\prime}, j^{\prime}}\right)$ sont disjoints, pour tous $t, t^{\prime}$ dans $[-1,1]$, si $(i, j) \neq\left(i^{\prime}, j^{\prime}\right)$;

- on a

$$
z_{3,1}^{\prime}=\theta_{3}\left(z_{1,3}^{\prime}\right), \quad z_{1,3}^{\prime}=\theta_{4}\left(z_{3,1}^{\prime}\right)
$$

- on a

$$
z_{1,4}=\theta_{4}\left(z_{3,1}\right), \quad z_{1,3}=\theta_{4}\left(z_{4,1}\right)
$$

- on a

$$
z_{4,1}^{\prime}=\theta_{3}\left(z_{1,4}^{\prime}\right), \quad z_{4,2}^{\prime}=\theta_{3}\left(z_{4,1}^{\prime}\right), \quad z_{1,4}^{\prime}=\theta_{3}\left(z_{2,4}^{\prime}\right)
$$

- on a

$$
z_{2,4}=\theta_{3}\left(z_{4,2}\right), \quad z_{4,2}=\theta_{4}\left(z_{2,4}\right) .
$$




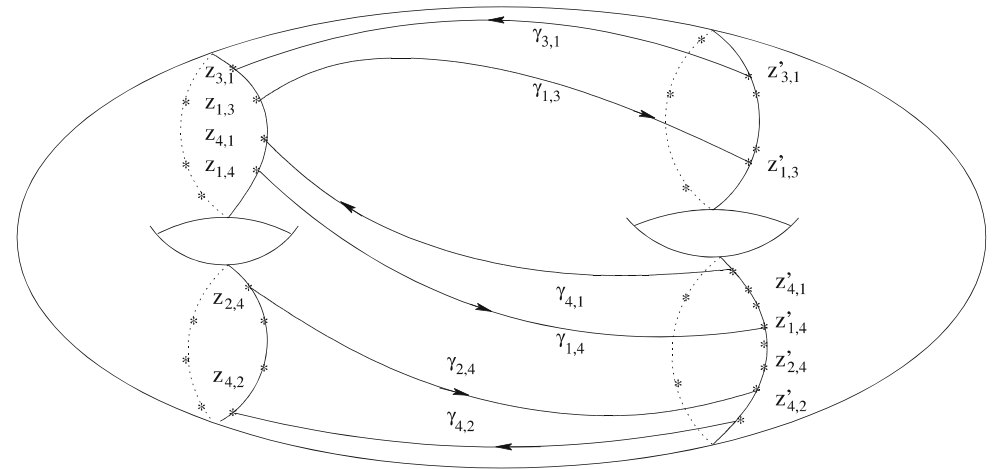

On se fixe maintenant un voisinage $\mathrm{U}_{i, j}$ de $\gamma_{i, j}$, en supposant les six voisinages disjoints deux à deux, et en supposant également que

$$
\mathrm{U}_{i, j} \cap \mathrm{L}^{-1}\left(\left[b_{2}, b_{4}\right]\right) \subset \bigcup_{-1 \leq t \leq 1} \theta_{t}\left(\gamma_{i, j}\right) .
$$

On considère alors une isotopie $\left(\mathrm{G}_{t}\right)_{t \in[0,1]}$, à support dans la réunion des six voisinages, telle que :

- l'arc $t \mapsto \mathrm{G}_{t}\left(z_{i, j}\right)$ est le segment $\gamma_{i, j}$, si $i \in\{1,2\}$;

- l'arc $t \mapsto \mathrm{G}_{t}\left(z_{i, j}^{\prime}\right)$ est le segment $\gamma_{i, j}$, si $i \in\{3,4\}$.

On définit ensuite une isotopie $\left(\mathrm{F}_{t}\right)_{t \in[0,1]}$ en posant :

$$
\begin{cases}\mathrm{F}_{t}=\theta_{4 t} & \text { si } t \in[0,1 / 2], \\ \mathrm{F}_{t}=\mathrm{G}_{2 t-1} \circ \theta_{2} & \text { si } t \in[1 / 2,1] .\end{cases}
$$

On vérifie que $z_{1,3}^{\prime}$ est un point périodique de période 20 de $\mathrm{F}_{1}$ et que l'arc $\prod_{0 \leq i \leq 19} \gamma_{\mathrm{F}^{i} z_{1,3}^{\prime}}$ est homologue dans $\mathrm{M}=\overline{\mathrm{M}} \backslash\left\{z_{3}, z_{4}\right\}$ à la somme des cycles $\mathrm{C}_{i}$ et donc homologue à zéro.

\section{Existence de points fixes pour les homéomorphismes hamiltoniens de surfaces}

Nous montrerons dans cette section l'assertion i) du théorème 1.5 énoncé en section 1 .

Théorème 10.1. - Soit $\mathrm{M}$ une surface compacte de genre $g \geq 1$ et $\left(\mathrm{F}_{t}\right)_{t \in[0,1]}$ une isotopie dans $\mathrm{M}$ joignant l'identité à un homéomorphisme $\mathrm{F}$. On suppose que $\mathrm{F}$ préserve une mesure borélienne de probabilité dont le support est $\mathrm{M}$ et que le nombre de rotation de cette mesure est nul. Il existe alors au moins trois points fixes contractiles. 
Démonstration. - On peut supposer, bien sûr, que l'ensemble $\mathrm{Fix}_{*}(\mathrm{~F}) \mathrm{des}$ points fixes contractiles de $\mathrm{F}$ est fini. On considère alors un ensemble non enlacé maximal $\mathrm{X} \subset \mathrm{Fix}_{*}(\mathrm{~F})$. On va montrer qu'il a au moins trois éléments. On se donne une isotopie $\left(\mathrm{F}_{t}^{\prime}\right)_{t \in[0,1]}$ de l'identité à $\mathrm{F}$ qui fixe tout point de $\mathrm{X}$ et telle que chaque arc $t \mapsto \mathrm{F}_{t}^{\prime}(z)$ est homotope à $t \mapsto \mathrm{F}_{t}(z)$ dans $\mathrm{M}$. On considère alors un feuilletage $\mathscr{F}$ sur $\mathrm{M} \backslash \mathrm{X}$ transverse à l'isotopie $\left(\mathrm{F}_{t}^{\prime}\right)_{t \in[0,1]}$. Pour tout $z \in \mathrm{M} \backslash \mathrm{X}$, on définit l'ensemble $\mathrm{C}(z) \subset \mathrm{H}_{1}(\mathbf{M}, \mathbf{Z})$ des classes d'homologie des lacets basés en $z$, inclus dans $\mathrm{M} \backslash \mathrm{X}$ et positivement transverses au feuilletage. Cet ensemble, qui est non vide d'après la proposition 8.2 iii), est clairement stable par addition.

Lemme 10.2. - Pour tout $z \in \mathrm{M} \backslash \mathrm{X}$, l'enveloppe convexe de $\mathrm{G}(z)$ est $\mathrm{H}_{1}(\mathrm{M}, \mathbf{R})$.

Démonstration. - Nous allons prouver qu'il existe $2 g+1$ lacets $\left(\Gamma_{i}\right)_{0 \leq i \leq 2 g}$ positivement transverses à $\mathscr{F}$ basés en $z$ et des entiers $\left(m_{i}\right)_{0 \leq i \leq 2 g}$ strictement positifs tels que :

- les classes d'homologie $\left[\Gamma_{i}\right]$ sont affinement indépendantes ;

- on a $\sum_{i=0}^{2 g} m_{i}\left[\Gamma_{i}\right]=0$.

Remarquons que si cette propriété est vraie pour un point $z$, elle est également vraie pour tout autre point $z^{\prime}$. En effet nous savons, d'après la proposition 8.2 iii), qu'il existe un arc $\gamma$ positivement transverse à $\mathscr{F}$ joignant $z$ à $z^{\prime}$ et un arc $\gamma^{\prime}$ joignant $z^{\prime}$ à $z$ ayant même propriété. Fixons $n \geq 1$ et posons $\Gamma_{i}^{\prime}=\gamma \Gamma_{i}^{n} \gamma^{\prime}$. Si $n$ est assez grand, les lacets $\Gamma_{i}^{\prime}$ verifierons les deux propriétés vérifiées par les $\Gamma_{i}$ qui viennent d'être citées. Plus généralement, l'adhérence dans $\mathrm{H}_{1}(\mathrm{M}, \mathbf{R})$ du cône positif engendré par $\mathrm{C}(z)$ est indépendante de $z$.

Pour montrer le lemme nous nous inspirerons de la preuve de [LeC2]. Nous allons raisonner par l'absurde et donc supposer que le lemme n'est pas vrai. Les ensembles $\mathrm{C}(z)$ sont alors tous contenus dans un même demi-espace fermé $\mathrm{E}^{+}$. On peut écrire

$$
\mathrm{E}^{+}=\left\{\rho \in \mathrm{H}_{1}(\mathrm{M}, \mathbf{R}) \mid \boldsymbol{\zeta} \cdot \rho \geq 0\right\},
$$

où $\zeta \in \mathrm{H}^{1}(\mathrm{M}, \mathbf{R})$ est non nul. Considérons le revêtement universel $\tilde{\pi}: \widetilde{\mathrm{M}} \rightarrow \mathrm{M}$ de $\mathrm{M}$ et notons $\mathrm{G}$ le groupe des automorphismes du revêtement. Considérons ensuite le morphisme de Hurewicz

$$
\begin{aligned}
\mathrm{G} & \rightarrow \mathrm{H}_{1}(\mathrm{M}, \mathbf{z}) \\
\mathrm{T} & \mapsto[\mathrm{T}],
\end{aligned}
$$

qui à $\mathrm{T} \in \mathrm{G}$ associe la classe d'homologie $[\mathrm{T}]=[\Gamma]$ de tout lacet $\Gamma$ qui est l'image par $\tilde{\pi}$ d'un arc joignant un point $z \in \widetilde{M}$ à $\mathrm{T} z$. Le sous-groupe

$$
\mathrm{H}=\{\mathrm{T} \in \mathrm{G} \mid \boldsymbol{\zeta} \cdot[\mathrm{T}]=0\}
$$


est distingué, et on a un revêtement naturel $\widehat{\pi}: \widehat{M}=\mathrm{M} / \mathrm{H} \rightarrow \mathrm{M}$ dont le groupe des automorphismes est $\mathrm{G} / \mathrm{H}$. On peut définir naturellement $\varsigma .[\mathrm{T}]$ pour $\mathrm{T} \in \mathrm{G} / \mathrm{H}$. L'isotopie $\left(\mathrm{F}_{t}^{\prime}\right)_{t \in[0,1]}$ se relève en une isotopie $\left(\widehat{\mathrm{F}}_{t}\right)_{t \in[0,1]}$ joignant l'identité à un relèvement $\widehat{\mathrm{F}}$ de $\mathrm{F}$. Le feuilletage relevé $\widehat{\mathscr{F}}$ défini sur $\widehat{\mathrm{M}} \backslash \widehat{\pi}^{-1}(\mathrm{X})$ est transverse à l'isotopie $\left(\widehat{\mathrm{F}}_{t}\right)_{t \in[0,1]}$. Remarquons que si $\mathrm{T} \in \mathrm{G} / \mathrm{H}$ vérifie $\varsigma$.[T] $<0$, il n'existe aucun arc positivement transverse à $\widehat{\mathscr{F}}$ joignant un point $z \in \widehat{M} \backslash \widehat{\pi}^{-1}(\mathrm{X})$ à $\mathrm{T} z$. En d'autres termes, le feuilletage $\widehat{\mathscr{F}}$ ne vérifie pas l'assertion iii) de la proposition 8.2. En conséquence, $\widehat{\mathrm{F}}$ possède des points errants.

Considérons un disque ouvert $\mathrm{U}$ tel que le revêtement $\widehat{\pi}: \widehat{\pi}^{-1}(\mathrm{U}) \rightarrow \mathrm{U}$ soit trivial, et tel que toute composante connexe de $\widehat{\pi}^{-1}(\mathrm{U})$ soit errante. Fixons une composante connexe $\widehat{\mathrm{U}}$ de $\widehat{\pi}^{-1}(\mathrm{U})$. Presque tout point $z \in \mathrm{U}$ étant récurrent, on note $\Phi: \mathrm{U} \rightarrow \mathrm{U}$ l'application de premier retour. Pour tout point récurrent $z \in \mathrm{U}$, on peut définir le temps de premier retour $\tau(z)$ dans $U$ de l'orbite positive de $z$. Si $\widehat{z}$ est l'antécédent de $z$ qui est dans $\widehat{\mathrm{U}}$, il existe $\mathrm{T}_{z} \in \mathrm{G} / \mathrm{H}$ tel que $\widehat{\mathrm{F}}^{\tau(z)} \in$ $\mathrm{T}_{z}(\widehat{\mathrm{U}})$. L'application $\theta: z \mapsto \varsigma \cdot\left[\mathrm{T}_{z}\right]$ est $\mu_{\mid \mathrm{U}}$-intégrable puisque $\theta / \tau$ est bornée, et strictement positive puisque $\widehat{\mathrm{U}}$ est errant. Les limites $\tau^{*}, \theta^{*}$ des moyennes de Birkhoff de $\tau$ et $\theta$ sont presque surement finies et strictement positives et on a $\varsigma \cdot \rho(z)=\theta^{*}(z) / \tau^{*}(z)$, où $\rho(z)$ est le vecteur de rotation de $z$. Ainsi $\varsigma . \rho(z)>0$ pour presque tout $z \in \mathrm{U}$.

Pour obtenir la contradiction, nous utilisons l'assertion ii) de la proposition 8.2 , qui implique que $\varsigma . \rho(z) \geq 0$ pour presque tout point $z \in$ M. Puisque

$$
\varsigma \cdot \rho(\mu)=\int_{\mathrm{M}} \varsigma \cdot \rho(z) d \mu=0,
$$

on en déduit que la fonction $z \mapsto \varsigma . \rho(z)$ est presque surement nulle sur M.

$$
\text { Lemme 10.3. - On a l'égalité } \mathrm{G}(z)=\mathrm{H}_{1}(\mathrm{M}, \mathbf{Z}) \text { pour tout } z \in \mathrm{M} \backslash \mathrm{X} \text {. }
$$

Démonstration. - Montrons d'abord que $\mathrm{G}(z)$ est un sous groupe de $\mathrm{H}_{1}(\mathrm{M}, \mathbf{Z})$. Il suffit de prouver que $-a \in \mathrm{G}(z)$ pour tout $a \in \mathrm{G}(z)$. Or on sait d'après le lemme précédent que $-a$ est barycentre à coefficients rationnels positifs d'éléments de $\mathrm{C}(z)$. On en déduit qu'il existe un entier $m>0$ tel que $-m a \in \mathrm{C}(z)$. On écrit alors

$$
-a=-m a+(m-1) a \in \mathrm{C}(z) .
$$

D'après le lemme 10.2, on sait que $\mathrm{G}(z)$ est un réseau de $\mathrm{H}_{1}(\mathrm{M}, \mathbf{z})$ et que $\mathrm{H}_{1}(\mathrm{M}, \mathbf{Z}) / \mathrm{C}(z)$ est donc fini. Le sous-groupe

$$
\mathrm{H}=\{\mathrm{T} \in \mathrm{G} \mid[\mathrm{T}] \in \mathrm{C}(z)\}
$$


est distingué, et on a un revêtement naturel $\widehat{\pi}: \widehat{M}=M / H \rightarrow M$ dont le groupe des automorphismes $\mathrm{G} / \mathrm{H}$ est fini, isomorphe à $\mathrm{H}_{1}(\mathrm{M}, \mathbf{Z}) / \mathrm{C}(z)$. Notons $\widehat{\mathscr{F}}$ le feuilletage relevé défini sur $\widehat{\mathrm{M}} \backslash \widehat{\pi}^{-1}(\mathrm{X})$. Il est transverse à l'isotopie relevée $\left(\widehat{\mathrm{F}}_{t}\right)_{t \in[0,1]}$ issue de l'identité. Nous allons montrer que le relèvement $\widehat{\mathrm{F}}_{1}$ n'a pas de point errant. Puisque $F$ n'a pas de point errant et puisque $\mathrm{G} / \mathrm{H}$ est fini, nous savons que pour toute partie ouverte non vide $\mathrm{U}$ de $\widehat{\mathrm{M}}$, il existe au moins un automorphisme $\mathrm{T} \in \mathrm{G} / \mathrm{H}$ tel que la suite $\left(\widehat{\mathrm{F}}_{1}^{n}(\mathrm{U})\right)_{n \geq 1}$ rencontre $\mathrm{T}(\mathrm{U})$ une infinité de fois. Notons $\mathscr{T}_{\mathrm{U}}$ l'ensemble des automorphismes vérifiant cette propriété et notons $l_{\mathrm{U}}$ le cardinal de $\mathscr{T}_{\mathrm{U}}$. Remarquons que $\mathscr{T}_{\mathrm{U}}=\mathscr{T}_{\hat{\mathrm{F}}_{1}(\mathrm{U})}$ puisque $\mathrm{F}$ commute avec les automorphismes. Nous voulons montrer que $\operatorname{Id}_{\widehat{M}} \in \mathscr{T}_{U}$ pour toute partie ouverte non vide $\mathrm{U}$. Si tel n'est pas le cas, il existe une partie ouverte non vide $\mathrm{U}_{0}$ telle $\operatorname{Id}_{\widehat{M}} \notin \mathscr{T}_{\mathrm{U}_{0}}$ et telle que $l_{\mathrm{U}} \geq l_{\mathrm{U}_{0}}$ si $\operatorname{Id}_{\widehat{M}} \notin \mathscr{T}_{\mathrm{U}}$. Fixons $\mathrm{T} \in \mathscr{T}_{\mathrm{U}_{0}}$ et $n \geq 1$ tel que $\mathrm{U}_{1}=\widehat{\mathrm{F}}_{1}^{n}\left(\mathrm{U}_{0}\right) \cap \mathrm{T}\left(\mathrm{U}_{0}\right) \neq \emptyset$. Remarquons que $\mathscr{T}_{\mathrm{U}_{1}} \subset \mathscr{T}_{\mathrm{F}_{1}^{n}}\left(\mathrm{U}_{0}\right) \subset \mathscr{T}_{\mathrm{U}_{0}}$ et montrons que $\mathrm{T} \notin \mathscr{T}_{\mathrm{U}_{1}}$, ce qui donnera une contradiction. Or si $m$ est assez grand,

$$
\begin{aligned}
\widehat{\mathrm{F}}_{1}^{m}\left(\mathrm{U}_{1}\right) \cap \mathrm{T}\left(\mathrm{U}_{1}\right) & =\widehat{\mathrm{F}}_{1}^{n+m}\left(\mathrm{U}_{0}\right) \cap \mathrm{T}\left(\widehat{\mathrm{F}}_{1}^{m}\left(\mathrm{U}_{0}\right)\right) \cap \mathrm{T}\left(\widehat{\mathrm{F}}_{1}^{n}\left(\mathrm{U}_{0}\right)\right) \cap \mathrm{T}^{2}\left(\mathrm{U}_{0}\right) \\
& \subset \mathrm{T}\left(\widehat{\mathrm{F}}_{1}^{m}\left(\mathrm{U}_{0}\right) \cap \widehat{\mathrm{F}}_{1}^{n}\left(\mathrm{U}_{0}\right)\right)
\end{aligned}
$$

est vide puisque $\operatorname{Id}_{\widehat{M}} \notin \mathscr{T}_{\mathrm{U}_{0}}$. Puisque $\widehat{\mathrm{F}}_{1}$ n'a pas de point errant, on peut appliquer l'assertion iii) du théorème 8.2 à $\widehat{\mathscr{F}}$. On en déduit que $\mathrm{H}=\mathrm{G}$ puisque pour tout élément non nul $\mathrm{T} \in \mathrm{G} / \mathrm{H}$, il n'existe aucun arc positivement transverse à $\widehat{\mathscr{F}}$ joignant un point $z \in \widehat{\mathrm{M}}$ à $\mathrm{T} z$.

Suite de la démonstration du théorème 10.1. - On se fixe un point base $z_{*} \in \mathrm{M} \backslash \mathrm{X}$ dans cette démonstration et même jusqu'à la fin de la section 10. Grâce au lemme 10.3 , on peut trouver une famille $\left(\Gamma_{i}\right)_{0 \leq i \leq n}$ de lacets positivement transverses à $\mathscr{F}$ basés en $z_{*}$, et en particulier disjoints de $\mathrm{X}$, tels que tout élément $\kappa \in \mathrm{H}_{1}(\mathrm{M}, \mathbf{Z})$ s'écrit $\kappa=\sum_{i=0}^{n} l_{i}\left[\Gamma_{i}\right]$, avec $l_{i}>0$.

En particulier, il existe des entiers $m_{i}>0$ tels que le lacet $\Gamma=\prod_{i=0}^{n} \Gamma_{i}^{m_{i}}$ est homologue à zéro. On peut toujours supposer :

- que chaque $\Gamma_{i}$ n'a pas de point triple ou d'ordre supérieur;

- que chaque $\Gamma_{i}$ a un nombre fini de points doubles avec des auto-intersections transverses;

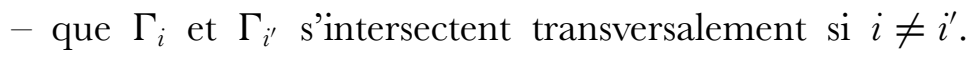

On peut définir alors une fonction $\Lambda: \mathrm{M} \backslash \Gamma \rightarrow \mathbf{Z}$ localement constante, telle que $\Lambda\left(z^{\prime}\right)-\Lambda(z)=\Gamma^{\prime} \wedge \Gamma$, pour tout arc $\Gamma^{\prime}$ joignant $z$ à $z^{\prime}$. On note $\min \Lambda$ (resp. $\max \Lambda$ ) la valeur minimale (resp. maximale) prise par cette fonction. Montrons que toute composante connexe $\mathrm{U}$ de $\mathrm{M} \backslash \Gamma$ est un disque ouvert. L'application

$$
\iota_{*}: \mathrm{H}_{1}(\mathrm{U}, \mathbf{Z}) \rightarrow \mathrm{H}_{1}(\mathrm{M}, \mathbf{Z})
$$


induite par l'inclusion

$$
\iota: \mathrm{U} \hookrightarrow \mathrm{M}
$$

est nulle. En effet, puisque pour tout $\kappa \in \mathrm{H}_{1}(\mathrm{U}, \mathbf{Z})$ et pour tout $i \in\{0, \ldots, n\}$, on a $\iota_{*}(\kappa) \wedge \Gamma_{i}=0$, on sait que $\iota_{*}(\kappa) \wedge \kappa^{\prime}=0$ pour tout $\kappa^{\prime} \in \mathrm{H}_{1}(\mathrm{M}, \mathbf{Z})$ et donc que $\iota_{*}(\kappa)=0$. Comme la réunion des $\Gamma_{i}$ est connexe, on en déduit que $\mathrm{H}_{1}(\mathrm{U}, \mathbf{Z})=0$ et donc que $\mathrm{U}$ est un disque ouvert, comme annoncé.

Si $U$ est une composante connexe de $\Lambda^{-1}(\{\min \Lambda\})$, son adhérence est un disque fermé et la frontière, qui est une variété, est transverse au feuilletage, il y a donc au moins un point de $\mathrm{X}$ dans $\mathrm{U}$. De même, il y au moins un point de $\mathrm{X}$ dans chaque composante connexe de $\Lambda^{-1}(\{\max \Lambda\})$. Plus précisément, si on note $i(\mathscr{F}, z)$ l'indice du feuilletage $\mathscr{F}$ en la singularité $z \in \mathrm{X}$, on sait que pour toute composante connexe $\mathrm{U}$ de $\mathrm{M} \backslash \Gamma$ où $\Lambda$ atteint son minimum ou son maximum, on a

$$
\sum_{z \in \mathrm{X} \cap \mathrm{U}} i(\mathscr{F}, z)=1
$$

Grâce à la formule de Poincaré-Hopf

$$
\sum_{z \in \mathrm{X}} i(\mathscr{F}, z)=2-2 g \leq 0
$$

on sait qu'il existe au moins un point $z \in \mathrm{X}$ tel que

$$
\min \Lambda<\Lambda(z)<\max \Lambda \text {. }
$$

Il y a donc au moins trois points dans $\mathrm{X}$.

On montrera dans la prochaine section l'assertion ii) du théorème 1.5 , à savoir l'existence d'une infinité de points périodiques contractiles. Il nous faut pour cela préciser la dynamique du feuilletage $\mathscr{F}$, ce qui va être fait dans le reste de cette section. On verra qu'elle est de type gradient. On suppose donc que F vérifie les hypothèses du théorème 10.1 et que l'ensemble $\mathrm{Fix}_{*}(\mathrm{~F})$ des points fixes contractiles de $\mathrm{F}$ est fini. On se fixe une partie non enlacée maximale $\mathrm{X} \subset \mathrm{Fix}_{*}(\mathrm{~F})$ et un feuilletage transverse $\mathscr{F}$ sur $\mathrm{M} \backslash \mathrm{X}$. Rappelons qu'on a construit une famille $\left(\Gamma_{i}\right)_{0 \leq i \leq n}$ de lacets positivement transverses à $\mathscr{F}$ basés en un point $z_{*}$, tels que

- chaque $\Gamma_{i}$ n'a pas de point triple ou d'ordre supérieur ;

- chaque $\Gamma_{i}$ a un nombre fini de points doubles avec des auto-intersections transverses ;

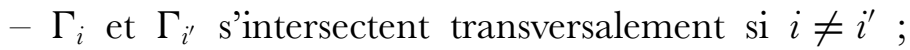

- tout élément $\kappa \in \mathrm{H}_{1}(\mathrm{M}, \mathbf{Z})$ s'écrit $\kappa=\sum_{i=0}^{n} l_{i}\left[\Gamma_{i}\right]$, avec $l_{i}>0$. 
En particulier, il existe des entiers $m_{i}>0$ tels que le lacet $\Gamma=\prod_{i=0}^{n} \Gamma_{i}^{m_{i}}$ est homologue à zéro et on a défini une fonction $\Lambda: M \backslash \Gamma \rightarrow \mathbf{Z}$ localement constante, telle que $\Lambda\left(z^{\prime}\right)-\Lambda(z)=\Gamma^{\prime} \wedge \Gamma$, pour tout arc $\Gamma^{\prime}$ joignant $z$ à $z^{\prime}$.

On dira qu'une singularité $z \in \mathrm{X}$ est un puits (resp. une source) si le feuilletage est équivalent dans un voisinage de $z$ au feuilletage du plan formé des demi-droites dirigées vers le centre (resp. vers l'extérieur). On dira que $z$ est un point selle si $\mathscr{F}$ est équivalent au voisinage de $z$ à un feuilletage sur le plan formé de $p$ secteurs, réunions de demi-droites dirigées vers le centre, éventuellement réduits à une demi-droite, alternant avec $p$ secteurs réunions de demi-droites dirigées vers l'extérieur et séparant des secteurs hyperboliques. Pour chaque entier $p$, on obtient un nombre fini de modèles possibles. Remarquons que pour chacun d'eux, l'indice $i(\mathscr{F}, z)$ du feuilletage en $z$ est égal à $1-p$. Nous noterons $i(\mathrm{~F}, z)$ l'indice de Lefschetz du point fixe $z$ de F.
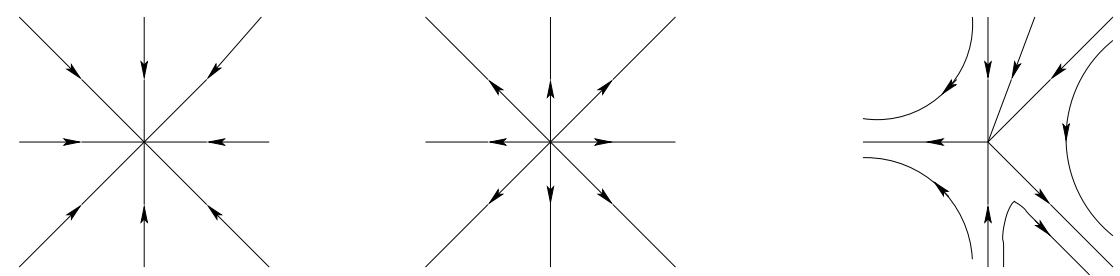

Pour tout $z \in \mathrm{M} \backslash \mathrm{X}$, on notera $\phi_{z}$ la feuille contenant $z, \phi_{z}^{+}$la demi-feuille positive issue de $z, \phi_{z}^{-}$la demi-feuille négative aboutissant à $z$. Toute composante connexe $\mathrm{U}$ de $\mathrm{M} \backslash \Gamma$ est un bloc isolant du feuilletage au sens de Conley [Co] : toute feuille contenue dans $\overline{\mathrm{U}}$ doit être contenue dans $\mathrm{U}$ et même relativement compacte dans U. Plus précisément, puisque $\Lambda$ décroit le long d'une feuille, trois cas sont possibles, si $z \in \partial \mathrm{U}$ :

- la demi-feuille $\phi_{z}^{-}$ne rencontre $\overline{\mathrm{U}}$ qu'au point $z$, contrairement à $\phi_{z}^{+}$, on dira que $z$ est un point rentrant ;

- la feuille $\phi_{z}^{+}$ne rencontre $\overline{\mathrm{U}}$ qu'au point $z$, contrairement à $\phi_{z}^{-}$, on dira que $z$ est un point sortant ;

- la feuille $\phi_{z}$ ne rencontre $\overline{\mathrm{U}}$ qu'au point $z$.

On notera $\partial^{+} \mathrm{U}$ l'ensemble des points rentrant, $\partial^{-} \mathrm{U}$ l'ensemble des points sortant et $\partial^{ \pm} \mathrm{U}$ l'ensemble des points qui ne sont ni rentrant ni sortant.

$\mathrm{Si}$ on rajoute à notre famille $\left(\Gamma_{i}\right)_{0 \leq i \leq n}$ un nombre fini de lacets basés en $z_{*}$ et positivement transverses à $\mathscr{F}$, la famille augmentée vérifie les mêmes propriétés que la famille d'origine et on peut construire un lacet $\Gamma$ et une fonction $\Lambda$ associée décrivant une décomposition en ensembles isolants plus fine que celle d'origine. Utilisant ce principe, nous démontrerons : 
Proposition 10.4. - Le feuilletage $\mathscr{F}$ vérifie les propriétés suivantes :

i) il n'y a pas de feuille fermée ni de cycle de connexions;

ii) toute feuille définit une connexion;

iii) tout point $z \in \mathrm{X}$ est un puits, une source ou une selle de $\mathscr{F}$;

iv) pour toute selle $z \in \mathrm{X}$, on a $i(\mathrm{~F}, z)=i(\mathscr{F}, z)$;

v) si $\mathrm{X}=\mathrm{Fix}_{*}(\mathrm{~F})$, alors pour toute source ou puits $z \in \mathrm{X}$, on a $i(\mathrm{~F}, z)=i(\mathscr{F}, z)$ $=1$.

\section{Démonstration.}

Preuve de i). - Supposons que $\Gamma^{\prime}$ soit une feuille fermée et fixons $z \in \Gamma^{\prime}$. L'homéomorphisme $\mathrm{F}$ n'ayant pas de point errant, nous pouvons utiliser la proposition 8.2 et construire un lacet $\Gamma^{\prime \prime}$ basé en $z$ et positivement transverse à $\mathscr{F}$. Nous avons donc $\Gamma^{\prime} \wedge \Gamma^{\prime \prime}<0$. Puisqu'il existe des entiers $l_{i}>0$ tels que

$$
-\left[\Gamma^{\prime \prime}\right]=\sum_{i=0}^{n} l_{i}\left[\Gamma_{i}\right],
$$

nous avons

$$
0>\Gamma^{\prime} \wedge \Gamma^{\prime \prime}=-\sum_{i=0}^{n} l_{i} \Gamma^{\prime} \wedge \Gamma_{i} \geq 0 .
$$

Nous avons obtenu une contradiction. On démontre pour les mêmes raisons qu'il n'y a pas de cycle de connexions. En fait, puisque toute feuille intersecte un lacet positivement transverse, on peut toujours supposer, quitte à complèter notre famille $\left(\Gamma_{i}\right)_{0 \leq i \leq n}$, qu'elle vérifie la propriété supplémentaire suivante, ce que l'on supposera désormais :

- s'il existe une connexion de $z_{0} \in \mathrm{X}$ à $z_{1} \in \mathrm{X}$, l'un au moins des lacets $\Gamma_{i}$ rencontre une connexion de $z_{0}$ à $z_{1}$.

Ainsi s'il y a une connexion de $z_{0} \in \mathrm{X}$ à $z_{1} \in \mathrm{X}$, on a $\Lambda\left(z_{1}\right)<\Lambda\left(z_{0}\right)$, en particulier il n'y a pas de cycle.

Preuve de ii). - Si U est une composante connexe de $\mathrm{M} \backslash \Gamma$, il n'y a pas de feuille contenue dans $\bar{U}$. En effet une telle feuille doit être contenue dans U et relativement compacte. Puisque $U$ est un disque ouvert, le théorème de PoincaréBendixson implique alors l'existence dans U d'une feuille fermée ou d'une feuille connectant deux points (éventuellement égaux) de $\mathrm{X} \cap \mathrm{U}$, ce qui est impossible. Pour les mêmes raisons, toute demi-feuille positive contenue dans $\overline{\mathrm{U}}$ aboutit à un point $z \in \mathrm{X} \cap \mathrm{U}$ et toute demi-feuille négative est issue d'un point $z \in \mathrm{X} \cap \mathrm{U}$. 
Ainsi, la fonction $\Lambda$ est définie sur toute feuille $\phi$, à l'exception d'un ensemble fini non vide de points, elle est décroissante avec des sauts en chacun des points précédents. Toute feuille $\phi$ joint un point $\alpha(\phi) \in \mathrm{X}$ à un point $\omega(\phi) \in \mathrm{X}$ vérifiant $\Lambda(\omega(\phi))<\Lambda(\alpha(\phi))$, elle définit nécessairement une connexion. La dynamique du feuilletage est celle d'un champ de gradient. La topologie restreinte à une feuille $\phi$ est celle de toute paramétrage de $\phi$, on parlera naturellement d'intervalle de $\phi$, ouvert ou fermé, de segment, de demi-droite positive ou négative, de demi-droite ouverte ou fermée.

Preuve de iii). - Toute composante $\mathrm{U}$ de $\mathrm{M} \backslash \Gamma$ est un disque ouvert. Par contre, il se peut que son adhérence ne soit pas un disque fermé dans le cas où les ensembles $\partial^{+} \mathrm{U}$ et $\partial^{-} \mathrm{U}$ sont tous deux non vides. Quitte à augmenter notre famille $\left(\Gamma_{i}\right)_{0 \leq i \leq n}$, on verra qu'on peut toujours supposer que $\overline{\mathrm{U}}$ est un disque et qu'il y a au plus une singularité dans $U$. Le cas où $\bar{U}$ n'est pas un disque correspond au cas où sa frontière n'est pas une sous-variété de dimension un. Il existe alors un point $z \in \partial \mathrm{U}$ qui est point double d'un lacet $\Gamma_{i}$ ou point d'intersection de deux lacets $\Gamma_{i}$ et $\Gamma_{i^{\prime}}$ où $m_{i}=m_{i^{\prime}}$, et tel que $z$ est adhérent à exactement deux autres composantes connexes $\mathrm{U}_{-}, \mathrm{U}_{+}$de $\mathrm{M} \backslash \Gamma$ avec :

$$
\Lambda\left(\mathrm{U}_{-}\right)=\Lambda(\mathrm{U})-m_{i}, \quad \Lambda\left(\mathrm{U}_{+}\right)=\Lambda(\mathrm{U})+m_{i}
$$

Dans un voisinage de $z$, on choisit un arc $\gamma$ joignant un point $z_{+} \neq z$ de $\partial \mathrm{U}_{+}$à un point $z_{-} \neq z$ de $\partial U_{-}$, à valeurs, sauf aux extrémités, dans $\mathrm{U}$ et positivement transverse à $\mathscr{F}$. Il suffit pour cela de perturber un segment de feuille issu de $\partial \mathrm{U}_{+}$et aboutissant en $\partial \mathrm{U}_{-}$. Si $\Gamma_{i_{+}}\left(\operatorname{resp} . \Gamma_{i_{-}}\right)$est le lacet contenant $z_{+}$(resp. $\left.z_{-}\right)$, on note $\gamma_{i_{+}}$le sous-arc de $\Gamma_{i_{+}}$qui joint le point base $z_{*}$ à $z_{+}$et $\gamma_{i_{-}}$le sous-arc de $\Gamma_{i_{-}}$qui joint $z_{-}$à $z_{*}$. L'arc $\Gamma_{z}=\gamma_{i_{+}} \gamma \gamma_{i_{-}}$est un lacet positivement transverse à $\mathscr{F}$. Quitte à rajouter à notre famille $\left(\Gamma_{i}\right)_{0 \leq i \leq n}$, tous les lacets $\Gamma_{z}$ ainsi construits, à modifier les coefficients $m_{i}$, et à perturber les lacets $\Gamma_{z}$ pour qu'ils soient en position générale, on peut toujours supposer que l'adhérence de toute composante connexe de $\mathrm{M} \backslash \Gamma$ est un disque.

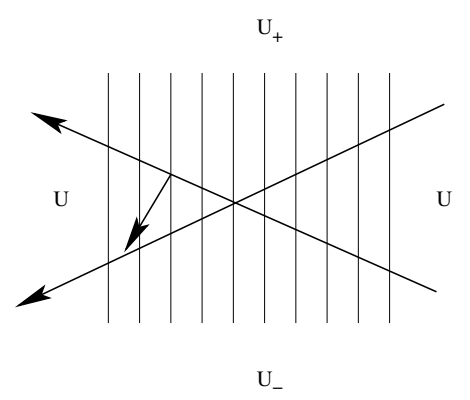


La trace $\phi \cap \overline{\mathrm{U}}$ d'une feuille $\phi$ sur $\overline{\mathrm{U}}$, si elle n'est pas vide, est une demidroite positive $\phi_{z}^{+}, z \in \partial^{+}(\mathrm{U})$; une demi-droite négative $\phi_{z}^{-}, z \in \partial^{-}(\mathrm{U})$; un segment joignant un point de $\partial^{+}(\mathrm{U})$ à un point de $\partial^{-}(\mathrm{U})$; ou se réduit à un point $z \in \partial^{ \pm}(\mathrm{U})$. S'il existe un segment $\phi \cap \overline{\mathrm{U}}$ qui sépare dans $\overline{\mathrm{U}}$ deux points de $\mathrm{X} \cap \mathrm{U}$, on peut le perturber pour construire un arc ouvert positivement transverse à $\mathscr{F}$ joignant dans $\mathrm{U}$ un point de $\partial^{+} \mathrm{U}$ à un point de $\partial^{-} \mathrm{U}$. On peut, comme précédemment, l'étendre en un lacet positivement transverse basé en $z^{*}$. Ainsi, quitte à rajouter des lacets à nos $\Gamma_{i}$, on peut supposer que la trace d'une feuille sur l'adhérence d'une composante $\mathrm{U}$ de $\mathrm{M} \backslash \Gamma$ ne sépare jamais dans $\overline{\mathrm{U}}$ deux points de $\mathrm{X} \cap \mathrm{U}$.

Nous allons voir que ceci implique qu'il y a au plus un point de $\mathrm{X}$ dans chaque composante et que la dynamique peut être décrite très précisément à l'intérieur d'une composante. Les résultats qui suivent sont très classiques.

Soit U une composante connexe contenant des points sortant et des points rentrant. On peut écrire $\partial \mathrm{U}=\prod_{l \in \mathbf{Z} / 2 q \mathbf{Z}} \gamma_{l}$, où chaque $\gamma_{l}$ est un arc dont les extrémités sont sur $\partial^{ \pm} \mathrm{U}$ et dont les autres points sont sur $\partial^{+} \mathrm{U}$ si $l$ est pair et sur $\partial^{-} \mathrm{U}$ si $l$ est impair. Supposons d'abord $q \geq 2$. Notons $\breve{\gamma}_{l}$ l'arc ouvert obtenu à partir de $\gamma_{l}$ en otant ses extrémités et munissons cet ensemble de l'ordre naturel défini par paramétrage. Fixons $l$ pair. Si $z$ appartient à $\breve{\gamma}_{l}$ et si $\phi_{z} \cap \overline{\mathrm{U}}$ est un segment, celui-ci aboutit en un point $z^{\prime} \in \breve{\gamma}_{l^{\prime}(z)}$, où $l^{\prime}(z)$ est impair. L'ensemble $\mathrm{I}_{l}$ des points $z \in \breve{\gamma}_{l}$ tels que $\phi_{z} \cap \overline{\mathrm{U}}$ est un segment est donc ouvert dans $\breve{\gamma}_{l}$ et l'application $z \mapsto l^{\prime}(z)$ localement constante. Si $z$ est proche de l'extrémité initiale (resp. finale) de $\gamma_{l}$, il appartient à $\mathrm{I}_{l}$ et $l^{\prime}(z)=l-1\left(\operatorname{resp} . l^{\prime}(z)=l+1\right)$. On en déduit que $\mathrm{I}_{l} \neq \breve{\gamma}_{l}$ et on peut définir

$$
z_{l}^{-}=\inf \breve{\gamma}_{l} \backslash \mathrm{I}_{l}, \quad z_{l}^{+}=\sup \breve{\gamma}_{l} \backslash \mathrm{I}_{l} .
$$

Remarquons qu'aucun point $z$ de $\breve{\gamma}_{l}$ situé entre $z_{l}^{-}$et $z_{l}^{+}$n'appartient à $\mathrm{I}_{l}$. En effet, le segment $\phi_{z} \cap \overline{\mathrm{U}}$ séparerait alors dans $\overline{\mathrm{U}}$ les deux points $\omega\left(\phi_{z_{l}^{-}}\right)$et $\omega\left(\phi_{z_{l}^{+}}\right)$.

Remarquons que l'application $z \mapsto \omega\left(\phi_{z}\right)$ est localement constante, et donc constante, sur le segment $\breve{\gamma}_{l} \backslash \mathrm{I}_{l}$. En effet, si $\left(z_{n}\right)_{n \geq 0}$ est une suite dans $\breve{\gamma}_{l} \backslash \mathrm{I}_{l}$ convergeant vers $z$ telle que $\omega\left(\phi_{z}\right)=z^{*} \in \mathrm{X}$ et si $\mathrm{K}$ est une valeur d'adhérence, pour la topologie de Hausdorff, de la suite $\overline{\phi_{z_{n}}^{+}}$alors $\mathrm{K}$ contient $\overline{\phi_{z}^{+}}$et $z^{*}$. Il ne rencontre aucune feuille autre que $\phi_{z}$, puique $\overline{\mathrm{U}}$ ne contient aucune feuille. Ainsi, $\mathrm{K}$ est formé de $\overline{\phi_{z}^{+}}$et de points de $\mathrm{X}$. Puisque $\mathrm{K}$ est connexe et $\mathrm{X}$ est fini, $\mathrm{K}$ se réduit à $\overline{\phi_{z}^{+}}$. En particulier, on en déduit que $\omega\left(\phi_{z_{n}}^{+}\right)=z^{*}$ pour $n$ assez grand.

On déduit de ce qui précède que $\omega\left(\phi_{z_{l}^{-}}\right)=\omega\left(\phi_{z_{l}^{+}}\right)$. On définit de même, pour $l$ impair des points $z_{l}^{-}$et $z_{l}^{+}$de $\breve{\gamma}_{l}$ avec $\alpha\left(\phi_{z_{l}^{-}}\right)=\alpha\left(\phi_{z_{l}^{+}}\right)$. Par un raisonnement du même type, on montre que pour tout $l$ pair, on a

$$
\omega\left(\phi_{z_{l}^{+}}\right)=\alpha\left(\phi_{z_{l+1}^{-}}\right) .
$$


Pour cela, on choisit une suite $\left(z_{n}\right) \in \mathrm{I}_{l}$, où $l^{\prime}\left(z_{n}\right)=l+1$, convergeant vers $z_{l}^{+}$. Le segment $\phi_{z_{n}} \cap \overline{\mathrm{U}}$ joint $z_{n}$ à un point $z_{n}^{\prime} \in \mathrm{I}_{l+1}$ et la suite $\left(z_{n}^{\prime}\right)_{n \geq 0}$ converge vers $z_{l+1}^{-}$. On choisit une valeur d'adhérence $\mathrm{K}$ de la suite $\phi_{z_{n}} \cap \overline{\mathrm{U}}$, on sait que $\mathrm{K}$ est formé de $\overline{\phi_{z_{l}^{+}}^{+}}, \overline{\phi_{z_{l+1}^{-}}^{-}}$et de points de $\mathrm{X}$. Comme $\mathrm{K}$ est connexe, c'est la réunion des deux premiers ensembles et on a $\omega\left(\phi_{z_{l}^{+}}\right)=\alpha\left(\phi_{z_{l+1}^{-}}\right)$. On obtient de même $\omega\left(\phi_{z_{l}^{-}}\right)=\alpha\left(\phi_{z_{l-1}^{+}}\right)$. Ainsi, de proche en proche, par le même raisonnement, on montre que tous les points $\omega\left(\phi_{z_{l}^{ \pm}}\right)$pour $l$ pair et $\alpha\left(\phi_{z_{l}^{ \pm}}\right)$pour $l$ impair coïncident. On remarque enfin que la réunion des traces des feuilles dans $\bar{U}$ et du point limite commun $z_{*}$ est fermée, il ne peut pas y avoir d'autre point dans $\overline{\mathrm{U}}$. Ainsi, le feuilletage sur $\overline{\mathrm{U}}$ est un feuilletage de type selle. Il y a un nombre fini de modèles possibles à $q$ fixé.

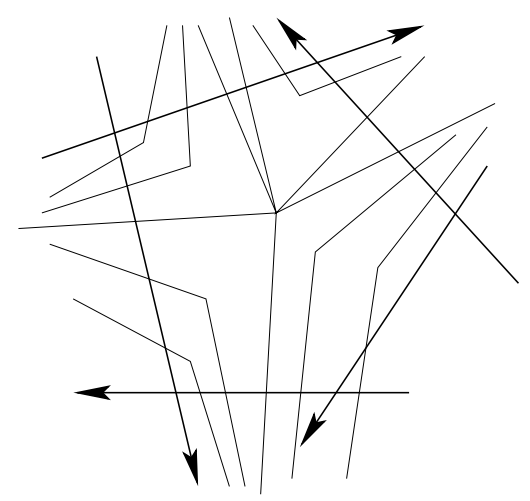

Dans le cas où $q=1$ et $\mathrm{X} \cap \mathrm{U} \neq \emptyset$, on a une situation analogue. Si par contre, $\mathrm{X} \cap \mathrm{U}=\emptyset$, alors $\overline{\mathrm{U}}$ est feuilleté par les traces des feuilles de $\mathscr{F}$.
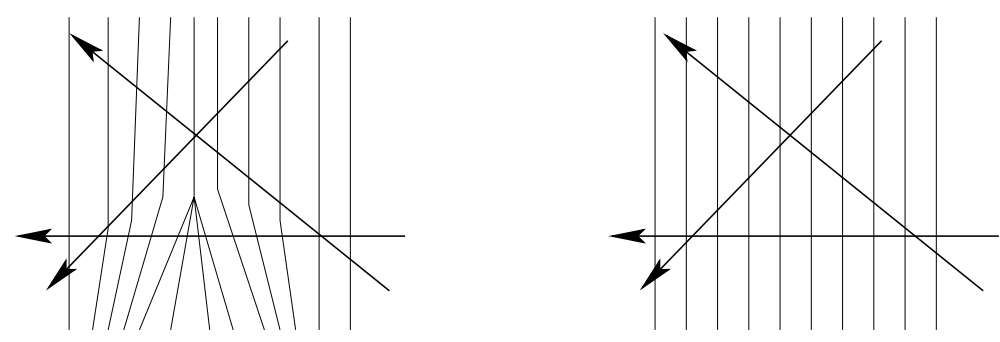

Si $\mathrm{U}$ est une composante connexe telle que $\partial \mathrm{U}=\partial^{+} \mathrm{U}$ (correspondant donc à un minimum local de $\Lambda$ ), on démontre de la même façon que $\overline{\mathrm{U}}$ est formé des demi-droites $\phi_{z}^{+}, z \in \partial \mathrm{U}$, et du point limite commun $z^{*}=\omega\left(\phi_{z}^{+}\right)$. Le feuilletage est radial. On a un résultat analogue pour une composante correspondant à un maximum local de $\Lambda$. 
Preuve de iv). - Fixons $z_{0} \in \mathrm{X}$ et notons $\mathrm{U}$ la composante connexe de $\mathrm{M} \backslash \Gamma$ qui contient $z_{0}$. Soit $z$ un point suffisamment proche de $z_{0}$ pour que l'arc $\gamma_{z}: t \mapsto \mathrm{F}_{t}^{\prime}(z)$ prenne ses valeurs dans U. Nous allons construire un arc à valeurs dans $\mathrm{U}$, joignant $z$ à $\mathrm{F}(z)$ et positivement transverse à $\mathscr{F}$. Nous savons qu'il existe un arc $\gamma$ joignant $z$ à $\mathrm{F}(z)$, défini sur $[0,1]$, positivement transverse à $\mathscr{F}$ et homotope à $\gamma_{z}$ dans $\mathrm{M} \backslash \mathrm{X}$. Remarquons d'abord que toute feuille $\phi_{\gamma(t)}$ rencontre $\mathrm{U}$. C'est évident si la feuille contient $z$ ou $\mathrm{F}(z)$. Si elle ne contient aucun de ces points, le nombre d'intersection $\gamma \wedge \phi_{\gamma(t)}$ est bien défini (puisque les feuilles définissent des connexions) et il est strictement positif. De plus, on a $\gamma \wedge \phi_{\gamma(t)}=\gamma_{z} \wedge \phi_{\gamma(t)}$. Puisque $\gamma_{z}$ prend ses valeurs dans U, la feuille $\phi_{\gamma(t)}$ rencontre U. L'ensemble des points $t \in[0,1]$ tels que $\gamma(t) \in \mathrm{U}$ est ouvert dans [0,1]. Son complémentaire est formé de deux parties fermées disjointes, les ensembles

$$
\left\{t \in[0,1] \mid \phi_{\gamma(t)} \cap \mathrm{U} \subset \phi_{\gamma(t)}^{-}\right\}, \quad\left\{t \in[0,1] \mid \phi_{\gamma(t)} \cap \mathrm{U} \subset \phi_{\gamma(t)}^{+}\right\} .
$$

On peut donc, par holonomie du feuilletage, construire un arc $\gamma^{\prime}$ joignant $z$ à $\mathrm{F}(z)$, positivement transverse à $\mathscr{F}$ et à valeurs dans $\mathrm{U}$.

Si $z$ est un point selle, on vérifie aisément que

$$
\gamma \wedge \phi_{\gamma(t)}=\gamma^{\prime} \wedge \phi_{\gamma(t)}=1
$$

si $t \in$ ]0, 1[, et qu'aucune des feuilles $\phi_{\gamma(t)}$ ne passent par $z$ ou par $\mathrm{F}(z)$. On montre ensuite que $i\left(\mathrm{~F}, z_{0}\right)=i\left(\mathscr{F}, z_{0}\right)$ ce qui prouve l'assertion iv). En effet, notons $\mathscr{F}_{\mathrm{U}}$ le feuilletage restreint à U. Si $\Gamma^{\prime}$ est le bord d'un petit disque contenant $z_{0}$ dans son intérieur, et si $\Phi: \Gamma^{\prime} \rightarrow \mathrm{U}$ est une fonction continue telle que, pour tout $z \in \Gamma^{\prime}$, le point $\Phi(z)$ appartient à la demi-feuille positive ouverte $\phi_{z}^{+} \cap \mathrm{U} \backslash\{z\}$ de $\mathscr{F}_{\mathrm{U}}$ issue de $z$, on peut trouver une homotopie $\left(\mathrm{F}^{s}\right)_{s \in[0,1]}$ entre $\Phi$ et $\left.\mathrm{F}\right|_{\Gamma^{\prime}}$, telle qu'aucune application $\mathrm{F}^{s}: \Gamma^{\prime} \rightarrow \mathrm{U}$ n'a de point fixe, ce qui implique l'égalité $i\left(\mathrm{~F}, z_{0}\right)=i\left(\mathscr{F}, z_{0}\right)$. Pour cela, il suffit de remarquer qu'on peut construire un fibré sur $\Gamma^{\prime}$ à fibre contractile (homéomorphe à $\left.\left.\{0\} \times\right] 0,+\infty[\cup] 0,1\right] \times \mathbf{R}$ ) dont la fibre $\mathrm{O}_{z}$ est la réunion des feuilles de $\mathscr{F}_{\mathrm{U}}$, autres que $\phi_{z} \cap \mathrm{U}$, qui sont rencontrées par un arc $\gamma:[0,1] \rightarrow \mathrm{U}$ positivement transverse joignant $z$ à $\mathrm{F}(z)$ et de la demifeuille ouverte $\phi_{z}^{+} \cap U \backslash\{z\}$. L'ensemble des sections continues est alors connexe par arcs et chaque section est en fait une application continue de $\Gamma^{\prime}$ dans $\mathrm{U}$ sans point fixe. Parmi ces sections, on trouve $\Phi$ et $\left.\mathrm{F}\right|_{\Gamma^{\prime}}$.

Par contre, si $z_{0}$ est un puits ou une source, le feuilletage est radial sur U, l'entier $\gamma \wedge \phi_{\gamma(t)}$ peut être arbitrairement grand, la structure du feuilletage ne donne aucune information sur l'indice de Lefschetz au point $z_{0}$. Cependant, puisque $\mathrm{F}$ préserve une mesure chargeant les ouverts, on sait d'après un théorème de PelikanSlaminka [PS] (voir [LeC3], voir également [N] ou [Sim] pour le cas des difféomorphismes) que l'indice de Lefschetz de tout point fixe de $\mathrm{F}$ est inférieur ou égal 
à 1. Utilisant la formule de Poincaré-Hopf, on en déduit :

$$
\sum_{z \in \mathrm{X}} i(\mathrm{~F}, z) \leq \sum_{z \in \mathrm{X}} i(\mathscr{F}, z)=2-2 g .
$$

Preuve de v). - Un cas particulier de la formule de Lefschetz nous dit que si F est un homéomorphisme isotope à l'identité d'une surface compacte de genre $g$, et qui a un nombre fini de points fixes, alors

$$
\sum_{\mathrm{F}(z)=z} i(\mathrm{~F}, z)=2-2 g .
$$

La formule de Nielsen (voir Jiang [J]) permet d'être plus précis : si $\mathrm{F}$ a un nombre fini de points fixes contractiles, alors

$$
\sum_{z \in \mathrm{Fix}_{*}(\mathrm{~F})} i(\mathrm{~F}, z)=2-2 g .
$$

Ainsi dans le cas, où $\mathrm{X}=\mathrm{Fix}_{*}(\mathrm{~F})$, on obtient :

$$
2-2 g=\sum_{z \in \mathrm{X}} i(\mathrm{~F}, z) \leq \sum_{z \in \mathrm{X}} i(\mathscr{F}, z)=2-2 g,
$$

ce qui n'est possible que si l'indice de Lefschetz d'un puits ou d'une source de $\mathscr{F}$ est égal à 1 .

On va finir cette section en liant la fonction $\Lambda$ que l'on vient de définir et la classique fonction d'action $\mathrm{L}$ dans le cas où $\mathrm{F}$ est un difféomorphisme. On supposera par commodité que $\mathrm{X}=\mathrm{Fix}_{*}(\mathrm{~F})$ est non enlacé. La surface $\mathrm{N}=$ $\mathrm{M} \backslash \mathrm{Fix}_{*}(\mathrm{~F})$ se compactifie naturellement en une variété à bord $\overline{\mathrm{N}}$ en rajoutant, pour chaque point $z \in \mathrm{Fix}_{*}(\mathrm{~F})$, le cercle $\Sigma_{z}$ des demi-droites du plan tangent $\mathrm{T}_{z} \mathrm{M}$. Le difféomorphisme $F_{\mid N}$ se prolonge alors en un homéomorphisme $\overline{\mathrm{F}}$ de $\overline{\mathrm{N}}$, isotope à l'identité, dont la restriction à $\Sigma_{z}$ est donnée par l'action naturelle de l'application linéaire tangente $\mathrm{DF}(z)$. Si la mesure $\mu$ invariante par $\mathrm{F}$ ne charge aucun des points $z \in \mathrm{Fix}_{*}(\mathrm{~F})$, elle définit sur $\overline{\mathrm{N}}$ une mesure invariante par $\overline{\mathrm{F}}$, notée également $\mu$. On peut donc définir son vecteur de rotation dans $\mathrm{H}_{1}(\overline{\mathrm{N}}, \mathbf{R})$. L'inclusion

$$
\iota: \mathrm{N} \hookrightarrow \overline{\mathrm{N}}
$$

induit un isomorphisme

$$
\iota_{*}: \mathrm{H}_{1}(\mathrm{~N}, \mathbf{R}) \rightarrow \mathrm{H}_{1}(\overline{\mathrm{N}}, \mathbf{R}),
$$


et on notera $\rho_{\mathrm{N}}(\mu) \in \mathrm{H}_{1}(\mathrm{~N}, \mathbf{R})$ le vecteur de rotation défini via cet isomorphisme. Si $\Gamma$ est un arc à valeurs dans $\mathrm{N}$, qui joint $z_{0} \in \mathrm{Fix}_{*}(\mathrm{~F})$ à $z_{1} \in \mathrm{Fix}_{*}(\mathrm{~F})$, on peut définir le nombre d'intersection $\Gamma \wedge \kappa$ pour toute classe $\kappa \in \mathrm{H}_{1}(\mathrm{~N}, \mathbf{R})$, en particulier on peut définir $\Gamma \wedge \rho_{\mathrm{N}}(\mu)$ et même $\Gamma \wedge \rho_{\mathrm{N}}(z)$ si $z$ a un nombre de rotation dans $\mathrm{N}$. Remarquons que $\Gamma \wedge \rho_{\mathrm{N}}(\mu)$ est indépendant de $\Gamma$ (contrairement à $\left.\Gamma \wedge \rho_{\mathrm{N}}(z)\right)$, car le vecteur de rotation $\rho(\mu) \in \mathrm{H}_{1}(\mathbf{M}, \mathbf{R})$ est nul. De plus, on a

$$
\Gamma \wedge \rho_{\mathrm{N}}(\mu)=\mathrm{L}\left(z_{1}\right)-\mathrm{L}\left(z_{0}\right),
$$

où

$$
\mathrm{L}: \mathrm{Fix}_{*}(\mathrm{~F}) \rightarrow \mathbf{R}
$$

est définie à une constante près. On dira que L est la fonction d'action.

Proposition 10.5. - On suppose que $\mathrm{F}$ est un difféomorphisme, que $\mathrm{Fix}_{*}(\mathrm{~F})=\mathrm{X}$ est fini et que la mesure ne charge aucun point de $\operatorname{Fix}_{*}(\mathrm{~F})$.

i) S'il existe dans $\mathscr{F}$ une connexion de $z_{0}$ à $z_{1}$, alors $\mathrm{L}\left(z_{1}\right)<\mathrm{L}\left(z_{0}\right)$.

ii) Pour tout $\varepsilon>0$, on peut choisir les lacets $\Gamma_{i}$ et les entiers $m_{i}$ défnissant la fonction $\Lambda$, puis un entier $q>0$, tels que

$$
\left|\frac{1}{q} \Lambda(z)-\mathrm{L}(z)\right|<\varepsilon \text {, pour tout } z \in \mathrm{X} \text {. }
$$

Démonstration. - Montrons i). On suppose que $\phi$ est une feuille qui joint $z_{0} \in \mathrm{X}$ à $z_{1} \in \mathrm{X}$. On note $\rho_{\mathrm{N}}(z) \in \mathrm{H}_{1}(\mathrm{~N}, \mathbf{R})$ le nombre de rotation d'un point $z \in \mathrm{N}$ et $\rho_{\mathrm{N}}(\mu) \in \mathrm{H}_{1}(\mathrm{~N}, \mathbf{R})$ celui de la mesure $\mu$. Il faut prouver que $\phi \wedge \rho_{\mathrm{N}}(\mu)<0$.

L'assertion ii) de la proposition 8.2 nous dit que $\phi \wedge \rho_{\mathrm{N}}(z) \leq 0$ pour tout point récurrent $z \in \mathrm{N}$ admettant un vecteur de rotation dans $\mathrm{N}$. Puisque

$$
\phi \wedge \rho_{\mathrm{N}}(\mu)=\int_{\mathrm{N}} \phi \wedge \rho_{\mathrm{N}}(z) d \mu
$$

il suffit de trouver un ensemble de mesure non nulle où la fonction $z \mapsto \phi \wedge \rho_{\mathrm{N}}(z)$ est strictement négative. Fixons $z_{2} \in \phi$ ainsi qu'un disque $U$ contenant $z_{2}$, où le feuilletage est trivial. Pour tout couple $\left(z, z^{\prime}\right) \in \mathrm{U}^{2}$, choisissons un arc $\gamma_{z, z^{\prime}}$ à valeurs dans $\mathrm{U}$, qui joint $z$ à $z^{\prime}$. Notons $\Phi: z \mapsto \mathrm{F}^{\tau(z)}(z)$ l'application de premier retour définie sur $\mathrm{R} \cap \mathrm{U}$, où $\mathrm{R}$ est l'ensemble des points récurrents de $\mathrm{F}$, définissons le lacet

$$
\Gamma_{z}=\left(\prod_{i=0}^{\tau(z)-1} \gamma_{\mathrm{F}^{i}(z)}\right) \gamma_{\Phi(z), z},
$$


et notons $\left[\Gamma_{z}\right]_{\mathrm{N}} \in \mathrm{H}_{1}(\mathrm{~N}, \mathbf{Z})$ sa classe d'homologie. On vérifie aisément que $\phi \wedge \Gamma_{z}$ $\leq 0$, pour tout $z \in \mathrm{U}$, et que l'inégalité est stricte si $z$ est proche de $z_{2}$. On déduit du théorème ergodique de Birkhoff et de l'égalité

$$
\rho_{\mathrm{N}}(z)=\lim _{n \rightarrow+\infty}\left(\sum_{i=0}^{n-1} v\left(\Phi^{i}(z)\right)\right)^{-1}\left(\sum_{i=0}^{n-1}\left[\Gamma_{\Phi^{i}(z)}\right]_{\mathrm{N}}\right),
$$

pour tout $z \in \mathrm{U}$, que $\phi \wedge \rho_{\mathrm{N}}(z)<0$ pour un ensemble non négligeable de points de U.

Pour montrer ii), munissons $\mathrm{H}_{1}(\mathrm{M}, \mathbf{R})$ d'une norme \|\|$_{\mathrm{M}}$ et $\mathrm{H}_{1}(\mathrm{~N}, \mathbf{R})$ d'une norme \|\|$_{\mathrm{N}}$. Pour tout couple $\left(z, z^{\prime}\right)$ de points de $\mathrm{X}$, choisissons un $\operatorname{arc} \Gamma_{z, z^{\prime}}$ à valeurs dans $\mathrm{N}$ joignant $z$ à $z^{\prime}$. Il existe $\eta>0$ tel que pour tout $\kappa \in \mathrm{H}_{1}(\mathrm{~N}, \mathbf{R})$ et pour tout $\left(z, z^{\prime}\right) \in \mathrm{X}^{2}$ :

$$
\|\kappa\|_{\mathrm{N}}<\eta \Longrightarrow\left|\Gamma_{z, z^{\prime}} \wedge \kappa\right|<\varepsilon
$$

Tout élément $\kappa \in \mathrm{H}_{1}(\mathrm{M}, \mathbf{Q})$ proche de 0 s'écrit $\kappa=\sum_{i=0}^{n} \beta_{i}\left[\Gamma_{i}\right]_{\mathrm{M}}$, où chaque $\beta_{i}$ est petit, rationnel et strictement positif. En particulier, il existe $\delta>0$, tel que

$$
\|\kappa\|_{\mathrm{M}}<\delta \Longrightarrow\left\|\sum_{i=0}^{n} \beta_{i}\left[\Gamma_{i}\right]_{\mathrm{N}}\right\|_{\mathrm{N}}<\frac{\eta}{2}
$$

On peut approximer la mesure $\mu$ par un barycentre à coefficients strictement positifs $\sum_{j=0}^{l} \alpha_{j} \mu_{j}$ de mesures de probabilité ergodiques invariantes $\mu_{j}$. Pour $\mu_{j}$ presque tout point $z \in \mathrm{N}$, on a

$$
\rho_{\mathrm{M}}(z)=\rho_{\mathrm{M}}\left(\mu_{j}\right), \quad \rho_{\mathrm{N}}(z)=\rho_{\mathrm{N}}\left(\mu_{j}\right) .
$$

Rappelons que $\rho_{\mathrm{M}}(\mu)=0$. On peut donc trouver une suite $\left(z_{j}\right)_{0 \leq i \leq l}$ de points positivement récurrents ayant un vecteur de rotation dans $\mathrm{M}$ et dans $\mathrm{N}$ tels que

$$
\left\|\rho_{\mathrm{N}}(\mu)-\sum_{j=0}^{l} \alpha_{j} \rho_{\mathrm{N}}\left(z_{j}\right)\right\|_{\mathrm{N}}<\frac{\eta}{2}, \quad\left\|\sum_{j=0}^{l} \alpha_{j} \rho_{\mathrm{M}}\left(z_{j}\right)\right\|_{\mathrm{M}}<\delta .
$$

En utilisant la proposition 8.2 on peut donc trouver une famille $\left(\Gamma_{j}^{\prime}\right)_{0 \leq i \leq l}$ de lacets dans $\mathrm{N}$ transverses à $\mathscr{F}$ ainsi qu'une famille $\left(\beta_{j}^{\prime}\right)_{0 \leq j \leq l}$ de nombres rationnels strictement positifs, telles que

$$
\left\|\rho_{\mathrm{N}}(\mu)-\sum_{j=0}^{l} \beta_{j}^{\prime}\left[\Gamma_{j}^{\prime}\right]_{\mathrm{N}}\right\|_{\mathrm{N}}<\frac{\eta}{2}, \quad\left\|\sum_{j=0}^{l} \beta_{j}^{\prime}\left[\Gamma_{j}^{\prime}\right]_{\mathrm{M}}\right\|_{\mathrm{M}}<\delta .
$$


Par définition de $\delta$ et en utilisant l'inégalité triangulaire, on peut alors trouver une famille $\left(\beta_{i}\right)_{0 \leq i \leq n}$ de nombres rationnels strictement positifs telle que

$$
\sum_{j=0}^{l} \beta_{j}^{\prime}\left[\Gamma_{j}^{\prime}\right]_{\mathrm{M}}+\sum_{i=0}^{n} \beta_{i}\left[\Gamma_{i}\right]_{\mathrm{M}}=0
$$

et

$$
\left\|\rho_{\mathrm{N}}(\mu)-\sum_{j=0}^{l} \beta_{j}^{\prime}\left[\Gamma_{j}^{\prime}\right]_{\mathrm{N}}-\sum_{i=0}^{n} \beta_{i}\left[\Gamma_{i}\right]_{\mathrm{N}}\right\|_{\mathrm{N}}<\eta .
$$

Ajoutons à notre famille $\left(\Gamma_{i}\right)_{0 \leq i \leq n}$ la famille $\left(\Gamma_{j}^{\prime}\right)_{0 \leq j \leq m}$ et écrivons

$$
\beta_{j}^{\prime}=\frac{m_{j}^{\prime}}{q}, \quad \beta_{i}=\frac{m_{i}}{q},
$$

où les entiers $m_{j}^{\prime}, m_{i}$, ainsi que l'entier $q$ sont strictement positifs. Si $\Lambda$ est la fonction associée au lacet

$$
\prod_{i=0}^{l} \Gamma_{j}^{\prime m_{j}^{\prime}} \prod_{i=0}^{n} \Gamma_{i}^{m_{i}}
$$

alors $\frac{1}{q} \Lambda$ est proche de $\mathrm{L}$ sur $\mathrm{X}$, à $\varepsilon$ près.

Remarquons que nous avons démontré :

Corollaire 10.6. - Si $\mathrm{F}$ vérifie les hypothèses du théorème 10.1 et si $\mathrm{Fix}_{*}(\mathrm{~F})$ est non enlacé, alors l'indice de Lefschetz d'un point $z \in \mathrm{Fix}_{*}(\mathrm{~F})$ où l'action est minimale ou maximale est égal à 1 .

Démonstration. - Il suffit de remarquer qu'un point $z$ où l'action est minimale (resp. maximale) est nécessairement un puits (resp. une source) du feuilletage $\mathscr{F}$ et que $i(\mathrm{~F}, z)=i(\mathscr{F}, z)=1$.

\section{Existence de points périodiques pour des homéomorphismes hamiltoniens de surfaces}

Nous montrerons dans cette section l'assertion ii) du théorème 1.5 énoncé en section 1 :

Théorème 11.1. - Soit $\mathrm{M}$ une surface compacte de genre $g \geq 1$ et $\left(\mathrm{F}_{t}\right)_{t \in[0,1]}$ une isotopie dans $\mathrm{M}$ joignant l'identité à un homéomorphisme $\mathrm{F}$. On suppose que $\mathrm{F}$ préserve une mesure borélienne de probabilité dont le support est $\mathrm{M}$ et que le nombre de rotation de cette 
mesure est nul. S'il n'existe qu'un nombre fini de point fixes contractiles, il existe des points périodiques contractiles de périodes arbitrairement grandes.

On a un résultat analogue pour une surface à bord $\mathrm{M}$ autre que le disque fermé. En effet l'homéomorphisme défini naturellement par réflexion sur le double de $\mathrm{M}$ vérifie les hypothèses du théorème 11.1. On déduit également de ce théorème :

Corollaire 11.2. - Soit $(\mathrm{M}, \omega)$ une surface compacte de genre $g \geq 1$ munie d'une forme symplectique et $\left(\mathrm{F}_{t}\right)_{t \in \mathbf{R}}$ une isotopie hamiltonienne. Alors $\mathrm{F}_{1}$ possède une infinité de points périodiques contractiles.

Nous supposerons dans la suite de la section que $g \geq 2$. Le cas $g=1$ se traite de façon analogue en remplaçant le disque de Poincaré $\mathbf{D}=\{z \in \mathbf{C} \mid$ $|z|<1\}$ par le plan complexe et en faisant attention à bien fixer l'isotopie. Notons $\pi: \mathbf{D} \rightarrow \mathrm{M}$ le revêtement universel de $\mathrm{M}$ et $\mathrm{G}$ le groupe des transformations de recouvrement. L'isotopie $\left(\mathrm{F}_{t}\right)_{t \in[0,1]}$ se relève en une isotopie $\left(f_{t}\right)_{t \in[0,1]}$ issue de l'identité et aboutissant à un homéomorphisme $f$, qui est indépendant de l'isotopie, c'est le relèvement canonique de $\mathbf{F}$. Nous savons, c'est un résultat classique, que $f$ se prolonge en un homéomorphisme du même nom sur $\overline{\mathbf{D}}$ laissant fixe tout point de $\mathrm{S}^{1}=\{z \in \mathbf{G}|| z \mid=1\}$ (voir Handel et Thurston [HT]). Un point fixe $z$ de $\mathrm{F}$ est contractile si tout antécédent de $z$ par $\pi$ est fixe par $f$.

Nous utiliserons le corollaire 9.2 dans la preuve. Il est facile en effet, sous les hypothèses du théorème 11.1, de construire des homéomorphismes de l'anneau vérifiant la propriété d'intersection. Expliquons comment. Pour tout point fixe $z$ de $f$, considérons l'anneau $\mathbf{A}_{z}=\overline{\mathbf{D}} \backslash\{z\}$. Puisque $f$ préserve une mesure localement finie, la restriction $f_{z}$ de $f$ à $\mathrm{A}_{z}$ vérifie la propriété d'intersection. De même si $z^{\prime}$ est un autre point fixe, on construit un anneau $\mathrm{A}_{z, z^{\prime}}$ en identifiant dans $\overline{\mathbf{D}} \backslash\left\{z, z^{\prime}\right\}$ le cercle $\mathrm{S}^{1}$ à un point $\infty$. La restriction de $f$ à $\overline{\mathbf{D}} \backslash\left\{z, z^{\prime}\right\}$ définit naturellement un homéomorphisme $f_{z, z^{\prime}}$ de $\mathrm{A}_{z, z^{\prime}}$ qui fixe $\infty$ et qui vérifie la propriété d'intersection. Pour définir les vecteurs de rotation, on choisira, dans le cas de $\mathrm{A}_{z}$, le relèvement $\widehat{f}_{z}$ de $f_{z}$ au revêtement universel qui fixe tous les points du revêtement de $\mathrm{S}^{1}$; dans le cas de $\mathrm{A}_{z, z^{\prime}}$, le relèvement $\widehat{f}_{z, z^{\prime}}$ qui fixe les antécédents de $\infty$.

Le résultat fondamental de cette section est le suivant :

Proposition 11.3. - Il existe deux points fixes distincts $z$ et $z^{\prime}$ de $f$ et un point $z^{\prime \prime} \in \mathrm{A}_{z, z^{\prime}}$ ayant un vecteur de rotation non nul, pour le relèvement $\widehat{f}_{z, z^{\prime}}$.

Le théorème 11.1 se déduit immédiatement de la proposition 11.3. En effet, puisque le nombre de rotation de $\infty$ est nul, on déduit du corollaire 9.2 que $f$ possède des orbites périodiques de périodes arbitrairement grandes. Il reste à re- 
marquer que si $z$ est un point périodique de $f$ de période $q$, alors $\pi(z)$ est un point périodique de $\mathrm{F}$ de même période. Sinon, on trouverait $q^{\prime}<q$ et $\mathrm{T} \in \mathrm{G} \backslash\left\{\operatorname{Id}_{\mathbf{D}}\right\}$ tel que $f^{q^{\prime}}(z)=\mathrm{T}(z)$. On en déduirait $\mathrm{T}^{q}(z)=f^{q q^{\prime}}(z)=z$, ce qui est impossible puisque $\mathrm{T}$ n'a pas d'orbite périodique.

Il existe un cas simple où la proposition 11.3 est vérifiée. Supposons que F soit de classe $\mathrm{C}^{1}$. Pour tout point fixe $z$ de $f$, l'anneau $\mathbf{A}_{z}$ se compactifie naturellement en un anneau $\overline{\mathrm{A}}_{z}=\Sigma_{z} \sqcup \mathrm{A}$, si on rajoute le cercle des demi-droites réelles issues de $z$. Puisque $f$ est différentiable en $z$, on peut prolonger $f_{z}$ en un homéomorphisme $\bar{f}_{z}$ de $\overline{\mathrm{A}}_{z}$ dont la restriction à $\Sigma_{z}$ est donnée par l'action naturelle de la différentielle $\mathrm{D} f(z)$ sur les demi-droites issues de $z$. La classe d'homologie du lacet $\Gamma_{1}: t \mapsto e^{2 i \pi t}$, défini sur $[0,1]$, engendre $\mathrm{H}_{1}\left(\overline{\mathrm{A}}_{z}, \mathbf{R}\right)$, et permet de définir le nombre de rotation $\theta(z)$ de $\Sigma_{z}$ appelé communément le nombre de torsion de $z$. Si $z^{\prime}$ est un autre point fixe de $f$, on peut compactifier $\mathrm{A}_{z, z^{\prime}}$ en rajoutant les cercles $\Sigma_{z}$ et $\Sigma_{z^{\prime}}$. On peut définir les nombres de rotation sur $\overline{\mathrm{A}}_{z, z^{\prime}}$ en choisissant comme générateur de $\mathrm{H}_{1}\left(\overline{\mathrm{A}}_{z, z^{\prime}}\right)$ la classe du lacet $\Gamma: t \mapsto z+\varepsilon e^{2 i \pi t}$, où $\varepsilon$ est assez petit. Les nombres de rotation induits sur les cercles $\Sigma_{z}$ et $\Sigma_{z^{\prime}}$ sont alors respectivement $\theta(z)$ et $-\theta\left(z^{\prime}\right)$.

Ainsi, s'il existe un point fixe contractile $z$ de nombre de torsion non nul, la proposition 11.3 est vérifiée au compactifié de $A_{z, T z}$, où $T \in G^{*}$, et on peut en déduire le théorème 11.1. Un cas important où cette propriété est vérifiée est le cas générique où pour tout point fixe contractile $z$, l'application linéaire tangente $\operatorname{DF}(z)$ n'admet pas 1 comme valeur propre (dans le cas des surfaces c'est la condition générique vérifiée dans le théorème de Salamon-Zehnder [SZ]). On peut montrer qu'il existe un point fixe contractile $z$ dont l'indice de Lefschetz est 1 . On en déduit que les valeurs propres de $\operatorname{DF}(z)$ sont complexes conjuguées ou réelles négatives et donc que le nombre de torsion de $z$ n'est pas entier. Ainsi la proposition 11.3, qui donne l'existence d'un anneau $\mathrm{A}_{z, z^{\prime}}$ où il $\mathrm{y}$ a de la torsion, exprime qu'une condition de généricité faible est toujours vérifiée dès que l'ensemble des points fixes contractiles est fini.

Nous allons commencer par deux cas simples où la propriété de torsion se montre facilement:

Lemme 11.4. - La proposition 11.3 est vérifiée dans les cas suivants :

i) la restriction de $\mathrm{F}$ à $\mathrm{M} \backslash \mathrm{Fix}_{*}(\mathrm{~F})$ n'est pas isotope à l'identité (ce qui signifie que l'ensemble $\operatorname{Fix}_{*}(\mathrm{~F})$ est enlacé) ;

ii) il existe un point périodique contractile qui n'est pas fixe.

Dans le premier cas, $i l$ existe trois points fixes distincts $z_{0}, z_{1}$ et $z_{2}$ du relèvement canonique $f$ de $\mathrm{F}$ à $\mathbf{D}$ tel que le vecteur de rotation de $z_{0}$ dans l'anneau $\mathrm{A}_{z_{1}, z_{2}}$ pour le relèvement $\widehat{f}_{z_{1}, z_{2}}$ est non nul. Dans le second cas, si zo est un point périodique de $f$ qui n'est pas fixe, il existe 
deux points fixes distincts $z_{1}$ et $z_{2}$ de $f$ tel que le vecteur de rotation de l'orbite de $z_{0}$ pour le relèvement $\widehat{f}_{z_{1}, z_{2}}$ est également non nul.

Démonstration. - Commençons par démontrer i). On considère une partie non enlacée maximale $\mathrm{X} \subset \mathrm{Fix}_{*}(\mathrm{~F})$ et une $\underset{\sim}{\mathrm{X}}$ isopie $\left(\mathrm{F}_{t}^{\prime}\right)_{t \in[0,1]}$ de l'identité à $\mathrm{F}$ qui laisse fixe tout point de $\mathrm{X}$. On pose alors $\tilde{\mathrm{X}}=\pi^{-1}(\mathrm{X}) \subset \mathbf{D}$ et on note $\left(f_{t}^{\prime}\right)_{t \in[0,1]}$ l'isotopie relevée, issue de l'identité. On considère un feuilletage $\mathscr{F}$ sur $\mathrm{M} \backslash \mathrm{X}$ transverse à l'isotopie $\left(\mathrm{F}_{t}^{\prime}\right)_{t \in[0,1]}$ et on note $\widetilde{\mathscr{F}}$ le feuilletage relevé défini sur $\mathbf{D} \backslash \widetilde{\mathrm{X}}$. Puisque $\mathrm{X} \neq \mathrm{Fix}_{*}(\mathrm{~F})$, on peut choisir $z_{0} \in \operatorname{Fix}(f) \backslash \tilde{\mathrm{X}}$. On considère alors le lacet $\Gamma_{0}=\gamma_{z_{0}}: t \mapsto f_{t}^{\prime}\left(z_{0}\right)$ ainsi qu'un lacet $\Gamma_{1}$ basé en $z_{0}$ positivement transverse à $\widetilde{\mathscr{F}}$ et homotope dans $\mathbf{D} \backslash \widetilde{\mathrm{X}}$ à $\Gamma_{0}$.

Pour tout lacet $\Gamma$ (défini sur $[0,1]$ ) à valeurs dans $\mathbf{D}$, et pour tout point $z \notin \Gamma$, il existe une unique application continue $\xi: \mathrm{S}^{1} \rightarrow \mathrm{S}^{1}$ telle que

$$
\xi\left(e^{2 i \pi t}\right)=\frac{\Gamma(t)-z}{|\Gamma(t)-z|} .
$$

Le degré de cet application est l'indice $\operatorname{Ind}_{\Gamma}(z)$ de $\Gamma$ par rapport à $z$. Si $\Gamma^{\prime}$ est un arc issu de $z$, défini sur $\left[0,+\infty\left[\right.\right.$, et vérifiant $\lim _{t \rightarrow \infty}\left|\Gamma^{\prime}(t)\right|=1$, alors $\operatorname{Ind}_{\Gamma}(z)$ est le nombre d'intersection algébrique $\Gamma^{\prime} \wedge \Gamma$.

Les fonctions

$$
\lambda_{0}=\operatorname{Ind}_{\Gamma_{0}}: \mathbf{D} \backslash \Gamma_{0} \rightarrow \mathbf{Z}, \quad \lambda_{1}=\operatorname{Ind}_{\Gamma_{1}}: \mathbf{D} \backslash \Gamma_{1} \rightarrow \mathbf{Z}
$$

coïncident sur $\tilde{\mathrm{X}}$ puisque $\Gamma_{0}$ et $\Gamma_{1}$ sont homotopes sur $\mathbf{D} \backslash \widetilde{\mathrm{X}}$. On peut toujours supposer, quitte à perturber légèrement $\Gamma_{1}$, que $\Gamma_{1}$ n'a pas de point triple ou d'ordre supérieur et qu'il a un nombre fini de points doubles avec des autointersections transverses. La fonction $\lambda_{1}$ n'est pas constante et on a donc $\max \lambda_{1}>0$ ou $\min \lambda_{1}<0$. Plaçons nous dans le premier cas. Puisque $\lambda_{1}$ est à support compact, toute composante connexe $U$ de $\mathbf{D} \backslash \Gamma_{1}$, où $\lambda_{1}$ atteint son maximum, est un disque ouvert et sa frontière est une courbe fermée simple transverse au feuilletage. Cette composante contient donc un point $z_{1} \in \widetilde{\mathrm{X}}$. La fonction $\lambda_{1}$ s'annule en tout point fixe $z_{2}$ suffisamment proche du cercle unité. Il reste à remarquer que le nombre de rotation du point fixe $z_{0}$ dans l'anneau $\mathrm{A}_{z_{1}, z_{2}}$ est égal à

$$
\lambda_{0}\left(z_{1}\right)-\lambda_{0}\left(z_{2}\right)=\lambda_{1}\left(z_{1}\right)
$$

il est donc strictement positif.

Démontrons maintenant ii). Si $\mathrm{F}$ a un point périodique contractile de période $q>1$, alors $f$ admet un point périodique $z_{0}$ de même période. On reprend le même raisonnement, où $\mathrm{X}$ peut cette fois être égal à $\mathrm{Fix}_{*}(\mathrm{~F})$. On construit un lacet $\Gamma_{1}$ basé en $z_{0}$, positivement transverse à $\widetilde{\mathscr{F}}$, et homotope dans $\mathbf{D} \backslash \widetilde{\mathrm{X}}$ à 
$\Gamma=\prod_{i=0}^{q-1} \gamma_{f^{i}(z)}$. Comme précedemment, on montre qu'il existe deux points $z_{1}$ et $z_{2}$ de $\tilde{\mathrm{X}}$ tels que le nombre de rotation de l'orbite de $z_{0}$ dans $\mathrm{A}_{z_{1}, z_{2}}$ est

$$
\frac{1}{q}\left(\operatorname{Ind}_{\Gamma_{0}}\left(z_{1}\right)-\operatorname{Ind}_{\Gamma_{0}}\left(z_{2}\right)\right) \neq 0
$$

Commençons la démonstration de la proposition 11.3. Nous pouvons toujours supposer, grâce au lemme 11.4, que $\mathrm{X}=\mathrm{Fix}_{*}(\mathrm{~F})$ est non enlacé et que tout point périodique contractile est fixe. On considère une isotopie $\left(\mathrm{F}_{t}^{\prime}\right)_{t \in[0,1]}$ de l'identité à $\mathrm{F}$ qui laisse fixe tout point de $\mathrm{X}$ et un feuilletage $\mathscr{F}$ sur $\mathrm{M} \backslash \mathrm{X}$ transverse à l'isotopie $\left(\mathrm{F}_{t}^{\prime}\right)_{t \in[0,1]}$. Pour tout $k \geq 1$, le feuilletage $\mathscr{F}$ est transverse à l'isotopie obtenue par assemblages de l'isotopie $\left(\mathrm{F}_{t}^{\prime}\right)_{t \in[0,1]}$ et qui joint l'identité à $\mathrm{F}^{k}$. Les seuls points périodiques contractiles de $\mathrm{F}$ sont les points de $\mathrm{X}$, cet ensemble est donc un ensemble non enlacé maximal de $\mathrm{F}^{k}$, pour tout $k \geq 1$ Grâce aux assertions iv) et $\mathbf{v}$ ) de la proposition 10.4 , on en déduit que pour tout point fixe $z \in \mathrm{X}$ et pour tout $k \geq 1$, on a

$$
i\left(\mathrm{~F}^{k}, z\right)=i(\mathscr{F}, z)=i(\mathrm{~F}, z)
$$

Relevons le feuilletage $\mathscr{F}$ en un feuilletage $\widetilde{\mathscr{F}}$ défini sur $\mathbf{D} \backslash \widetilde{\mathrm{X}}$, où $\tilde{\mathrm{X}}=\pi^{-1}(\mathrm{X})$, et notons $\lambda: \widetilde{\mathrm{X}} \rightarrow \mathbf{Z}$ le relèvement de la fonction $\Lambda: \mathrm{X} \rightarrow \mathbf{Z}$ construite dans la preuve de la proposition 10.4. Toute feuille de $\widetilde{\mathscr{F}}$ définit une connexion entre deux points de $\widetilde{\mathrm{X}}$. On appellera suite de connexions entre deux points $z$ et $z^{\prime}$ de $\widetilde{\mathrm{X}}$ une suite $\left(\phi_{j}\right)_{0 \leq j \leq r}$ de connexions, où

$$
\alpha\left(\phi_{0}\right)=z ; \quad \omega\left(\phi_{r}\right)=z^{\prime} ; \quad \omega\left(\phi_{j}\right)=\alpha\left(\phi_{j+1}\right), \quad \text { si } 0 \leq j<r .
$$

Nous utiliserons le lemme suivant :

Lemme 11.5. - La proposition 11.3 est vérifiée dans le cas suivant : il existe une partie $\mathrm{K} \subset \mathbf{D}$ invariante par $f$, compacte, connexe et pleine (i.e. de complémentaire connexe) qui contient deux points $z_{0}$ et $z_{1}$ de $\tilde{\mathrm{X}}$ qui sont liés par une suite de connexions. Plus précisément, $\mathrm{K}$ est alors d'intérieur non vide et contient des points $z$ distincts de $z_{0}$ et $z_{1}$ ayant un vecteur de rotation non nul dans $\mathrm{A}_{z_{0}, z_{1}}$ pour $\widehat{f}_{z_{0}, z_{1}}$.

Démonstration. - Notons $\pi: \widehat{\mathrm{A}}_{z_{0}, z_{1}} \rightarrow \mathrm{A}_{z_{0}, z_{1}}$ le revêtement universel de $\mathrm{A}_{z_{0}, z_{1}}$. Considérons une suite de connexions $\left(\phi_{j}\right)_{0 \leq j \leq r}$, où

$$
\alpha\left(\phi_{0}\right)=z_{0} ; \quad \omega\left(\phi_{r}\right)=z_{1} ; \omega\left(\phi_{j}\right)=\alpha\left(\phi_{j+1}\right), \quad \text { si } 0 \leq j<r,
$$


et posons $\Gamma=\prod_{j=0}^{r} \phi_{j}$. Considérons alors une composante connexe $\widehat{\Gamma}$ de $\widehat{\pi}^{-1}(\Gamma)$. Remarquons que $\widehat{\Gamma}$ sépare $\widehat{\mathrm{A}}_{z_{0}, z_{1}}$ en deux ouverts, l'un $\mathrm{W}_{d}$ situé à droite et l'autre $\mathrm{W}_{g}$ situé à gauche. Remarquons également que

$$
\widehat{f}_{z 0, z 1}\left(\overline{\mathrm{W}}_{d}\right) \subset \mathrm{W}_{d} \cup\left\{z^{1}, \ldots, z^{r}\right\}, \quad \widehat{f}_{z 0, z 1}^{-1}\left(\overline{\mathrm{W}}_{g}\right) \subset \mathrm{W}_{g} \cup\left\{z^{1}, \ldots, z^{r}\right\},
$$

où $z^{j}$ est l'antécédent par $\widehat{\pi}$ de $\alpha\left(\phi_{j}\right)$ situé sur $\widehat{\Gamma}$.

En particulier les ensembles

$$
\mathrm{W}_{g} \cap \widehat{f}_{z_{0}, z_{1}}^{-1}\left(\mathrm{~W}_{d}\right), \quad \widehat{f}_{z_{0}, z_{1}}\left(\mathrm{~W}_{g}\right) \cap \mathrm{W}_{d}
$$

sont des ouverts non vides errants. Nous allons commencer par montrer que ces ensembles sont inclus dans $\widehat{\pi}^{-1}\left(\mathrm{~K} \backslash\left\{z_{0}, z_{1}\right\}\right)$, ce qui prouvera que $\mathrm{K}$ est d'intérieur non vide. Supposons le contraire. L'ensemble

$$
\mathrm{U}=(\mathbf{D} \backslash \mathrm{K}) \sqcup\{\infty\}
$$

est un disque ouvert de $\mathrm{A}_{z_{0}, z_{1}}$ et $\widehat{\pi}^{-1}(\mathrm{U})$ est réunion disjointe de disques ouverts homéomorphes par $\widehat{\pi}$ à U. Chacun d'eux contient un antécédent de $\infty$. Comme cet antécédent est un point fixe de $\widehat{f}_{z_{0}, z_{1}}$, chacun des disques est invariant. On fixe une de ces composantes, notée $\widehat{\mathrm{U}}$, qui rencontre $\mathrm{W}_{g} \cap \widehat{f}_{z_{0}, z_{1}}^{-1}\left(\mathrm{~W}_{d}\right)$ et on note $\widehat{\infty}$ l'antécédent de $\infty$ qu'elle contient. L'une des deux parties $\widehat{\mathrm{U}} \cap \mathrm{W}_{d}$ et $\widehat{\mathrm{U}} \cap \mathrm{W}_{g}$ ne contient pas $\widehat{\infty}$ et est donc de mesure finie (pour la mesure $\widehat{\mu}$ qui relève $\mu$ ). Elle est positivement ou négativement invariante par $\widehat{f}_{z_{0}, z_{1}}$ et donc formée de points non errants. Nous avons obtenu une contradiction.

En utilisant le fait que $\mathrm{K}$ est de mesure finie non nulle, contrairement à $\mathbf{D}$, et par des arguments similaires à ceux employés dans la démonstration du théorème 10.1 , on montre alors que presque tout point de $\widehat{W}_{g} \cap f_{z_{0}, z 1}^{-1}\left(\mathrm{~W}_{d}\right)$ a un vecteur de rotation non nul (éventuellement infini).

En étudiant la dynamique de $\mathscr{F}$, nous avons vu qu'il existait au moins un puits et une source. Fixons donc un puits $z_{0} \in \tilde{\mathrm{X}}$, puis considérons l'ensemble W, réunion de $z_{0}$ et des feuilles $\phi \in \widetilde{\mathscr{F}}$ telles que $\omega(z)=z_{0}$. Il s'agit d'un disque ouvert. Nous allons étudier la frontière de W. Commençons par montrer le résultat simple suivant :

Lemme 11.6. - La partie $\mathrm{W}$ est bornée et sa frontière est réunion de feuilles et de singularités z qui peuvent être jointes à zo par une suite de connexions.

Démonstration. - Rappellons qu'il existe un lacet $\Gamma$ dans $\mathrm{M}$ homologue à zéro et une fonction $\Lambda$ associée à $\Gamma$ définie sur $M \backslash \Gamma$ qui décroit le long des feuilles 
et que l'adhérence de toute composante connexe $\mathrm{U}$ de $\mathrm{M} \backslash \Gamma$ est un disque fermé. L'ensemble $\widehat{\pi}^{-1}(\mathrm{U})$ est formé également de disques fermés. On obtient ainsi un recouvrement de $\mathbf{D}$ par une famille $\left(\Delta_{i}\right)_{i \in \mathrm{I}}$ de disques fermés. Toute feuille $\phi \subset \mathrm{W}$ rencontre un nombre fini, au plus égal à $\max \Lambda-\min \Lambda$, de disques $\Delta_{i}$, ainsi $\mathrm{W}$ est relativement compact.

Il est clair que si $z \in \partial \mathrm{W}$ n'appartient pas à $\tilde{\mathrm{X}}$, la feuille passant par $z$ est incluse dans $\partial \mathrm{W}$. Un point $z \in \widetilde{\mathrm{X}} \cap \partial \mathrm{W}$ est une source ou une selle. Si c'est une source, il y a une connexion entre $z$ et $z_{0}$. Si c'est une selle, il y a une feuille $\phi$ issue de $z$ qui est contenue dans $\bar{W}$ puisque $z \in \partial \mathrm{W}$. Pour montrer qu'il y a une suite de connexions entre $z$ et $z_{0}$, on raisonne par l'absurde. Le point $\omega(\phi)$ est alors différent de $z_{0}$ et il n'y a pas de connexion entre ce point et $z_{0}$. En particulier c'est un point selle et on peut recommencer le processus. On obtient une suite infinie $\left(z^{i}\right)_{i \geq 0}$ de points de $\widetilde{\mathrm{X}}$ avec une connexion entre $z^{i}$ et $z^{i+1}$, en particulier on sait que la suite $\left(\lambda\left(z^{i}\right)\right)_{i \geq 0}$ est strictement décroissante. Ceci est impossible puisque la fonction $\lambda$ prend un nombre fini de valeurs sur $\tilde{\mathrm{X}}$.

On peut écrire

$$
\mathrm{W}=\bigcup_{n \geq 0} \mathrm{D}_{n},
$$

où $\left(\mathrm{D}_{n}\right)_{n \geq 0}$ est une suite croissante de disques fermés dont l'intérieur contient $z_{0}$. On note alors $\mathrm{K}_{n}$ la composante connexe de $\bigcap_{k \in \mathbf{Z}} f^{-k}\left(\mathrm{D}_{n}\right)$ qui contient $z_{0}$. C'est une partie compacte connexe pleine, invariante $\operatorname{par} f$.

Lemme 11.7. - L'ensemble $\mathrm{K}_{n}$ rencontre $\partial \mathrm{D}_{n}$.

Démonstration. - On raisonne par l'absurde en supposant que $\mathrm{K}_{n} \cap \partial \mathrm{D}_{n}=\emptyset$. Si $\check{\mathbf{D}}$ est le plan topologique obtenu à partir de $\mathbf{D}$ en identifiant $\mathbf{K}_{n}$ à un point et si $\check{f}$ est l'homéomorphisme de $\check{\mathbf{D}}$ fixant le point $\mathbf{K}_{n}$, alors pour tout entier $k \geq 1$, on a

$$
i\left(\check{f}^{k}, \mathrm{~K}_{n}\right)=i\left(f^{k}, z\right)=1 .
$$

Puisque $\mathrm{K}_{n} \cap \partial \mathrm{D}_{n}=\emptyset$, on peut trouver un disque fermé $\mathrm{D}^{\prime} \subset \mathrm{D}_{n}$ dont la frontière est disjointe de l'ensemble $\bigcap_{k \in \mathbf{Z}} f^{-k}\left(\mathrm{D}_{n}\right)$, et dont l'intérieur contient $\mathrm{K}_{n}$. Le disque $\check{\mathrm{D}}^{\prime}$, image naturelle de $\mathrm{D}^{\prime}$ dans $\check{\mathbf{D}}$ est alors un bloc isolant de $\check{f}$, c'est-à-dire que

$$
\bigcap_{k \in \mathbf{Z}} \check{f}^{-k}\left(\check{\mathrm{D}}^{\prime}\right) \cap \partial \check{\mathrm{D}}^{\prime}=\emptyset .
$$

On sait d'autre part que la seule orbite périodique incluse dans $\check{\mathrm{D}}^{\prime}$ est le point fixe $\mathrm{K}_{n}$. On sait alors, d'après [Fr5] ou [LY], qu'il existe un entier $k \geq 1$ tel que l'indice de Lefschetz $i\left(\breve{f}^{k}, \mathrm{~K}_{n}\right)$ est négatif ou nul, ce qui contredit l'hypothèse. 
La suite $\left(\mathrm{K}_{n}\right)_{n \geq 0}$ est croissante et l'ensemble $\mathrm{K}=\overline{\bigcup_{n \geq 0} \mathrm{~K}_{n}}$ est une partie compacte connexe invariante par $f$, contenant $z_{0}$ et rencontrant $\partial \mathrm{W}$. On va démontrer que $\mathrm{K}$ contient un point $z \in \widetilde{\mathrm{X}} \cap \partial \mathrm{W}$. En utilisant le lemme 11.6, puis en appliquant le lemme 11.5 au complémentaire de la composante connexe non relativement compacte (dans $\mathbf{D}$ ) de $\mathrm{K}$, on obtiendra la proposition 11.2. Il reste donc à montrer :

\section{Lemme 11.8. - L'ensemble $\mathrm{K}$ contient une singularité z.}

Démonstration. - Le raisonnement est du même type que celui du lemme 11.4. Soit $z_{0}^{\prime} \in \mathrm{K} \cap \partial \mathrm{W}$. Si $z_{0}^{\prime}$ n'est pas une singularité, on considère la feuille $\phi$ de $\tilde{\mathscr{F}}$ passant pas $z_{0}^{\prime}$ ainsi que les points $z_{1}=\alpha(\phi)$ et $z_{2}=\omega(\phi)$. Notons $\widehat{\pi}: \widehat{\mathrm{A}}_{z_{1}, z_{2}} \rightarrow \mathrm{A}_{z_{1}, z_{2}}$ le revêtement universel de $\mathrm{A}_{z_{1}, z_{2}}$ et fixons une composante connexe $\widehat{\phi}$ de $\pi^{-1}(\phi)$. Elle sépare $\widehat{\mathrm{A}}_{z_{1}, z_{2}}$ en deux ouverts, l'un $\mathrm{W}_{d}$ situé à droite et l'autre $\mathrm{W}_{g}$ situé à gauche, qui vérifient

$$
\widehat{f}_{z_{1}, z_{2}}\left(\overline{\mathrm{W}}_{d}\right) \subset \mathrm{W}_{d}, \quad \widehat{f}_{z_{1}, z_{2}}^{-1}\left(\overline{\mathrm{W}}_{g}\right) \subset \mathrm{W}_{g} .
$$

Notons $\widehat{z}_{0}^{\prime}$ l'antécédent par $\widehat{\pi}$ de $z_{0}^{\prime}$ situé sur $\widehat{\phi}$. L'ensemble

$$
\widehat{f}_{z_{1}, z_{2}}^{-1}\left(\mathrm{~W}_{d}\right) \cap \widehat{f}_{z_{1}, z_{2}}\left(\mathrm{~W}_{g}\right)
$$

est un voisinage de $\widehat{z}_{0}^{\prime}$ et rencontre donc une certaine composante connexe $\widehat{\mathrm{K}}_{n}$ de $\widehat{\pi}^{-1}\left(\mathrm{~K}_{n}\right)$, si $n$ est assez grand. Cette composante est fixe et homéomorphe, par $\widehat{\pi}$, à $\mathrm{K}_{n}$, puisque $\mathrm{K}_{n}$ est une partie compacte connexe pleine disjointe de $\bar{\phi}$. La partie $\widehat{\mathrm{K}}_{n}$ doit donc rencontrer $\mathrm{W}_{g} \cap \widehat{f}_{z_{1}, z_{2}}^{-1}\left(\mathrm{~W}_{d}\right)$ ou $\widehat{f}_{z_{1}, z_{2}} \mathrm{~W}_{g} \cap\left(\mathrm{W}_{d}\right)$. Elle doit en fait rencontrer ces deux ensembles puisque l'un d'eux est l'image de l'autre. Ceci est impossible puisque $\widehat{\mathrm{K}}_{n} \cap \widehat{\phi}=\emptyset$.

\section{BIBLIOGRAPHIE}

[Bi] G. D. Birkhoff, An extension of Poincaré's last geometric theorem, Acta Math., 47 (1925), $297-311$.

[Br] L. E. J. Brouwer, Beweis des ebenen Translationssatzes, Math. Ann., 72 (1912), 37-54.

[Bn] M. Brown, A new proof of Brouwer's lemma on translation arcs, Houston 7. Math., 10 (1984), 35-41.

[BST] M. Brown, E. Slaminka, and W. Transue, An orientation preserving fixed point free homeomorphism of the plane which admits no closed invariant line, Topol. Appl., 29 (1988), 213-217.

[Ca] P. H. Carter, An improvment of the Poincaré-Birkhoff theorem, Trans. Am. Math. Soc., 269 (1982), $285-299$.

[Co] G. Conley, Isolated invariants sets and Morse index, CBMS Reg. Conf. Series in Math., Am. Math. Soc., 38 (1978).

[Fa] A. Fathi, An orbit closing proof of Brouwer's lemma on translation arcs, Enseign. Math., 33 (1987), 315-322.

[Flo] A. FlokR, Proof of the Arnold conjectures for surfaces and generalizations to certain Kähler manifolds, Duke Math. F., 51 (1986), 1-32.

[Flu] M. Flucher, Fixed points of measure preserving torus homeomorphism, Manuscr. Math., 68 (1990), $271-293$. 
[Frl] J. Franks, Generalizations of the Poincaré-Birkhoff theorem, Ann. Math., 128 (1988), 139-151.

[Fr2] J. Franks, Recurrence and fixed points of surface homeomorphisms, Ergodic Theory Dyn. Syst., 8* (1988), 99-107.

[Fr3] J. Franks, A new proof of the Brouwer plane translation theorem, Ergodic Theory Dyn. Syst., 12 (1992), 217-226.

[Fr4] J. Franks, Geodesics on $\mathrm{S}^{2}$ and periodic points of annulus homeomorphisms, Invent. Math., 108 (1992), 403-418.

[Fr5] J. Franks, Area preserving homeomorphisms of open surfaces of genus zero, New York f. Math., 2 (1996), $1-19$.

[Fr6] J. Franks, The Conley index and non-existence of minimal homeomorphisms, Ill. F. Maths, 43 (1999), 457-464.

[FH] J. Franks and M. Handel, Periodic points of Hamiltonian surface diffeomorphims, Geom. Topol., 7 (2003), $713-756$.

[GL] J.-M. Gambaudo et P. Le Calvez, Infinité d'orbites périodiques pour les difféomorphismes conservatifs de l'anneau, Mém. Soc. Math Fr., 79 (1999), 125-146.

[Gu L. Guillou, Théorème de translation plane de Brouwer et généralisations du théorème de Poincaré-Birkhoff, Topology, 33 (1994), 331-351.

[Haml] M.-E. Hamstrom, The space of homeomorphisms on a torus, Ill. f. Math., 9 (1965), 59-65.

[Ham2] M.-E. Hamstrom, Homotopy groups of the space of homeomorphisms on a 2-manifold, Ill. f. Math., 10 (1966), 563-573.

[Han1] M. Handel, There are no minimal homeomorphisms of the multipunctured plane, Ergodic Theory Dyn. Syst., 12 (1992), 75-83.

[Han2] M. Handel, A fixed point theorem for planar homeomorphisms, Topology, 38 (1999), 235-264.

[HT] M. Handel and W. P. Thurston, New proofs of some results of Nielsen, Adv. Math., 56 (1985), 173191

[HZ] H. Hofer and E. Zehnder, Symplectic invariants and Hamiltonian dynamics, Birkhäuser, Basel-Boston-Berlin (1994).

[K] B. DE KERÉKJÁRTó, The plane translation theorem of Brouwer and the last geometric theorem of Poincaré, Acta Sci. Math. Szeged, 4 (1928-29), 86-102.

[J] B. Jiang, Lectures on Nielsen Fixed Point Theory, Contemp. Math., 14 (1983).

[LeC1] P. Le Calvez, Propriétés dynamiques de l'anneau et du tore, Astérisque, 204 (1991).

[LeC2] P. Le Calvez, Une généralisation du théorème de Conley-Zehnder aux homéomorphismes du tore de dimension deux, Ergodic Theory Dyn. Syst., 17 (1997), 71-86.

[LeC3] P. Le Calvez, Une propriété dynamique des homéomorphismes du plan au voisinage d'un point fixe d'indice > 1, Topology, 38 (1999), 25-35.

[LeC4] P. Le Calvez, Dynamique des homéomorphismes du plan au voisinage d'un point fixe, Ann. Sci. Éc. Norm. Supér., IV Sér., 36 (2003), 139-171.

[LeC5] P. Le Galvez, Une version feuilletée du théorème de translation de Brouwer, Comment. Math. Helv., 21 (2004), 229-259.

[LS] P. Le Calvez et A. Sauzet, Une démonstration dynamique du théorème de translation de Brouwer, Expo. Math., 14 (1996), 277-287.

[LY] P. Le Galvez et J.-G. Yoccoz, Suite des indices de Lefschetz des itérés pour un domaine de Jordan qui est un bloc isolant. Manuscrit.

[LeR] F. LE Roux, Homéomorphismes de surfaces, théorème de la fleur de Leau-Fatou et de la variété stable, Astérisque, 292 (2004).

[M] S. Matsumoto, Arnold conjecture for surface homeomorphisms, Proceedings of the French-Japanese Conference "Hyperspace Topologies and Applications" (La Bussière, 1997), Topol. Appl., 104 (2000), 191-214.

[N] N. A. Nikishin, Fixed points of diffeomorphisms of two-dimensional spheres preserving an oriented plane, Funkts. Anal. Prilozh., 8 (1974), 84-85.

[PS] S. Pelikan and E. Slaminka, A bound for the fixed point index of area-preserving homeomorphisms of two-manifolds, Ergodic Theory Dyn. Syst., 7 (1987), 463-479.

[SZ] D. SAlamon and E. Zehnder, Morse theory for periodic solutions of Hamiltonian systems and the Maslov index, Commun. Pure Appl. Math., 45 (1992), 1303-1360. 
[Sa] A. Sauzet, Application des décompositions libres à l'étude des homéomorphismes de surface, Thèse de l'Université Paris 13 (2001).

[Scz] M. Schwarz, On the action spectrum for closed symplectically aspherical manifolds, Pac. F. Math., 193 (2000), 419-461.

[Scn] S. Schwartzman, Asymptotic cycles, Ann. Math., 68 (1957), 270-284.

[Sik] J.-C. Sikorav, Points fixes d'une application symplectique homologue à l'identité, f. Differ. Geom., 22 (1985), 49-79.

[Sim] C. P. Simon, A bound for the fixed-point index of an area-preserving map with applications to mechanics, Invent. Math., 26 (1974), 187-200.

P. L. C.

Laboratoire Analyse, Géométrie et Applications,

G.N.R.S.-U.M.R 7539, Institut Galilée,

Université Paris 13,

Avenue J.-B. Clément,

93430 Villetaneuse, France

lecalvez@math.univ-paris13.fr

Manuscrit reçu le 4 mars 2004. 